JOURNAL OF

SYMPLECTIC GEOMETRY

Volume 2, Number 4, 445-543, 2005

\title{
ENUMERATIVE VS. SYMPLECTIC INVARIANTS AND OBSTRUCTION BUNDLES
}

AleKSey Zinger

We describe in detail a gluing construction for pseudoholomorphic maps in symplectic geometry, including in the presence of an obstruction bundle. The main motivation is to try to compare the symplectic and enumerative invariants of algebraic manifolds. These descriptions can also be used to enumerate rational curves with high-order degeneracies of local nature in projective spaces.

\section{Contents}

1. Introduction 446

1.1. Background and Motivation 446

1.2. Summary 448

1.3. Fundamental Notation $\quad 450$

2. Spaces of Bubble Maps 453

2.1. Bubble Trees 453

2.2. The Basic Gluing Construction 455

2.3. Curves with Marked Points 459

2.4. Bubble Maps 461

2.5. Strata of Bubble Maps 464

3. The Gluing Construction and the Obstruction Bundle 468

3.1. Summary and Notation 468

3.2. The Basic Setup 471

3.3. Construction of Nearly Holomorphic Bubble Maps 476

3.4. Scale of Variations 479

3.5. Obstruction Bundle Setup 484

3.6. The Gluing Map 489 
3.7. An Implicit Function Theorem

3.8. The Orientation of $\mathcal{M}_{\Sigma, t \nu, \lambda}(\mu)$ and the Gluing Map 494

3.9. Gluing Maps for Spaces $\overline{\mathcal{U}}_{\mathcal{T}}^{(0)}(\mu)$ and Orientations 504

4. Technical Issues 508

4.1. Continuity of the Gluing Map 508

4.2. Injectivity of the Gluing Map 513

4.3. The Basic Gluing Map and the Space of Balanced Maps 516

4.4. Gromov Convergence and the $L^{p}$-norm of the Differential 522

4.5. Surjectivity of the Gluing Map 527

5. Appendix 530

5.1. Properties of Smooth Families of Metrics on $\Sigma \quad 530$

5.2. Sobolev Inequalities for the Metrics $g_{v} \quad 534$

5.3. Elliptic Estimates for the Metrics $g_{v} \quad 535$

5.4. Fiber-Uniform Inverse for the Operator $D_{v} \quad 540$

$\begin{array}{ll}\text { References } & 542\end{array}$

\section{Introduction}

1.1. Background and Motivation. Suppose $(\Sigma, j)$ is a nonsingular Riemann surface of genus $g \geq 2$ and $(V, J, \omega)$ is a Kahler manifold of complex dimension $n$. If $\lambda \in H_{2}(V ; \mathbb{Z})$, denote by $\mathcal{H}_{\Sigma, \lambda}(V)$ the set of simple $(J, j)$ holomorphic maps $u$ from $\Sigma$ to $V$ such that $u_{*}[\Sigma]=\lambda$. Let $\mu=\left(\mu_{1}, \ldots, \mu_{N}\right)$ be an $N$-tuple of proper oriented submanifolds of $V$ such that

$$
\operatorname{codim} \mu \equiv \sum_{l=1}^{l=N} \operatorname{codim} \mu_{l}=2\left(\left\langle c_{1}(V, J), \lambda\right\rangle-n(g-1)+N\right) .
$$

For many Kahler manifolds $(V, J, \omega)$ and choice of constraints $\mu$, the cardinality of the set

$$
\begin{aligned}
\mathcal{H}_{\Sigma, \lambda}(\mu) \equiv\left\{\left(\Sigma ; y_{1}, \ldots, y_{N} ; u\right): u \in \mathcal{H}_{\Sigma, \lambda}(V)\right. & \\
& \left.y_{l} \in \Sigma, u\left(y_{l}\right) \in \mu_{l} \forall l=1, \ldots, N\right\}
\end{aligned}
$$

is finite and depends only on the homology classes of $\mu_{1}, \ldots, \mu_{N}$. The cardinality $\left|\mathcal{H}_{\Sigma, \lambda}(\mu)\right|$ of the set $\mathcal{H}_{\Sigma, \lambda}(\mu)$ is then an enumerative invariant of the complex manifold $(V, J)$. Such numbers for algebraic manifolds $(V, J)$, e.g., the complex projective spaces $\mathbb{P}^{n}$, have been of great interest in algebraic geometry for a long time.

If $(V, \omega, J)$ is a semipositive symplectic manifold, the symplectic invariant of $(V, \omega)$,

$$
\mathrm{RT}_{g, \lambda}(; \mu) \equiv \mathrm{RT}_{g, \lambda}\left(; \mu_{1}, \ldots, \mu_{N}\right)
$$


of $[\mathbf{R T}]$, is a well-defined integer. Due to the two composition laws of $[\mathbf{R T}]$, this symplectic invariant is often more readily computable than the enumerative invariant $\left|\mathcal{H}_{\Sigma, \lambda}(\mu)\right|$. In fact, all such symplectic invariants of $\mathbb{P}^{n}$ are easily computable. It is also shown in Section 10 of $[\mathbf{R T}]$ that the appropriately defined genus-zero enumerative invariants of $\mathbb{P}^{n}$ agree with the corresponding symplectic invariants. On the other hand, even for $\mathbb{P}^{2}$ and for genus one, the two invariants are no longer equal. In $[\mathbf{I}]$, the difference

$$
\operatorname{RT}_{1, \lambda}\left(\mu_{1} ; \mu_{2}, \ldots, \mu_{N}\right)-\left|\mathcal{H}_{\Sigma, \lambda}(\mu)\right|
$$

is computed for genus-one surfaces $\Sigma$ and all projective spaces using an obstruction-bundle approach, first introduced by $[\mathbf{T}]$ in a very different setting. In $[\mathbf{Z 2}]$, the difference

$$
\mathrm{RT}_{2, \lambda}(; \mu)-\left|\mathcal{H}_{\Sigma, \lambda}(\mu)\right|
$$

is computed for genus-two surfaces $\Sigma$ for $\mathbb{P}^{2}$ and $\mathbb{P}^{3}$ using a similar approach. Both differences are linear combinations of genus-zero enumerative invariants.

The purpose of this paper is to describe in detail a gluing construction for pseudoholomorphic maps which is suitable for analyzing relationships between symplectic and enumerative invariants of Kahler, or more generally almost Kahler, manifolds. In particular, this paper supplies the most technical portion of the justification needed for the main analytic setups in $[\mathbf{I}]$ and $[\mathbf{Z 2}]$. The explicit nature of the gluing construction can yield useful estimates for obstructions to smoothing pseudoholomorphic maps from singular domains and for the behavior of derivatives of pseudoholomorphic maps under gluing; see Subsection 4.1 and Theorem 2.8 in [Z2]. Such estimates are used in an essential way in $[\mathbf{I}]$ and $[\mathbf{Z 2}]$.

The power series expansions of Theorem 2.8 and Proposition 4.4 in [Z2], and their analogues in other genera, are useful in both enumerative geometry and Gromov-Witten theory. For example, Theorem 2.8 of [Z2] is used in $[\mathbf{Z 3}]$ to describe a method for solving a large class of enumerative problems involving rational curves in $\mathbb{P}^{n}$. On the other hand, a genus-one analogue of Proposition 4.4 in $[\mathbf{Z 2}]$ is used in $[\mathbf{Z 4}]$ to describe the "main component" $\overline{\mathcal{M}}_{1, k}^{0}(V, \lambda)$ of the moduli space $\overline{\mathcal{M}}_{1, k}(V, \lambda)$ of genus-one stable maps into $V$. This main component is a closed subset of $\overline{\mathcal{M}}_{1, k}(V, \lambda)$ and contains the subspace $\mathcal{M}_{1, k}^{0}(V, \lambda)$ of $\overline{\mathcal{M}}_{1, k}(V, \lambda)$ consisting of stable $J$-holomorphic maps with smooth domains. If $J$ is sufficiently regular, $\overline{\mathcal{M}}_{1, k}^{0}(V, \lambda)$ is the closure of $\mathcal{M}_{1, k}^{0}(V, \lambda)$ and carries a fundamental class.

The author is grateful to T. Mrowka for pointing out the paper $[\mathbf{I}]$, encouraging the author to work out all of the analytic issues arising in $[\mathbf{I}]$, and sharing some of his expertise in applications of global analysis over countless 
hours of conversations. The author would also like to thank G. Tian, for first introducing him to Gromov's symplectic invariants and helping him understand $[\mathbf{L T}]$, and the referee, for corrections and suggestions on the original version of this paper.

1.2. Summary. In this subsection, we first recall the definition of GromovWitten fixed-complex structure invariants for a semi-positive almost Kahler manifold $(V, J, \omega)$. We then outline the rest of the paper and roughly describe the statements of the two main theorems.

If $\Sigma$ and $V$ are as in the previous subsection, we denote by

$$
\pi_{\Sigma}, \pi_{V}: \Sigma \times V \longrightarrow \Sigma, V
$$

the two projection maps. Let

$$
\Lambda^{0,1} \pi_{\Sigma}^{*} T^{*} \Sigma \otimes \pi_{V}^{*} T V \longrightarrow \Sigma \times V
$$

be the bundle of $(J, j)$-antilinear homomorphisms from $\pi_{\Sigma}^{*} T \Sigma$ to $\pi_{V}^{*} T V$. If

$$
\nu \in \Gamma\left(\Sigma \times V ; \Lambda^{0,1} \pi_{\Sigma}^{*} T^{*} \Sigma \otimes \pi_{V}^{*} T V\right),
$$

we denote by $\mathcal{M}_{\Sigma, \nu, \lambda}$ the set of all smooth maps $u$ from $\Sigma$ to $\mathbb{P}^{n}$ such that

$$
u_{*}[\Sigma]=\lambda \quad \text { and }\left.\quad \bar{\partial} u\right|_{z}=\left.\nu\right|_{(z, u(z))} \quad \forall z \in \Sigma .
$$

If $\mu$ is an $N$-tuple of constraints as above, put

$$
\begin{aligned}
\mathcal{M}_{\Sigma, \nu, \lambda}(\mu)=\left\{\left(\Sigma ; y_{1}, \ldots, y_{N} ; u\right):\right. & u \in \mathcal{M}_{\Sigma, \nu, \lambda} ; \\
y_{l} & \left.\in \Sigma, u\left(y_{l}\right) \in \mu_{l} \forall l=1, \ldots, N\right\} .
\end{aligned}
$$

If $(V, \omega, J)$ is semipositive, for generic choices of $\nu$ and $\mu, \mathcal{M}_{\Sigma, \nu, \lambda}$ is a smooth finite-dimensional oriented manifold, and $\mathcal{M}_{\Sigma, \nu, \lambda}(\mu)$ is a zero-dimensional finite submanifold of $\mathcal{M}_{\Sigma, \nu, \lambda} \times \Sigma^{N}$, whose signed cardinality depends only the homology classes of $\mu_{1}, \ldots, \mu_{N}$; see Section 1 of $[\mathbf{R T}]$. The symplectic invariant $\mathrm{RT}_{g, \lambda}(; \mu)$ is the signed cardinality of the set $\mathcal{M}_{\Sigma, \nu, \lambda}(\mu)$.

If $\left\|\nu_{i}\right\|_{C^{0}} \longrightarrow 0$ and $\left(\Sigma ; \underline{y}_{i} ; u_{i}\right) \in \mathcal{M}_{\Sigma, \nu_{i}, \lambda}(\mu)$, a subsequence of $\left\{\left(\Sigma ; \underline{y}_{i}, u_{i}\right)\right\}_{i=1}^{\infty}$ must converge in the Gromov topology to one of the following:

(1) an element of $\mathcal{H}_{\Sigma, \lambda}(\mu)$;

(2) $\left(\Sigma_{\top} ; \underline{y} ; u\right)$, where $\Sigma_{\top}$ is a bubble tree of $S^{2}$ 's attached to $\Sigma$ with marked points $y_{1}, \ldots, y_{N}$, and $u: \Sigma_{\top} \longrightarrow V$ is a holomorphic map such that $u\left(y_{l}\right) \in \mu_{l}$ for $l=1, \ldots, N$, and

(2a) $\left.u\right|_{\Sigma}$ is simple and the tree contains at least one $S^{2}$;

(2b) $\left.u\right|_{\Sigma}$ is multiply-covered;

(2c) $\left.u\right|_{\Sigma}$ is constant and the tree contains at least one $S^{2}$.

This convergence statement says that for all $t$ sufficiently small, every element of $\mathcal{M}_{\Sigma, t \nu, \lambda}(\mu)$ lies near one of the spaces described by (1)-(2c). In many practical applications it is easy to show that there is a bijection between the elements of $\mathcal{H}_{\Sigma, \lambda}(\mu)$ and the nearby elements of $\mathcal{M}_{\Sigma, t \nu, \lambda}(\mu)$; see Proposition 3.30. This is the case for all projective spaces, provided $\lambda$ is a 
sufficiently high multiple of the line. In $[\mathbf{I}]$ and $[\mathbf{Z 2}]$, Cases (2a) and (2b) do not occur, but they may have to be considered when dealing with higherdimensional projective spaces or higher genera. If the signed cardinality of $\mathcal{M}_{\Sigma, t \nu, \lambda}(\mu)$ is $\operatorname{RT}_{g, \lambda}(; \mu)$ for all $t>0$ sufficiently small, the number of elements of $\mathcal{M}_{\Sigma, t \nu, \lambda}(\mu)$ that lie near the spaces described by (2) is thus exactly

$$
C R_{g, \lambda}(\mu) \equiv \operatorname{RT}_{g, \lambda}(; \mu)-\left|\mathcal{H}_{\Sigma, \lambda}(\mu)\right| \text {. }
$$

The goal of this paper is to describe $C R_{g, \lambda}(\mu)$ in terms of the spaces of holomorphic maps themselves, which can be viewed as an enumerative object, rather than a symplectic one. We do need to assume that certain spaces of holomorphic maps are smooth, but they do not need to have the expected dimension.

While there is a very good understanding of what constitutes a stable map, there is little in a way of commonly accepted notation for stable maps and various spaces of stable maps. In Section 2, we recall the definition of bubble or stable maps as well as set up analytically convenient notation. Our notation for bubble maps evolved from that of D. McDuff's lectures at Harvard. In Subsection 2.4, we restate the definition of the Gromov topology on the set of all bubble maps in our notation. In Subsection 2.5, we define various spaces $\mathcal{M}_{\mathcal{T}}$ and $\mathcal{U}_{\mathcal{T}}$ of bubble maps and bundles of gluing parameters $F \mathcal{T}$ over $\mathcal{M}_{\mathcal{T}}$ and $\mathcal{F} T$ over $\mathcal{U}_{\mathcal{T}}$.

As is typical in symplectic geometry, our gluing construction has two steps: pregluing and perturbation. The pregluing step is usually carried out in the target space $V$. In this paper, we work with the domains to construct an approximately holomorphic map. Indeed, given a pseudoholomorphic map $b=\left(\Sigma_{b}, u_{b}\right)$ in $\mathcal{M}_{\mathcal{T}}\left(\right.$ or $\left.\mathcal{U}_{\mathcal{T}}\right)$ and a gluing parameter $v \in F_{b} \mathcal{T}\left(\right.$ or $\left.v \in \mathcal{F}_{b} \mathcal{T}\right)$ for $b$, we construct a Riemann surface $\Sigma_{v}$ and a nearly holomorphic map

$$
q_{v}: \Sigma_{v} \longrightarrow \Sigma_{b}
$$

see Subsection 2.2. We then take the approximately holomorphic map corresponding to $v$ to be

$$
b(v)=\left(\Sigma_{v}, u_{v} \equiv u_{b} \circ q_{v}\right) .
$$

This explicit construction at the pregluing step leads to the estimates of Theorem 2.8 and Proposition 4.4 in [Z2].

For the second step of a typical gluing construction, one needs to define a family of spaces $\tilde{\Gamma}_{+}(v)$ of admissible perturbations of $b(v)$ and sometimes a family of obstruction bundles $\Gamma_{-}^{0,1}(v)$, which together will be called an obstruction bundle setup. The former space should be a maximal subspace of all perturbations $\Gamma(v)$ of $b(v)$ on which a certain operator $D_{v}$ is fiberwise uniformly invertible, i.e., the norm of its inverse may depend on $b$, but not on $v \in F_{b} \mathcal{T}$. The obstruction bundle $\Gamma_{-}^{0,1}(v)$ should be the complement of the image of $D_{v}$ on $\tilde{\Gamma}_{+}(v)$ in the target space of $D_{v}$ and should be isomorphic 
to the cokernel $\Gamma_{-}^{0,1}(b)$ of a certain operator $D_{b}$. It may appear there are obvious choices for $\tilde{\Gamma}_{+}(v)$ and $\Gamma_{-}^{0,1}(v)$, i.e., the high eigenspaces of $D_{v}^{*} D_{v}$ and the low eigenspaces of $D_{v} D_{v}^{*}$. These spaces, however, are not an option for an obstruction bundle setup. The usual difficulty with the second step of gluing constructions in symplectic geometry is that the operator $D_{v}^{*} D_{v}$ has eigenvalues that tend to zero as the gluing parameter tends to zero, but then disappear as the gluing parameter hits zero. This is not really dealt with in $[\mathbf{I}]$, but there are now several standard approaches to this problem. We use the modified Sobolev norms of [LT], redefined in Subsection 3.3 in the notation of Section 2, and describe the requirements for an obstruction bundle setup in Subsection 3.5.

The main goal of Section 3 is to describe the number of elements of $\mathcal{M}_{\Sigma, t \nu, \lambda}(\mu)$ lying near the stable maps of type (2) in terms of objects intrinsic to the space of such maps. Given a sufficiently regular stratum $\mathcal{S}(\mu) \subset \mathcal{M}_{\mathcal{T}}$ of stable maps of type (2), Theorem 3.29 describes the number of elements of $\mathcal{M}_{\Sigma, t \nu, \lambda}(\mu)$ lying near $\mathcal{S}(\mu)$ as the number of zeros of a map between two vector bundles over $\mathcal{S}(\mu)$. The target vector bundle is the obstruction, or cokernel, bundle $\Gamma_{-}^{0,1}$. The domain vector bundle is the direct sum of the bundle $F \mathcal{T}$ of gluing parameters with the normal bundle of $\mathcal{S}$ in $\mathcal{M}_{\mathcal{T}}$. In Section 4 of $[\mathbf{Z 2}]$, we use convenient choices of an obstruction bundle setup to approximate all such bundle maps by much simpler polynomial bundle maps. The latter maps involve derivatives of rational maps into $\mathbb{P}^{n}$.

We also give a local description of spaces of stable rational maps into $V$ under certain regularity assumptions, i.e., in the unobstructed cases. By Theorem 3.33, the normal bundle of a stratum $\mathcal{U}_{\mathcal{T}}$ in such a space is $\mathcal{F} \mathcal{T}$. This is still the case if generic constraints $\mu$ are imposed on the stable maps. This is a known fact in symplectic, as well algebraic, geometry. However, the explicit nature of the identification maps that appear in the statement of Theorem 3.33 is used to obtain the estimates of Theorem 2.8 in $[\mathbf{Z 2}]$ for the behavior of derivatives of pseudoholomorphic maps.

Section 4 contains proofs of continuity, injectivity, and surjectivity of the gluing maps. These are usually omitted in the literature, but in the given case one has to choose the obstruction bundle setup carefully to ensure that these properties of the gluing map actually hold. In particular, Section 4 contains what $[\mathbf{L T}]$ may mean by "asymptotic analysis near the nodes," which they omit. The appendix deals with even more technical details of the analysis.

We note that the gluing construction described in this paper deals only with attaching rational bubble components to a smooth principal component. However, it can be generalized to allow singular principle components.

1.3. Fundamental Notation. In this subsection, we collect the most frequently used combinatorial and analytic notation. 


\section{Definition 1.1.}

(1) A finite partially ordered set $I$ is a linearly ordered set if for all $i_{1}, i_{2}, h \in I$ such that $i_{1}, i_{2}<h$, either $i_{1} \leq i_{2}$ or $i_{2} \leq i_{1}$.

(2) A linearly ordered set $I$ is a rooted tree if $I$ has a unique minimal element, i.e., there exists $\hat{0} \in I$ such that $\hat{0} \leq h$ for all $h \in I$.

(3) If $I$ and $I^{\prime}$ are linearly ordered sets, bijection $\phi: I \longrightarrow I^{\prime}$ is an isomorphism of linearly ordered sets if for all $h, i \in I, i<h$ if and only if $\phi(i)<\phi(h)$.

A linearly ordered set can be represented by an oriented graph. In Figure 1 , the dots denote the elements of $I$. The arrows specify the partial ordering of the linearly ordered set $I$. By definition, there is at most one outgoing edge at each vertex. A linearly ordered set $I$ is a rooted tree if and only if its graph is connected. The minimal element, or root, $\hat{0}$ of a rooted tree $I$ is the unique vertex of the graph associated to $I$ that has no outgoing edges.
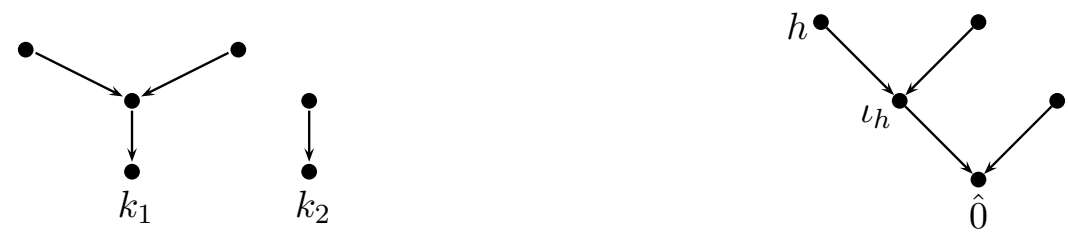

Figure 1. A Linearly Ordered Tree and A Rooted Tree.

If $I$ is a linearly ordered set, we denote the subset of the non-minimal elements of $I$ by $\hat{I}$, i.e.,

$$
\hat{I}=\{h \in I: i<h \text { for some } i \in I\} .
$$

This is the collection of the vertices of the graph corresponding to $I$ that have an outgoing edge. For every $h \in \hat{I}$, the set $\{i \in I: i<h\}$ has a unique maximal element $\iota_{h}$, i.e.,

$$
\iota_{h}<h \quad \text { and } \quad i \leq \iota_{h} \text { for all } i \in I \text { s.t. } i<h .
$$

The vertex $\iota_{h}$ is the endpoint of the unique edge leaving $h$. For reasons clarified in Subsection 2.1, $: \hat{I} \longrightarrow I$ will be called the attaching map of $I$. It is clear from Definition 1.1 that $I$ has a unique splitting

$$
I=\bigsqcup_{k \in K} I_{k}
$$

such that $I_{k} \subset I$ is a rooted tree and $k$ is a minimal element of $I$. The rooted trees $I_{k}$ are the connected components of the graph corresponding to $I$. The 
attaching map of $I$ restricts to the attaching map of each $I_{k}$, which will still be denoted by $\iota$.

Let $I$ be a rooted tree. We denote the unique minimal element of $I$ by $\hat{0}_{I}$, or simply by $\hat{0}$ if there is no ambiguity. If $I^{*}, I_{*}$, and $I *$ are rooted trees, we will write $\hat{I}^{*}, \hat{I}_{*}$, and $\hat{I} *$ for $\widehat{I}^{*}, \widehat{I_{*}}$, and $\widehat{I *}$, respectively; here $*$ denotes any string of symbols. If $i \in I$, let

$$
D_{i} I=\{h \in I: h>i\}, \quad \bar{D}_{i} I=D_{i} I \cup\{i\} .
$$

The subset $D_{i} I$ of $I$ consists of all vertices of $I$ that are "upstream" from $i$. Every rooted tree $I$ has a number of subsets that are rooted trees; the subsets $\bar{D}_{i} I$ are one example. If $H$ is a subset of $I$, the set

$$
I^{(H)} \equiv\{i \in I: i \ngtr h \forall h \in H\}
$$

is also a rooted tree. If $i \in I$, denote $I^{(\{i\})}$ by $I^{(i)}$. If $H$ is a subset of $\hat{I}$, let

$$
I^{H}=\{i \in I: i \nsupseteq h \forall h \in H\}, \quad I(H)=H \cup\left\{\hat{0}_{I}\right\} .
$$

If $h \in \hat{I}$, denote $I^{\{h\}}$ by $I^{h}$.

If $M_{1}$ and $M_{2}$ are two sets, let $M_{1} \sqcup M_{2}$ be the disjoint union of $M_{1}$ and $M_{2}$. Finally, if $N$ is a nonnegative integer, let $[N]=\{1, \ldots, N\}$.

We now introduce some analytic notation. Let $\beta: \mathbb{R} \longrightarrow[0,1]$ be a smooth function such that

$$
\beta(t)=\left\{\begin{array}{ll}
0, & \text { if } t \leq 1 ; \\
1, & \text { if } t \geq 2,
\end{array} \quad \text { and } \quad \beta^{\prime}(t)>0 \text { if } t \in(1,2)\right.
$$

If $r>0$, let $\beta_{r} \in C^{\infty}(\mathbb{R} ; \mathbb{R})$ be given by $\beta_{r}(t)=\beta\left(r^{-\frac{1}{2}} t\right)$. Note that

$$
\operatorname{supp}\left(\beta_{r}\right)=\left[r^{\frac{1}{2}}, 2 r^{\frac{1}{2}}\right], \quad\left\|\beta_{r}^{\prime}\right\|_{C^{0}} \leq C_{\beta} r^{-\frac{1}{2}}, \text { and }\left\|\beta_{r}^{\prime \prime}\right\|_{C^{0}} \leq C_{\beta} r^{-1} .
$$

Throughout the paper, $\beta$ and $\beta_{r}$ will refer to these smooth cutoff functions.

Let $q_{N}, q_{S}: \mathbb{C} \longrightarrow S^{2} \subset \mathbb{R}^{3}$ be the stereographic projections mapping the origin in $\mathbb{C}$ to the north and south poles, respectively. Explicitly,

$$
q_{N}(z)=\left(\frac{2 z}{1+|z|^{2}}, \frac{1-|z|^{2}}{1+|z|^{2}}\right) \in \mathbb{C} \times \mathbb{R}, \quad q_{S}(z)=\left(\frac{2 z}{1+|z|^{2}}, \frac{-1+|z|^{2}}{1+|z|^{2}}\right) .
$$

Denote the south pole of $S^{2}$, i.e., the point $(0,0,-1) \in \mathbb{R}^{3}$, by $\infty$. We identify $\mathbb{C}$ with $S^{2}-\{\infty\}$ via the map $q_{N}$. If $x \in S^{2}-\{\infty\}$, we define the corresponding inverse exponential map

$$
\phi_{x}: S^{2}-\{\infty\} \longrightarrow \mathbb{C} \quad \text { by } \quad \phi_{x} z=z-x \equiv q_{N}^{-1}(z)-q_{N}^{-1}(x) .
$$

Note that this map is a biholomorphism. If $g$ is a Riemannian metric on Riemann surface $(\Sigma, j)$ of positive genus, $x \in \Sigma$ and $v \in T_{x} \Sigma$, we write $\exp _{g, x} v \in \Sigma$ for the exponential of $v$ defined with respect to the Levi-Civita connection of $g$. Let $\operatorname{inj}_{g} x$ denote the corresponding injectivity radius at $x$ 
and $d_{g}$ the distance function. If $x \in \Sigma$, we define the corresponding inverse exponential map

$$
\begin{gathered}
\phi_{g, x}:\left\{z \in \Sigma: d_{g}(x, z)<\operatorname{inj}_{g} x\right\} \longrightarrow T_{x} \Sigma \quad \text { by } \\
\quad \exp _{g, x} \phi_{g, x} z=z, \quad\left|\phi_{g, x} z\right|_{g, x}<\operatorname{inj}_{g} x .
\end{gathered}
$$

Note that if $g$ is flat on a neighborhood $U$ of $x$ in $\Sigma$, then $\left.\phi_{g, x}\right|_{U}$ is holomorphic.

Let $g_{V}$ be the Kahler metric of $(V, J, \omega)$. Denote the corresponding LeviCivita connection, exponential map, and distance function by $\nabla^{V}, \exp _{V}$ and $d_{V}$, respectively. For every $\lambda \in H_{2}(V ; \mathbb{Z})$, let $|\lambda|=\langle\omega, \lambda\rangle$. The number $|\lambda|$ is the $g_{V}$-energy of any element of $\mathcal{H}_{\Sigma, \lambda}$; see Chapter 1 in [MS]. By rescaling $\omega$, it can be assumed that $|\lambda| \geq 1$, whenever $\lambda \neq 0$ and $\mathcal{H}_{S^{2}, \lambda} \neq \emptyset$. If $g$ is any Kahler metric on $(V, J)$, denote the corresponding Levi-Civita connection, exponential map, distance function, injectivity radius, and the parallel transport along the geodesic for $X \in T V$ by $\nabla^{g}, \exp _{g}, d_{g}, \operatorname{inj}_{g}$, and $\Pi_{g, X}$, respectively. If $q \in V$ and $\delta \in \mathbb{R}$, let

$$
B_{g}(q, \delta)=\left\{q^{\prime} \in V: d_{g}\left(q, q^{\prime}\right) \leq \delta\right\} .
$$

In our construction, we allow $g$ vary in a smooth family. Without causing any additional difficulty in the gluing construction, consideration of such families simplifies computations in specific cases such as in $[\mathbf{Z 2}]$. If $(S, j)$ is a smooth Riemann surface and $u \in C^{\infty}(S ; V)$, put

$$
\begin{array}{cl}
\Gamma(u)=\Gamma\left(S ; u^{*} T V\right), & \Gamma^{1}(u)=\Gamma\left(S ; T^{*} S \otimes u^{*} T V\right) \\
\Gamma^{0,1}(u)=\Gamma\left(S ; \Lambda^{0,1} T^{*} S \otimes u^{*} T V\right), & \bar{\partial} u=\frac{1}{2}(d u+J \circ d u \circ j) \in \Gamma^{0,1}(u) .
\end{array}
$$

We denote by $D_{V}$ and $D_{g}$ the linearizations of $\bar{\partial}$-operator with respect to the metrics $g_{V}$ and $g$ on $V$, respectively. Since both metrics are Kahler, $D_{V}$ and $D_{g}$ commute with $J$; see $[\mathbf{Z 1}]$.

It should be mentioned that it is not essential for the main gluing construction described in this paper that $(V, J, g)$ is Kahler or even symplectic. If $(V, J, g)$ is not Kahler, we would need to choose an orientation on certain spaces of holomorphic maps and take the induced orientation on the cokernel bundle; see Subsection 3.2. Dropping the Kahler assumption would have almost no effect on the analysis, but would slightly complicate the notation.

\section{Spaces of Bubble Maps}

2.1. Bubble Trees. Let $S$ be either the Riemann sphere $S^{2}$ or a smooth Riemann surface $\Sigma$ of genus at least 2. Allowing the genus-one case would lead to somewhat more complicated notation, but would have no effect on 
the analysis done in Section 3. We put

$$
S^{*}= \begin{cases}S-\{\infty\}, & \text { if } S=S^{2} \\ S, & \text { if } S=\Sigma\end{cases}
$$

Definition 2.1. A bubble tree based on $S$ is a tuple $T=(S, I ; x)$, where

(a) $I$ is a rooted tree and $x: \hat{I} \longrightarrow S \cup S^{2}$ is a map;

(b) $x_{h} \in S^{*}$ if $\iota_{h}=\hat{0}$ and $x_{h} \in S^{2}-\{\infty\}$ otherwise;

(c) if $h_{1} \neq h_{2}$ and $\iota_{h_{1}}=\iota_{h_{2}}, x_{h_{1}} \neq x_{h_{2}}$.

Given a bubble tree $T$ as above, let $\Sigma_{\top}$ be the nodal complex curve

$\Sigma_{\top}=\left((\{\hat{0}\} \times S) \sqcup \bigsqcup_{h \in \hat{I}}\left(\{h\} \times S^{2}\right)\right) / \sim, \quad$ where $\quad(h, \infty) \sim\left(\iota_{h}, x_{h}\right) \forall h \in \hat{I}$.

In other words, the algebraically irreducible components of $\Sigma_{\top}$ are indexed by the set $I$. The point $(h, \infty)$ on the component

$$
\Sigma_{\top, h} \equiv\{h\} \times S^{2}
$$

is attached to the point $\left(\iota_{h}, x_{h}\right)$ on the component $\Sigma_{\top, \iota_{h}}$, where

$$
\Sigma_{\top, \hat{0}}=\{\hat{0}\} \times S .
$$

We will call the component $\Sigma_{\mathrm{T}, \hat{0}}$ corresponding to the root $\hat{0}$ of $I$ the principal component of $\top$ or $\Sigma_{\top}$. For each $i \in \hat{I}, \Sigma_{\top, i}$ will be called the $i$ th bubble component of $\top$ or $\Sigma_{\top}$ or simply a bubble component. Let $\Sigma_{\top, i}^{*}$ and $\Sigma_{\top}^{*}$ denote the open subsets of smooth points of $\Sigma_{\top, i}$ and $\Sigma_{\top}$, respectively, i.e.,

$$
\Sigma_{\top, i}^{*}=\left\{\begin{array}{ll}
\Sigma_{\top, i}-\{(i, \infty)\}-\left\{\left(i, x_{h}\right): \iota_{h}=i\right\}, & \text { if } i \in \hat{I} ; \\
S-\left\{\left(\hat{0}, x_{h}\right): \iota_{h}=\hat{0}\right\}, & \text { if } i=\hat{0} ;
\end{array} \quad \Sigma_{\top}^{*}=\bigcup_{i \in I} \Sigma_{\top}^{*}, i .\right.
$$

The complement of $\Sigma_{\top}^{*}$ in $\Sigma_{\top}$ is the set of the singular points or nodes of $\Sigma_{\top}$.

If $i \in I$ and $h \in \hat{I}$, we put

$$
\top^{(i)}=\left(S, I^{(i)} ;\left.x\right|_{\hat{I}^{(i)}}\right) \quad \text { and } \quad \top^{h}=\left(S, I^{h} ;\left.x\right|_{\hat{I}^{h}}\right) .
$$

These tuples are again bubble trees based on $S$. The complex curve $\Sigma_{\top(i)}$ is obtained from $\Sigma_{\top}$ by dropping all bubble components descendant from the $i$ th bubble component. The curve $\Sigma_{T h}$ is obtained by dropping the $h$ th bubble component along with all bubble components descendant from it.

If $S=S^{2}$ and $h \in \hat{I}$, we denote the inverse exponential map $\phi_{x_{h}}$ defined in (1.6) by $\phi_{\top, h}$. If $z \in \Sigma_{\top, i}$, put

$$
r_{\top, h}(z)= \begin{cases}\left|\phi_{\top, h} z\right|, & \text { if } i=\iota_{h} \text { and } z \neq \infty \\ 100, & \text { otherwise }\end{cases}
$$


If $\delta>0$, let $B_{\top, h}(\delta)=\left\{z \in \Sigma_{\top}: r_{\top, h}(z)<\delta\right\}$. We set

$$
r_{\top}=\min _{h \in \hat{I}}\left(\left|q_{S}^{-1}\left(x_{h}\right)\right|, \min \left\{r_{\top, h}\left(\iota_{l}, x_{l}\right): l \neq h\right\}\right) .
$$

The positive number $r_{\top}$ measures the separation of the nodes of $\Sigma_{\top}$ pairwise and from the point $(\hat{0}, \infty)$ of the principal component $\Sigma_{\top, \hat{0}}$. This point will be a special marked point.

If $S=\Sigma$ and $h \in \hat{I}$ is such that $\iota_{h} \in \hat{I}$, we again let $\phi_{\top, h}$ denote the inverse exponential map $\phi_{x_{h}}$ of (1.6) and define $r_{\top, h}$ and $B_{\top, h}(\delta)$ as above. If $g$ is a Riemannian metric on $\Sigma, \iota_{h}=\hat{0}$, and $z \in \Sigma_{\top, i}$, put

$$
r_{\top, g, h}(z)= \begin{cases}d_{g}\left(x_{h}, z\right), & \text { if } i=\hat{0} \\ 100, & \text { otherwise. }\end{cases}
$$

We denote by $\phi_{\top, g, h}$ the inverse exponential map $\phi_{g, x_{h}}$ of (1.7) and by $B_{\top, g, h}(\delta)$ the ball $B_{g}\left(x_{h}, \delta\right)$. We set

$$
\begin{aligned}
r_{\top} g=\min ( & \min _{\iota_{h}=\hat{0}}\left\{r_{\top, g, h}\left(\iota_{l}, x_{l}\right): l \neq h\right\}, \\
& \left.\min _{\iota_{h} \neq \hat{0}}\left(\left|q_{S}^{-1}\left(x_{h}\right)\right|, \min \left\{r_{\top, h}\left(\iota_{l}, x_{l}\right): l \neq h\right\}\right)\right) .
\end{aligned}
$$

The positive number $r_{\top} g$ measures the separation of the nodes of $\Sigma_{\top}$. We say $g$ is a $\top$-admissible Riemannian metric on $\Sigma$ if there exists $\delta>0$ such that for all $h \in \hat{I}$ with $\iota_{h}=\hat{0}$, the metric $g$ is flat on $B_{\top, g, h}(\delta)$.

2.2. The Basic Gluing Construction. In this subsection, we describe a gluing construction on bubble trees, which is the basis of all the other gluing constructions in this paper. Lemma 2.2 plays a very important role in the next section and in the explicit computations of $[\mathbf{Z 2}]$.

Let $\top=(S, I ; x)$ be a bubble tree. If $h \in \hat{I}$, put

$$
F_{h, \top}^{(0)}=\left\{\begin{array}{ll}
\mathbb{C}, & \text { if } x_{h} \in S^{2} ; \\
T_{x_{h}} \Sigma, & \text { if } x_{h} \in \Sigma,
\end{array} \quad F_{\top}^{(0)}=\bigoplus_{h \in \hat{I}} F_{h, \top}^{(0)} .\right.
$$

If $S=S^{2}$, for any $\delta>0$, put

$$
F_{\top, \delta}^{(0)}=\left\{v=\left(\top, v_{\hat{I}}\right): v_{\hat{I}} \in F_{\top}^{(0)},|v| \equiv \sum_{h \in \hat{I}}\left|v_{h}\right|<\delta\right\} .
$$

Let $\delta_{\top} \in(0,1)$ be such that $8 \delta_{\top}^{\frac{1}{2}}<r_{\top}$. If $S=\Sigma$ and $g$ is an admissible metric on $\Sigma$, put

$$
F_{\top, g, \delta}^{(0)}=\left\{v=\left(\top, v_{\hat{I}}\right): v_{\hat{I}} \in F_{\top}^{(0)},|v|_{g} \equiv \sum_{i_{h}=\hat{0}}\left|v_{h}\right|_{g}+\sum_{i_{h} \neq \hat{0}}\left|v_{h}\right|<\delta\right\},
$$


where $\left|v_{h}\right|_{g}=\left|v_{h}\right|_{g, x_{h}}$. Let $\delta_{\top} g \in(0,1)$ be such that $8\left(\delta_{\top} g\right)^{\frac{1}{2}}<r_{\top} g$ and the metric $g$ is flat on $B_{g}\left(x_{h}, 4\left(\delta_{\top} g\right)^{\frac{1}{2}}\right)$ for all $h \in \hat{I}$ with $\iota_{h}=\hat{0}$.

For each $v \in F_{T_{,} \delta_{\top}}^{(0)}$ if $S=S^{2}$ and $v \in F_{T_{,} \delta_{T} g}^{(0)}$ if $S=\Sigma$, we will construct a bubble tree $T(v)$ and a smooth map

$$
q_{v}: \Sigma_{\top(v)} \longrightarrow \Sigma_{\top} .
$$

The Riemann surface $\Sigma_{\top(v)}$ is obtained from $\Sigma_{\top}$ by replacing the attaching node of the bubble $\Sigma_{\top, h}$ by a thin neck whenever $v_{h} \neq 0$. The map $q_{v}$ simply pinches all these necks. Alternatively, the map $q_{v}$ can be described as a stretching of small neighborhoods of the points $\left(\iota_{h}, x_{h}\right)$ in $\Sigma_{\top_{, \iota_{h}}}$ around the bubbles $\Sigma_{\top, h}$.

First, for every $h \in \hat{I}$ and $v_{h} \in F_{\top, h}^{(0)}$ with

$$
\left|v_{h}\right| \in(0, \delta) \text { if } x_{h} \in S^{2} \quad \text { and } \quad\left|v_{h}\right|_{g} \in(0, \delta) \text { if } x_{h} \in \Sigma,
$$

we define local stretching maps

$$
\begin{array}{ll}
q_{h,\left(x_{h}, v_{h}\right)}: \Sigma_{\mathrm{T}^{\left(\iota_{h}\right)}} \longrightarrow \Sigma_{\mathrm{T}^{(h)}} & \text { if } x_{h} \in S^{2} \quad \text { and } \\
q_{g, h,\left(x_{h}, v_{h}\right)}: \Sigma_{\boldsymbol{\top}^{\left(\iota_{h}\right)}} \longrightarrow \Sigma_{\mathrm{T}^{(h)}} & \text { if } x_{h} \in \Sigma .
\end{array}
$$

These maps will stretch a small neighborhood of the point $\left(\iota_{h}, x_{h}\right)$ in $\Sigma_{\top, \iota_{h}}$ around the bubble $\Sigma_{\top, h}$, which is attached to $\Sigma_{\top\left(\iota_{h}\right)}$ at $\left(\iota_{h}, x_{h}\right)$. If $x_{h} \in S^{2}$, let

$$
\begin{aligned}
& p_{h,\left(x_{h}, v_{h}\right)}^{-}: B_{\top, h}\left(2 \delta_{\top}^{\frac{1}{2}}\right) \longrightarrow \mathbb{C} \cup\{\infty\} \quad \text { and } \\
& p_{h,\left(x_{h}, v_{h}\right)}^{+}: B_{\top, h}\left(2 \delta_{\top}^{\frac{1}{2}}\right) \longrightarrow T_{x_{h}} S^{2}
\end{aligned}
$$

be the maps given by

$$
\begin{aligned}
& p_{h,\left(x_{h}, v_{h}\right)}^{-}(z)=\left(1-\beta_{\left|v_{h}\right|}\left(2\left|\phi_{\top, h} z\right|\right)\right) \overline{\left(\frac{v_{h}}{\phi_{\top, h} z}\right)} \quad \text { and } \\
& p_{h,\left(x_{h}, v_{h}\right)}^{+}(z)=\beta_{\left|v_{h}\right|}\left(\left|\phi_{\top, h} z\right|\right)\left(\phi_{\top, h} z\right) .
\end{aligned}
$$

We note that

$$
\overline{\left(\frac{1}{p_{h,\left(x_{h}, v_{h}\right)}^{-}(z)}\right)}=\frac{\phi_{\top, h} z}{v_{h}} \quad \forall z \in B_{\top, h}\left(\left|v_{h}\right|^{\frac{1}{2}} / 2\right) .
$$

Define $q_{h,\left(x_{h}, v_{h}\right)}: \Sigma_{\top h} \longrightarrow \Sigma_{\top(h)}$ by

$$
q_{h,\left(x_{h}, v_{h}\right)}(z)= \begin{cases}\left(h, q_{S}\left(p_{h,\left(x_{h}, v_{h}\right)}^{-}(z)\right)\right), & \text { if } r_{\top, h}(z) \leq\left|v_{h}\right|^{\frac{1}{2}} \\ \left(\iota_{h}, \phi_{\top, h}^{-1} p_{h,\left(x_{h}, v_{h}\right)}^{+}(z)\right), & \text { if }\left|v_{h}\right|^{\frac{1}{2}} \leq r_{\top, h}(z) \leq 2\left|v_{h}\right|^{\frac{1}{2}} \\ z, & \text { otherwise. }\end{cases}
$$

This map wraps the ball $B_{\top, h}\left(\left|v_{h}\right|^{\frac{1}{2}}\right)$ around the sphere $\Sigma_{\top, h}$. It stretches the ball $B_{\top, h}\left(\left|v_{h}\right|^{\frac{1}{2}} / 2\right)$ by the factor of $1 / v_{h}$, as can be seen from (2.6). The 
map $q_{h,\left(x_{h}, v_{h}\right)}$ is smooth everywhere and is a diffeomorphism, outside of the circle $r_{\mathrm{T}, h}(z)=\left|v_{h}\right|^{\frac{1}{2}}$ in $\Sigma_{\top, \iota_{h}}$.

If $x_{h} \in \Sigma$ and thus $v_{h} \in T_{x_{h}} \Sigma$, similarly to the above, let

$$
\begin{aligned}
& p_{g, h, v_{h}}^{-}: B_{\top, g, h}\left(2 \delta^{\frac{1}{2}}\right) \longrightarrow \mathbb{C} \cup\{\infty\} \quad \text { and } \\
& p_{g, h, v_{h}}^{+}: B_{\top, g, h}\left(2 \delta^{\frac{1}{2}}\right) \longrightarrow T_{x_{h}} \Sigma
\end{aligned}
$$

be the maps given by

$$
\begin{aligned}
& p_{g, h, v_{h}}^{-}(z)=\left(1-\beta_{\left|v_{h}\right|_{g}}\left(2\left|\phi_{\top, g, h} z\right|_{g}\right)\right) \overline{\left(\frac{v_{h}}{\phi_{\top, g, h} z}\right)} \quad \text { and } \\
& p_{g, h, v_{h}}^{+}(z)=\beta_{\left|v_{h}\right|_{g}}\left(\left|\phi_{\top, g, h} z\right|_{g}\right)\left(\phi_{\top, g, h} z\right) .
\end{aligned}
$$

Note that the ratio $v_{h} / \phi_{\top, g, h} z$ is well-defined as an extended complex number, since $T_{x_{h}} \Sigma$ is one-dimensional and $v_{h} \neq 0$. We define

$$
q_{g, h, v_{h}}: \Sigma_{\top h} \longrightarrow \Sigma_{\top(h)}
$$

by

$$
q_{g, h, v_{h}}(z)= \begin{cases}\left(h, q_{S}\left(p_{g, h, v_{h}}^{-}(z)\right)\right), & \text { if } r_{\top, g, h}(z) \leq\left|v_{h}\right|^{\frac{1}{2}} \\ \left.\left(\iota_{h}, \phi_{g, \top, h}^{-1} p_{g, h, v_{h}}^{+}(z)\right)\right), & \text { if }\left|v_{h}\right|^{\frac{1}{2}} \leq r_{\top, g, h}(z) \leq 2\left|v_{h}\right|^{\frac{1}{2}} \\ z, & \text { otherwise. }\end{cases}
$$

Similarly to the case $x_{h} \in S^{2}, q_{g, h, v_{h}}$ is smooth and is a diffeomorphism, except on the circle $r_{\top, g, h}(z)=\left|v_{h}\right|_{g}^{\frac{1}{2}}$ in $\Sigma_{\top, i_{h}}$.

If $S=S^{2}$, for every $h \in I$ and $v \in F_{\top, \delta}^{(0)}$, we now define a bubble tree $\top_{h}(v)$ and a smooth map $q_{v, h}: \Sigma_{\top_{h}(v)} \longrightarrow \Sigma_{\top^{(h)}}$. Choose an ordering of $I$ consistent with its partial ordering. If $h=\hat{0}$, we take

$$
I_{h}(v)=\{\hat{0}\}, \quad \top_{h}(v)=\left(S, I_{h}(v) ;\right), \quad \text { and } \quad q_{v, h}=I d_{S} .
$$

Suppose $h \neq \hat{0}$ and

$$
\top_{h-1}(v)=\left(S, \hat{I}_{h-1}(v) ; x_{h}(v)\right)
$$

with $I_{h-1}(v) \subset I$. If $v_{h}=0$, put

$$
\begin{gathered}
I_{h}(v)=I_{h-1}(v) \cup\{h\}, \\
\left(\iota_{h, l}(v), x_{h, l}(v)\right)= \begin{cases}\left(\iota_{h-1, l}(v), x_{h-1, l}(v)\right), & \text { if } l \in I_{h-1}(v) ; \\
q_{v, \iota_{h}}^{-1}\left(\iota_{h}, x_{h}\right), & \text { otherwise. }\end{cases}
\end{gathered}
$$

Let $\left.q_{v, h}\right|_{\Sigma_{\mathrm{T}_{h-1}(v)}}=q_{v, h-1}$ and $q_{v, h}(h, z)=(h, z)$. If $v_{h} \neq 0$, let

$$
I_{h}(v)=I_{h-1}(v), \quad\left(\iota_{h, l}(v), x_{h, l}(v)\right)=\left(\iota_{h-1, l}(v), x_{h-1, l}(v)\right) .
$$


We take $q_{v, h}=q_{h,\left(x_{h}, v_{h}\right)} \circ q_{v, h-1}$. Inductively this procedure defines a bubble tree $\mathrm{\top}(v)=\top_{h^{*}}(v)$ based on $S$ and a smooth map

$$
q_{v}=q_{v, h^{*}}: \Sigma_{\top(v)} \longrightarrow \Sigma_{\top},
$$

where $h^{*}$ is the largest element of $I$. This map is a diffeomorphism outside of $|I-I(v)|$ disjoint circles. The resulting bubble tree and map are independent of the choice of the extension of the partial ordering. While the domains of the maps $q_{v, h}$ do depend on such a choice, whenever we make use of the maps $q_{v, h}$ below, the result will also be independent of the choice. If $S=\Sigma$, for every $h \in I$ and $v \in F_{\top, g, \delta}^{(0)}$, we define bubble tree $\top_{g, h}(v)$ and maps

$$
q_{g, v, h}: \Sigma_{\top_{g, h}(v)} \longrightarrow \Sigma_{\top(h)}
$$

similarly to the above, but replacing $q_{h,\left(x_{h}, v_{h}\right)}$ by $q_{g, h, v_{h}}$ whenever $\iota_{h}=\hat{0}$. We let $\top_{g}(v)=\top_{g, h^{*}}(v)$ and $q_{g, v}=q_{g, v, h^{*}}$. As before, $q_{g, v}$ is smooth and a diffeomorphism outside of $|I-I(v)|$ disjoint circles.

If $S=S^{2}$ and $v_{h} \neq 0$, put

$$
\begin{aligned}
& A_{v, h}^{+}=q_{v, \iota_{h}}^{-1}\left(\left\{z \in \Sigma_{\top_{, \iota_{h}}}:\left|v_{h}\right|^{\frac{1}{2}} \leq r_{\top, h}(z) \leq 2\left|v_{h}\right|^{\frac{1}{2}}\right\}\right) \\
& A_{v, h}^{-}=q_{v, \iota_{h}}^{-1}\left(\left\{z \in \Sigma_{\top, \iota_{h}}: \frac{1}{2}\left|v_{h}\right|^{\frac{1}{2}} \leq r_{\top, h}(z) \leq\left|v_{h}\right|^{\frac{1}{2}}\right\}\right) .
\end{aligned}
$$

Note that $A_{v, h}^{ \pm} \subset \Sigma_{\top(v), i_{h}^{*}(v)}$, where

$$
i_{h}^{*}(v)=\min \left\{i \in I: i<h \text { and } v_{h^{\prime}} \neq 0 \text { if } i<h^{\prime}<h\right\}=\max \{i \in I(v): i<h\} .
$$

If $S=\Sigma$ and $v_{h} \neq 0$, we similarly define

$$
\begin{aligned}
& A_{g, v, h}^{+}=q_{g, v, \iota_{h}}^{-1}\left(\left\{z \in \Sigma_{\top, \iota_{h}}:\left|v_{h}\right|_{g}^{\frac{1}{2}} \leq r_{\top, g, h}(z) \leq 2\left|v_{h}\right|_{g}^{\frac{1}{2}}\right\}\right) ; \\
& A_{g, v, h}^{-}=q_{g, v, \iota_{h}}^{-1}\left(\left\{z \in \Sigma_{\top, \iota_{h}}: \frac{1}{2}\left|v_{h}\right|_{g}^{\frac{1}{2}} \leq r_{\top, g, h}(z) \leq\left|v_{h}\right|_{g}^{\frac{1}{2}}\right\}\right),
\end{aligned}
$$

where $\left|v_{h}\right|_{g}$ and $r_{\top, g, h}$ denote $\left|v_{h}\right|$ and $r_{\top, h}$ if $\iota_{h} \in \hat{I}$.

Lemma 2.2. If $S=S^{2}$, the map $q_{v}$ is holomorphic outside of the annuli $A_{v, h}^{ \pm}$with $v_{h} \neq 0$. For such $h$ and for all $z \in q_{v, \iota_{h}}\left(A_{v, h}^{-}\right)$,

$$
\begin{gathered}
\left\|d q_{h,\left(x_{h}, v_{h}\right)}\right\|_{C^{0}\left(q_{v, \iota_{h}}\left(A_{v, h}^{ \pm}\right)\right)} \leq C \\
\left.\bar{\partial}\left(q_{v} \circ q_{v, \iota_{h}}^{-1}\right)\right|_{z}=-\left.\left.\left.2\left|v_{h}\right|^{-\frac{1}{2}}\left(\frac{v_{h}}{\phi_{\top, h} z}\right) d q_{S}\right|_{p_{h,\left(x_{h}, v_{h}\right)}} \circ \partial \beta\right|_{2\left|v_{h}\right|^{-\frac{1}{2}} \phi_{\top, h} z} \circ d \phi_{\top, h}\right|_{z},
\end{gathered}
$$

where the norm is computed with respect to the standard metric on $S^{2}$, and $\beta$ is a viewed as a function on $\mathbb{C}$ via the standard norm on $\mathbb{C}$. If $S=\Sigma$, the map $q_{g, v}$ is holomorphic outside of the annuli $A_{g, v, h}^{ \pm}$with $v_{h} \neq 0$. For such $h$ 
and for all $z \in q_{g, v, \iota_{h}}\left(A_{v, h}^{-}\right)$,

$$
\begin{gathered}
\left\|d q_{g, h, v_{h}}\right\|_{C^{0}\left(q_{g, v, \iota_{h}}\left(A_{g, v, h}^{ \pm}\right)\right)} \leq C_{g} \quad \text { if } \quad \iota_{h}=\hat{0} ; \\
\left\|d q_{h,\left(x_{h}, v_{h}\right)}\right\|_{C^{0}\left(q_{g, v, \iota_{h}}\left(A_{g, v, h}^{ \pm}\right)\right)} \leq C \quad \text { if } \quad \iota_{h} \neq \hat{0} ; \\
\left.\bar{\partial}\left(q_{g, v} \circ q_{g, v, \iota_{h}}^{-1}\right)\right|_{z}=-\left.\left.\left.2\left|v_{h}\right|^{-\frac{1}{2}}\left(\frac{v_{h}}{\phi_{\top, h} z}\right) d q_{S}\right|_{p_{\left.h, x_{h}, v_{h}\right)}} \circ \partial \beta\right|_{2\left|v_{h}\right|^{-\frac{1}{2}} \phi_{\top, h} z} \circ d \phi_{\top, h}\right|_{z},
\end{gathered}
$$

where we regard $\beta$ as a function on $T_{x_{h}} \Sigma$ via the metric $g$ and denote $\phi_{g, \top, h}$ by $\phi_{\top, h}$ if $\iota_{h}=\hat{0}$.

Proof. The first statement in each of the two cases is immediate from the construction. The estimates on the differential of $q_{h,\left(x_{h}, v_{h}\right)}$ and $q_{g, h, v_{h}}$ follow from (1.4). Suppose $S=\Sigma, \iota_{h}=\hat{0}, v_{h} \neq 0$, and $z \in A_{g, v, h}^{-}$. Since $q_{g, v}=q_{g, v, \iota_{h}}$ on $A_{g, v, h}^{-}$and $q_{S}$ is anti-holomorphic, from (2.8) and (2.9) we obtain

$$
\begin{aligned}
\left.\bar{\partial} q_{g, v}\right|_{z} & =\left.d q_{S}\right|_{p_{g, h,\left(x_{h}, v_{h}\right)} z} \circ \partial p_{g, h,\left.\left(x_{h}, v_{h}\right)\right|_{z}} \\
& =-\left.\left.\left.2\left|v_{h}\right|_{g}^{-\frac{1}{2}}\left(\frac{v_{h}}{\phi_{\top, g, h} z}\right) d q_{S}\right|_{p_{g, h,\left(x_{h}, v_{h}\right)}} \circ \partial \beta\right|_{2\left|v_{h}\right|_{g}^{-\frac{1}{2}} \phi_{\top, g, h} z} \circ d \phi_{\top, g, h}\right|_{z} .
\end{aligned}
$$

The other cases are proved similarly, since

$$
q_{g, v} \circ q_{g, v, \iota_{h}}^{-1}=q_{h,\left(x_{h}, v_{h}\right)} \quad \text { on } \quad q_{g, v, \iota_{h}}\left(A_{g, v, h}^{-}\right)
$$

and a similar statement holds in the case $S=S^{2}$.

\subsection{Curves with Marked Points.}

\section{Definition 2.3.}

(1) If $M$ is a finite set, a curve with $M$-marked points based on $S$ is a tuple

$$
\mathcal{C}=(S, M, I ; x,(j, y)), \quad \text { where }
$$

(a) $\top_{\mathcal{C}} \equiv(S, I ; x)$ is a bubble tree based on $S$, and $j: M \longrightarrow I$ and $y: M \longrightarrow S \cup S^{2}$ are maps;

(b) $\left(j_{l}, y_{l}\right) \in \Sigma_{\top_{\mathcal{C}}, j_{l}}^{*}$ and $y_{l} \neq \infty$ for all $l \in M$;

(c) for all $l_{1}, l_{2} \in M$ with $l_{1} \neq l_{2}$ and $j_{l_{1}}=j_{l_{2}}, y_{l_{1}} \neq y_{l_{2}}$.

(2) The curve $\mathcal{C}$ is stable if

$$
\left|\left\{h: \iota_{h}=i\right\}\right|+\left|\left\{l: j_{l}=i\right\}\right| \geq 2
$$

for all $i \in \hat{I}$ if $S=\Sigma$ and all $i \in I$ if $S=S^{2}$.

Via the construction in Subsection 2.1, such a tuple $\mathcal{C}$ corresponds to a complex curve $\Sigma_{\mathcal{C}} \equiv \Sigma_{\top_{\mathcal{C}}}$ with marked points $\left\{\left(j_{l}, y_{l}\right)\right\}_{l \in M}$. For each $i \in I$, we denote by $\Sigma_{\mathcal{C}, i}$ and $\Sigma_{\mathcal{C}, i}^{*}$ the surfaces $\Sigma_{T_{\mathcal{C}, i}}$ and $\Sigma_{\top_{\mathcal{C}}, i}^{*}$, respectively. 
With notation as above, for every $h \in \hat{I}$, let $F_{h, \mathcal{C}}^{(0)}$ and $F_{\mathcal{C}}^{(0)}$ denote the spaces $F_{h, T_{\mathcal{C}}}^{(0)}$ and $F_{T_{\mathcal{C}}}^{(0)}$, respectively. If $S=S^{2}$, put

$$
\begin{aligned}
r_{\mathcal{C}}=\min ( & r_{T_{\mathcal{C}}}, \min _{l \in M}\left(\left|q_{S}^{-1} y_{l}\right|,\right. \\
& \left.\left.\min \left\{r_{T_{\mathcal{C}}, h}\left(j_{l}, y_{l}\right): h \in \hat{I}\right\}, \min \left\{\left|\phi_{y_{l}}^{-1} y_{h}\right|: h \neq l, j_{h}=j_{l}\right\}\right)\right) .
\end{aligned}
$$

This positive number measures the minimum pairwise separation between all special points of $\Sigma_{\mathcal{C}}$, including the point $(\hat{0}, \infty)$. Let $\delta_{\mathcal{C}} \in(0,1)$ be such that

$$
16(|I|+|M|) \delta_{\mathcal{C}}^{\frac{1}{2}}<r_{\mathcal{C}} .
$$

If $v=\left(\mathcal{C}, v_{\hat{I}}\right)$ with $v_{\hat{I}} \in F_{\mathcal{C}}^{(0)}$ and $|v|<\delta_{\mathcal{C}}$, we now construct a curve $\mathcal{C}(v)$ with $M$-marked points as follows. Let

$$
\top(v)=(S, I(v) ; x(v)) \quad \text { and } \quad q_{v}: \Sigma_{\top(v)} \longrightarrow \Sigma_{\mathcal{C}}
$$

be the bubble tree and the smooth map defined in Subsection 2.2. Then we take

$$
\mathcal{C}(v)=(S, M, I(v) ; x(v),(j(v), y(v))),
$$

where $\left(j_{l}(v), y_{l}(v)\right) \in \Sigma_{\top}(v), j_{l}(v)$ is defined by

$$
q_{v}\left(j_{l}(v), y_{l}(v)\right)=\left(j_{l}, y_{l}\right) .
$$

Similarly, if $S=\Sigma$ and $g$ is an admissible Riemannian metric on $\Sigma$, put

$$
\begin{array}{r}
r_{\mathcal{C}} g=\min \left(r_{T_{\mathcal{C}}} g, \min _{j_{l}=\hat{0}}\left(\min _{\iota_{h}=\hat{0}}\left\{r_{T_{\mathcal{C}}, g, h}\left(j_{l}, y_{l}\right)\right\}, \min \left\{\left|\phi_{g, y_{l}}^{-1} y_{h}\right|_{g}: h \neq l, j_{h}=\hat{0}\right\}\right),\right. \\
\left.\min _{j_{l} \neq \hat{0}}\left(\left|q_{S}^{-1} y_{l}\right|, \min _{\iota_{h} \neq 0}\left\{r_{T_{\mathcal{C}}, h}\left(j_{l}, y_{l}\right)\right\}, \min \left\{\left|\phi_{y_{l}}^{-1} y_{h}\right|: h \neq l, j_{h}=j_{l}\right\}\right)\right) .
\end{array}
$$

Let $\delta_{\mathcal{C}} g \in(0,1)$ be such that

$$
16(|I|+|M|)\left(\delta_{\mathcal{C}} g\right)^{\frac{1}{2}}<r_{\mathcal{C}} g
$$

and $g$ is flat in $B_{g}\left(x_{h}, 8\left(\delta_{\mathcal{C}} g\right)^{\frac{1}{2}}\right)$ for all $h \in \hat{I}$ with $\iota_{h}=\hat{0}$. If $v \in F_{\mathcal{C}}^{(0)}$ and $|v|_{g}<\delta_{\mathcal{C}} g$, we construct the curve $\mathcal{C}_{g}(v)$ with $M$-marked points in the same way as above, but replacing $q_{v}$ and $\mathrm{\top}(v)$ by $q_{g, v}$ and $\top_{g}(v)$.

Definition 2.4. An isomorphism of curves with $M$-marked points

$$
\mathcal{C}=(S, M, I ; x,(j, y)) \quad \text { and } \quad \mathcal{C}^{\prime}=\left(S, M, I^{\prime} ; x^{\prime},\left(j^{\prime}, y^{\prime}\right)\right)
$$

is a tuple of maps,

$\phi_{0}: I \longrightarrow I^{\prime}, \quad \phi_{1, \hat{0}}: S \longrightarrow S, \quad \phi_{1, h}: S^{2} \longrightarrow S^{2} \quad$ for $h \in I, \quad$ where

(a) $\phi_{0}$ is an isomorphism of the linearly ordered sets $I$ and $I^{\prime}$ and $\phi_{0}\left(j_{l}\right)=j_{l}^{\prime}$ for all $l \in M$;

(b) $\phi_{1, i}$ is a biholomorphic map for all $i \in I$ and $\phi_{1,0}$ is the identity map if $S=\Sigma$; 
(c) $\phi_{1, i}(\infty)=\infty$ for all $i \in I$ if $S=S^{2}$ and for all $i \in \hat{I}$ if $S=\Sigma$;

(d) $\phi_{1, \iota_{h}}\left(x_{h}\right)=x_{\phi_{0}(h)}^{\prime}$ for $h \in \hat{I}$ and $\phi_{1, j_{l}}\left(y_{l}\right)=y_{l}^{\prime}$ for all $l \in M$.

Such a set of maps corresponds to a continuous map

$$
\phi: \Sigma_{\mathcal{C}} \longrightarrow \Sigma_{\mathcal{C}^{\prime}}
$$

that maps the $l$ th marked point $\left(j_{l}, y_{l}\right)$ on $\Sigma_{\mathcal{C}}$ to the $l$ th marked point $\left(j_{l}^{\prime}, y_{l}^{\prime}\right)$ on $\Sigma_{\mathcal{C}^{\prime}}$ and is biholomorphic on each component of $\Sigma_{\mathcal{C}}$. If $S=S^{2}, \phi$ also takes the special marked point $(\hat{0}, \infty)$ on $\Sigma_{\mathcal{C}, \hat{0}}$ to the special marked point $(\hat{0}, \infty)$ on $\Sigma_{\mathcal{C}^{\prime}, \hat{0}}$. Note that if $\mathcal{C}$ is stable, $\mathcal{C}$ has no nontrivial automorphisms.

Let $[\mathcal{C}]$ denote the equivalence class of $\mathcal{C}$ in the set of all curves based on $S$ with marked points. Denote by $\overline{\mathcal{M}}_{S, M}$ the set of all equivalence classes of stable curves based on $S$ with $M$-marked points. If $S=S^{2}, \overline{\mathcal{M}}_{S, M}$ can be identified with the moduli space $\overline{\mathcal{M}}_{0,|M|+1}$ of all stable rational curves with $|M|+1$ marked points, or more canonically with the space $\overline{\mathcal{M}}_{0, M \sqcup\{\hat{0}\}}$ of all stable rational curves with the marked points labeled by the set $M \sqcup\{\hat{0}\}$. If $S=\Sigma$ has genus bigger than two and is generic, $\overline{\mathcal{M}}_{S, M}$ is the closed subset of $\overline{\mathcal{M}}_{g, M}$ consisting of all stable curves of genus $g$ with $M$-marked points that have a fixed complex structure on the principal component. If $S$ has genus two, $\overline{\mathcal{M}}_{S, M}$ is a double cover of the corresponding set for $g=2$, since any smooth genus-two curve has a holomorphic automorphism of order two; see $\left[\mathbf{G H}\right.$, p. 254]. The reason we require that $\phi_{1, \hat{0}}=I d_{\Sigma}$ is that the symplectic invariant of $[\mathbf{R T}]$ disregards the automorphisms of $\Sigma$.

\subsection{Bubble Maps.}

\section{Definition 2.5.}

(1) A $V$-valued bubble map is a tuple $b=(S, M, I ; x,(j, y), u)$, where

(a) $I$ is a linearly ordered set, which is a rooted tree if $S=\Sigma$;

(b) $u: I \longrightarrow C^{\infty}(S ; V) \cup C^{\infty}\left(S^{2} ; V\right)$ is a map;

(c) if $I=\bigsqcup_{k \in K} I_{k}$ is the splitting of $I$ into rooted trees, then $M=\bigsqcup_{k \in K} M_{k}$ for some subsets $M_{k}$ of $M$ such that $\mathcal{C}_{k}=\left(S, M_{k}, I_{k} ;\left.x\right|_{\hat{I}_{k}},\left.(j, y)\right|_{M_{k}}\right)$ is an $M_{k}$-marked curve based on $S$;

(d) $u_{h} \in C^{\infty}(S ; V)$ if $h \in I-\hat{I}, u_{h} \in C^{\infty}\left(S^{2} ; V\right)$ if $h \in \hat{I}$ is a smooth map, and $u_{h}(\infty)=u_{\iota_{h}}\left(x_{h}\right)$ for all $h \in \hat{I}$

(e) for all $i \in \hat{I}$ if $S=\Sigma$ and $i \in I$ if $S=S^{2}$,

$$
\left|\left\{h \in \hat{I}: \iota_{h}=i\right\}\right|+\left|\left\{l \in M: j_{l}=i\right\}\right|<2 \Longrightarrow u_{i *}\left[S^{2}\right] \neq 0 \in H_{2}(V ; \mathbb{Z}) .
$$

(2) The bubble map $b$ is simple if $I$ is a rooted tree; $b$ is $J$-holomorphic if $\bar{\partial}_{J} u_{i}=0$ for all $i \in I$. 
With notation as in Definition 2.5, every bubble map $b$ corresponds to a continuous map

$$
u_{b}: \Sigma_{b} \equiv \bigsqcup_{k \in K} \Sigma_{\mathcal{C}_{k}} \longrightarrow V
$$

which is smooth on the components of $\Sigma_{\mathcal{C}_{k}}$. If $i \in I_{k}$, the restriction of $u_{b}$ to

$$
\Sigma_{b, i} \equiv \Sigma_{\mathcal{C}_{k}, i}
$$

is of course $u_{i}$. If $h \in \hat{I}_{k}$, we put

$$
F_{h, b}^{(0)}=F_{h, \mathcal{C}_{k}}^{(0)} .
$$

Similarly, let

$$
F_{b}^{(0)}=\bigoplus_{k \in K} F_{\mathcal{C}_{k}}^{(0)}, \quad \Sigma_{b}^{*}=\bigcup_{k \in K} \Sigma_{\mathcal{C}_{k}}^{*} \subset \Sigma_{b}, \quad \Sigma_{b, i}^{*}=\Sigma_{\mathcal{C}_{k}, i}^{*} \subset \Sigma_{b, i} .
$$

If $b$ is simple, denote by $\top_{b}$ the bubble tree $\top_{\mathcal{C}_{k}}$ for the unique element $k \in K$.

Definition 2.6. An isomorphism of $V$-valued bubble maps

$$
b=(S, M, I ; x,(j, y), u) \quad \text { and } \quad b^{\prime}=\left(S, M, I^{\prime} ; x^{\prime},\left(j^{\prime}, y^{\prime}\right), u^{\prime}\right)
$$

is a tuple of maps

$\phi_{0}: I \longrightarrow I^{\prime}, \phi_{1, i}: S \longrightarrow S$ for $i \in I-\hat{I}, \phi_{1, i}: S^{2} \longrightarrow S^{2}$ for $i \in \hat{I}, \quad$ where

(a) $\phi_{0}$ is an isomorphism of the linearly ordered sets $I$ and $I^{\prime}$ such that $\phi_{0}\left(j_{l}\right)=j_{l}^{\prime}$ for all $l \in M$;

(b) $\phi_{1, i}$ is a biholomorphic map for all $i \in I$ and is the identity map if $S=\Sigma$ and $i \notin \hat{I}$

(c) $\phi_{1, i}(\infty)=\infty$ for all $i \in I$ if $S=S^{2}$ and for all $i \in \hat{I}$ if $S=\Sigma$;

(d) $\phi_{1, \iota_{h}}\left(x_{h}\right)=x_{\phi_{0}(h)}^{\prime}$ for all $h \in \hat{I}$ and $\phi_{1, j_{l}}\left(y_{l}\right)=y_{l}^{\prime}$ for all $l \in M$;

(e) $u_{\phi_{0}(i)}^{\prime} \circ \phi_{1, i}=u_{i}$ for all $i \in I$.

Such a set of maps corresponds to a continuous map

$$
\phi: \Sigma_{b} \longrightarrow \Sigma_{b^{\prime}}
$$

that maps the marked points of $b$ to the marked points of $b^{\prime}$, intertwines the maps $u_{b}: \Sigma_{b} \longrightarrow V$ and $u_{b^{\prime}}: \Sigma_{b^{\prime}} \longrightarrow V$, and is biholomorphic on each component $\Sigma_{b, i}$ of $\Sigma_{b}$. If $S=S^{2}, \phi$ also takes the special marked point $(\hat{0}, \infty)$ on $\Sigma_{\mathcal{C}, \hat{0}}$ to the special marked point $(\hat{0}, \infty)$ on $\Sigma_{\mathcal{C}^{\prime}, \hat{0}}$.

Let $G_{b}$ denote the group of automorphisms of the bubble map $b$. This group is necessarily finite by the stability condition (e) of Definition 2.5. If 
$\lambda \in H_{2}(V ; \mathbb{Z})$, let

$$
\begin{aligned}
& \bar{C}_{(\lambda ; M)}^{\infty}(S ; V)=\{b=(S, M, I ; x,(j, y), u) \text { is } V \text {-valued bubble map: } \\
& \left.\qquad \sum_{i \in I} u_{i *}\left[\Sigma_{b, i}\right]=\lambda\right\} / \sim ; \\
& C_{(\lambda ; M)}^{\infty}(S ; V)=\left\{b=\left(S,\{\hat{0}\} ;,(\hat{0}, y), u_{\hat{0}}\right) \text { is } V\right. \text {-valued bubble map: } \\
& \left.u_{\hat{0} *}[S]=\lambda\right\} / \sim,
\end{aligned}
$$

where the equivalence relation is given by isomorphisms of $V$-valued bubble maps. If $\mu=\mu_{M}$ is an $M$-tuple of submanifolds of $V$, let

$$
\begin{gathered}
\bar{C}_{(\lambda ; M)}^{\infty}(S ; \mu)=\left\{b=[S, M, I ; x,(j, y), u] \in \bar{C}_{(\lambda ; M)}^{\infty}(S ; V): u_{j_{l}}\left(y_{l}\right) \in \mu_{l} \forall l \in M\right\}, \\
C_{(\lambda ; M)}^{\infty}(S ; \mu)=\left\{b=\left[S, M,\{\hat{0}\} ;,(\hat{0}, y), u_{\hat{0}}\right] \in C_{(\lambda ; M)}^{\infty}(S ; V): u_{\hat{0}}\left(y_{l}\right) \in \mu_{l} \forall l \in M\right\} .
\end{gathered}
$$

A topology on $\bar{C}_{(\lambda ; M)}^{\infty}(S ; V)$ and its subsets $C_{(\lambda ; M)}^{\infty}(S ; V), \bar{C}_{(\lambda ; M)}^{\infty}(S ; \mu)$, and $C_{(\lambda ; M)}^{\infty}(S ; \mu)$ is defined below.

Definition 2.7. Suppose

$$
b^{*}=\left(S, M, I^{*} ; x^{*},\left(j^{*}, y^{*}\right), u^{*}\right) \quad \text { and } \quad b_{k}=\left(S, M, I_{k} ; x_{k},\left(j_{k}, y_{k}\right), u_{k}\right)
$$

be simple bubble maps. If $S=S^{2}$, the sequence $\left\{b_{k}\right\}$ converges to $b^{*}$ if for all $k$ sufficiently large one can choose

(i) $M$-marked curves $\mathcal{C}_{k}=\left(S, M, I^{*} ; x_{k}^{\prime},\left(j^{*}, y^{*}\right)\right)$, and

(ii) elements $\left(v_{k}\right)_{\hat{I}^{*}} \in F_{\mathcal{C}_{k}}^{(0)}$ with $16\left|v_{k}\right|<r_{\mathcal{C}_{k}}^{2}$, such that with $v_{k}=\left(\mathcal{C}_{k},\left(v_{k}\right)_{\hat{I}^{*}}\right)$,

(a) $\lim _{k \longrightarrow \infty} x_{k, h}^{\prime}=x_{h}^{*}$ for all $h \in \hat{I}$, and $\lim _{k \rightarrow \infty}\left|v_{k}\right|=0$;

(b) $\mathcal{C}\left(v_{k}\right)=\left(S, M, I_{k} ; x_{k},\left(j_{k}, y\left(v_{k}\right)\right)\right)$,

$$
\begin{gathered}
\lim _{k \longrightarrow \infty} q_{v_{k}}\left(j_{k, l}, y_{k, l}\right)=\left(j_{l}^{*}, y_{l}^{*}\right) \quad \forall l \in M, \quad \text { and } \\
\lim _{k \longrightarrow \infty} \sup _{z \in \Sigma_{\mathcal{C}\left(v_{k}\right)}} d_{V}\left(u_{b^{*}}\left(q_{v_{k}}(z)\right), u_{b_{k}}(z)\right)=0 .
\end{gathered}
$$

If $S=\Sigma$, convergence is defined in the same way, but $\left|v_{k}\right|$ and $\mathcal{C}\left(v_{k}\right)$ are re-

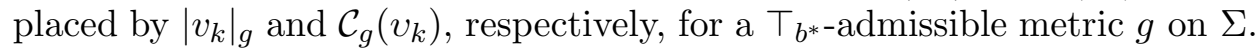

This notion of convergence is independent of the choice of an admissible metric on $\Sigma$. Definition 2.7 induces a topology on the space $\bar{C}_{(\lambda ; M)}^{\infty}(S ; V)$, which will be referred to as the Gromov topology.

Remark. It is often appropriate to strengthen the last condition in (b) above to $L_{1}^{p}$-convergence, for $p>2$, with additional conditions on the behavior near the nodes. However, this is not necessary for the purposes of $[\mathbf{I}]$ and $[\mathbf{Z 2}]$, for example. 
2.5. Strata of Bubble Maps. In this subsection, we introduce the notion of a bubble type. We then define various spaces of holomorphic bubble maps indexed by bubble types and vector bundles over them.

\section{Definition 2.8.}

(1) A bubble type is a tuple $\mathcal{T}=(S, M, I ; j, \underline{\lambda})$ such that

(a) $I$ is a linearly ordered set, and $j: M \longrightarrow I$ and $\underline{\lambda}: I \longrightarrow H_{2}(V ; \mathbb{Z})$ are maps;

(b) for all $i \in \hat{I}$ if $S=\Sigma$ and all $i \in I$ if $S=S^{2}$,

$$
\lambda_{i} \neq 0 \quad \text { if } \quad\left|\left\{h: \iota_{h}=i\right\}\right|+\left|\left\{l: j_{l}=i\right\}\right|<2 ;
$$

(2) Bubble type $\mathcal{T}$ is simple if $I$ is a rooted tree; $\mathcal{T}$ is basic if $\hat{I}=\emptyset$.

(3) Two bubble types $\mathcal{T}=(S, M, I ; j, \underline{\lambda})$ and $\mathcal{T}^{\prime}=\left(S, M, I^{\prime} ; j^{\prime}, \underline{\lambda}^{\prime}\right)$ are equivalent if there exists an isomorphism of linearly ordered sets $\phi_{0}$ : $I \longrightarrow I^{\prime}$ such that $\phi_{0}\left(j_{l}\right)=j_{l}^{\prime}$ for all $l \in M$ and $\lambda_{\phi_{0}(i)}^{\prime}=\lambda_{i}$ for all $i \in I$.

(4) If $\mathcal{T}^{*}=\left(S, M, I^{*} ; j^{*}, \underline{\lambda}^{*}\right)$ and $\mathcal{T}=(S, M, I ; j, \underline{\lambda})$ are two bubble types, $\mathcal{T}^{*}<\mathcal{T}$ if $I \subset I^{*}$,

$$
j_{l}=\max \left\{i \in I: i \leq j_{l}^{*}\right\} \forall l \in M \quad \text { and } \quad \lambda_{i}=\sum_{i=\max \left\{i^{\prime} \in I: i^{\prime} \leq h\right\}} \lambda_{h}^{*} \forall i \in I .
$$

(5) If $\mathcal{T}=(S, M, I ; j, \underline{\lambda})$ is a bubble type, a $\mathcal{T}$-bubble map is a bubble map $b=(S, M, I ; x,(j, y), u)$ such that $u_{i *}\left[\Sigma_{b, i}\right]=\lambda_{i} \in H_{2}(V ; \mathbb{Z})$ for all $i \in I$.

The splitting of $I$ into rooted trees $I_{k}$ induces a splitting of $\mathcal{T}$ into simple bubble types

$$
\mathcal{T}_{k}=\left(S, M_{k}, I_{k} ; j_{k}, \underline{\lambda}_{k}\right),
$$

where $j_{k}$ and $\underline{\lambda}_{k}$ are the restrictions of $j$ and $\underline{\lambda}$ to $M_{k}$ and $I_{k}$, respectively. Similarly, each $\mathcal{T}$-bubble map $b$ corresponds to a $K$-tuple of bubble maps $b_{K}=\left(b_{k}\right)_{k \in K}$, where $b_{k}$ is a $\mathcal{T}_{k}$-bubble map.

We denote the equivalence class of the bubble type $\mathcal{T}$ by $[\mathcal{T}]$ and the group of automorphisms of $\mathcal{T}$ that fix all minimal elements of $I$ by $\mathcal{A}(\mathcal{T})$. This group acts naturally on the set of all $\mathcal{T}$-bubble maps. The partial ordering on the set of bubble types induces a partial ordering on the set of their equivalence classes. If $b$ and $b^{\prime}$ are $\mathcal{T}$ - and $\mathcal{T}^{\prime}$-bubble maps, respectively, such that $[b]=\left[b^{\prime}\right]$, then $[\mathcal{T}]=\left[\mathcal{T}^{\prime}\right]$. Furthermore, if $\left\{b_{k}\right\}$ is a sequence of $\mathcal{T}$-bubble maps, $b^{*}$ is $\mathcal{T}^{*}$-bubble map, and $\left[b_{k}\right]$ converges to $\left[b^{*}\right]$ with respect to the Gromov topology, then $\left[\mathcal{T}^{*}\right] \leq[\mathcal{T}]$.

Let $\mathcal{T}=(S, M, I ; j, \underline{\lambda})$ be a bubble type. We denote by $\langle\mathcal{T}\rangle$ the basic bubble type such that $\langle\mathcal{T}\rangle \geq \mathcal{T}$. It can be described explicitly as follows. Let $I=\bigsqcup_{k \in K} I_{k}$ be the splitting of $I$ into rooted trees and $M=\bigsqcup_{k \in K} M_{k}$ the corresponding splitting of $M$; see Definition 2.5. It can be assumed that 
$K=I-\hat{I}$ and $k$ is the unique minimum element of $I_{k}$. For every $k \in K$ and $l \in M_{k}$, let

$$
\lambda_{k}^{\prime}=\sum_{i \in I_{k}} \lambda_{i}, \quad j_{l}^{\prime}=k .
$$

Then $\langle\mathcal{T}\rangle=\left(S, M, K ; j^{\prime}, \underline{\lambda}^{\prime}\right)$.

Suppose $\mathcal{T}=(S, M, I ; j, \underline{\lambda})$ is a simple bubble type. If $H$ is a subset of $\hat{I}$, we define bubble type $\mathcal{T}(H)=\left(S, M, H \sqcup\{\hat{0}\} ; j^{\prime}, \underline{\lambda}^{\prime}\right)$ by

$$
\begin{gathered}
j_{l}^{\prime}=\max \left\{i \in H \sqcup\{\hat{0}\}: i \leq j_{l}\right\} \quad \text { and } \\
\lambda_{i}^{\prime}=\sum_{i_{H}^{*} \leq h \leq i} \lambda_{h} \text { with } i_{H}^{*}=\max \left\{i^{*} \in H \sqcup \hat{0}: i^{*} \leq i\right\} .
\end{gathered}
$$

Then $\mathcal{T}(H)$ is again a bubble type. The bubble type $\mathcal{T}(H)$ is the bubble type obtained by gluing $\mathcal{T}$-bubble maps with the parameter $v_{\hat{I}}$ such that $v_{h}=0$ if and only if $h \in H$; see the next section.

Given a bubble type $\mathcal{T}=(S, M, I ; j, \underline{\lambda})$, let $d(\mathcal{T}): I \longrightarrow \mathbb{R}$ be given by

$$
d_{i}(\mathcal{T})=\left|\lambda_{i}\right|+\left|\left\{l \in M: j_{l}=i\right\}\right|+\sum_{\iota_{h}=i} d_{h}(\mathcal{T}) \quad \forall i \in I
$$

Since $I$ is a linearly ordered set, the numbers $d_{i}(\mathcal{T})$ are uniquely defined by $(2.12)$. If

$$
b=(S, M, I ; x,(j, y), u)
$$

is a $\mathcal{T}$-bubble map, $b$ is $\mathcal{T}$-balanced if for all $i \in \hat{I}$

$$
\begin{aligned}
& \text { (B1) } \int_{\mathbb{C}}\left|d u_{i} \circ q_{N}\right|^{2} z+\sum_{\iota_{h}=i} d_{h}(\mathcal{T}) x_{h}+\sum_{j_{l}=i} y_{l}=0 ; \\
& \text { (B2) } \int_{\mathbb{C}}\left|d u_{i} \circ q_{N}\right|^{2} \beta(|z|)+\sum_{\iota_{h}=i} d_{h}(\mathcal{T}) \beta\left(\left|x_{h}\right|\right)+\sum_{j_{l}=i} \beta\left(\left|y_{l}\right|\right)=\frac{1}{2} .
\end{aligned}
$$

The integrals above are computed with respect to the metric $g_{V}$ on $V$. Recall that we consider $\mathbb{C}$ to be a subset of $S^{2}$ via the map $q_{N}$. Thus, $x_{h}$ and $y_{l}$ can be viewed as complex numbers, as done above. If $S=S^{2}$ and $b$ is as above, $b$ is completely $\mathcal{T}$-balanced (or $c b$ ) if (B1) and (B2) hold for all $i \in I$.

Denote by $\mathcal{H}_{\mathcal{T}}$ the set of all holomorphic $\mathcal{T}$-bubble maps. Let

$$
P S L_{2}^{(0)}=\left\{g \in P S L_{2}: g(\infty)=\infty\right\}, \quad \mathcal{G}_{\mathcal{T}}=\prod_{h \in \hat{I}} P S L_{2}^{(0)}
$$

The group $\mathcal{G}_{\mathcal{T}}$ acts on $\mathcal{H}_{\mathcal{T}}$ by reparametrizations. In other words, if

$$
b=(S, M, I ; x,(j, y), u) \in \mathcal{H}_{\mathcal{T}} \quad \text { and } \quad g=g_{\hat{I}} \in \mathcal{G}_{\mathcal{T}}
$$


then $g b=(S, M, I ; g x,(j, g y),(g u))$ is defined by

$$
\begin{aligned}
& (g x)_{h}=\left\{\begin{array}{ll}
g_{\iota_{h}} x_{h}, & \text { if } \iota_{h} \in \hat{I} ; \\
x_{h}, & \text { if } \iota_{h} \notin \hat{I} ;
\end{array} \quad(g y)_{l}= \begin{cases}g_{j_{l}} y_{l}, & \text { if } j_{l} \in \hat{I} ; \\
y_{l}, & \text { if } j_{l} \notin \hat{I} ;\end{cases} \right. \\
& (g u)_{i}= \begin{cases}g_{i} \cdot u_{i}, & \text { if } i \in \hat{I} \\
u_{i}, & \text { if } i \notin \hat{I},\end{cases}
\end{aligned}
$$

where for any map $f: S^{2} \longrightarrow V$ and $g \in P S L_{2}$, we define

$$
g \cdot f: S^{2} \longrightarrow V \quad \text { by } \quad\{g \cdot f\}(z)=f\left(g^{-1} z\right) .
$$

Let $\mathcal{M}_{\mathcal{T}}^{(0)} \subset \mathcal{H}_{\mathcal{T}}$ denote the subset of $\mathcal{T}$-balanced holomorphic maps and

$$
G_{\mathcal{T}} \equiv \prod_{h \in \hat{I}} S^{1} \subset \mathcal{G}_{\mathcal{T}}
$$

Since every element of $G_{\mathcal{T}}$ is a map on $I, \mathcal{A}(\mathcal{T})$ acts naturally on $G_{\mathcal{T}}$. The semi-direct product $\mathcal{A}(\mathcal{T}) \ltimes G_{\mathcal{T}}$ acts on $\mathcal{M}_{\mathcal{T}}^{(0)}$ and all the stabilizers are finite. Denote the quotient by $\mathcal{M}_{\mathcal{T}}$, and let

$$
\overline{\mathcal{M}}_{\mathcal{T}}=\bigcup_{\mathcal{T}^{\prime} \leq \mathcal{T}} \mathcal{M}_{\mathcal{T}^{\prime}}
$$

If $\mathcal{A}(\mathcal{T})=\{1\}$, corresponding to the quotient $\mathcal{M}_{\mathcal{T}}=\mathcal{M}_{\mathcal{T}}^{(0)} / G_{\mathcal{T}}$, we obtain $|\hat{I}|$ line (orbi)-bundles

$$
\left\{L_{h} \mathcal{T} \longrightarrow \mathcal{M}_{\mathcal{T}}: h \in \hat{I}\right\}
$$

that carry natural norms:

$$
\left|\left[b, c_{h}\right]\right|=\left|c_{h}\right| \quad \text { if } \quad b \in \mathcal{M}_{\mathcal{T}}^{(0)} \quad \text { and } \quad c_{h} \in \mathbb{C} .
$$

If $\mathcal{A}(\mathcal{T}) \neq\{1\}$, the fiber products and connect sums of the above line bundles taken over each orbit of $\mathcal{A}(\mathcal{T})$ are well-defined. Let $F_{h}^{(0)} \mathcal{T} \longrightarrow \mathcal{M}_{\mathcal{T}}^{(0)}$ be the bundle with the fiber $F_{h, b}^{(0)}$ at $b \in \mathcal{M}_{\mathcal{T}}^{(0)}$, i.e.,

$$
F_{h}^{(0)} \mathcal{T}=\left\{\begin{array}{ll}
\mathcal{M}_{\mathcal{T}}^{(0)} \times \mathbb{C}, & \text { if } x_{h} \in S^{2} ; \\
\pi_{h}^{*} T \Sigma, & \text { if } x_{h} \in \Sigma,
\end{array} \quad \text { where } \pi_{h}(b)=x_{h}\right.
$$

with notation as above. The action of $G_{\mathcal{T}}$ on $\mathcal{M}_{\mathcal{T}}^{(0)}$ lifts to an action on each bundle $F_{h}^{(0)} \mathcal{T}$ by

$$
g \cdot\left(b, v_{h}\right)= \begin{cases}\left(g \cdot b, g_{\iota_{h}} g_{h}^{-1} v_{h}\right), & \text { if } \iota_{h} \in \hat{I} ; \\ \left(g \cdot b, g_{h}^{-1} v_{h}\right), & \text { if } \iota_{h} \notin \hat{I} .\end{cases}
$$

Here and in the rest of the paper, we identify $S^{1}$ with the unit complex numbers in the usual way. Let $F_{h} \mathcal{T}$ be the line orbi-bundle over $\mathcal{M}_{\mathcal{T}}$ given by

$$
F_{h} \mathcal{T}=F_{h}^{(0)} \mathcal{T} / G_{\mathcal{T}}
$$


This bundle has a natural norm unless $\iota_{h}=\hat{0}$ and $S=\Sigma$. In such a case, any metric $g$ on $\Sigma$ induces a norm on $F_{h} \mathcal{T}$. Let

$$
F^{(0)} \mathcal{T}=\bigoplus_{h \in \hat{I}} F_{h}^{(0)} \mathcal{T}, F_{b}^{(0)} \mathcal{T}=\left.F^{(0)} \mathcal{T}\right|_{b} ; F \mathcal{T}=\bigoplus_{h \in \hat{I}} F_{h} \mathcal{T}, F_{[b]}^{(0)} \mathcal{T}=\left.F^{(0)} \mathcal{T}\right|_{[b]} .
$$

Note that if $\mathcal{T}^{*}<\mathcal{T}$, there is a natural splitting

$$
\left(\mathcal{A}\left(\mathcal{T}^{*}\right) \ltimes G_{\mathcal{T}^{*}}\right)=\mathcal{A}(\mathcal{T}) \ltimes\left(G_{\mathcal{T}} \times G\right),
$$

with $G$ determined by $\mathcal{T}$ and $\mathcal{T}^{*}$. Thus, $G_{\mathcal{T}}$ acts on $\mathcal{M}_{\mathcal{T}^{*}}^{(0)}$ and the line bundles $F_{h}^{(0)} \mathcal{T}^{*}$, while $G_{\mathcal{T}^{*}}$ acts on $\mathcal{M}_{\mathcal{T}}^{(0)}$ and $F_{h}^{(0)} \mathcal{T}$.

If $S=S^{2}$, let

$\mathcal{B}_{\mathcal{T}}=\left\{b=(S, M, I ; x,(j, y), u) \in \mathcal{H}_{\mathcal{T}}: b\right.$ is cb; $\left.u_{i_{1}}(\infty)=u_{i_{2}}(\infty) \forall i_{1}, i_{2} \in I-\hat{I}\right\}$.

Denote by $\mathcal{U}_{\mathcal{T}}^{(0)} \subset \mathcal{M}_{\mathcal{T}}$ the quotient $\mathcal{B}_{\mathcal{T}} /\left(\mathcal{A}(\mathcal{T}) \ltimes G_{\mathcal{T}}\right)$. The group

$$
G_{\mathcal{T}}^{*} \equiv \prod_{i \in I-\hat{I}} S^{1}
$$

acts on $\mathcal{U}_{\mathcal{T}}^{(0)}$ and $\mathcal{M}_{\mathcal{T}}$ as follows. If

$$
[b]=\left[\left(S^{2}, M, I ; x,(j, y), u\right)\right] \in \mathcal{M}_{\mathcal{T}} \quad \text { and } \quad g=\left(g_{i}\right)_{i \in I-\hat{I}} \in G_{\mathcal{T}}^{*},
$$

define $g[b]=\left[\left(S^{2}, M, I ; g x,(j, g y), g u\right)\right]$ by

$$
\begin{aligned}
& (g x)_{h}=\left\{\begin{array}{ll}
x_{h}, & \text { if } \iota_{h} \in \hat{I} ; \\
g_{\iota_{h}} x_{h}, & \text { if } \iota_{h} \notin \hat{I} ;
\end{array} \quad(g y)_{l}= \begin{cases}y_{l}, & \text { if } j_{l} \in I ; \\
g_{j_{l}} y_{l}, & \text { if } j_{l} \notin \hat{I} ;\end{cases} \right. \\
& (g u)_{i}= \begin{cases}u_{i}, & \text { if } i \in \hat{I} \\
g_{i} \cdot u_{i}, & \text { if } i \notin \hat{I} .\end{cases}
\end{aligned}
$$

As in the previous paragraph, all stabilizers are finite. Furthermore, this $G_{\mathcal{T}^{*} \text {-action on }} \mathcal{M}_{\mathcal{T}}$ naturally lifts to an action on $\mathcal{M}_{\mathcal{T}}^{(0)}$ and along with the $G_{\mathcal{T}}$-action on $\mathcal{M}_{\mathcal{T}}^{(0)}$ induces an action of $\tilde{G}_{\mathcal{T}} \equiv G_{\mathcal{T}}^{*} \times G_{\mathcal{T}}$ on $\mathcal{M}_{\mathcal{T}}^{(0)}$ as well as on $F_{h}^{(0)} \mathcal{T}$ by

$$
\left(g^{*}, g\right) \cdot\left(b, v_{h}\right)= \begin{cases}\left(\left(g^{*}, g\right) \cdot b, g_{\iota_{h}} g_{h}^{-1} v_{h}\right), & \text { if } \iota_{h} \in \hat{I} \\ \left(\left(g^{*}, g\right) \cdot b, g_{\iota_{h}}^{*} g_{h}^{-1} v_{h}\right), & \text { if } \iota_{h} \notin \hat{I} .\end{cases}
$$

Note that $G_{\mathcal{T}^{\prime}}^{*}=G_{\mathcal{T}}^{*}$ whenever $\mathcal{T}^{\prime} \leq \mathcal{T}$. Let

$$
\mathcal{U}_{\mathcal{T}}=\mathcal{U}_{\mathcal{T}}^{(0)} / G_{\mathcal{T}}^{*}, \quad \overline{\mathcal{U}}_{\mathcal{T}}^{(0)}=\bigcup_{\mathcal{T}^{\prime} \leq \mathcal{T}} \mathcal{U}_{\mathcal{T}^{\prime}}^{(0)}, \quad \overline{\mathcal{U}}_{\mathcal{T}}=\bigcup_{\mathcal{T}^{\prime} \leq \mathcal{T}} \mathcal{U}_{\mathcal{T}^{\prime}}
$$

With respect to the Gromov topology, the space $o v \mathcal{U}_{\mathcal{T}}^{(0)}$ is Hausdorff and compact; see $[\mathbf{R T}]$. Furthermore, $G_{\mathcal{T}}^{*}$ acts continuously on $\mathcal{U}_{\mathcal{T}^{\prime}}^{(0)}$ as can be 
easily seen from Definition 2.7. If follows that $\overline{\mathcal{U}}_{\mathcal{T}}$ is also Hausdorff and compact in the quotient topology. Denote by

$$
\left\{L_{i} \mathcal{T} \longrightarrow \overline{\mathcal{U}}_{\mathcal{T}}: i \in I-\hat{I}\right\}
$$

the line orbi-bundles corresponding to the quotient $\overline{\mathcal{U}}_{\mathcal{T}}=\overline{\mathcal{U}}_{\mathcal{T}}^{(0)} / G_{\mathcal{T}}^{*}$. Let

$$
\begin{gathered}
\mathcal{F}_{h} \mathcal{T}=\left(\left.F_{h}^{(0)} \mathcal{T}\right|_{\mathcal{B}_{\mathcal{T}}}\right) / \tilde{G}_{\mathcal{T}} \longrightarrow \mathcal{U}_{\mathcal{T}}, \quad \mathcal{F}_{h,[b]} \mathcal{T}=\left.\mathcal{F}_{h} \mathcal{T}\right|_{[b]} \\
\mathcal{F} \mathcal{T}=\bigoplus_{h \in \hat{I}} \mathcal{F}_{h} \mathcal{T}, \quad \mathcal{F}_{[b]} \mathcal{T}=\left.\mathcal{F} \mathcal{T}\right|_{[b]} .
\end{gathered}
$$

The line bundles $\mathcal{F}_{h} \mathcal{T}$ have natural norms, defined as in the previous paragraph.

If $\mathcal{T}=(S, M, I ; j, \underline{\lambda})$ is a bubble type and

$$
b=(S, M, I ; x,(j, y), u)
$$

is a $\mathcal{T}$-bubble map, for any $l \in M$, let $\mathrm{ev}_{l}: \mathcal{H}_{\mathcal{T}} \longrightarrow V$ be the map given by

$$
\mathrm{ev}_{l}((S, M, I ; x,(j, y), u))=u_{j_{l}}\left(y_{l}\right) .
$$

This map descends to the quotients defined above and induces continuous maps on the spaces $\overline{\mathcal{M}}_{\mathcal{T}}, \overline{\mathcal{U}}_{\mathcal{T}}^{(0)}$, and $\overline{\mathcal{U}}_{\mathcal{T}}$. If $\mu=\mu_{M}$ is an $M$-tuple of submanifolds in $V$, put

$$
\mathcal{H}_{\mathcal{T}}(\mu)=\left\{b \in \mathcal{H}_{\mathcal{T}}: \operatorname{ev}_{l}(b) \in \mu_{l} \forall l \in M\right\} .
$$

Define spaces $\mathcal{M}_{\mathcal{T}}^{(0)}(\mu), \mathcal{M}_{\mathcal{T}}(\mu), \overline{\mathcal{M}}_{\mathcal{T}}(\mu)$, etc. similarly. If $S=S^{2}$, we define another evaluation map,

$$
\text { ev }: \mathcal{B}_{\mathcal{T}} \longrightarrow V \quad \text { by } \quad \operatorname{ev}\left(\left(S^{2}, M, I ; x,(j, y), u\right)\right)=u_{\hat{0}}(\infty),
$$

where $\hat{0}$ is any minimal element of $I$. This map induces continuous maps on the spaces $\overline{\mathcal{U}}_{\mathcal{T}}^{(0)}$ and $\overline{\mathcal{U}}_{\mathcal{T}}$. If $\mu=\mu_{\tilde{M}}$ is an $\tilde{M}$-tuple of constraints, let

$$
\mathcal{U}_{\mathcal{T}}(\mu)=\left\{b \in \mathcal{U}_{\mathcal{T}}: \operatorname{ev}_{l}(b) \in \mu_{l} \forall l \in \tilde{M} \cap M, \operatorname{ev}(b) \in \mu_{l} \forall l \in \tilde{M}-M\right\}
$$

and define $\mathcal{U}_{\mathcal{T}}^{(0)}(\mu)$, etc. similarly.

\section{The Gluing Construction and the Obstruction Bundle}

3.1. Summary and Notation. We now present a gluing construction on the spaces $\mathcal{M}_{\mathcal{T}}(\mu)$ such that $\mathcal{H}_{\mathcal{T}}$ is a smooth manifold with the tangent bundle isomorphic to the kernel of the linearization of the $\bar{\partial}$-operator, as defined below. The space $\mathcal{H}_{\mathcal{T}}$ is well-known to be smooth if the linearization of the $\bar{\partial}$-operator is surjective; see Chapter 3 in [MS]. However, surjectivity of the linearization is not a necessary condition; see $[\mathbf{Z 2}]$ for examples. In fact, there are two main cases of primary interest to us. The first is when $\mathcal{T}=\left(S^{2}, M, I ; j, \underline{\lambda}\right)$ and the linearization of the $\bar{\partial}$-operator is indeed surjective. In this case, we give an analytic description of a neighborhood of 
$\mathcal{U}_{\mathcal{T}}(\mu)$ in $\overline{\mathcal{U}}_{\langle\mathcal{T}\rangle}(\mu)$ for a generic set of constraints $\mu$. The second case is when $\mathcal{T}=(\Sigma, M, I ; j, \underline{\lambda})$ and the cokernels of the linearization of the $\bar{\partial}$-operator form a vector bundle over $\mathcal{H}_{\mathcal{T}}$, which will be the analogue of Taubes's obstruction bundle of $[\mathbf{T}]$ in the gluing construction below. Using the same analysis as in the first case, we describe any sufficiently nice element of $C_{(\lambda ; M)}^{\infty}(\Sigma ; \mu)$ lying near $\mathcal{M}_{\mathcal{T}}(\mu)$, where $\lambda=\sum \lambda_{i}$. The elements of $\mathcal{M}_{\Sigma, t \nu, \lambda}(\mu)$ lying near $\mathcal{M}_{\mathcal{T}}(\mu)$ will correspond to the zero set of a certain section of the obstruction bundle.

For our gluing construction, we fix a smooth family $\left\{g_{V, b}: b \in \mathcal{M}_{\mathcal{T}}\right\}$ of Kahler metrics on $(V, J)$. We assume that this family is $\left(\mathcal{A}(\mathcal{T}) \ltimes G_{\mathcal{T}}\right)$ invariant if $S=\Sigma$ and $\left(\mathcal{A}(\mathcal{T}) \ltimes \tilde{G}_{\mathcal{T}}\right)$-invariant if $S=S^{2}$. If $b \in \mathcal{M}_{\mathcal{T}}, X, Y \in$ $T_{q} V$, and $u:(D, j) \longrightarrow V$ is a smooth map from a one-dimensional complex manifold, let

$$
\exp _{b, q} X=\exp _{g_{b}, q} X, \quad \nabla^{b}=\nabla^{g_{b}}, \quad \Pi_{b, X} Y=\Pi_{g_{b}, X} Y, \quad D_{b, u}=D_{g_{b}, u} ;
$$

see Subsection 1.3 for more details. If $S=\Sigma$, we also choose a smooth family

$$
\left\{g_{\mathcal{T}, x}: x=(x)_{\left\{h: \iota_{h}=\hat{0}\right\}} ; x_{h} \in \Sigma ; x_{h_{1}} \neq x_{h_{2}} \text { if } h_{1} \neq h_{2}\right\}
$$

of Riemannian metrics on $\Sigma$ such that each metric $g_{\mathcal{T}, x}$ is flat on a neighborhood of $x_{h}$ in $\Sigma$ for all $h \in \hat{I}$ with $\iota_{h}=\hat{0}$. Existence of such a family of metrics is shown in $[\mathbf{F O}]$. If

$$
b=(\Sigma, M, I ; x,(j, y), u) \in \mathcal{H}_{\mathcal{T}},
$$

let $g_{b, \hat{0}}$ denote the metric $g_{\mathcal{T},(x)_{\left\{h: \iota_{h}=\hat{0}\right\}}}$ on $\Sigma$. If $i \in \hat{I}$, we write $g_{b, i}$ for the standard metric on $S^{2}$. Similarly, if $S=S^{2}$, for all $i \in I$, we write $g_{b, i}$ for the standard metric on $S^{2}$.

$$
\begin{aligned}
& \text { If } b=(S, M, I ; x,(j, y), u) \in \mathcal{H}_{\mathcal{T}}, \text { let } \\
& \begin{array}{l}
\Gamma^{\prime}(b)=\bigoplus_{i \in I} \Gamma\left(u_{i}\right) ; \quad \Gamma(b)=\Gamma\left(u_{b}\right)=\left\{\xi_{I} \in \Gamma^{\prime}(b): \xi_{h}(\infty)=\xi_{\iota_{h}}\left(x_{h}\right) \forall h \in \hat{I}\right\} ; \\
\Gamma^{1}(b)=\Gamma^{1}\left(u_{b}\right)=\bigoplus_{i \in I} \Gamma^{1}\left(u_{i}\right) ; \quad \Gamma^{0,1}(b)=\Gamma^{0,1}\left(u_{b}\right)=\bigoplus_{i \in I} \Gamma^{0,1}\left(u_{i}\right) .
\end{array}
\end{aligned}
$$

Define $D_{b}: \Gamma(b) \longrightarrow \Gamma^{0,1}(b)$ by

$$
\left(D_{b} \xi_{I}\right)_{i}=D_{b, u_{i}} \xi_{i} \quad \forall i \in I
$$

We denote the kernel of the operator $D_{b}$ on $\Gamma(b)$ by $\Gamma_{-}(b)$. If $\xi \in \Gamma\left(u_{i}\right)$ or $\xi \in \Gamma^{1}\left(u_{i}\right)$, let $\|\xi\|_{b, C^{k}}$ and $\|\xi\|_{b, 2}$ denote the $C^{k}$ - and $L^{2}$-norms of $\xi$ computed with respect to the metrics $g_{V, b}$ on $V$ and $g_{b, i}$ on $\Sigma_{b, i}$. If $\xi=\xi_{I} \in \Gamma^{\prime}(b)$ or $\xi \in \Gamma^{1}(b)$, put

$$
\|\xi\|_{b, C^{k}}=\sum_{i \in I}\left\|\xi_{i}\right\|_{b, C^{k}},\|\xi\|_{b, 2}=\sum_{i \in I}\left\|\xi_{i}\right\|_{b, 2} .
$$

Let $\pi_{b,-}: \Gamma(b) \longrightarrow \Gamma_{-}(b)$ be the $\left(L^{2}, b\right)$-orthogonal projection map. 
The space $\mathcal{P}_{b} \mathcal{T}$ of perturbations of a bubble map $b$ is the collection of tuples $\sigma=\left(\xi_{\hat{I}} ; w_{\hat{I} \sqcup M}\right)$, where

$$
\xi_{i} \in \Gamma\left(u_{i}\right) \forall i \in I, \quad w_{h} \in F_{h, b}^{(0)} \forall h \in \hat{I}, \quad w_{l} \in \begin{cases}\mathbb{C}, & \text { if } l \in M \& \Sigma_{b, j_{l}}=S^{2} \\ T_{y_{l}} \Sigma, & \text { if } l \in M \& \Sigma_{b, j_{l}}=\Sigma .\end{cases}
$$

If $\sigma$ is sufficiently small, we define $\exp _{b} \sigma=\left(S, M, I ; x(\sigma),(j, y(\sigma)), u_{\sigma}\right)$ by

$$
\begin{gathered}
x_{h}(\sigma)= \begin{cases}x_{h}+w_{h}, & \text { if } \Sigma_{b, i_{h}}=S^{2} ; \\
\exp _{g_{b, 0}, x_{h}} w_{h}, & \text { if } \Sigma_{b, i_{h}}=\Sigma ;\end{cases} \\
y_{l}(\sigma)=\left\{\begin{array}{ll}
y_{l}+w_{l}, & \text { if } \Sigma_{b, j_{l}}=S^{2} ; \\
\exp _{g_{b, 0}, y_{l}} w_{l}, & \text { if } \Sigma_{b, j_{l}}=\Sigma ;
\end{array} \text { and } u_{\sigma, i}=\exp _{b, u_{i}} \xi_{i} .\right.
\end{gathered}
$$

If $z \in \Sigma$, let $|v|_{b}=|v|_{g_{b, 0}, x}$. For consistency, if $v \in \mathbb{C}$, let $|v|_{b}=|v|$. Along with the $\left(L^{2}, b\right)$-norm on the vector fields defined above, we obtain an innerproduct on the space of tuples $\sigma$ as above.

In order to get a good description of the spaces $\mathcal{M}_{\mathcal{T}}^{(0)}$ as submanifolds of $\mathcal{H}_{\mathcal{T}}$, we describe an action of an open subset of 0 in $(\mathbb{C} \oplus \mathbb{R} \oplus \mathbb{R})^{\hat{I}}$ on bubble maps and distinguished elements $\sigma_{(b, i)}^{(k)} \in \mathcal{P}_{b} \mathcal{T}$ that correspond to this action. If

$$
(c, r, \theta)=(c, r, \theta)_{\hat{I}} \in(\mathbb{C} \times \mathbb{R} \times \mathbb{R})^{\hat{I}}
$$

and $b$ is a bubble map as above, we define

$$
(c, r, \theta) \cdot b=(S, M, I ;(c, r, \theta) x,(j,(c, r, \theta) y),(c, r, \theta) u)
$$

by setting

$$
\begin{gathered}
((c, r, \theta) x)_{h}=e^{i \theta_{\iota_{h}}}\left(1+r_{\iota_{h}}\right)\left(x_{h}+c_{\iota_{h}}\right), \quad((c, r, \theta) y)_{l}=e^{i \theta_{j_{l}}\left(1+r_{j_{l}}\right)\left(y_{l}+c_{j_{l}}\right),} \\
((c, r, \theta) u)_{i}\left(q_{N}(z)\right)=u_{i}\left(q_{N}\left(\left(1+r_{i}\right)^{-1} e^{-i \theta_{i}} z-c_{i}\right)\right) .
\end{gathered}
$$

If $(c, r, \theta)$ is sufficiently small, $(c, r, \theta) \cdot b$ is again a bubble map, i.e., the maps into $V$ still agree at the nodes, and the nodes and the marked points are still all distinct. In fact, the values of the maps at the nodes or the marked points do not change, i.e.,

$$
\begin{gathered}
((c, r, \theta) u)_{\iota_{h}}\left(((c, r, \theta) x)_{h}\right)=u_{\iota_{h}}\left(x_{h}\right), \quad((c, r, \theta) u)_{h}(\infty)=u_{h}(\infty), \\
\quad \text { and } \quad((c, r, \theta) u)_{j_{l}}\left(((c, r, \theta) y)_{l}\right)=u_{j_{l}}\left(y_{l}\right) .
\end{gathered}
$$

Furthermore, if $b \in \mathcal{H}_{\mathcal{T}},(c, r, \theta) \cdot b \in \mathcal{H}_{\mathcal{T}}$. If $b$ is of type $\mathcal{T}$, the above describes the action of a neighborhood of the identity in $\mathcal{G}_{\mathcal{T}}$ on the space of stable maps of type $\mathcal{T}$. The action by $\mathbb{C}$ corresponds to the translations of $\mathbb{C}$, by the first $\mathbb{R}$-component to dilations about the origin, and by the last $\mathbb{R}$ component to rotations about the origin. If $S=S^{2}$ and $(c, r, \theta) \in(\mathbb{C} \times \mathbb{R} \times \mathbb{R})^{I}$ is sufficiently small, we define $(c, r, \theta) \cdot b$ similarly. 
If $u \in C^{\infty}\left(S^{2} ; V\right)$, define $\xi_{u}^{(1)}, \ldots, \xi_{u}^{(4)} \in \Gamma(u)$ by:

$$
\begin{gathered}
\xi_{u}^{(1)}\left(q_{N}(z)\right)=-\left.d\left(u \circ q_{N}\right)\right|_{z} \frac{\partial}{\partial s}, \quad \xi_{u}^{(2)}\left(q_{N}(z)\right)=-\left.d\left(u \circ q_{N}\right)\right|_{z} \frac{\partial}{\partial t} \\
\xi_{u}^{(3)}\left(q_{N}(z)\right)=-\left.d\left(u \circ q_{N}\right)\right|_{z}\left(s \frac{\partial}{\partial s}+t \frac{\partial}{\partial t}\right)=-\left.r d\left(u \circ q_{N}\right)\right|_{z} \frac{\partial}{\partial r}, \\
\xi_{u}^{(4)}\left(q_{N}(z)\right)=\left.d\left(u \circ q_{N}\right)\right|_{z}\left(t \frac{\partial}{\partial s}-s \frac{\partial}{\partial t}\right)=-\left.d\left(u \circ q_{N}\right)\right|_{z} \frac{\partial}{\partial \theta} .
\end{gathered}
$$

where we write $z=s+i t \in \mathbb{C}$ and $r=\sqrt{s^{2}+t^{2}}$. These vector fields extend smoothly by zero over the south pole. For any $x \in S^{2}-\{\infty\}$, let $w_{x}^{(1)}, \ldots, w_{x}^{(4)} \in \mathbb{C}$ be given by

$$
w_{x}^{(1)}=1, \quad w_{x}^{(2)}=i, \quad w_{x}^{(3)}=x, \quad w_{x}^{(4)}=i x .
$$

If $b$ is a bubble map as above, $k=1, \ldots, 4, i^{*} \in \hat{I}$ if $S=\Sigma$ and $i^{*} \in I$ if $S=S^{2}$, let

be given by

$$
\sigma_{\left(b, i^{*}\right)}^{(k)}=\left(\left(\xi_{\left(b, i^{*}\right)}^{(k)}\right)_{I},\left(w_{\left(b, i^{*}\right)}^{(k)}\right)_{\hat{I} \sqcup M}\right)
$$

$$
\begin{gathered}
\xi_{\left(b, i^{*}\right), i}^{(k)}=\left\{\begin{array}{ll}
\xi_{u_{i}}^{(k)}, & \text { if } i=i^{*} ; \\
0, & \text { if } i \neq i^{*} ;
\end{array} \quad w_{\left(b, i^{*}\right), h}^{(k)}= \begin{cases}w_{x_{h}}^{(k)}, & \iota_{h}=i^{*} ; \\
0, & \iota_{h} \neq i^{*} ;\end{cases} \right. \\
w_{\left(b, i^{*}\right), l}^{(k)}= \begin{cases}w_{y_{l}}^{(k)}, & j_{l}=i^{*} ; \\
0, & j_{l} \neq i^{*} .\end{cases}
\end{gathered}
$$

The tuples $\sigma_{\left(b, i^{*}\right)}^{(k)}$ correspond to the infinitesimal action of $\mathcal{G}_{\mathcal{T}}$ on the space of stable maps of type $\mathcal{T}$.

Finally, if $X$ is any space, $F \longrightarrow X$ a normed vector bundle, and $\delta: X \longrightarrow \mathbb{R}$ is any function, let

$$
F_{\delta}=\left\{(b, v) \in F:|v|_{b}<\delta(b)\right\} .
$$

Similarly, if $\Omega$ is a subset of $F$, let $\Omega_{\delta}=F_{\delta} \cap \Omega$. If $v=(b, v) \in F$, denote by $b_{v}$ the image of $v$ under the bundle projection map, i.e., $b$ in this case.

3.2. The Basic Setup. In this subsection, we describe our assumptions on the smooth structure of $\mathcal{H}_{\mathcal{T}}$ and state some of their implications.

Definition 3.1. Bubble type $\mathcal{T}=\left(S^{2}, M, I ; j, \underline{\lambda}\right)$ is $(V, J)$-regular if for all

$$
b=(S, M, I ; x,(j, y), u) \in \mathcal{H}_{\mathcal{T}},
$$

(a) $D_{b, u_{i}}: \Gamma\left(u_{i}\right) \longrightarrow \Gamma^{0,1}\left(u_{i}\right)$ is onto for all $i \in I$;

(b) $\operatorname{ker} D_{b, u_{i}} \longrightarrow T_{u_{i}(\infty)} V, \xi \longrightarrow \xi(\infty)$, is onto for all $i \in I$.

Definition 3.2. Simple bubble type $\mathcal{T}=(S, M, I ; j, \underline{\lambda})$ is $(V, J)$-semiregular if 
(a) the space $\mathcal{H}_{\left(S, \emptyset,\{\hat{0}\} ;, \lambda_{\hat{0}}\right)}$ is a complex manifold, and there exist

$$
\delta, C \in C^{\infty}\left(\mathcal{H}_{\left(S, \emptyset,\{\hat{0}\} ;, \lambda_{\hat{0}}\right)} ; \mathbb{R}^{+}\right)
$$

and for each element $b=\left(S, \emptyset,\{\hat{0}\} ;, u_{\hat{0}}\right)$ of $\mathcal{H}_{\left(S, \emptyset,\{\hat{0}\} ;, \lambda_{\hat{0}}\right)}$ a map

$$
h_{\mathcal{T}, \hat{0} ; b}:\left\{\xi \in \operatorname{ker} D_{g_{V}, b}:\|\xi\|_{g_{V}, C^{0}}<\delta(b)\right\} \longrightarrow \Gamma\left(u_{\hat{0}}\right)
$$

such that

$$
\begin{gathered}
\left\|h_{\mathcal{T}, \hat{0} ; b}(\xi)\right\|_{g_{V}, b} \leq C(b)\|\xi\|_{g_{V}, C^{0}}^{2} \quad \text { and } \\
\left\|h_{\mathcal{T}, \hat{0} ; b}(\xi)-h_{\mathcal{T}, \hat{0} ; b}\left(\xi^{\prime}\right)\right\|_{g_{V}, C^{0}} \leq C(b)\left\|\xi-\xi^{\prime}\right\|_{g_{V}, C^{0}},
\end{gathered}
$$

for all $\xi, \xi^{\prime} \in \operatorname{ker} D_{g_{V}, b}$ with $\|\xi\|_{g_{V}, C^{0}},\left\|\xi^{\prime}\right\|_{g_{V}, C^{0}}<\delta(b)$ and the map

$$
\begin{gathered}
H_{\mathcal{T}, \hat{0} ; b}:\left\{\xi \in \operatorname{ker} D_{g_{V}, b}:\|\xi\|_{g_{V}, C^{0}}<\delta(b)\right\} \longrightarrow \mathcal{H}_{\left(S, \emptyset,\{\hat{0}\} ;, \lambda_{\hat{0}}\right)}, \\
\xi \longrightarrow \exp _{g_{V}, u_{\hat{0}}}\left(\xi+h_{\mathcal{T}, \hat{0} ; b}(\xi)\right)
\end{gathered}
$$

is an orientation-preserving diffeomorphism onto an open neighborhood of $b$ in $\mathcal{H}_{\left(S, \emptyset,\{\hat{0}\} ;, \lambda_{\hat{0}}\right)}$. Furthermore, the family of maps $\left\{H_{\mathcal{T}, \hat{0} ; b}\right.$ : $\left.b \in \mathcal{H}_{\left(S, \emptyset,\{\hat{0}\} ;, \lambda_{\hat{0}}\right)}\right\}$ is smooth.

(b) For all $b=(S, M, I ; x,(j, y), u) \in \mathcal{H}_{\mathcal{T}}$

(b-i) $D_{b, u_{h}}: \Gamma\left(u_{h}\right) \longrightarrow \Gamma^{0,1}\left(u_{h}\right)$ is onto for all $h \in \hat{I}$;

(b-ii) $\operatorname{ker} D_{b, u_{h}} \longrightarrow T_{u_{h}(\infty)} V, \xi \longrightarrow \xi(\infty)$, is onto for all $h \in \hat{I}$.

\section{Remarks.}

(1) All conditions in both definitions above are independent of the choice of metric on $V$.

(2) Condition (a) of Definition 3.2 says that $\mathcal{H}_{\left(S, \emptyset,\{\hat{0}\} ;, \lambda_{\hat{0}}\right)}$ is a smooth manifold modeled on $\operatorname{ker} D_{b}$ for $b \in \mathcal{H}_{\left(S, \emptyset,\{\hat{0}\} ;, \lambda_{\hat{0}}\right)}$, as would be the case if

$$
D_{b}: \Gamma\left(u_{b}\right) \longrightarrow \Gamma^{0,1}\left(u_{b}\right)
$$

were surjective.

(3) The conditions of Definitions 3.1 and 3.2 insure that $\mathcal{H}_{\mathcal{T}}$ is a smooth manifold; see Proposition 3.3 below. However, (b) of Definition 3.1 and (b-ii) of Definition 3.2 are somewhat stronger than necessary to show that $\mathcal{H}_{\mathcal{T}}$ is smooth. They allow us to obtain the second part of (1) in Proposition 3.3, which is used in the proof of surjectivity of the gluing map; see Subsection 4.3. These two conditions hold for all complex homogeneous manifolds; see Section 10 in $[\mathbf{R T}]$.

Note that if $\mathcal{T}$ is semiregular, the homotopy invariance of the index implies that the vector spaces

$$
\Gamma_{-}^{0,1}(b) \equiv \operatorname{coker} D_{b} \approx \operatorname{ker} D_{b}^{*} \subset \Gamma^{0,1}(b), \quad b \in \mathcal{H}_{\mathcal{T}},
$$


form a vector bundle over $\mathcal{H}_{\mathcal{T}}$. Here $D_{b}^{*}$ denotes the formal adjoint of $D_{b}$ with respect to a metric $g$ on $S$; it is a $J$-linear operator. The space ker $D_{b}^{*}$ is independent of a conformal choice of the metric $g$. The bundle $\Gamma_{-}^{0,1} \longrightarrow \mathcal{H}_{\mathcal{T}}$ will be called the $\mathcal{T}$-cokernel bundle. It is $\left(\mathcal{A}(\mathcal{T}) \ltimes G_{\mathcal{T}}\right)$-equivariant, and thus descends to a bundle $\Gamma_{-}^{0,1} \longrightarrow \mathcal{M}_{\mathcal{T}}$, which will be the analogue of Taubes's obstruction in our gluing setting.

Let $\mathcal{T}=(S, M, I ; j, \underline{\lambda})$ be a bubble type. If $b=(S, M, I ; x,(j, y), u) \in$ $\mathcal{H}_{\mathcal{T}}$, put

$$
\begin{aligned}
& \mathcal{K}_{b} \mathcal{T}=\left\{\sigma=\left(\xi, w_{\hat{I} \sqcup M}\right) \in \mathcal{P}_{b} \mathcal{T}:\right. \xi_{i} \in \operatorname{ker}\left(D_{b, u_{i}}\right) \forall i \in I ; \\
&\left\langle\sigma, \sigma_{(b, h)}^{(k)}\right\rangle=0 \forall h \in \hat{I}, k \in[4] ; \\
&\left.\xi_{h}(\infty)=\xi_{\iota_{h}}\left(x_{h}\right)+\left.d u_{\iota_{h}}\right|_{x_{h}} w_{h} \forall h \in \hat{I}\right\} .
\end{aligned}
$$

If $\sigma=\left(\xi, w_{\hat{I} \sqcup M}\right) \in \mathcal{K}_{b} \mathcal{T}$, let

$$
\|\sigma\|_{b, C^{k}}=\|\xi\|_{b, C^{k}}+\sum_{h \in \hat{I}}\left|w_{h}\right|_{b}+\sum_{l \in M}\left|w_{l}\right|_{b}
$$

We take the default norm on $\mathcal{K}_{b} \mathcal{T}$ to be given by $\|\cdot\|_{b, C^{0}}$. If $b$ is as above,

$$
b^{\prime}=\left(S, M, I ; x^{\prime},\left(j, y^{\prime}\right), u\right),
$$

and $\delta>0$, we say $d\left(b, b^{\prime}\right)<\delta$ if there exists $\sigma \in \mathcal{P}_{b} \mathcal{T}$ such that $\exp _{b} \sigma=b^{\prime}$ and $\|\sigma\|_{b, C^{0}} \leq \delta$.

\section{Proposition 3.3.}

(1) If $\mathcal{T}=(S, M, I ; j, \underline{\lambda})$ is a regular or semiregular bubble type, $\mathcal{H}_{\mathcal{T}}$ is a complex manifold and there exist $\epsilon_{\mathcal{T}}, C_{\mathcal{T}} \in C^{\infty}\left(\mathcal{M}_{\mathcal{T}}^{(0)} ; \mathbb{R}^{+}\right)$with the following property. If $b^{*} \in \mathcal{H}_{\mathcal{T}}$ and

$$
b=(S, M, I ; x,(j, y), u) \quad \text { is s.t. } \quad d\left(b^{*}, b\right)<\epsilon_{\mathcal{T}}\left(b^{*}\right) \text { and } \bar{\partial} u_{i}=0 \forall i \in I,
$$

there exist $\xi_{i} \in \Gamma\left(u_{i}\right)$ for $i \in \hat{I}$ such that

$$
\begin{gathered}
\left\|\xi_{i}\right\|_{g_{V}, C^{0}} \leq C_{\mathcal{T}}\left(b^{*}\right) \sum_{h \in \hat{I}} d_{V}\left(u_{\iota_{h}}\left(x_{h}\right), u_{h}(\infty)\right) \quad \text { and } \\
b^{\prime}=\left(S, M, I ; x,(j, y), u^{\prime}\right) \in \mathcal{H}_{\mathcal{T}}
\end{gathered}
$$

where $u_{\hat{0}}^{\prime}=u_{\hat{0}}$ and $u_{i}^{\prime}=\exp _{g_{V}, u_{i}} \xi_{i}$ if $i \in \hat{I}$.

(2) The space $\mathcal{M}_{\mathcal{T}}^{(0)}$ is a smooth oriented manifold on which the group $G_{\mathcal{T}}$ acts smoothly. The maps

$$
\begin{array}{ll}
\mathrm{ev}: \mathcal{M}_{\mathcal{T}}^{(0)} \longrightarrow V, & \operatorname{ev}(S, M, I ; x,(j, y), u)=u_{\hat{0}}(\infty), \\
\mathrm{ev}_{l}: \mathcal{M}_{\mathcal{T}}^{(0)} \longrightarrow V, & \operatorname{ev}_{l}(S, M, I ; x,(j, y), u)=u_{j_{l}}\left(y_{l}\right), \\
\left.d u_{i}\right|_{z}: \mathcal{M}_{\mathcal{T}}^{(0)} \longrightarrow T^{*} \Sigma_{\mathcal{T}, i} \otimes u_{i}^{*} T V, & \left.d u_{i}\right|_{z}(S, M, I ; x,(j, y), u)=\left.d u_{i}\right|_{z},
\end{array}
$$


are smooth. In particular, $u_{i} \longrightarrow\left\|d u_{i}\right\|_{b, C^{0}}$ defines a continuous function on $\mathcal{M}_{\mathcal{T}}^{(0)}$.

(3) There exist $\delta_{\mathcal{T}}, C_{\mathcal{T}} \in C^{\infty}\left(\mathcal{M}_{\mathcal{T}}^{(0)} ; \mathbb{R}^{+}\right)$and smooth maps

$$
h_{\mathcal{T}, b}=h_{\mathcal{T}, b}^{(1)} \oplus h_{\mathcal{T}, b}^{(2)}: \mathcal{K}_{b} \mathcal{T}_{\delta_{\mathcal{T}}(b)} \longrightarrow \Gamma^{\prime}(b) \oplus(\mathbb{C} \oplus \mathbb{R})^{\hat{I}}
$$

such that $\left\|h_{\mathcal{T}, b}(\sigma)\right\|_{b, C^{0}} \leq C_{\mathcal{T}}(b)\|\sigma\|_{b, C^{0}}^{2}$,

$\left\|h_{\mathcal{T}, b}(\sigma)-h_{\mathcal{T}, b}\left(\sigma^{\prime}\right)\right\|_{b, C^{0}} \leq C_{\mathcal{T}}(b)\left(\|\sigma\|_{b, C^{0}}+\left\|\sigma^{\prime}\right\|_{b, C^{0}}\right)\left\|\sigma-\sigma^{\prime}\right\|_{b, C^{0}}$,

and each map

$$
\begin{gathered}
H_{\mathcal{T}, b}^{(0)}:\left\{(\sigma, \theta) \in \mathcal{K}_{b} \mathcal{T}_{\delta_{\mathcal{T}}(b)} \times \mathbb{R}^{\hat{I}}:|\theta|<\pi\right\} \longrightarrow \mathcal{M}_{\mathcal{T}}^{(0)}, \\
H_{\mathcal{T}, b}^{(0)}(b, \sigma, \theta)=\left(h_{\mathcal{T}, b}^{(2)}(\sigma), \theta\right) \cdot \exp _{b}\left(\sigma+h_{\mathcal{T}, b}^{(1)}(\sigma)\right),
\end{gathered}
$$

is orientation-preserving diffeomorphism onto an open neighborhood of $b$ in $\mathcal{M}_{\mathcal{T}}^{(0)}$.

Proof. (1) Let

$$
\mathcal{T}_{i}=\left(\Sigma_{\mathcal{T}, i},\left\{l: j_{l}=i\right\}+\left\{h: \iota_{h}=i\right\},\{\hat{0}\} ; \hat{0}, \lambda_{i}\right) .
$$

By (a) of Definition 3.1, and (a) and (b-i) of Definition 3.2, $\mathcal{H}_{\mathcal{T}_{i}}$ is a complex manifold for all $i \in I$. Let

$$
\triangle_{V}^{\hat{I}}=\left\{(q, q)_{\hat{I}} \in \prod_{\hat{I}}(V \times V): q_{h} \in V\right\} .
$$

The submanifold $\triangle_{V}^{\hat{I}}$ is the $\hat{I}$-product of the diagonal in $V \times V$. Since $V$ is oriented, so is the normal bundle of $\triangle_{V}^{\hat{I}}$. Claim (1) of the proposition follows by applying the Implicit Function Theorem, (b) of Definition 3.1 and (b-ii) of Definition 3.2 to the smooth map

$$
\begin{gathered}
\operatorname{ev}_{\hat{I}}: \prod_{i \in I} \mathcal{H}_{\mathcal{T}_{i}} \longrightarrow \prod_{\hat{I}}(V \times V), \\
\operatorname{ev}_{h}((S, M, I ; x,(j, y), u))=\left(u_{h}(\infty), u_{\iota_{h}}\left(x_{h}\right)\right) .
\end{gathered}
$$

Note that $\mathcal{H}_{\mathcal{T}}=\mathrm{ev}_{\hat{I}}^{-1}\left(\triangle_{V}^{\hat{I}}\right)$.

(2) For any $u \in C^{\infty}\left(S^{2} ; V\right)$, define $\tilde{\Psi} u \in \mathbb{C}, \Psi^{(3)} u \in \mathbb{R}$, and $\Psi u \in \mathbb{C} \times \mathbb{R}$ by

$$
\Psi u=\left(\tilde{\Psi} u, \Psi^{(3)} u\right)=\left(\int_{\mathbb{C}}\left|d u \circ q_{N}\right|^{2} z, \int_{\mathbb{C}}\left|d u \circ q_{N}\right|^{2} \beta(|z|)-\frac{1}{2}\right),
$$


where the integrals are computed using the metric $g_{V}$. For $i^{*} \in \hat{I}$ if $S=\Sigma$ and $i^{*} \in I$ if $S=S^{2}$, we define maps

$$
\begin{gathered}
\Psi_{\mathcal{T}, i^{*}}: \prod_{i \in I} \mathcal{H}_{\mathcal{T}_{i}} \longrightarrow \mathbb{C} \times \mathbb{R} \quad \text { by } \\
\Psi_{\mathcal{T}, i^{*}}(S, M, I ; x,(j, y), u)=\left(\tilde{\Psi} u_{i^{*}}+\sum_{\iota_{h}=i^{*}} d_{h}(\mathcal{T}) x_{h}+\sum_{j_{l}=i^{*}} y_{l},\right. \\
\left.\Psi^{(3)} u_{i^{*}}+\sum_{\iota_{h}=i^{*}} d_{h}(\mathcal{T}) \beta\left(\left|x_{h}\right|\right)+\sum_{j_{l}=i^{*}} \beta\left(\left|y_{l}\right|\right)\right) .
\end{gathered}
$$

These maps $\Psi_{\mathcal{T}_{, i *}}$ are smooth, since the smooth structure on all $\mathcal{H}_{\mathcal{T}_{i}}$ is described similarly to (a) of Definition 3.2. Furthermore, if $b \in \mathcal{M}_{\mathcal{T}}^{(0)}, i^{*} \in \hat{I}$, and $k^{*}=1,2,3$, since $\Psi_{\mathcal{T}, i}(b)=0$ for all $i$ and $\beta^{\prime}$ does not change sign, by Lemma 3.4,

$$
\left.d \Psi_{\mathcal{T}, i^{*}}^{\left(k^{*}\right)}\right|_{b} \sigma_{(b, i)}^{(k)} \begin{cases}=0, & \text { if } i \neq i^{*} \\ \neq 0, & \text { if } i=i^{*}, k=k^{*} \\ =0, & \text { if } k \neq k^{*} \neq 3\end{cases}
$$

where $k=1,2,3$. By (b) of Definition 3.1 and (b-ii) of Definition 3.2, it follows that the map

$$
\prod_{i \in I} \mathcal{H}_{\mathcal{T}_{i}} \longrightarrow(\mathbb{C} \times \mathbb{R})^{\hat{I}} \times \prod_{\hat{I}}(V \times V), \quad b \longrightarrow\left(\left(\Psi_{\mathcal{T}, i}(b)\right)_{i \in \hat{I}}, \operatorname{ev}_{\hat{I}}(b)\right)
$$

is transversal to the submanifold $\{0\} \times \triangle_{V}^{\hat{I}}$. The preimage of this submanifold is precisely the space $\mathcal{M}_{\mathcal{T}}^{(0)}$. Thus, $\mathcal{M}_{\mathcal{T}}^{(0)}$ is a smooth oriented manifold by the Implicit Function Theorem.

Lemma 3.4. For any $k \in[4]$ and $u \in C^{\infty}\left(S^{2} ; V\right), \xi^{(k)}(\infty)=0$. Furthermore,

$$
\begin{gathered}
\tilde{\Psi}((c, r, \theta) \cdot u)=(1+r)\left(\tilde{\Psi} u+c\|d u\|_{2}^{2}\right) \quad \forall(c, r) \in \mathbb{C} \times \mathbb{R} ; \\
\left.\frac{d}{d r} \Psi^{(3)}((0, r, \theta) \cdot u)\right|_{r=0}=\int_{\mathbb{C}}\left|d\left(u \circ q_{N}\right)\right|^{2} \beta^{\prime}(|z|)|z|,
\end{gathered}
$$

where $(c, r) \cdot u$ is defined as in Section 3.1. Finally, $D_{u} \xi_{u}^{(k)}=0$ if $\bar{\partial} u=0$.

Proof. The first and last statements are immediate. We use the change of variables

$$
z \longrightarrow(1+r)^{-1} z-c
$$

to prove $(3.1)$ :

$$
\begin{aligned}
& \int_{\mathbb{C}}\left|d\left(((c, r) \cdot u) \circ q_{N}\right)\right|^{2} z=\int_{\mathbb{C}}(1+r)^{-2}\left|d\left(u \circ q_{N}\right)\right|_{(1+r)^{-1} z-c^{z}}^{2} \\
&=(1+r) \int_{\mathbb{C}}\left|d\left(u \circ q_{N}\right)\right|^{2}(z+c)=(1+r)\left(\tilde{\Psi} u+c\|d u\|_{2}^{2}\right),
\end{aligned}
$$


Similarly,

$$
\begin{aligned}
\left.\frac{d}{d r} \int_{\mathbb{C}}\left|d\left((r \cdot u) \circ q_{N}\right)\right|^{2} \beta(|z|)\right|_{r=0} & =\left.\frac{d}{d r} \int_{\mathbb{C}}\left|d\left(u \circ q_{N}\right)\right|^{2} \beta((1+r)|z|)\right|_{r=0} \\
& =\int_{\mathbb{C}}\left|d\left(u \circ q_{N}\right)\right|^{2} \beta^{\prime}(|z|)|z| .
\end{aligned}
$$

The lemma is now proved, since the action by the $\theta$-component does not change $\tilde{\Psi}$.

If $\mathcal{T}=\left(S^{2}, M, I ; j, \underline{\lambda}\right)$ is a regular bubble type, with notation as above, let

$$
\begin{aligned}
\tilde{\mathcal{K}}_{b} \mathcal{T}=\left\{\sigma=\left(\xi_{I}, w_{M+\hat{I}}\right) \in \mathcal{K}_{b} \mathcal{T}:\right. & \left\langle\sigma, \sigma_{(b, \hat{0})}^{k}\right\rangle=0 \forall k \in[4], \\
& \left.\xi_{i_{1}}(\infty)=\xi_{i_{1}}(\infty) \forall i_{1}, i_{2} \in I-\hat{I}\right\} .
\end{aligned}
$$

By (b) of Definition 3.1 and the same argument as in the proof of Proposition 3.3, we can construct smooth maps $h_{\mathcal{T}, b}^{(1)} \times h_{\mathcal{T}, b}^{(2)}: \tilde{\mathcal{K}}_{b} \mathcal{T}_{\delta(b)} \longrightarrow \Gamma^{\prime}(b) \times(\mathbb{C} \times \mathbb{R})^{I}$ such that each map

$$
\begin{gathered}
H_{\mathcal{T}, b}^{(0)}:\left\{(b, \sigma, \theta) \in \mathcal{K}_{b} \tilde{\mathcal{T}}_{\delta(b)} \times \mathbb{R}^{I}:|\theta|<\pi\right\} \longrightarrow \mathcal{B}_{\mathcal{T}}, \\
H_{\mathcal{T}, b}^{(0)}(\sigma, \theta)=\left(h_{\mathcal{T}, b}^{(2)}(\sigma), \theta\right) \cdot \exp _{b}\left(\sigma+h_{\mathcal{T}, b}^{(1)}(\sigma)\right),
\end{gathered}
$$

is orientation-preserving diffeomorphism onto an open neighborhood of $b$ in $\mathcal{B}_{\mathcal{T}}$.

\subsection{Construction of Nearly Holomorphic Bubble Maps. Let}

$$
\mathcal{T}=(S, M, I ; j, \underline{\lambda})
$$

be a simple bubble type. In this subsection, for all

$$
b \in \mathcal{M}_{\mathcal{T}}^{(0)} \quad \text { and } \quad v=\left(b, v_{\hat{I}}\right)
$$

with $v_{\hat{I}} \in F_{b}^{(0)} \mathcal{T}$ sufficiently small, we construct a bubble map $b(v)$ with domain $\Sigma_{v}$, where $\Sigma_{v}$ is as in Subsection 2.2. The map $u_{b(v)}$ will be just the composite $u_{b} \circ q_{v}$. We then define a Riemannian metric $g_{v, i}$ and a nonnegative function $\rho_{v, i}$ on each component $\Sigma_{v, i}$ of $\Sigma_{v}$. The metrics will be such that the $C^{0}$-norm of the differential of $q_{v}$ is bounded independently of $v_{\hat{I}}$. The nonnegative functions are used to modify the Sobolev norms, in such a way that the norm of the inverse of the operator $D_{b(v)}$ on certain subspaces of $\Gamma(b(v))$ is bounded independently of $v_{\hat{I}}$.

By Proposition 3.3, $\mathcal{M}_{\mathcal{T}}^{(0)}$ is a smooth manifold. If $S=S^{2}$, let

$$
\delta_{\mathcal{T}} \in C^{\infty}\left(\mathcal{M}_{\mathcal{T}}^{(0)} ; \mathbb{R}^{+}\right)
$$

be an $\mathcal{A}(\mathcal{T}) \ltimes \tilde{G}_{\mathcal{T}}$-invariant function such that $\delta_{\mathcal{T}}(b)<r_{T_{b}}$ for all $b \in \mathcal{M}_{\mathcal{T}}^{(0)}$. If $S=\Sigma$, let

$$
\delta_{\mathcal{T}} \in C^{\infty}\left(\mathcal{M}_{\mathcal{T}}^{(0)} ; \mathbb{R}^{+}\right)
$$


be an $\mathcal{A}(\mathcal{T}) \ltimes G_{\mathcal{T}}$-invariant function such that for all

$$
b=(\Sigma, M, I ; x,(j, y), u) \in \mathcal{M}_{\mathcal{T}}^{(0)},
$$

(A1) $4 \delta_{\mathcal{T}}$ is smaller than the function $\delta$ of Lemma 5.1;

(A2) $4 \delta_{\mathcal{T}}(b)<r_{\mathcal{C}_{b}} g_{b, 0}$.

In both cases, it can be assumed that $\delta_{\mathcal{T}}$ does not exceed $\frac{1}{4}$.

If $H$ is a subset of $\hat{I}$, put

$$
\begin{aligned}
F^{(H)} \mathcal{T} & =\left\{v=\left(b, v_{\hat{I}}\right) \in F^{(0)} \mathcal{T}: v_{h}=0 \text { if and if } h \in H\right\}, \\
F^{H} \mathcal{T} & =\left\{v=\left[b, v_{\hat{I}}\right] \in F \mathcal{T}: v_{h}=0 \text { if and if } h \in H\right\} .
\end{aligned}
$$

For any $v=\left(b, v_{\hat{I}}\right) \in F^{(0)} \mathcal{T}$, let $|v|$ denote $|v|_{g_{b}}$ if $S=\Sigma$. From now on, we assume that $\delta \in C^{\infty}\left(\mathcal{M}_{\mathcal{T}}^{(0)} ; \mathbb{R}^{+}\right)$is an $\mathcal{A}(\mathcal{T}) \ltimes G_{\mathcal{T}}$-invariant function if $S=\Sigma$ and an $\mathcal{A}(\mathcal{T}) \ltimes \tilde{G}_{\mathcal{T}}$-invariant function if $S=S^{2}$ such that $8 \delta^{\frac{1}{2}} \leq \delta_{\mathcal{T}}$. If

$$
v=\left(b_{v}, v_{\hat{I}}\right)=\left((S, M, I ; x,(j, y), u), v_{\hat{I}}\right) \in F^{(0)} \mathcal{T}_{\delta},
$$

let $q_{v}: \Sigma_{v} \longrightarrow \Sigma_{b_{v}}$ be the smooth map defined in Subsection 2.2 for

$$
v=\left(\mathcal{C}, v_{\hat{I}}\right)=\left((S, M, I ; x,(j, y)), v_{\hat{I}}\right),
$$

using the metric $g_{b_{v}, \hat{0}}$ on $\Sigma$ if $S=\Sigma$. Let $u_{v}=u_{b_{v}} \circ q_{v}$ and $b(v)=\left(\mathcal{C}(v), u_{v}\right)$.

We now define a Riemannian metric $g_{v, i}$ on $\Sigma_{v, i}$ for each $i \in I(v) \subset I$. Along the way, we construct a metric $g_{v, i}$ on $\Sigma_{b_{v}, i}$ for each $i \in I$. Suppose $i \in I$ and for all $h \in \hat{I}$ such that $\iota_{h}=i$, we have constructed a metric $g_{v, h}$ on $\Sigma_{b_{v}, h}$. For each $h \in \hat{I}$ such that $\iota_{h}=i$ and $v_{h} \neq 0$, let $\tilde{g}_{v, i, h}$ denote the metric on $B_{b_{v}, h}\left(2 \delta\left(b_{v}\right)^{\frac{1}{2}}\right)$ which is the pullback of the metric $g_{v, h}$ by the map

$$
z \longrightarrow q_{N}\left(\frac{\phi_{b_{v}, h}}{v_{h}}\right), \quad \text { where } \quad \phi_{b_{v}, h}= \begin{cases}\phi_{\top_{b_{v}}, h}, & \text { if } x_{h} \in S^{2} \\ \phi_{\top_{b_{v}}, g_{b}, h}, & \text { if } x_{h} \in \Sigma .\end{cases}
$$

This metric is conformal with the original metric $g_{b_{v}, i}$ on $\Sigma_{b_{v}, i}$, because the maps $\phi_{b, h}$ are holomorphic on the set $\left\{r_{b, h} \leq \delta_{\mathcal{T}}(b)\right\}$ and the metric $g_{v, h}$ is conformal with the standard metric on $\mathbb{C}$. Thus, there exists a smooth positive function $\lambda_{v, i, h}$ such that $\tilde{g}_{v, i, h}=\lambda_{v, i, h}^{2} g_{b_{v}, i}$. Let $\lambda_{v, i} \in C^{\infty}\left(\Sigma_{b_{v}, i} ; \mathbb{R}^{+}\right)$ be given by

$$
\lambda_{v, i}(z)= \begin{cases}\lambda_{v, i, h}(z)+\beta_{\left|v_{h}\right|}\left(r_{b_{v}, h}(z)\right)\left(1-\lambda_{v, i, h}(z)\right), & \text { if } \iota_{h}=i, r_{b_{v}, h}(z) \leq 2\left|v_{h}\right|^{\frac{1}{2}} \\ 1, & \text { if } r_{b_{v}, h}(z) \geq 2\left|v_{h}\right|^{\frac{1}{2}} \forall h \in \hat{I} .\end{cases}
$$

Since $I$ is a rooted tree, this procedure defines metrics $g_{v, i}$ for each $i \in I(v)$.

In addition, we define a smooth nonnegative function $\rho_{v, i}$ on $\Sigma_{v, i}$ for each $i \in I(v)$. As in the previous paragraph, along the way we define a function $\rho_{v, i}$ for each $i \in I$. Suppose $i \in I$ and for all $h \in \hat{I}$ such that $\iota_{h}=i$, we have 
constructed a smooth function $\rho_{v, h}$ on $\Sigma_{b_{v}, h}$. Suppose $h \in \hat{I}$ is such that $\iota_{h}=i$ and $z \in \Sigma_{b_{v}, i}$. If $v_{h} \neq 0$ and

$$
|z|_{h} \equiv r_{b_{v}, h}(z)<\delta_{\mathcal{T}}\left(b_{v}\right),
$$

we put

$$
\rho_{v, i}(z)=\rho_{v, h}\left(q_{h, v_{h}} z\right)+\beta\left(\frac{\delta_{\mathcal{T}}\left(b_{v}\right)|z|_{h}}{\left|v_{h}\right|}\right)\left\{\left(|z|_{h}^{2}+\frac{\left|v_{h}\right|^{2}}{|z|_{h}^{2}}\right)-\rho_{v, h}\left(q_{h, v_{h}} z\right)\right\},
$$

where $q_{h, v_{h}}$ is defined as in Section 2.3, using the metric $g_{b_{v}, \hat{0}}$ on $\Sigma$ if $S=\Sigma$. If $v_{h} \neq 0$ and $\delta_{\mathcal{T}}\left(b_{v}\right) \leq|z|_{h} \leq 2 \delta_{\mathcal{T}}\left(b_{v}\right)$, we set

$$
\rho_{v, i}(z)=\left(|z|_{h}^{2}+\frac{\left|v_{h}\right|^{2}}{|z|_{h}^{2}}\right)+\beta\left(\frac{|z|_{h}}{\delta_{\mathcal{T}}\left(b_{v}\right)}\right)\left\{1-\left(|z|_{h}^{2}+\frac{\left|v_{h}\right|^{2}}{|z|_{h}^{2}}\right)\right\} .
$$

If $v_{h}=0$ and $|z|_{h} \leq 2 \delta_{\mathcal{T}}\left(b_{v}\right)$, let

$$
\rho_{v, i}(z)=|z|_{h}^{2}+\beta\left(\frac{|z|_{h}}{\delta_{\mathcal{T}}\left(b_{v}\right)}\right)\left\{1-|z|_{h}^{2}\right\} .
$$

If $|z|_{h} \geq 2 \delta_{\mathcal{T}}\left(b_{v}\right)$ for all $h \in \hat{I}$ with $\iota_{h}=i$ and $v_{i} \neq 0$ if $i>0$, set $\rho_{v, i}(z)=1$. Otherwise, let

$$
\rho_{v, i}(z)=\left|q_{S}^{-1}(z)\right|^{2}+\beta\left(\delta_{\mathcal{T}}\left(b_{v}\right)\left|q_{S}^{-1}(z)\right|\right)\left\{1-\left|q_{S}^{-1}(z)\right|^{2}\right\} .
$$

This construction defines nonnegative functions $\rho_{v, i}$ on $\Sigma_{v, i}$ for all $i \in I(v)$.

We finally define norms on the spaces $\Gamma\left(u_{v}\right)$ and $\Gamma^{1}\left(u_{v}\right)$. If $\eta_{i} \in \Gamma^{1}\left(u_{v, i}\right)$, put

$$
2\left\|\eta_{i}\right\|_{v, p ; i}=\left(\int_{\Sigma_{v, i}}\left|\eta_{i}\right|^{p}\right)^{\frac{1}{p}}+\left(\int_{\Sigma_{v, i}} \rho_{v, i}^{-\frac{p-2}{p}}\left|\eta_{i}\right|^{2}\right)^{\frac{1}{2}}
$$

where $\left|\eta_{i}\right|$ and the integrals are computed with respect to the metric $g_{v, i}$ on $\Sigma_{v, i}$ and $g_{V, b_{v}}$ on $V$. Denote by $\left\|\eta_{i}\right\|_{v, C^{0} ; i}$ the $C^{0}$-norm of $\eta_{i}$ with respect to these metrics. If $\eta=\eta_{I(v)} \in \Gamma^{1}\left(u_{v}\right)$, let

$$
\|\eta\|_{v, p}=\sum_{i \in I(v)}\left\|\eta_{i}\right\|_{v, p ; i}, \quad\|\eta\|_{v, C^{0}}=\sum_{i \in I(v)}\left\|\eta_{i}\right\|_{v, C^{0} ; i}
$$

Similarly, for any $\xi_{i} \in \Gamma\left(u_{v, i}\right)$, put

$$
\begin{aligned}
& 2\left\|\xi_{i}\right\|_{v, p ; i}=\left(\int_{\Sigma_{v, i}}\left|\xi_{i}\right|^{p}\right)^{\frac{1}{p}}+\left(\int_{\Sigma_{v, i}} \rho_{v, i}^{-\frac{p-2}{p}}\left|\xi_{i}\right|^{2}\right)^{\frac{1}{2}} ; \\
& \|\xi\|_{v, p, 1 ; i}=\left\|\xi_{i}\right\|_{v, p ; i}+\left\|\nabla \xi_{i}\right\|_{v, p ; i}
\end{aligned}
$$

where we again use the metrics $g_{v, i}$ on $\Sigma_{v, i}$ and $g_{V, b_{v}}$ on $V$ as in (3.3). Denote by $\left\|\xi_{i}\right\|_{v, C^{0} ; i}$ the $C^{0}$-norm of $\xi_{i}$ with respect to the metric $g_{V, b_{v}}$ on $V$. If 
$\xi=\xi_{I(v)} \in \Gamma\left(u_{v}\right)$, let

$$
\begin{gathered}
\|\xi\|_{v, p}=\sum_{i \in I(v)}\left\|\xi_{i}\right\|_{v, p ; i}, \quad\|\xi\|_{v, p, 1}=\sum_{i \in I(v)}\left\|\xi_{i}\right\|_{v, p, 1 ; i} \\
\|\xi\|_{v, C^{0}}=\sum_{i \in I(v)}\left\|\xi_{i}\right\|_{v, C^{0} ; i}
\end{gathered}
$$

Note that even though the functions $\rho_{v, i}^{-\frac{p-2}{p}}$ have poles at the singular points of $\Sigma_{v}$, all smooth one-forms and vector fields have finite norms defined by (3.3) and (3.4), respectively, since $\frac{p-2}{p}<1$. We denote by $L_{1}^{p}(v)$ the completion of $\Gamma\left(u_{v}\right)$ with respect to the $(v, p, 1)$-norm and by $L^{p}(v)$ the completion of $\Gamma^{0,1}\left(u_{v}\right)$ with respect to the $(v, p)$-norm. Finally, let

$$
D_{v}: \Gamma\left(u_{v}\right) \longrightarrow \Gamma^{0,1}\left(u_{v}\right)
$$

denote the linearization of the $\bar{\partial}$-operator at $u_{v}$ with respect to the metric $g_{V, b_{v}}$ on $V$.

Lemma 3.5. If $\mathcal{T}$ is a simple bubble type and $p>2$, there exist $\delta, C \in$ $C^{\infty}\left(\mathcal{M}_{\mathcal{T}}^{(0)} ; \mathbb{R}^{+}\right)$such that for all $v \in F^{(0)} \mathcal{T}_{\delta}$,

(1) $\left\|d u_{v}\right\|_{v, C^{0}} \leq C\left(b_{v}\right)$ and $\left\|\bar{\partial} u_{v}\right\|_{v, p} \leq C\left(b_{v}\right)|v|^{\frac{1}{p}}$;

(2) $\left\|D_{v} \xi\right\|_{v, p} \leq C\left(b_{v}\right)\|\xi\|_{v, p, 1}$ for all $\xi \in \Gamma\left(u_{v}\right)$;

(3) $\|\xi\|_{v, C^{0}} \leq C\left(b_{v}\right)\|\xi\|_{v, p, 1}$ for all $\xi \in \Gamma\left(u_{v}\right)$;

(4) $\|\xi\|_{v, p, 1} \leq C\left(b_{v}\right)\left(\left\|D_{v} \xi\right\|_{v, p}+\|\xi\|_{v, p}\right)$ for all $\xi \in \Gamma\left(u_{v}\right)$.

Proof. If $h \in I-I(v)$ and $S=S^{2}$, let $A_{v, h}^{ \pm}$be the annulus as in Subsection 2.2. If $S=\Sigma$, let $A_{v, h}^{ \pm}$denote $A_{g_{b_{v}}, v, h}^{ \pm}$. By definition of the norms, $q_{v}$ is an isometry outside of such annuli, and by Lemma 2.2 the $C^{0}$-norm of $d q_{v}$ is bounded on such annuli independently of $v_{\hat{I}}$. Thus, the first part of (1) follows from (2) of Proposition 3.3. Since $\rho_{v} \geq\left|v_{h}\right|$ on $A_{v, h}$, the second part of (1) follows from Lemma 2.2. Statement (2) of the lemma is immediate from the definition of the norms. The last two claims are proved in the appendix; see Propositions 5.7 and 5.11. In fact, the $C^{0}$-norm of $\xi$ is bounded by the usual $L_{1}^{p}$-norm of $\xi$.

3.4. Scale of Variations. In Subsection 3.6, we consider perturbations of the bubble maps $\{b(v)\}$ in directions "away" from the space of such bubble maps. More precisely, we look at replacing $u_{v}$ by $\exp _{b_{v}, u_{v}} \xi$ with $\xi$ lying in a certain subspace of $L_{1}^{p}(v)$ complementary to "the tangent space" of the space of maps $\{b(v)\}$. If $\mathcal{T}$ is regular, one obvious candidate for such a subspace is the $\left(L^{2}, v\right)$-orthogonal complement of the kernel of $D_{v}$. While the construction in Subsection 3.6 would go through, we would run into significant difficulty showing injectivity and surjectivity of the gluing map; see Subsections 4.2 and 4.5. In this subsection, we start by describing a 
choice of the complementary subspace which will work for the purposes of Subsections 3.6, 4.2, and 4.5. We then describe norms on the tangent spaces to $F \mathcal{T}$ and the properties of our setup that are sufficient to show injectivity and surjectivity of the gluing map.

Suppose $v=((S, M, I ; x,(j, y), u), v) \in F^{(0)} \mathcal{T}_{\delta}$, where $\mathcal{T}$ is a simple bubble type as before. For any $\xi \in \Gamma\left(b_{v}\right)$, define $R_{v} \xi \in L_{1}^{p}(v)$ by

$$
\left\{R_{v} \xi\right\}(z)=\xi\left(q_{v}(z)\right) .
$$

Note that $R_{v} \xi$ is smooth outside of the $|I-I(v)|$ circles mapped by $q_{v}$ to the nodes of $\Sigma_{v_{b}}$ and is continuous everywhere, since $\Gamma\left(b_{v}\right)$ is the set of smooth vector fields on the components of $\Sigma_{b_{v}}$ that agree at the nodes. It follows that $R_{v} \xi$ is indeed of class $L_{1}^{p}$. Let $\Gamma_{-}(v)$ be the image of $\operatorname{ker}\left(D_{b_{v}}\right)$ under the map $R_{v}$. This space models the "tangent bundle" to the space of maps $\{b(v)\}$. Denote by $\Gamma_{+}(v)$ its $\left(L^{2}, g_{v}\right)$-orthogonal complement in $L_{1}^{p}(v)$. Let $\pi_{v,-}$ and $\pi_{v,+}$ be the $\left(L^{2}, g_{v}\right)$-orthogonal projections onto $\Gamma_{-}(v)$ and $\Gamma_{+}(v)$, respectively.

With $H \subset \hat{I}$ and $v \in F^{(H)} \mathcal{T}_{\delta}$, let

$$
\begin{gathered}
T_{v} F^{H} \mathcal{T}=\left\{\varpi=\left(\xi, w_{\hat{I} \sqcup M}, \theta_{\hat{I}}, r_{\hat{I}-H}\right):\left(\xi, w_{\hat{I} \sqcup M}\right) \in \mathcal{K}_{b_{v}} \mathcal{T} ; \theta_{h}, r_{h} \in \mathbb{R}\right\} ; \\
\tilde{T}_{v} F^{H} \mathcal{T}=\left\{\left(\xi, w_{\hat{I} \sqcup M}, \theta_{\hat{I}}, r_{\hat{I}-H}\right) \in T_{v} F^{H} \mathcal{T}: w_{h}=0 \forall h \in H\right\} .
\end{gathered}
$$

Given $\varpi$ as above, put

$$
\|\varpi\|=\|\xi\|_{b_{v}, C^{0}}+\sum_{h \in \hat{I}}\left|w_{h}\right|_{b_{v}}+\sum_{l \in M}\left|w_{l}\right|_{b_{v}}+\sum_{h \in \hat{I}}\left|\theta_{h}\right|+\sum_{h \in \hat{I}-H}\left|r_{h}\right| .
$$

If $\delta_{\mathcal{T}}$ and $H_{\mathcal{T}, b_{v}}^{(0)}$ are as in Proposition 3.3 and $\|\varpi\|<\delta_{\mathcal{T}}\left(b_{v}\right)$, put

$$
\begin{aligned}
& b_{\varpi} \equiv(S, M, I ; x(\varpi),(j, y(\varpi)), u(\varpi))=H_{\mathcal{T}, b_{v}}^{(0)}\left(\xi, w_{\hat{I} \sqcup M} ; \theta_{\hat{I}}\right) \in \mathcal{M}_{\mathcal{T}}^{(0)},
\end{aligned}
$$

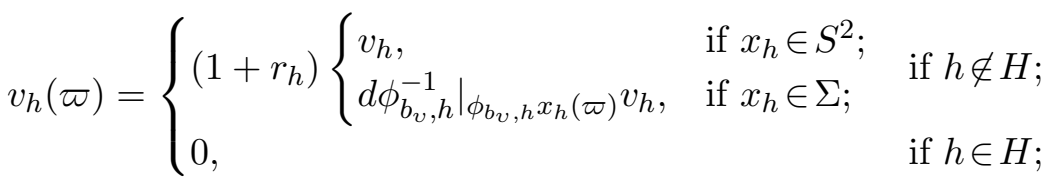

$$
\begin{aligned}
& v(\varpi) \equiv\left(b_{\varpi},(v(\varpi))_{\hat{I}}\right) .
\end{aligned}
$$

Then $v(\varpi) \in F^{(H)} \mathcal{T}_{2 \delta}$ if $\|\varpi\|<\delta\left(b_{v}\right)$ for some $\delta \in C^{\infty}\left(\mathcal{M}_{\mathcal{T}}^{(0)} ; \mathbb{R}^{+}\right)$sufficiently small. If $H=\emptyset, T_{v} F^{H} \mathcal{T}=\tilde{T}_{v} F^{H} \mathcal{T}$ models the tangent space of $[v]$ in $F^{H} \mathcal{T}$. If $H \neq \emptyset$, the bundle $F^{H} \mathcal{T}$ and the construction in the previous subsection lift to a bundle ${ }^{H} F \mathcal{T}$ over

$$
\mathcal{M}_{\mathcal{T}}^{H} \equiv \mathcal{M}_{\mathcal{T}}^{(0)} /\left\{g_{\hat{I}} \in G_{\mathcal{T}}: g_{h}=1 \forall g \in H\right\} .
$$

Then $T_{v} F^{H} \mathcal{T}$ models the tangent space of $[v]$ in ${ }^{H} F \mathcal{T}$. On the other hand, $\tilde{T}_{v} F^{H} \mathcal{T}$ models the tangent space of $[v]$ in the restriction of ${ }^{H} F \mathcal{T}$ to the 
subspace

$$
\left\{\left[b^{\prime}=\left(S, M, I ; x^{\prime},\left(j, y^{\prime}\right), u\right)\right] \in \mathcal{M}_{\mathcal{T}}^{H}: x_{h}^{\prime}=x_{h} \forall h \in H\right\} .
$$

The reason for defining subspaces $\tilde{T}_{v} F^{H} \mathcal{T}$ is that if $x_{h}^{\prime} \neq x_{h}$ for some $h^{\prime} \in H$, $b(v)$ and $b\left(v^{\prime}\right)$ do not have the same singular points for all $v \in F_{b}^{(H)} \mathcal{T}$ and $v \in F_{b^{\prime}}^{(H)} \mathcal{T}$. Since the perturbation construction of Subsection 3.6 does not change the singular points of $b(v)$ and $b\left(v^{\prime}\right)$, the resulting bubble maps $\tilde{b}(v)$ and $\tilde{b}\left(v^{\prime}\right)$ will necessarily be different.

We now define norms on $T_{v} F^{H} \mathcal{T}$, which make the estimates in Lemma 3.6 dependent only on $b_{v}$. If $h \in \hat{I}-H$, let

$$
w_{h}^{\prime}=\phi_{b_{v}, h} q_{v(\varpi), \iota_{h}}\left(q_{v, \iota_{h}}^{-1}\left(\iota_{h}, x_{h}\right)\right) \in F_{h, b_{v}}^{(0)} \quad \text { if } q_{v(\varpi), \iota_{h}}\left(q_{v, \iota_{h}}^{-1}\left(\iota_{h}, x_{h}\right)\right) \in \Sigma_{b_{v}, \iota_{h}} .
$$

In such a case, let

$$
\|\varpi\|_{v, h}=\left|\frac{w_{h}^{\prime}+w_{h}}{v_{h}}\right| .
$$

Otherwise, put $\|\varpi\|_{v, h}=1$. Let $\|\varpi\|_{v}=\|\varpi\|+\sum_{h \in \hat{I}-H}\|\varpi\|_{v, h}$.

In order to simplify notation, we replace $v(\varpi)$ by $\varpi$ whenever there is no ambiguity. If $\|\varpi\|_{v}$ is sufficiently small, define $\zeta_{\varpi} \in \Gamma^{\prime}\left(u_{v}\right)$ by

$$
\exp _{b_{v}, u_{v}} \zeta_{\varpi}=u_{\varpi}, \quad\left\|\zeta_{\varpi}\right\|_{b_{v}, C^{0}}<\operatorname{inj} g_{V, b_{v}} .
$$

Similarly, $l \in M$, define $w_{l}(\varpi) \in T_{y_{l}(v)} \Sigma_{v, j_{l}(v)}$ by

$$
\exp _{g_{v}, y_{l}(v)} w_{l}(\varpi)=y_{l}(v(\varpi)), \quad\left|w_{l}(\varpi)\right| \equiv\left|w_{l}(\varpi)\right|_{g_{v}}<\operatorname{inj}_{y_{l}(v)} g_{v} .
$$

If $\varpi \in \tilde{T} F_{v}^{H} \mathcal{T}$ and $\xi \in \Gamma\left(u_{v}\right)$, let $R_{\varpi} \xi \in \Gamma\left(u_{\varpi}\right)$ be the vector field given by

$$
R_{\varpi} \xi(z)=\Pi_{b_{v}, \zeta_{\varpi}(z)} \xi(z) .
$$

Note that since $b(v)$ and $b(\varpi)$ have the same singular points whenever $\varpi \in$ $\tilde{T} F_{v}^{H} \mathcal{T}, \Pi_{b_{v}, \zeta_{\varpi}}$ does indeed map $\Gamma\left(u_{v}\right)$ to $\Gamma\left(u_{\varpi}\right)$. If $\eta \in \Gamma^{1}\left(u_{v}\right)$, we define $R_{\varpi} \eta \in \Gamma^{1}\left(u_{\varpi}\right)$ similarly. Let $S_{\varpi}$ denote the inverse of $R_{\varpi}$.

Lemma 3.6. There exist $\delta, C \in C^{\infty}\left(\mathcal{M}_{\mathcal{T}}^{(0)} ; \mathbb{R}^{+}\right)$such that for all $v \in F^{(H)} \mathcal{T}_{\delta}$ and $\varpi \in \tilde{T}_{v} F^{H} \mathcal{T}_{\delta}$,

(1) $C\left(b_{v}\right)^{-1}\|\varpi\|_{v} \leq\left\|\zeta_{\varpi}\right\|_{v, p, 1}+\sum_{l \in M}\left|w_{l}(\varpi)\right|_{g_{v}} \leq C\left(b_{v}\right)\|\varpi\|_{v}$;

(2) $\left\|\frac{g_{V, b_{\varpi}}}{g_{V, b_{v}}}-1\right\|_{C^{3}} \leq C\left(b_{v}\right)\|\varpi\|,\left\|\frac{g_{\varpi}}{g_{v}}-1\right\|_{C^{0}} \leq C\left(b_{v}\right)\|\varpi\|_{v}$, and $\| \frac{\rho_{\varpi}}{\rho_{v}}-$ $1\left\|_{C^{0}} \leq C\left(b_{v}\right)\right\| \varpi \|_{v} ;$

(3) $\left\|S_{\varpi} d u_{\varpi}-d u_{v}\right\|_{v, p} \leq C\left(b_{v}\right)\|\varpi\|_{v},\left\|S_{\varpi} \bar{\partial} u_{\varpi}-\bar{\partial} u_{v}\right\|_{v, p} \leq C\left(b_{v}\right)|v|^{\frac{1}{p}}\|\varpi\|_{v}$;

(4) $\left\|S_{\varpi} \nu-\nu\right\|_{v, p} \leq C\left(b_{v}\right)\|\varpi\|_{v}$;

(5) $\left\|S_{\varpi} D_{\varpi} R_{\varpi} \xi-D_{v} \xi\right\|_{v, p} \leq C\left(b_{v}\right)\|\varpi\|_{v}\|\xi\|_{v, p, 1}$ and $\left\|S_{\varpi} \pi_{\varpi, \pm} R_{\varpi} \xi-\pi_{v, \pm} \xi\right\|_{v, p, 1} \leq C\left(b_{v}\right)\|\varpi\|_{v}\|\xi\|_{v, p, 1}$ for all $\xi \in \Gamma\left(u_{v}\right)$. 
Proof. The first statement of (2) is clear. Proofs of (1), the last two claims of (2), (3), and the last claim of (5) are direct, though lengthy, computations, all of the same nature. The statement of (4) is immediate from (1). The first claim of (5) follows from (2) and basic Riemannian geometry estimates as in $[\mathbf{Z 1}]$.

Remark. The second claim in (5) above is proved by choosing an orthonormal basis $\left\{\xi_{b, i}\right\}$ for the kernel of $D_{b}$ for $b$ lying near $b_{v}$ in $\mathcal{M}_{\mathcal{T}}^{(0)}$, so that each $\xi_{b, i}$ varies smoothly with $b$. Then the claim follows immediately from an estimate on $S_{\varpi} R_{v(\varpi)} \xi_{b_{\varpi}, i}-R_{v} \xi_{b, i}$, since the projection maps can be expressed in terms of inner-products with $\xi_{b, i}$. Note that if we had defined $\Gamma_{-}(v)$ to be the kernel of $D_{v}$ in the case $\mathcal{T}$ is regular, this claim, if true, would have been much harder to prove because of the presence of small eigenvalues of $D_{v}^{*} D_{v}$; see Subsection 3.6 for more details.

If $v \in F^{(0)} \mathcal{T}_{\delta},\left(\Sigma_{v}, g_{v}\right)$ can be viewed as a connected sum of the surfaces $\left\{\left(\Sigma_{\mathcal{T}, i}, g_{b_{v}, i}\right)\right\}$ with very thin necks. If $\varpi \in \mathcal{K}_{b_{v}} \mathcal{T} \subset T_{v} F^{\emptyset} \mathcal{T}$ is as above and $\left|w_{h}\right| \geq 2\left|v_{h}\right|^{\frac{1}{2}}$, the maps $u_{v}: \Sigma \longrightarrow V$ and $u_{\varpi}: \Sigma \longrightarrow V$ are very far apart in the $C^{0}$-norm even if $\|\varpi\|$ is small. However, we can still compare the two maps and the various objects of Lemma 3.6, appropriately defined, on the corresponding direct summands. If the gluing map of Subsection 3.6 is defined only on $F^{(\emptyset)} \mathcal{T}_{\delta}$, and not on $F \mathcal{T}_{\delta}$, we need to be able to do such comparisons in order to adjust the gluing map in the presence of constraints $\mu$; see Subsection 3.8.

In order to state an analogue of Lemma 3.6 with $\|\varpi\|_{v}$ for $\varpi \in \tilde{T}_{\varpi} F^{H} \mathcal{T}$ replaced by $\|\varpi\|$ for

$$
\varpi \in \mathcal{K}_{b_{v}} \mathcal{T} \subset \tilde{T}_{\varpi} F^{\emptyset} \mathcal{T}
$$

for each $\varpi \in \mathcal{K}_{b_{v}} \mathcal{T}_{\delta(b)}$, with $\delta$ sufficiently small, we construct a smooth map $\tilde{q}_{\varpi}:\left(\Sigma_{v}, g_{v}\right) \longrightarrow\left(\Sigma_{\varpi}, g_{\varpi}\right)$, which is almost an isometry. The map will depend only on the elements $w_{h} \in F_{b, h}^{(0)}$. The structure of the construction is similar to the construction of the map $q_{v}$ in Subsection 2.2. For each $h \in \hat{I}$ with $\iota_{h}=\hat{0}$, let $\tilde{p}_{h, \varpi}: B_{b_{v}, h}\left(4 \delta_{\mathcal{T}}\left(b_{v}\right)\right) \longrightarrow \Sigma$ be the (holomorphic) $\left(g_{b_{v}, \hat{0}}, g_{b_{\varpi}, \hat{0}}\right)$ isometry provided by Lemma 5.1 . We define $\tilde{q}_{h, \varpi}: \Sigma \longrightarrow \Sigma$ by setting

$$
\tilde{q}_{h, \varpi}(z)=\phi_{b_{v}, h}^{-1}\left\{\phi_{b_{v}, h} \tilde{p}_{h, \varpi}(z)+\beta_{\delta_{\mathcal{T}}^{2}\left(b_{v}\right)}\left(r_{b_{v}, h}(z)\right)\left(\phi_{b_{v}, h}(z)-\phi_{b_{v}, h} \tilde{p}_{h, \varpi}(z)\right)\right\}
$$

if $r_{b_{v}, h}(z) \leq 2 \delta_{\mathcal{T}}\left(b_{v}\right)$ and taking $\tilde{q}_{h, \varpi}(z)=z$ otherwise. If $h \in \hat{I}$ and $\iota_{h} \neq \hat{0}$, define

by setting

$$
\tilde{q}_{h,\left(x_{h}, w_{h}\right)}: \Sigma_{b, \iota_{h}} \longrightarrow \Sigma_{b, \iota_{h}}
$$

$$
\tilde{q}_{h, \varpi}(z)=\phi_{b_{v}, h}^{-1}\left\{\phi_{b_{v}, h}(z)+w_{h}-\beta_{\delta_{\mathcal{T}}^{2}\left(b_{v}\right)}\left(r_{b_{v}, h}(z)\right) w_{h}\right\}
$$


if $r_{b_{v}, h}(z) \leq 2 \delta_{\mathcal{T}}\left(b_{v}\right)$ and taking $\tilde{q}_{h, \varpi}(z)=z$ otherwise. Let $\tilde{q}_{\varpi, \hat{0}}=I d_{\Sigma}$. If $h \in \hat{I}$ and $\tilde{q}_{\varpi, \iota_{h}}: \Sigma \longrightarrow \Sigma$ has been constructed, let

$$
\tilde{q}_{\varpi, h}(z)= \begin{cases}q_{\varpi, \iota_{h}}^{-1}\left(\tilde{p}_{h, \varpi}(z)\left(q_{\varpi, \iota_{h}}\left(\tilde{q}_{\varpi, \iota_{h}}(z)\right)\right)\right), & \text { if } r_{b_{v, h}, h}\left(q_{\varpi, \iota_{h}}(z)\right) \leq 2 \delta_{\mathcal{T}}\left(b_{v}\right) ; \\ \tilde{q}_{\varpi, \iota_{h}}(z), & \text { if } r_{b_{v, h}, h}\left(q_{\varpi, \iota_{h}}(z)\right) \geq 2 \delta_{\mathcal{T}}\left(b_{v}\right) .\end{cases}
$$

Going through all of $I$, we obtain a map $\tilde{q}_{\varpi}: \Sigma \longrightarrow \Sigma$, which shifts the connect-summands of $\left(\Sigma, g_{v}\right)$ to the connect-summands of $\left(\Sigma, g_{\varpi}\right)$. The important properties of such maps $\tilde{q}_{\varpi}$ as summarized below.

Lemma 3.7. There exist $\delta, C \in C^{\infty}\left(\mathcal{M}_{\mathcal{T}}^{(0)} ; \mathbb{R}\right)$ and a smooth family of maps $\left\{\tilde{q}_{\varpi}: \Sigma \longrightarrow \Sigma \mid \varpi \in \mathcal{K}_{b_{v}} \mathcal{T}_{\delta\left(b_{v}\right)} \subset T_{v} F_{v}^{(\emptyset)} \mathcal{T}, v \in F_{b_{v}}^{(\emptyset)} \mathcal{T}_{\delta\left(b_{v}\right)}\right\}, \quad$ such that

(1) $\tilde{q}_{0}=I d_{\Sigma}$ and $q_{v}=q_{\varpi} \circ \tilde{q}_{\varpi}$ on $\Sigma_{b_{v}, i}^{*}=\Sigma_{b_{\varpi}, i}^{*}$ outside of the annuli

$$
A_{\varpi}=\tilde{q}_{\varpi, \iota_{h}}^{-1} q_{\varpi, l_{h}}^{-1}\left(\left\{z \in \Sigma_{b_{v}, \iota_{h}}: \delta_{\mathcal{T}}\left(b_{v}\right) \leq r_{b, h}(z) \leq 2 \delta_{\mathcal{T}}\left(b_{v}\right)\right\}\right),
$$

which contain no marked points of $b(v)$ or $b(\varpi)$.

(2) $\left|\frac{\tilde{q}_{\varpi}^{*} g_{\varpi}}{g_{v}}-\frac{\tilde{q}_{\varpi^{\prime}}^{*} g_{\varpi^{\prime}}}{g_{v}}\right| \leq C\left(b_{v}\right)\left\|\varpi-\varpi^{\prime}\right\|$ for all $\varpi, \varpi^{\prime} \in \mathcal{K}_{b_{v}} \mathcal{T}_{\delta\left(b_{v}\right)}$.

These maps $q_{\varpi}$ allow us to compare operators on vector fields and oneforms on $\left(\Sigma_{v}, u_{v}\right)$ and $\left(\Sigma_{\varpi}, u_{\varpi}\right)$ whenever $\|\varpi\|$ is sufficiently small. Define $\zeta_{\varpi}^{\prime} \in \Gamma\left(u_{v}\right)$ by

$$
\exp _{b_{v}, u_{v}} \zeta_{\varpi}^{\prime}=u_{\varpi} \circ \tilde{q}_{\varpi}, \quad\left\|\zeta_{\varpi}^{\prime}\right\|_{b_{v}, C^{0}} \leq \operatorname{inj} g_{b_{v}} .
$$

For $\xi \in \Gamma\left(u_{v}\right)$, let $R_{\varpi}^{\prime} \xi \in \Gamma\left(u_{\varpi}\right)$ be given by

$$
\left\{R_{\varpi}^{\prime} \xi\right\}(z)=\Pi_{b_{v}, \zeta_{\varpi}^{\prime}\left(\tilde{q}_{\varpi}^{-1}(z)\right)} \xi\left(\tilde{q}_{\varpi}^{-1}(z)\right) .
$$

Similarly, for any $\eta \in \Gamma^{0,1}\left(u_{v}\right)$, let $R_{\varpi}^{\prime} \eta \in \Gamma^{0,1}\left(u_{\varpi}\right)$ be given by

$$
\left.\left\{R_{\varpi}^{\prime} \eta\right\}\right|_{z}=\left.\left.\Pi_{b_{v}, \zeta_{\varpi}^{\prime}\left(\tilde{q}_{\varpi}^{-1}(z)\right)} \circ \eta\right|_{\tilde{q}_{\varpi}^{-1}(z)} \circ \partial \tilde{q}_{\varpi}^{-1}\right|_{z} .
$$

Denote by $S_{\varpi}^{\prime}$ the inverse of $R_{\varpi}^{\prime}$. Similarly to Lemma 3.6, we have

Lemma 3.8. There exist $\delta, C \in C^{\infty}\left(\mathcal{M}_{\mathcal{T}}^{(0)} ; \mathbb{R}^{+}\right)$such that for all $v \in F^{(\emptyset)} \mathcal{T}_{\delta}$ and $\varpi \in \mathcal{K}_{b_{v}} \mathcal{T} \subset \tilde{T}_{v} F^{\emptyset} \mathcal{T}_{\delta}$,

(1) $C\left(b_{v}\right)^{-1}\|\varpi\| \leq\left\|\zeta_{\varpi}^{\prime}\right\|_{v, p, 1}+\sum_{l \in M}\left|w_{l}(\varpi)\right|_{g_{v}} \leq C\left(b_{v}\right)\|\varpi\|$;

(2) $\left\|S_{\varpi}^{\prime} d u_{\varpi}-d u_{v}\right\|_{v, p} \leq C\left(b_{v}\right)\|\varpi\|,\left\|S_{\varpi}^{\prime} \bar{\partial} u_{\varpi}-\bar{\partial} u_{v}\right\|_{v, p} \leq C\left(b_{v}\right)|v|^{\frac{1}{p}}\|\varpi\|$;

(3) $\left\|S_{\varpi}^{\prime} \nu-\nu\right\|_{v, p} \leq C\left(b_{v}\right)\|\varpi\|$;

(4) $\left\|S_{\varpi}^{\prime} D_{\varpi} R_{\varpi}^{\prime} \xi-D_{v} \xi\right\|_{v, p} \leq C\left(b_{v}\right)\|\varpi\|\|\xi\|_{v, p, 1}$ and $\left\|S_{\varpi}^{\prime} \pi_{\varpi, \pm} R_{\varpi}^{\prime} \xi-\pi_{v, \pm} \xi\right\|_{v, p, 1} \leq C\left(b_{v}\right)\|\varpi\|\|\xi\|_{v, p, 1}$ for all $\xi \in \Gamma\left(u_{v}\right)$. 
3.5. Obstruction Bundle Setup. In the next subsection, we look for solutions of the equation

$$
\bar{\partial} \exp _{b_{v}, u_{v}} \xi=t \nu
$$

with $\xi$ lying in a fixed complement of $\Gamma_{-}(v)$. If $t$ is sufficiently small, we are able to solve this equation up to an element of a vector bundle of the same rank as the dimension of $\Gamma_{-}\left(b_{v}\right)$, called obstruction bundle. This element is the obstruction to solving the equation. There are choices to be made for this obstruction bundle as well as for the subspace complementary to $\Gamma_{-}(v)$. We describe in this subsection what conditions these choices must satisfy for the gluing construction to work properly.

$$
\begin{aligned}
& \text { If } b^{*}=\left(S, M, I ; x^{*},\left(j, y^{*}\right), u^{*}\right) \in \mathcal{M}_{\mathcal{T}}^{(0)} \text { and } \\
& \qquad b=\left(S, M, I ; x,(j, y), u_{I}\right)=H_{\mathcal{T}, b^{*}}(\sigma, \theta)
\end{aligned}
$$

for some $\sigma \in \mathcal{K}_{b^{*}} \mathcal{T}$ and $\theta \in \mathbb{R}^{\hat{I}}$, let $\xi_{b^{*}, b}=\xi_{b^{*}, b, I} \in \Gamma^{\prime}(b)$ be given by

$$
\exp _{b^{*}, u_{i}^{*}} \xi_{b^{*}, b, i}=u_{i}, \quad\left\|\xi_{b^{*}, b, i}\right\|_{C^{0}}<\operatorname{inj} g_{V, b^{*}}
$$

Let $\Pi_{b^{*}, b}=\Pi_{b^{*}, \xi_{b^{*}, b}}$.

Definition 3.9. Suppose $b^{*}=\left(S, M, I ; x^{*},\left(j, y^{*}\right), u^{*}\right)$,

$$
b_{k}=\left(S, M, I ; x_{k},\left(j, y_{k}\right), u_{k}\right) \in \mathcal{M}_{\mathcal{T}}^{(0)},
$$

and $v_{k}=\left(b_{k}, v_{k}\right) \in F^{(0)} \mathcal{T}$ are such that the sequences $\left\{b_{k}\right\}$ and $\left\{\left|v_{k}\right|_{b_{k}}\right\}$ converge to $b^{*} \in \mathcal{M}_{\mathcal{T}}^{(0)}$ and $0 \in \mathbb{R}$, respectively.

(a) The sequence $\left\{\xi_{k} \in L_{1}^{p}\left(u_{v_{k}}\right)\right\} C^{0}$-converges to $\xi^{*} \in \Gamma^{\prime}\left(b^{*}\right)$ if

(a-i) the sequence $\left\{\Pi_{b^{*}, b_{k}}^{-1}\left(\xi_{k} \circ q_{v_{k}}^{-1}\right)\right\} C^{0}$-converges to $\xi^{*}$ on compact subsets of $\Sigma_{b^{*}}^{*}$

(a-ii) there exists $C>0$ such that $\left\|\xi_{k}\right\|_{v_{k}, p, 1}<C$ for all $k$.

(b) The sequence of subspaces $\left\{V_{k} \subset \Gamma\left(u_{v_{k}}\right)\right\} C^{0}$-converges to subspace $V^{*} \subset \Gamma\left(b^{*}\right)$ if there exists a sequence of bases $\left\{\left\{\xi_{k, i}\right\}_{i=1}^{i=N} \subset V_{k}\right\}$ such that

(b-i) for each $i$ fixed, the sequence $\left\{\xi_{k, i}\right\} C^{0}$-converges to some $\xi_{i}^{*} \in V^{*}$;

(b-ii) the set $\left\{\xi_{i}^{*}\right\}$ has cardinality $N$ and is a basis for $V^{*}$.

Lemma 3.10. If the sequence $\left\{v_{k}\right\} \subset F^{(0)} \mathcal{T}$ converges to $b^{*} \in \mathcal{M}_{\mathcal{T}}^{(0)}$ and the sequences $\left\{\xi_{k} \in L_{1}^{p}\left(v_{k}\right)\right\}$ and $\left\{\tilde{\xi}_{k} \in L_{1}^{p}\left(v_{k}\right)\right\}$ converge to $\xi^{*} \in \Gamma^{\prime}\left(b^{*}\right)$ and $\tilde{\xi}^{*} \in \Gamma^{\prime}\left(b^{*}\right)$, respectively,

$$
\lim _{k \longrightarrow \infty}\left\langle\left\langle\xi_{k}, \tilde{\xi}_{k}\right\rangle\right\rangle_{v_{k}, 2}=\left\langle\left\langle\xi^{*}, \tilde{\xi}^{*}\right\rangle\right\rangle_{v^{*}, 2}
$$

Proof. If $v_{k} \longrightarrow b^{*}$, the metrics $g_{V, b_{v_{k}}}$ on $V$ and $g_{b_{v_{k}}, i}$ on $\Sigma_{\mathcal{T}, i} C^{0}$-converge to $g_{V, b^{*}}$ and $g_{b^{*}, i}$, respectively. On the other hand, by (a-ii) of Definition 3.9 and (2) of Lemma 3.5, there exists $C>0$ such that

$$
\left\|\xi_{k}\right\|_{v_{k}, C^{0}},\left\|\tilde{\xi}_{k}\right\|_{v_{k}, C^{0}}<C \quad \forall k .
$$


Thus, the claim follows from (a-i) of Definition 3.9.

Definition 3.11. Suppose $\Omega$ is an open subset of $F^{(\emptyset)} \mathcal{T}$ such that $b(v)$ is defined for all $v \in \Omega$. An $\left(\mathcal{A}(\mathcal{T}) \ltimes G_{\mathcal{T}}\right)$-invariant smooth complex subbundle $\tilde{\Gamma}_{-} \longrightarrow \Omega$ of the Banach bundle $L_{1}^{p} \longrightarrow \Omega$ is a tangent-space model over $\Omega$ if

(a) for every sequence $\left\{v_{k}\right\} \subset \Omega$ converging to $b^{*} \in \mathcal{M}_{\mathcal{T}}^{(0)}$, a subsequence of $\left\{\tilde{\Gamma}_{-}\left(v_{k}\right)\right\} C^{0}$-converges to a subspace $V^{*} \subset \Gamma(b)$ such that $\pi_{b,-}: V^{*} \longrightarrow$ $\Gamma_{-}\left(b^{*}\right)$ is an isomorphism;

(b) if $\bar{\pi}_{v,-}: L_{1}^{p}(v) \longrightarrow \tilde{\Gamma}_{-}(v)$ is the $\left(L^{2}, v\right)$-orthogonal projection, there exist $\delta, C \in C^{\infty}\left(\mathcal{M}_{\mathcal{T}}^{(0)} ; \mathbb{R}^{+}\right)$such that for all $v \in \Omega_{\delta}$ and all $\xi \in \Gamma\left(u_{v}\right)$,

(b-i) $\left\|S_{\varpi} \bar{\pi}_{\varpi,-} R_{\varpi} \xi-\bar{\pi}_{v,-} \xi\right\|_{v, 2} \leq C\left(b_{v}\right)\|\varpi\|_{v}\|\xi\|_{v, p, 1}$ for all $\varpi \in$ $T_{v} F^{(\emptyset)} \mathcal{T}_{\delta\left(b_{\nu}\right)}$;

(b-ii) $\left\|S_{\varpi}^{\prime} \bar{\pi}_{\varpi,-} R_{\varpi}^{\prime} \xi-\bar{\pi}_{v,-} \xi\right\|_{v, 2} \leq C\left(b_{v}\right)\|\varpi\|\|\xi\|_{v, p, 1}$ for all $\varpi \in \mathcal{K}_{b_{v}} \mathcal{T} \subset$ $T_{v} F^{(\emptyset)} \mathcal{T}_{\delta\left(b_{\nu}\right)}$

One example of a tangent-space model is $\left\{\Gamma_{-}(v): v \in F^{(\emptyset)} \mathcal{T}_{\delta}\right\}$. In such a case, the limit $V^{*}$ in (a) of Definition 3.11 is $\Gamma_{-}\left(b^{*}\right)$ and thus depends only on $b^{*}$, and not on the sequence $\left\{v_{k}\right\}$. However, for computational reasons, it is sometimes advantageous to work with other choices. With the choices in $[\mathbf{Z 2}]$, the limit $V^{*}$ in (a) of Definition 3.11 usually depends on the sequence.

The following lemma collects some of the implications of (a) of Definition 3.11. Condition (b) is needed in Subsections 4.2 and 4.5. For any tangent space model over $\Omega$ and $v \in \Omega$, we denote the $\left(L^{2}, v\right)$-orthogonal complement of $\tilde{\Gamma}_{-}(v)$ by $\tilde{\Gamma}_{+}(v)$. Write $\tilde{\Gamma}_{+}^{0,1}(v)$ for the image of $\tilde{\Gamma}_{+}(v)$ under the operator $\tilde{D}_{v}$.

Lemma 3.12. Let $\tilde{\Gamma}_{-} \longrightarrow \Omega$ be a tangent-space model. Then there exist $C, \delta \in C^{\infty}\left(\mathcal{M}_{\mathcal{T}}^{(0)} ; \mathbb{R}\right)$ such that for all $v \in \Omega_{\delta}$

(1a) $\|\xi\|_{v, p, 1} \leq C\left(b_{v}\right)\|\xi\|_{v, 2}$ for all $\xi \in \tilde{\Gamma}_{-}(v)$;

(1b) $\left\|\bar{\pi}_{v,-} \xi\right\|_{v, p, 1} \leq C\left(b_{v}\right)\|\xi\|_{v, p, 1}$ for all $\xi \in \Gamma\left(u_{v}\right)$;

(2a) $L_{1}^{p}(v)=\Gamma_{-}(v) \oplus \tilde{\Gamma}_{+}(v)$;

(2b) if $\tilde{\pi}_{-}$and $\tilde{\pi}_{+}$are the projection maps corresponding to the above decomposition,

$$
\left\|\tilde{\pi}_{v, \pm} \xi\right\|_{v, p, 1} \leq C\left(b_{v}\right)\|\xi\|_{v, p, 1} \quad \forall \xi \in \Gamma\left(u_{v}\right) .
$$

Proof. (1) Suppose there exists a sequence $\left\{v_{k} \in \Omega\right\}$ converging to $b^{*} \in \mathcal{M}_{\mathcal{T}}^{(0)}$ and a sequence $\left\{\xi_{k} \in \tilde{\Gamma}_{-}\left(v_{k}\right)\right\}$ such that $\left\|\xi_{k}\right\|_{v_{k}, p, 1}=1$, while $\|\xi\|_{v_{k}, 2} \longrightarrow 0$. Since $\left\|\xi_{k}\right\|_{v_{k}, p, 1}=1$, by (2) of Lemma 3.16 and (a) of Definition 3.11, a subsequence of $\left\{\xi_{k}\right\} C^{0}$-converges to some nonzero $\xi^{*} \in \Gamma\left(b^{*}\right)$. However, since $\left\|\xi_{k}\right\|_{v_{k}, 2} \longrightarrow 0,\left\|\xi^{*}\right\|_{b^{*}, 2}=0$ by Lemma 3.10 . This is a contradiction, 
and thus (1a) holds. Claim (1b) is an immediate consequence of (1a) and (2) of Lemma 3.16.

(2) Claim (2a) is equivalent to saying that no nonzero element of $\tilde{\Gamma}_{-}(v)$ is orthogonal to $\Gamma_{-}(v)$. So, suppose $v_{k} \longrightarrow b^{*} \in \mathcal{M}_{\mathcal{T}}^{(0)}$ and $\left\{\xi_{k} \in \tilde{\Gamma}_{-}\left(v_{k}\right)\right\}$ is such that $\xi_{k}$ is orthogonal to $\Gamma_{-}(v)$ and $\left\|\xi_{k}\right\|_{v_{k}, p, 1}=1$. Since $\xi_{k} \in \tilde{\Gamma}_{-}\left(v_{k}\right)$ and $\left\|\xi_{k}\right\|_{v_{k}, p, 1}=1$, by (a) of Definition 3.11, a subsequence of $\left\{\xi_{k}\right\}$ converges to some nonzero $\xi^{*} \in \Gamma\left(b^{*}\right)$. By Lemma 3.10, $\xi^{*}$ is orthogonal to $\Gamma_{-}(v)$. However, this contradicts the second part of (a) of Definition 3.11.

(3) Due to (1b), Claim (2b) is equivalent to saying that there exist $C, \delta \in$ $C^{\infty}\left(\mathcal{M}_{\mathcal{T}} ; \mathbb{R}\right)$ such that

$$
\|\xi\|_{v, p, 1} \leq C\left(b_{v}\right)\left\|\bar{\pi}_{v,-} \xi\right\|_{v, p, 1} \quad \forall v \in \Omega_{\delta} \text { and } \xi \in \Gamma_{-}(v) .
$$

Suppose there exists a sequence $\left\{v_{k}\right\} \subset \Omega$ converging to some $b^{*} \in \mathcal{M}_{\mathcal{T}}^{(0)}$ and a sequence $\left\{\xi_{k} \in \Gamma_{-}\left(v_{k}\right)\right\}$ such that $\left\|\bar{\pi}_{v_{k},-} \xi_{k}\right\|_{v_{k}, 2} \longrightarrow 0$, while $\left\|\xi_{k}\right\|_{v_{k}, p, 1}=1$. By Definition 3.11, a subsequence of $\left\{\tilde{\Gamma}_{-}\left(v_{k}\right)\right\}$ converges to a subspace $V \subset$ $\Gamma(b)$. On the other hand, a subsequence of $\left\{\xi_{k}\right\} C^{0}$-converges to a nonzero element $\xi^{*} \in \Gamma_{-}\left(b^{*}\right)$, which must be orthogonal to $V$ by Lemma 3.10. This contradicts the second part of (1) of Definition 3.11.

Definition 3.13. Suppose $\Omega$ is an open subset of $F^{(\emptyset)} \mathcal{T}$ such that $b(v)$ is defined for all $v \in \Omega$. An $\left(\mathcal{A}(\mathcal{T}) \ltimes G_{\mathcal{T}}\right)$-invariant smooth complex subbundle $\Gamma_{-}^{0,1}(v) \longrightarrow \Omega$ of the Banach bundle $L^{p} \longrightarrow \Omega$ with the same rank as $\Gamma_{-}^{0,1} \longrightarrow \mathcal{M}_{\mathcal{T}}^{(0)}$ is an obstruction bundle if

(a) there exists $C \in C^{\infty}\left(\mathcal{M}_{\mathcal{T}}^{(0)} ; \mathbb{R}\right)$ such that

$\|\eta\|_{v, p} \leq C\left(b_{v}\right)\|\eta\|_{2} \quad$ and $\quad\left\|D_{v}^{*} \eta\right\|_{v, 1} \leq C\left(b_{v}\right)|v|^{\frac{1}{p}} \quad \forall v \in \Omega, \eta \in \Gamma_{-}^{0,1}(v)$;

(b) if $\pi_{v,-}^{0,1}: L^{p}(v) \longrightarrow \Gamma_{-}^{0,1}(v)$ is the $\left(L^{2}, v\right)$-orthogonal projection, there exists $\delta \in C^{\infty}\left(\mathcal{M}_{\mathcal{T}}^{(0)} ; \mathbb{R}^{+}\right)$such that for all $v \in \Omega_{\delta}$ and all $\eta \in \Gamma^{0,1}\left(u_{v}\right)$,

(b-i) $\left\|S_{\varpi} \pi_{\varpi,-}^{0,1} R_{\varpi} \eta-\pi_{v,-}^{0,1} \eta\right\|_{v, 2} \leq C\left(b_{v}\right)\|\varpi\|_{v}\|\xi\|_{v, p}$ for all $\varpi \in$ $T_{v} F^{(\emptyset)} \mathcal{T}_{\delta\left(b_{\nu}\right)}$

(b-ii) $\left\|S_{\varpi}^{\prime} \pi_{\varpi,-}^{0,1} R_{\varpi}^{\prime} \eta-\pi_{v,-}^{0,1} \eta\right\|_{v, 2} \leq C\left(b_{v}\right)\|\varpi\|\|\xi\|_{v, p}$ for all $\varpi \in \mathcal{K}_{b_{v}} \mathcal{T} \subset$ $T_{v} F^{(\emptyset)} \mathcal{T}_{\delta\left(b_{\nu}\right)}$

Such an obstruction bundle is related to the cokernel bundle $\Gamma_{-}^{0,1} \longrightarrow \mathcal{M}_{\mathcal{T}}^{(0)}$. However, if $\hat{I} \neq \emptyset$, the low eigenspaces of $D_{v} D_{v}^{*}$ are too large to form an obstruction bundle; see Remark below. Examples of bundles that satisfy Definition 3.13 can be found in $[\mathbf{Z 2}]$. Given such an obstruction bundle, we denote by $\pi_{v,+}^{0,1}$ the $\left(L^{2}, v\right)$-orthogonal projection onto $\Gamma_{+}^{0,1}(v)$, the $\left(L^{2}, v\right)$ orthogonal complement of $\Gamma_{-}^{0,1}(v)$. The following lemma is clear from (a) of Definition 3.13. 
Lemma 3.14. If $\Gamma_{-}^{0,1} \longrightarrow \Omega$ is an obstruction bundle, there exists $C \in$ $C^{\infty}\left(\mathcal{M}_{\mathcal{T}} ; \mathbb{R}\right)$ such that

$$
\left\|\pi_{v, \pm}^{0,1} \eta\right\|_{v, p} \leq C\left(b_{v}\right)\|\eta\|_{v, p} \quad \forall v \in \Omega, \eta \in \Gamma^{0,1}\left(u_{v}\right) .
$$

Definition 3.15. If $\mathcal{T}$ is a semiregular bubble type, an obstruction bundle setup for $(V, J, \mathcal{T})$ is a tuple $\left(\delta, \tilde{\Gamma}_{-}, \Gamma_{-}^{0,1}, R\right)$, where

(a) $\delta \in C^{\infty}\left(\mathcal{M}_{\mathcal{T}}^{(0)} ; \mathbb{R}^{+}\right)$is $\left(\mathcal{A}(\mathcal{T}) \ltimes G_{\mathcal{T}}\right)$-invariant and $b(v)$ is defined for all $v \in F^{(0)} \mathcal{T}_{\delta}$

(b) $\tilde{\Gamma}_{-} \longrightarrow F^{(\emptyset)} \mathcal{T}_{\delta}$ and $\Gamma_{-}^{0,1} \longrightarrow F^{(\emptyset)} \mathcal{T}_{\delta}$ are a tangent-space model and an obstruction bundle, respectively;

(c) $R: \pi^{*} \Gamma_{-}^{0,1} \longrightarrow \Gamma_{-}^{0,1}$ is a smooth oriented $\left(\mathcal{A}(\mathcal{T}) \ltimes G_{\mathcal{T}}\right)$-equivariant bundle isomorphism over $F^{(0)} \mathcal{T}_{\delta}$, where $\pi: F^{(0)} \mathcal{T}_{\delta} \longrightarrow \mathcal{M}_{\mathcal{T}}^{(0)}$ is the bundle projection map.

For the rest of the paper, we fix such an obstruction bundle setup. However, whenever we refer to $\delta \in C^{\infty}\left(\mathcal{M}_{\mathcal{T}} ; \mathbb{R}^{+}\right)$, we will mean any function smaller than the function $\delta$ in Definition 3.15. The following lemma states some of the consequences of our setup that are crucial for the construction of the next subsection. If $\mathcal{T}$ is a regular bubble type, we take $\tilde{\Gamma}_{-}(v)$ and $\Gamma_{-}^{0,1}(v)$ to be $\Gamma_{-}(v)$ and $\{0\}$, respectively, and define the other bundles and the projection maps in the same way.

Lemma 3.16. If $\mathcal{T}$ is a simple bubble type, there exist $\delta, C \in C^{\infty}\left(\mathcal{M}_{\mathcal{T}}^{(0)} ; \mathbb{R}^{+}\right)$ such that for any $v \in F^{(0)} \mathcal{T}_{\delta}$ if $\mathcal{T}$ is regular and any $v \in F^{(\emptyset)} \mathcal{T}_{\delta}$ if $\mathcal{T}$ is semiregular,

(1) $\|\xi\|_{v, p, 1} \leq C\left(b_{v}\right)\left\|D_{v} \xi\right\|_{v, p}$ for all $\xi \in \Gamma_{+}(v)$ and all $\xi \in \tilde{\Gamma}_{+}(v)$;

(2) $\left\|\pi_{v,-}^{0,1} \eta\right\|_{v, p} \leq C\left(b_{v}\right)|v|^{\frac{1}{p}}\|\eta\|_{v, p}$ for all $\eta \in \tilde{\Gamma}_{+}^{0,1}(v)$;

(3) $\pi_{v,+}^{0,1}: \tilde{\Gamma}_{+}^{0,1}(v) \longrightarrow \Gamma_{+}^{0,1}(v)$ is an isomorphism with the norm of the inverse bounded by $C\left(b_{v}\right)$.

Proof. (1) The first statement of the lemma is proved in the appendix; see Proposition 5.13. It is consequence of (2) and (4) of Lemma 3.5 and of (a) of Definition 3.11. The second claim is immediate from (a) of Definition 3.13 and the first claim.

(2) Let $W$ be the $\left(L^{2}, g_{v}\right)$-orthogonal complement of $\pi_{v,+}^{0,1}\left(\tilde{\Gamma}_{+}^{0,1}(v)\right)$ in $\Gamma_{+}^{0,1}(v)$. The second claim implies that

$$
L^{p}(v)=\left(\Gamma_{-}^{0,1}(v) \oplus W\right) \oplus \tilde{\Gamma}_{+}^{0,1}(v) .
$$


Since $\tilde{\Gamma}_{+}^{0,1}(v)$ is the image of $\tilde{\Gamma}_{+}(v)$ under $D_{v}$, with respect to the decompositions $(3.5)$ and $L_{1}^{p}(v)=\Gamma_{-}(v) \oplus \tilde{\Gamma}_{+}(v)$,

$$
D_{v}=\left|\begin{array}{cc}
D_{v}^{(--)} & 0 \\
D_{v}^{(+-)} & D_{v}^{(++)}
\end{array}\right| .
$$

Since $D_{v}^{(++)}$is an isomorphism by (1) of the lemma,

(3.6) ind $D_{v}=$ ind $D_{v}^{(--)}=\operatorname{dim} \Gamma_{-}(v)-\left(\operatorname{dim} \Gamma_{-}^{0,1}(v)+\operatorname{dim} W\right)$

$$
=\left(\operatorname{dim} \Gamma_{-}\left(b_{v}\right)-\operatorname{dim} \Gamma_{-}^{0,1}\left(b_{v}\right)\right)+\operatorname{dim} W=\operatorname{ind} D_{b_{v}}-\operatorname{dim} W .
$$

On the other hand, by the Index Theorem, with $n=\operatorname{dim}_{\mathbb{C}} V$,

$$
\text { ind } \begin{aligned}
D_{v} & =2\left(\sum_{h \in \hat{I}(v)}\left(\left\langle c_{1}(V, J), \lambda_{i}(v)\right\rangle-n\left(g\left(\Sigma_{\mathcal{T}, i}\right)-1\right)\right)-n(|\hat{I}(v)|-1)\right) \\
& =2\left(\sum_{h \in \hat{I}(v)}\left\langle c_{1}(V, J), \lambda_{i}\right\rangle-n(g(S)-1)\right)=\text { ind } D_{b_{v}} .
\end{aligned}
$$

By equations (3.6) and (3.7), $W=\{0\}$, and the last claim of the lemma follows from the second one.

Remark. It is essential for claim (1) of Lemma 3.16 that $p>2$. The operator $D_{v}^{*} D_{v}$ has at least $|\hat{I}|(\operatorname{dim} V)$ eigenvalues that tend to 0 as $|v| \longrightarrow 0$. The corresponding eigenfunctions converge to vector fields on the components of $\Sigma_{b}$ that do not agree at the nodes. If $\mathcal{T}$ is semiregular, the operator $D_{b_{v}}$ has cokernel $\Gamma_{-}^{0,1}(b)$. In such a case, the number of low eigenvalues of $D_{v}^{*} D_{v}$, including 0 , is $\left(\operatorname{dim} \Gamma_{-}^{0,1}(b)\right)+|\hat{I}|(\operatorname{dim} V)$.

Let $\tilde{\pi}_{v,+}^{0,1}: \Gamma_{+}^{0,1}(v) \longrightarrow \tilde{\Gamma}_{+}^{0,1}(v)$ denote the inverse of $\pi_{v,+}^{0,1}: \tilde{\Gamma}_{+}^{0,1}(v) \longrightarrow$ $\Gamma_{+}^{0,1}(v)$. We extend $\tilde{\pi}_{v,+}^{0,1}$ to all of $L^{p}(v)$ by taking it to be $\tilde{\pi}_{v,+}^{0,1} \circ \pi_{v,+}^{0,1}$. If $\eta \in \tilde{\Gamma}_{+}^{0,1}(v)$, let $P_{v} \eta \in \tilde{\Gamma}_{+}(v)$ be the unique element such that $D_{v} P_{v} \eta=\eta$. We extend $P_{v}$ to all of $L^{p}(v)$ by taking it to be $P_{v} \circ \tilde{\pi}_{v,+}^{0,1}$. From Lemma 3.16, we immediately obtain

Corollary 3.17. If $\mathcal{T}$ is a simple bubble type, there exist $\delta, C \in$ $C^{\infty}\left(\mathcal{M}_{\mathcal{T}}^{(0)} ; \mathbb{R}^{+}\right)$such that for all $v \in F^{(0)} \mathcal{T}_{\delta}$ if $\mathcal{T}$ is regular and $v \in F^{(\emptyset)} \mathcal{T}_{\delta}$ if $\mathcal{T}$ is semiregular,

(1) $\left\|\tilde{\pi}_{v,+}^{0,1} \eta\right\|_{v, p} \leq C\left(b_{v}\right)\|\eta\|_{v, p}$ for all $\eta \in \Gamma^{0,1}(v)$;

(2) $\left\|P_{v} \eta\right\|_{v, p, 1} \leq C\left(b_{v}\right)\|\eta\|_{v, p}$ for all $\eta \in \Gamma^{0,1}(v)$. 
3.6. The Gluing Map. In this subsection, we look for small vector fields $\xi \in \tilde{\Gamma}_{+}(v)$ such that $\exp _{b_{v}, u_{v}} \xi$ is holomorphic if $\mathcal{T}$ is regular and lies in $\mathcal{M}_{\Sigma, t \nu, \lambda}$ if $\mathcal{T}$ is semiregular. In Subsection 4.5, we show that all holomorphic maps if $\mathcal{T}$ is regular and all maps in $\mathcal{M}_{\Sigma, t \nu, \lambda} \times \Sigma^{M}$ if $\mathcal{T}$ is semiregular that lie near $\mathcal{M}_{\mathcal{T}}$ with respect to the Gromov topology can be obtained in this way.

If $\xi \in \Gamma\left(u_{v}\right)$, define $\exp _{v} \xi: \Sigma_{v} \longrightarrow V$ and $\bar{\partial}_{v} \xi \in \Gamma^{0,1}\left(u_{v}\right)$ by

$$
\left\{\exp _{v} \xi\right\}(z)=\exp _{b_{v}, u_{v}(z)} \xi(z),\left.\quad\left\{\bar{\partial}_{v} \xi\right\}\right|_{z}=\left.\Pi_{b_{v}, \xi(z)}^{-1} \circ \bar{\partial}\left\{\exp _{v} \xi\right\}\right|_{z} .
$$

If $S=\Sigma$ and $\nu \in \Gamma\left(\Sigma ; \Lambda^{0,1} \pi_{\Sigma}^{*} T^{*} \Sigma \otimes \pi_{V}^{*} T V\right)$, let $\nu_{v, \xi} \in \Gamma^{0,1}\left(u_{v}\right)$ be given by

$$
\left.\nu_{v, \xi}\right|_{z}=\left.\Pi_{b_{v}, \xi(z)}^{-1} \circ \nu\right|_{\left(z,\left\{\exp _{v} \xi\right\} z\right)} .
$$

Then,

$$
\bar{\partial}\left\{\exp _{v} \xi\right\}(\cdot)=\left.t \nu\right|_{\left(\cdot,\left\{\exp _{v} \xi\right\}(\cdot)\right)} \Longleftrightarrow \bar{\partial}_{v} \xi=t \nu_{v, \xi}
$$

Write

$$
\bar{\partial}_{v} \xi=\bar{\partial} u_{v}+D_{v} \xi+N_{v} \xi \quad \text { and }\left.\quad \nu_{v, \xi}\right|_{z}=\left.\nu\right|_{\left(z, u_{v}(z)\right)}+\left.L_{\nu, v} \xi\right|_{z}
$$

Then the second equation in (3.8) is equivalent to

$$
D_{v} \xi+N_{v, t \nu} \xi=t \nu-\bar{\partial} u_{v}
$$

and by Proposition 2.11 in $[\mathbf{Z 1}]$ and (1) of Lemma 3.5, there exist $C_{\bar{\partial}}, \delta \in$ $C^{\infty}\left(\mathcal{M}_{\mathcal{T}}^{(0)} ; \mathbb{R}^{+}\right)$such that for any $v \in F^{(0)} \mathcal{T}_{\delta}$ and $\xi_{1}, \xi_{2} \in \Gamma\left(u_{v}\right)$,

$$
\left\|N_{v, t \nu} \xi_{1}-N_{v, t \nu} \xi_{2}\right\|_{v, p} \leq C_{\bar{\partial}}\left(b_{v}\right)\left(\left\|\xi_{1}\right\|_{v, p, 1}+\left\|\xi_{2}\right\|_{v, p, 1}+t\right)\left\|\xi_{1}-\xi_{2}\right\|_{v, p, 1} .
$$

If $\mathcal{T}$ is semiregular, the term $\nu$ will be fixed, and we will be looking for solutions of (3.10) with $t>0$ very small for $v \in F^{(\emptyset)} \mathcal{T}_{\delta}$. If $\mathcal{T}$ is regular, we will consider (3.10) with $t=0$ and $v \in F^{(0)} \mathcal{T}_{\delta}$. In both cases, we will consider only solutions $\xi$ of (3.10) that lie in the subspace $\tilde{\Gamma}_{+}(v)$ of $L_{1}^{p}(v)$, since the subspace $\Gamma_{-}(v)$ corresponds to moving along the image of the pregluing $\operatorname{map} v \longrightarrow b(v)$.

Vector field $\xi=P_{v} \eta$ with $\eta \in \Gamma_{+}^{0,1}(v)$ solves equation (3.10) if and only if

$$
\begin{gathered}
\eta+\pi_{v,+}^{0,1} N_{v, t \nu} P_{v} \eta=\pi_{v,+}^{0,1}\left(t \nu-\bar{\partial} u_{v}\right) \\
\text { and } \quad \pi_{v,-}^{0,1}\left(t \nu-\bar{\partial} u_{v}-\tilde{\pi}_{v,+}^{0,1} \eta-N_{v, t \nu} P_{v} \eta\right)=0 .
\end{gathered}
$$

Denote the map $\eta \longrightarrow \pi_{v,+}^{0,1} N_{v, t \nu} P_{v} \eta$ by $N_{v, t \nu}^{+}$. By Corollary 3.17 and equation $(3.11)$, there exist $\tilde{C}_{\bar{\partial}}, \delta \in C^{\infty}\left(\mathcal{M}_{\mathcal{T}}^{(0)} ; \mathbb{R}^{+}\right)$such that for any $v \in F^{(0)} \mathcal{T}_{\delta}$ if $\mathcal{T}$ is regular and $v \in F^{(\emptyset)} \mathcal{T}_{\delta}$ if $\mathcal{T}$ is semiregular,

$$
\left\|N_{v, t \nu}^{+} \eta_{1}-N_{v, t \nu}^{+} \eta_{2}\right\|_{v, p} \leq \tilde{C}_{\bar{\partial}}\left(b_{v}\right)\left(\left\|\eta_{1}\right\|_{v, p}+\left\|\eta_{2}\right\|_{v, p}+t\right)\left\|\eta_{1}-\eta_{2}\right\|_{v, p}
$$

for all $\eta_{1}, \eta_{2} \in \Gamma_{+}^{0,1}(v)$ such that $\left\|\eta_{1}\right\|_{v, p},\left\|\eta_{2}\right\|_{v, p} \leq \delta(b)$. 
Lemma 3.18. There exist $\epsilon, \delta \in C^{\infty}\left(\mathcal{M}_{\mathcal{T}}^{(0)} ; \mathbb{R}^{+}\right)$such that for all $v \in F^{(0)} \mathcal{T}_{\delta}$ and $t=0$ if $\mathcal{T}$ is regular, $v \in F^{(\emptyset)} \mathcal{T}_{\delta}$ and $t \in\left[0 ; \delta\left(b_{v}\right)\right]$ if $\mathcal{T}$ is semiregular, and $\alpha \in \Gamma_{+}^{0,1}(v)$ with $\|\alpha\|_{v, p}<\epsilon\left(b_{v}\right)$, the equation

$$
\eta+N_{v, t \nu}^{+} \eta=\alpha
$$

has a unique solution $\eta_{\alpha}$ in $\Gamma_{+}^{0,1}(v)$ such that $\left\|\eta_{\alpha}\right\|_{v, p} \leq 2 \epsilon\left(b_{v}\right)$. Furthermore, such a solution satisfies $\left\|\eta_{\alpha}\right\|_{v, p} \leq 2\|\alpha\|_{v, p}$.

Proof. Put $\epsilon(b)=\left(6 \tilde{C}_{\bar{\partial}}(b)\right)^{-1}$, where $\tilde{C}_{\bar{\partial}}$ is as in (3.14). Define

$$
\Psi_{\alpha}:\left\{\eta \in \Gamma_{+}^{0,1}(v):\|\eta\|_{v, p} \leq 2\|\alpha\|_{v, p}\right\} \longrightarrow \Gamma_{+}^{0,1}(v)
$$

by $\Psi_{\alpha}(\eta)=\alpha-N_{v, t \nu}^{+} \eta$. By equation (3.14),

$$
\begin{aligned}
\left\|\Psi_{\alpha}(\eta)\right\|_{v, p} \leq\|\alpha\|_{v, p}+\tilde{C}_{\bar{\partial}}\left(b_{v}\right)\left(\|\eta\|_{v, p}+t\right)\|\eta\|_{v, p} \leq 2\|\alpha\|_{v, p} & \\
\left\|\Psi_{\alpha}\left(\eta_{1}\right)-\Psi_{\alpha}\left(\eta_{2}\right)\right\|_{v, p} & \leq \tilde{C}_{\bar{\partial}}\left(b_{v}\right)\left(\left\|\eta_{1}\right\|_{v, p}+\left\|\eta_{2}\right\|_{v, p}+t\right)\left\|\eta_{1}-\eta_{2}\right\|_{v, p} \\
& \leq \frac{5}{6}\left\|\eta_{1}-\eta_{2}\right\|_{v, p} .
\end{aligned}
$$

It follows that $\Psi_{\alpha}$ is a contracting operator, and thus has a unique fixed point $\eta_{\alpha}$, i.e.,

$$
\eta_{\alpha}+N_{v, t \nu}^{+} \eta_{\alpha}=\alpha, \quad \text { and } \quad\left\|\eta_{\alpha}\right\|_{v, p} \leq 2\|\alpha\|_{v, p} .
$$

The uniqueness claim follows immediately by taking the difference of the corresponding equations.

Corollary 3.19. If $\mathcal{T}$ is a simple bubble type, there exist $\mathbb{R}^{+}$-valued smooth functions $\delta, \epsilon, C$ on $\mathcal{M}_{\mathcal{T}}^{(0)}$ such that for all $v \in F^{(0)} \mathcal{T}_{\delta}$ and $t=0$ if $\mathcal{T}$ is regular and $v \in F^{(\emptyset)} \mathcal{T}_{\delta}$ and $t \in\left[0 ; \delta\left(b_{v}\right)\right]$ if $\mathcal{T}$ is semiregular, there exists a unique $\eta_{v, t \nu} \in \Gamma^{0,1}(v)$ such that $\eta_{v, t \nu}$ satisfies equation (3.12) and $\left\|\eta_{v, t \nu}\right\|_{v, p} \leq \epsilon\left(b_{v}\right)$. Furthermore,

$$
\left\|\eta_{v, t \nu}\right\|_{v, p} \leq C\left(b_{v}\right)\left(t+|v|^{\frac{1}{p}}\right)
$$

Proof. This corollary follows from Lemmas 3.18 and 3.5.

We now put $\xi_{v, t \nu}=P_{v} \eta_{v, t \nu}$ and $\tilde{u}_{v, t \nu}=\exp _{v} \xi_{v, t \nu}$. Replacing $u_{v}$ in $b(v)$ by $\tilde{u}_{v, t \nu}$, we obtain a new bubble map that will be called $\tilde{b}_{t \nu}(v)$. If $\mathcal{T}$ is regular (and thus $t=0$ ), we will write $\tilde{u}_{v}$ and $\tilde{b}(v)$ for $\tilde{u}_{v, 0}$ and $\tilde{b}_{0}(v)$, respectively. We can assume that the functions $\delta, \epsilon$ and $C$ of Corollary 3.19 are $\left(\mathcal{A}(\mathcal{T}) \ltimes \tilde{G}_{\mathcal{T}}\right)$-invariant if $S=S^{2}$ and $\left(\mathcal{A}(\mathcal{T}) \ltimes G_{\mathcal{T}}\right)$-invariant if $S=\Sigma$. For $\mathcal{T}$ regular, we have thus constructed a gluing map

$$
\tilde{\gamma}_{\mathcal{T}}^{(0)}: F^{(0)} \mathcal{T}_{\delta} \longrightarrow \overline{\mathcal{M}}_{\langle\mathcal{T}\rangle}, \quad v \longrightarrow \tilde{b}(v) .
$$

Since this map is $\left(\mathcal{A}(\mathcal{T}) \ltimes G_{\mathcal{T}}\right)$-invariant, as can be seen from the construction, $\tilde{\gamma}_{\mathcal{T}}^{(0)}$ induces a map on the quotient

$$
\tilde{\gamma}_{\mathcal{T}}: F \mathcal{T}_{\delta} \longrightarrow \overline{\mathcal{M}}_{\langle\mathcal{T}\rangle}
$$


By the smooth dependence of solutions of (3.12), the restrictions

$$
\tilde{\gamma}_{\mathcal{T}}^{(0)}: F^{(H)} \mathcal{T}_{\delta} \longrightarrow \mathcal{M}_{\mathcal{T}(H)}^{(0)}
$$

are smooth. However, continuity of $\tilde{\gamma}_{\mathcal{T}}$ on all of $F \mathcal{T}_{\delta}$ is not immediate. In the next section, we show the map $\tilde{\gamma}_{\mathcal{T}}$ is a homeomorphism onto a neighborhood of $\mathcal{M}_{\mathcal{T}}$ in $\overline{\mathcal{M}}_{\langle\mathcal{T}\rangle}$.

If $\mathcal{T}$ is semiregular and $t>0$, we have constructed a map

$$
\tilde{\gamma}_{\mathcal{T}, t \nu}^{(0)}:\left.F^{(\emptyset)} \mathcal{T}_{\delta}\right|_{\epsilon^{-1}(-t, t)} \longrightarrow C_{(\lambda ; M)}^{\infty}(\Sigma ; V),
$$

which again is $\left(\mathcal{A}(\mathcal{T}) \ltimes G_{\mathcal{T}}\right)$-invariant and thus descends to a map

$$
\tilde{\gamma}_{\mathcal{T}, t \nu}:\left.F^{\emptyset} \mathcal{T}_{\delta}\right|_{\epsilon^{-1}(-t, t) /\left(\mathcal{A}(\mathcal{T}) \ltimes G_{\mathcal{T}}\right)} \longrightarrow C_{(\lambda ; M)}^{\infty}(\Sigma ; V) .
$$

The map $u_{\tilde{b}_{t \nu}(v)}$ lies in $\mathcal{M}_{\Sigma, t \nu, \lambda}$ if and only if equation (3.13) is satisfied, i.e.,

$$
R_{v} \psi_{\mathcal{T}, t \nu}(v) \equiv t \nu-\bar{\partial} u_{v}-\tilde{\pi}_{v,+}^{0,1} \eta_{v, t \nu}-N_{v, t \nu} P_{v} \eta_{v, t \nu}=0 \in \Gamma_{-}^{0,1}(v)
$$

since $\eta_{v, t \nu}$ satisfies equation (3.12).

3.7. An Implicit Function Theorem. In this subsection, we prove a refined version of the Implicit Function Theorem. It will be used in the rest of this section to modify the gluing maps of Subsection 3.6 for the spaces $\mathcal{M}_{\mathcal{T}}(\mu), \mathcal{U}_{\mathcal{T}}(\mu)$, etc.

Let $\mathcal{S}$ be a smooth oriented manifold, and $\mathcal{N} S, \mathcal{N}^{\mu}$, and $F$ oriented Riemannian vector bundles over $\mathcal{S}$. We denote by $b,(b, \vec{n}),(b, \sigma)$, and $(b, v)$ general elements of $\mathcal{S}, \mathcal{N} S, \mathcal{N}^{\mu}$, and $F$, respectively. If $\Omega$ is any subset of $F$ and $\delta>0$, let

$$
\Omega(\delta)=\{(b, \vec{n}, v) \in \mathcal{N} S \oplus F:(b, v) \in \Omega ;|\vec{n}|,|v|<\delta\} .
$$

Let $U$ be an open neighborhood of $\mathcal{S}$ in $\mathcal{N} S \oplus \mathcal{N}^{\mu} \oplus F$ and $h: U \longrightarrow \mathbb{R}^{k}$ a smooth map such that

$$
\begin{gathered}
h(b, \vec{n}, \sigma, v)=h(b, \vec{n}, \sigma, 0),\left.\quad h\right|_{\mathcal{S}}=0, \quad \text { and } \\
d\left(h: \mathcal{N}_{b}^{\mu} \longrightarrow \mathbb{R}^{n}\right)_{(b, 0)}: \mathcal{N}_{b}^{\mu} \longrightarrow \mathbb{R}^{k}
\end{gathered}
$$

is an orientation-preserving isomorphism for all $b \in \mathcal{S}$. Let $\tilde{U}$ be a subset of $U$ such that $\tilde{U}$ is the fiber product along $\mathcal{S}$ of an open neighborhood of $\mathcal{S}$ in $\mathcal{N} S \oplus \mathcal{N}^{\mu}$ and an open subset $\Omega$ of $F$. Suppose $\delta_{\mathcal{S}}>0, C \in C^{\infty}\left(\mathcal{S} ; \mathbb{R}^{+}\right)$, and $\tilde{h}_{t}: \tilde{U} \longrightarrow \mathbb{R}^{k}$ is a family of smooth functions with $t \in\left[0, \delta_{\mathcal{S}}\right]$ such that

$$
\begin{aligned}
&\left|\tilde{h}_{t}-h\right|_{(b, \vec{n}, \sigma, v)},\left|\frac{\partial \tilde{h}_{t}}{\partial \sigma}-\frac{\partial h}{\partial \sigma}\right|_{(b, \vec{n}, \sigma, v)} \leq C(b)\left(|v|^{\frac{1}{p}}+t\right) \\
& \forall t \in\left(0, \delta_{\mathcal{S}}\right),(b, \vec{n}, \sigma, v) \in \tilde{U}
\end{aligned}
$$

where $\frac{\partial h}{\partial \sigma}$ denotes the differential of $h$ along the fibers of $\mathcal{N}^{\mu}$. 
Lemma 3.20. Let $B$ be an open ball about $0 \in \mathbb{R}^{k}$. If $f: B \longrightarrow \mathbb{R}^{k}$ is a smooth function and

$$
k|D f|_{z}-\left.\left.D f\right|_{0}|<|\left(\left.D f\right|_{0}\right)^{-1}\right|^{-1} \quad \forall z \in B,
$$

then $f$ is injective on $B$.

Proof. Let $f_{i}$ denote the $i$ th component of $f$. By the Mean Value Theorem, for all $x, y \in B$, there exists $z_{i}(x, y) \in B$ such that

$$
\left|f_{i}(x)-f_{i}(y)\right|=\left|D f_{i}\right|_{z_{i}(x, y)}|| x-y \mid .
$$

Adding up these equations over all $i$, we obtain

$$
\begin{aligned}
\sum_{i=1}^{i=k}\left|f_{i}(x)-f_{i}(y)\right| & \geq \sum_{i=1}^{i=k}\left|D f_{i}\right|_{0}|| x-\left.y\left|-k \sup _{z \in B}\right| D f\right|_{z}-\left.D f\right|_{0}|| x-y \mid \\
& \geq\left(\left|\left(\left.D f\right|_{0}\right)^{-1}\right|^{-1}-k \sup _{z \in B}|D f|_{z}-\left.D f\right|_{0} \mid\right)|x-y| .
\end{aligned}
$$

Lemma 3.21. For every precompact subset $K$ of $\mathcal{S}$, there exists $\epsilon>0$ such that for all $t \in(0, \epsilon)$ and $\left.(b, \vec{n}, v) \in \Omega(\epsilon)\right|_{K}$, the map

$$
\left\{(b, \sigma) \in \mathcal{N}^{\mu}:|\sigma|<\epsilon\right\} \longrightarrow \tilde{h}_{t}(b, \vec{n}, \sigma, v)
$$

is defined and injective, and its differential defines an orientation-preserving isomorphism between $\mathcal{N}_{b}^{\mu}$ and $\mathbb{R}^{k}$.

Proof. The map above is defined as long as

$$
\left\{(b, \vec{n}, \sigma, v) \in \mathcal{N} S \oplus \mathcal{N}_{b}^{\mu} \oplus F: b \in K,(b, \vec{n}, v) \in \Omega(\epsilon),|\sigma|<\epsilon\right\} \subset \tilde{U} .
$$

Since $K$ is precompact, existence of $\delta>0$ such that the last inclusion holds is trivial. The other two statements follow from the third property of $h$ and the second property of $\tilde{h}_{t}$ (see above); Lemma 3.20 is needed to prove the injectivity. Note that the variation of $\frac{\partial \tilde{h}_{t}}{\partial \sigma}$ over $K$ can be bounded from the variation $\frac{\partial h}{\partial \sigma}$ and the second property of $\tilde{h}_{t}$.

Lemma 3.22. For every precompact subset $K$ of $\mathcal{S}$ and $\epsilon>0$ sufficiently small, there exists $\delta>0$ such that for all $t \in(0, \delta)$ and $\left.(b, \vec{n}, v) \in \Omega(\delta)\right|_{K}$, the image of the map

$$
\left\{(b, \sigma) \in \mathcal{N}^{\mu}:|\sigma|<\epsilon\right\} \longrightarrow \tilde{h}_{t}(b, \vec{n}, \sigma, v)
$$

contains $0 \in \mathbb{R}^{k}$.

Proof. We assume $\epsilon>0$ does not exceed the number provided by Lemma 3.21. Then by precompactness of $K$ and the proof of Lemma 3.21,

$$
\varepsilon \equiv \min \left\{|h(b, \vec{n}, \sigma, v)|:(b, \vec{n}, v) \in \Omega_{\epsilon}\left|K,(b, \sigma) \in \mathcal{N}^{\mu},\right| \sigma \mid=\frac{1}{2} \epsilon\right\}>0 .
$$


Since for each $\left.(b, \vec{n}, v) \in \Omega(\epsilon)\right|_{K}$, the image of the map

$$
\left\{(b, \sigma) \in \mathcal{N}^{\mu}:|\sigma|<\epsilon\right\} \longrightarrow h(b, \vec{n}, \sigma, v)
$$

contains a neighborhood of 0 in $\mathbb{R}^{k}$ and $\tilde{h}_{t}$ is continuous, the claim follows from the first property of $\tilde{h}_{t}$ along with equation (3.18).

Corollary 3.23. For every precompact open subset $K$ of $\mathcal{S}$, there exist $\delta, C>0$ with the following property. For all $t \in(0, \delta)$, there exists a smooth section

$$
\varphi_{t} \in \Gamma\left(\left.\Omega(\delta)\right|_{K} ; \pi^{*} \mathcal{N}^{\mu}\right),
$$

where $\pi:\left.\Omega(\delta)\right|_{K} \longrightarrow K$ is the bundle projection map, such that

$$
\left.\Omega(\delta)\right|_{K} \longrightarrow \tilde{h}_{t}^{-1}(0), \quad(b, \vec{n}, v) \longrightarrow\left(b, \vec{n}, \varphi_{t}(b, \vec{n}, v), v\right),
$$

is an orientation-preserving diffeomorphism. Furthermore,

$$
\left|\varphi_{t}(b, \vec{n}, v)\right| \leq\left. C\left(|v|^{\frac{1}{p}}+t+|\vec{n}|\right) \quad \forall(b, \vec{n}, v) \in \Omega(\delta)\right|_{K} .
$$

Finally, if $G$ is a group that acts on the space $\mathcal{S}$ and bundles $\mathcal{N} S, \mathcal{N}^{\mu}$, and $F$, and preserves $h, \tilde{h}_{t}, \Omega$, and $K$, then $\varphi_{t}$ is $G$-equivariant.

Proof. With $\epsilon$ as provided by Lemma 3.21, let $\delta>0$ be as provided by Lemma 3.22. Then,

$$
\begin{gathered}
F_{t}:\left\{(b, \vec{n}, \sigma, v):\left.(b, \vec{n}, v) \in \Omega(\delta)\right|_{K},|\sigma|<\epsilon\right\} \longrightarrow \Omega(\delta) \times \mathbb{R}^{k}, \\
F_{t}(b, \vec{n}, \sigma, v)=\left(b, \vec{n}, v, \tilde{h}_{t}(b, \vec{n}, v)\right),
\end{gathered}
$$

is a diffeomorphism onto an open subset $W$ of the target space. The inverse of $F_{t}$ must have the form

$$
F_{t}^{-1}(b, \vec{n}, v, \tilde{\sigma})=\left(b, \vec{n}, \phi_{t}(b, \vec{n}, v, \tilde{\sigma}), v\right)
$$

for some smooth function $\phi_{t}$. By Lemma 3.22, $\left.\Omega(\delta)\right|_{K} \times\{0\} \subset W$. Thus,

$$
\varphi_{t} \in \Gamma\left(\left.\Omega(\delta)\right|_{K} ; \pi^{*} \mathcal{N}^{\mu}\right), \quad \varphi_{t}(b, \vec{n}, v)=\phi_{t}(b, \vec{n}, v, 0),
$$

is a well-defined section, and by definition of $\phi_{t}$,

$$
\left.\Omega(\delta)\right|_{K} \longrightarrow \tilde{h}_{t}^{-1}(0), \quad(b, \vec{n}, v) \longrightarrow\left(b, \vec{n}, \varphi_{t}(b, \vec{n}, v), v\right),
$$

is a diffeomorphism. It is orientation-preserving by Lemma 3.21. The estimate on $\varphi_{t}$ follows from the three properties of $h$, the first property of $\tilde{h}_{t}$, and the proof of Lemma 3.20. The final statement of the lemma is clear, since our construction commutes with the $G$-action. 
3.8. The Orientation of $\mathcal{M}_{\Sigma, t \nu, \lambda}(\mu)$ and the Gluing Map. At this point, our treatments of regular and semiregular cases diverge. In this subsection, we assume that $\mathcal{T}=(\Sigma,[N], I ; j, \underline{\lambda})$ is a semiregular bubble type and $\mu$ is an $N$-tuple of constraints in general position as defined below. Let $\lambda=\sum \lambda_{i}$ as before. We recall how each element of $\mathcal{M}_{\Sigma, t \nu, \lambda}(\mu)$ is assigned a sign and then specialize to the elements $\tilde{b}_{t \nu}(v) \in \mathcal{M}_{\Sigma, t \nu, \lambda}(\mu)$. We conclude this subsection with Theorem 3.29 that describes the elements of $\mathcal{M}_{\Sigma, t \nu, \lambda}(\mu)$ lying near the space $\mathcal{M}_{\mathcal{T}}(\mu)$.

\section{Definition 3.24.}

(1) Section

$$
\nu \in \Gamma^{0,1}\left(\Sigma \times V ; \Lambda_{J, j}^{0,1} \pi_{\Sigma}^{*} T^{*} \Sigma \otimes \pi_{V}^{*} T V\right)
$$

is $\lambda$-regular if for all $t \in(0,1)$ and $u \in \mathcal{M}_{\Sigma, t \nu, \lambda}$, the operator

$$
\left(D_{V, u}-\nabla^{V} \nu\right): \Gamma(u) \longrightarrow \Gamma^{0,1}(u)
$$

is surjective.

(2) If $\nu$ is $\lambda$-regular, $N$-tuple $\mu$ of oriented submanifolds of $V$ is $\nu$-regular if for all $t \in(0,1)$,

$$
\begin{aligned}
\bigoplus_{l \in[N]} T_{u_{\hat{0}}\left(y_{l}\right)} V & =\left.\operatorname{Im} d \mathrm{ev}_{[N]}\right|_{b}+\bigoplus_{l \in[N]} T_{u_{\hat{0}}\left(y_{l}\right)} \mu_{l} \\
\forall b & =\left(\Sigma,[N],\{\hat{0}\} ;,(\hat{0}, y), u_{\hat{0}}\right) \in \mathcal{M}_{\Sigma, t \nu, \lambda}(\mu),
\end{aligned}
$$

where

$$
\begin{gathered}
\left.\operatorname{dev}_{[N]}\right|_{b}: \operatorname{ker}\left(D_{V, u_{\hat{0}}}-\nabla^{V} . \nu\right) \oplus \bigoplus_{l \in[N]} T_{y_{l}} \Sigma \longrightarrow \bigoplus_{l \in[N]} T_{u_{\hat{0}}\left(y_{l}\right)} V \\
\left.\operatorname{dev}_{l}\right|_{b}\left(\xi, w_{[N]}\right)=\xi\left(y_{l}\right)+\left.d u_{\hat{0}}\right|_{y_{l}} w_{l} .
\end{gathered}
$$

(3) If $\mathcal{T}$ is a $(V, J)$-semiregular bubble type, tuple $\mu$ of oriented submanifolds of $V$ is $\mathcal{T}$-regular if

$$
\begin{aligned}
\bigoplus_{l \in[N]} T_{u_{j_{l}}\left(y_{l}\right)} V=\left.\operatorname{Im} d \operatorname{dev}_{[N]}\right|_{b}+\bigoplus_{l \in[N]} T_{u_{j_{l}}\left(y_{l}\right)} \mu_{l} \\
\forall b=(\Sigma,[N], I ; x,(j, y), u) \in \mathcal{H}_{\mathcal{T}}(\mu),
\end{aligned}
$$

where

$$
\begin{aligned}
\left.\operatorname{dev}_{[N]}\right|_{b}: \mathcal{K}_{b} \mathcal{T} & \longrightarrow \bigoplus_{l \in[N]} T_{u_{j_{l}}\left(y_{l}\right)} V, \\
\left.\operatorname{dev}_{l}\right|_{b}\left(\xi_{I}, w_{\hat{I} \sqcup[N]}\right) & =\xi_{j_{l}}\left(y_{l}\right)+\left.d u_{j_{l}}\right|_{y_{l}} w_{l} .
\end{aligned}
$$

(4) If $\mathcal{T}$ is a $(V, J)$-semiregular bubble type, $\mathcal{S} \subset \mathcal{M}_{\mathcal{T}}$ is a smooth submanifold, and $\tilde{\mathcal{S}} \subset \mathcal{M}_{\mathcal{T}}^{(0)}$ is the preimage of $\mathcal{S}$ under the quotient projection 
map, $N$-tuple $\mu$ of oriented submanifolds of $V$ is $\mathcal{S}$-regular if

$$
\begin{aligned}
& \bigoplus_{l \in[N]} T_{u_{j_{l}}\left(y_{l}\right)} V=\left.\operatorname{dev}[N]\right|_{b}\left(\mathcal{K}_{b} \mathcal{T} \cap T_{b} \tilde{\mathcal{S}}\right)+\bigoplus_{l \in[N]} T_{u_{j_{l}}\left(y_{l}\right)} \mu_{l} \\
& \forall b \in \tilde{\mathcal{S}}(\mu) \equiv \tilde{\mathcal{S}} \cap \mathcal{M}_{\mathcal{T}}^{(0)}(\mu) .
\end{aligned}
$$

Note that all four definitions above are independent of the choice of metrics on $V$. Throughout this subsection, we assume that $\nu$ is $\lambda$-regular, $\mathcal{T}$ is semiregular, and $\mu$ is $\nu$ - and $\mathcal{T}$-regular.

The space $\mathcal{M}_{\Sigma, t \nu, \lambda}$ consists of the maps $u: \Sigma \longrightarrow V$ such that

$$
\left.\bar{\partial} u\right|_{z}=t \nu(z, u(z)) \quad z \in \Sigma .
$$

Thus, the tangent space at $u$ can be described as

$$
T_{u} \mathcal{M}_{\Sigma, t \nu, \lambda}=\left\{\xi \in \Gamma\left(\Sigma ; u^{*} T V\right): D_{V, u} \xi-t L_{\nu, u} \xi=0\right\},
$$

where $L_{\nu, u} \xi$ is defined by

$$
\left\{L_{\nu, u} \xi\right\}(z)=\left.\nabla_{\xi(z)}^{V} \nu\right|_{(z, u(z))} .
$$

The operator $D_{V, u}-t L_{\nu, u}$ is independent of the choice of the connection along $\mathcal{M}_{\Sigma, t \nu, \lambda}$ and by assumption has no cokernel if $t \in(0,1)$. An orientation on $\mathcal{M}_{\Sigma, t \nu, \lambda}$ is determined by an orientation of the bundle $\Lambda_{\mathbb{R}}^{t o p} T \mathcal{M}_{\Sigma, t \nu, \lambda}$ over $\mathcal{M}_{\Sigma, t \nu, \lambda}$, which is the determinant line bundle of the elliptic operator $D_{V, u}-t L_{\nu, u}$. Since $L_{\nu, u}$ has order zero, the operator $D_{V, u}-t L_{\nu, u}$ is homotopic through elliptic operators to the operator $D_{V, u}$. Thus, $\Lambda_{\mathbb{R}}^{t o p} T \mathcal{M}_{\Sigma, t \nu, \lambda}$ is homotopic to

$$
\operatorname{det}\left(D_{V, u}\right)=\left(\Lambda_{\mathbb{R}}^{\text {top }}\left(\operatorname{ker} D_{V, u}\right)\right) \otimes\left(\Lambda_{\mathbb{R}}^{\text {top }}\left(\operatorname{coker} D_{V, u}\right)\right) ;
$$

see $[\mathbf{L M}]$. Since $D_{V, u}$ commutes with $J$, ker $D_{V, u}$ and coker $D_{V, u}$ are both complex vector spaces and thus have natural orientations, which induce an orientation on the determinant line bundle of $D_{V, u}$ and via a homotopy of operators on the determinant bundle of $D_{V, u}-t L_{\nu, u}$. It follows that $\mathcal{M}_{\Sigma, t \nu, \lambda} \times \Sigma^{N}$ is naturally oriented. If $\mu$ is a $\nu$-regular tuple of submanifold of $V$ of total codimension

$$
\begin{aligned}
\operatorname{codim} \mu & =\operatorname{dim} \mathcal{M}_{\Sigma, t \nu, \lambda} \times \Sigma^{N}=\text { ind } D_{V, u}+2|N| \\
& =2\left(\left\langle c_{1}(V, J), \lambda\right\rangle+\left(\operatorname{dim}_{\mathbb{C}} V\right)(1-g(\Sigma))+|N|\right),
\end{aligned}
$$

the differential of the map

$$
\mathrm{ev}_{[N]}: \mathcal{M}_{\Sigma, t \nu, \lambda} \times \Sigma^{N} \longrightarrow \prod_{l \in[N]} V, \quad\left(\Sigma,[N],\{\hat{0}\} ;,(\hat{0}, y), u_{\hat{0}}\right) \longrightarrow\left(u_{\hat{0}}\left(y_{l}\right)\right)_{l \in[N]},
$$

i.e., $\operatorname{dev}_{[N]}$ as defined in (2) of Definition 3.24, induces an isomorphism between $T \mathcal{M}_{\Sigma, t \nu, \lambda} \oplus T \Sigma^{N}$ and the normal bundle of $\mu$ in $V^{N}$ at each point 
of $\mathcal{M}_{\Sigma, t \nu, \lambda}(\mu)$. Here we identify the $N$-tuple $\mu$ with the submanifold

$$
\prod_{l \in[N]} \mu_{l} \subset \prod_{l \in[N]} V \equiv V^{N}
$$

Since the normal bundle of $\mu$ is oriented, the evaluation map also induces an orientation on $T \mathcal{M}_{\Sigma, t \nu, \lambda} \oplus T \Sigma^{N}$ along $\mathcal{M}_{\Sigma, t \nu, \lambda}(\mu)$. Each element $b \in$ $\mathcal{M}_{\Sigma, t \nu, \lambda}(\mu)$ is assigned a plus sign or is positively oriented if the two orientations agree, and a minus sign otherwise.

For any $v \in F^{(\emptyset)} \mathcal{T}$ such that $q_{v}$ is defined, let

$$
L_{\nu, v}: \Gamma\left(u_{v}\right) \longrightarrow \Gamma^{0,1}\left(u_{v}\right)
$$

be given by

$$
\left\{L_{\nu, v} \xi\right\}(z)=\left.\nabla_{\xi(z)}^{b_{v}} \nu\right|_{(z, f(z))}
$$

Denote by $\Gamma_{t,+}^{0,1}(v)$ the image of $\Gamma_{+}(v)$ under the map $D_{v}-t L_{\nu, v}$.

Lemma 3.25. For any compact subset $K$ of $\mathcal{M}_{\mathcal{T}}^{(0)}$, there exist $\delta, C>0$ such that for all $\left.v \in F^{(\emptyset)} \mathcal{T}_{\delta}\right|_{K}$ and $t \in(0, \delta)$,

(1) $\|\xi\|_{v, p, 1} \leq C\left\|D_{v} \xi-t L_{\nu, v} \xi\right\|_{v, p}$ for all $\xi \in \Gamma_{+}(v)$;

(2) $L^{p}(v)=\Gamma_{t,+}^{0,1}(v) \oplus \Gamma_{-}^{0,1}(v)$;

(3) if $D_{v, t}^{--}$and $L_{\nu, v, t}^{--}$are the $(-,-)$-components of $D_{v}$ and $L_{\nu, v}$ with respect to the decompositions $L_{1}^{p}(v)=\Gamma_{+}(v) \oplus \Gamma_{-}(v)$ and $L^{p}(v)=$ $\Gamma_{t,+}^{0,1}(v) \oplus \Gamma_{-}^{0,1}(v)$, then

$$
\begin{aligned}
\pi_{v,-}: \operatorname{ker}\left\{D_{v}-t L_{\nu, v}:\right. & \left.L_{1}^{p}(v) \longrightarrow L^{p}(v)\right\} \\
& \longrightarrow \operatorname{ker}\left\{D_{v, t}^{--}-t L_{\nu, v, t}^{--}: \Gamma_{-}(v) \longrightarrow \Gamma_{-}^{0,1}(v)\right\}
\end{aligned}
$$

is an orientation-preserving isomorphism, provided one of the two operators is surjective.

Proof. (1) The first claim is immediate from (1) of Lemma 3.16 and (2) of Lemma 3.5. The second is obtained by the same argument as in the proof of (3) of Lemma 3.16.

(2) By construction, $\pi_{v,-}$ is an isomorphism of the two kernels of the lemma. In particular, $D_{v}-t L_{\nu, v}$ is surjective if and only if $D_{v, t}^{--}-t L_{\nu, v, t}^{--}$is. Define

$$
\Phi_{\tau}: L_{1}^{p}(v) \oplus \Gamma_{-}^{0,1}(v) \longrightarrow L^{p}(v) \quad \text { and } \quad \Psi_{\tau}: \Gamma_{-}(v) \oplus \Gamma_{-}^{0,1}(v) \longrightarrow \Gamma_{-}^{0,1}(v)
$$

by

$$
\Phi_{\tau}(\xi, \eta)=D_{v} \xi+\tau t L_{\nu, v} \xi+\eta \quad \text { and } \quad \Psi_{\tau}(\xi, \eta)=\tau\left(D_{v, t}^{--}+t L_{\nu, v, t}^{--}\right) \xi+\eta .
$$

The first map is surjective for all $\tau \in[0,1]$ by (2) of the lemma, while the surjectivity of the second map is immediate from the definition. Furthermore, 
the maps

$$
\begin{gathered}
\phi_{\tau}: \operatorname{ker} \Phi_{\tau} \longrightarrow \Gamma_{-}(v), \phi_{\tau}(\xi, \eta)=\pi_{v,-} \xi, \quad \text { and } \\
\psi_{\tau}: \operatorname{ker} \Psi_{\tau} \longrightarrow \Gamma_{-}(v), \psi_{\tau}(\xi, \eta)=\xi,
\end{gathered}
$$

are isomorphisms such that

$$
\begin{gathered}
\psi_{1}^{-1} \phi_{1}(\xi, 0)=\pi_{v,-} \xi, \quad \text { if } \phi_{1}(\xi, \eta)=\psi_{1}\left(\xi^{\prime}, \eta^{\prime}\right), \eta=\eta^{\prime} ; \\
\text { and } \quad \psi_{0}^{-1} \phi_{0} J=J \psi_{0}^{-1} \phi_{0} .
\end{gathered}
$$

It follows that $\pi_{v,-}$ is an orientation-preserving map between the two kernels of the lemma.

If $K$ is a precompact open subset of $\mathcal{M}_{\mathcal{T}}$ and $\delta>0$ is such that $\tilde{b}_{t \nu}(v)$ is defined for all $\left.v \in F^{\emptyset} \mathcal{T}_{\delta}\right|_{K}$ and $t \in(0, \delta)$, let $\mathcal{M}(K, \delta)$ and $\tilde{\mathcal{M}}_{t \nu}(K, \delta)$ denote the images of $\left.F^{\emptyset} \mathcal{T}_{\delta}\right|_{K}$ under the maps $\gamma_{\mathcal{T}}$ and $\tilde{\gamma}_{\mathcal{T}, t \nu}$, respectively. Both maps are continuous and injective; see Subsection 4.2. The smooth structure of $F \mathcal{T}$ induces smooth structures on $\mathcal{M}(K, \delta)$ and $\tilde{\mathcal{M}}_{t \nu}(K, \delta)$, with the tangent bundles described by

$$
\begin{gathered}
T_{b(v)} \mathcal{M}(K, \delta)=\left\{\zeta_{\varpi}^{\prime}=\left.\frac{d}{d \tau} \zeta_{\tau \varpi}\right|_{\tau=0}: \varpi \in T_{v} F \mathcal{T}\right\} \oplus \bigoplus_{l \in[N]} T_{y_{l}(v)} \Sigma ; \\
T_{\tilde{b}_{t \nu}(v)} \tilde{\mathcal{M}}_{t \nu}(K, \delta)=\left\{\tilde{\zeta}_{\varpi}^{\prime}=\left.\frac{d}{d \tau} \tilde{\zeta}_{\tau \varpi}\right|_{\tau=0}: \varpi \in T_{v} F \mathcal{T}\right\} \oplus \bigoplus_{l \in[N]} T_{y_{l}(v)} \Sigma,
\end{gathered}
$$

where $T_{v} F \mathcal{T}$ denotes $T_{v} F^{\emptyset} \mathcal{T}$; see Subsection 3.4. It is easy to see that $\varpi \longrightarrow \zeta_{\varpi}^{\prime}$ is nearly complex linear and $\pi_{v,-}$ is almost the identity on the first component of $T_{b(v)} \mathcal{M}(K, \delta)$; both error terms are bounded by $C_{K}|v|$. Furthermore, by (1) of Lemma 3.6 and Corollary $4.7, \varpi \longrightarrow \tilde{\zeta}_{\varpi}^{\prime}$ also nearly computes with the complex structures and $\Pi_{b_{v}, \xi_{v}, t \nu} \pi_{v,-} \Pi_{b_{v}, \xi_{v, t \nu}}^{-1}$ is almost the identity on the first component of $T_{\tilde{b}_{t \nu}(v)} \tilde{\mathcal{M}}_{t \nu}(K, \delta)$; in the given case, the error terms are bounded by $C_{K}\left(t+|v|^{\frac{1}{p}}\right)$. Thus, the orientations of $\mathcal{M}(K, \delta)$ and $\tilde{\mathcal{M}}_{t \nu}(K, \delta)$ induced by the natural orientation of $F \mathcal{T}$ agree with the orientations induced from the natural orientation on $\Gamma_{-}(v) \oplus \bigoplus_{l \in[N]} T_{y_{l}(v)} \Sigma$ via the maps $\pi_{v,-} \oplus i d$ and $\pi_{v,-} \Pi_{b_{v}, \xi_{v}, t \nu}^{-1} \oplus i d$, respectively.

By the construction in Subsection 3.6,

$$
\tilde{\psi}_{t \nu}: \tilde{\mathcal{M}}_{t \nu}(K, \delta) \longrightarrow \Gamma^{0,1},\left.\quad v \longrightarrow t \nu\right|_{\tilde{u}_{v, t \nu}}-\bar{\partial} \tilde{u}_{v, t \nu} \in \Gamma^{0,1}\left(\tilde{u}_{v, t \nu}\right),
$$

determines a section of the bundle $\Pi \Gamma_{-}^{0,1}$ over $\tilde{\mathcal{M}}_{t \nu}(K, \delta)$, given by

$$
\Pi \Gamma_{-}^{0,1}\left(\tilde{b}_{t \nu}(v)\right)=\Pi_{b_{v}, \xi_{v}, t \nu} \Gamma_{-}^{0,1}(v) .
$$

Note that the zero set of this section is precisely the space

$$
\left(\mathcal{M}_{\Sigma, t \nu, \lambda} \times \Sigma^{N}\right) \cap \tilde{\mathcal{M}}_{t \nu}(K, \delta) .
$$


A linearization of this section is given by

$$
\begin{aligned}
\nabla_{\tilde{\zeta}_{\varpi}^{\prime}}\left(t \nu-\bar{\partial} \tilde{u}_{v, t \nu}\right) & \equiv \Pi_{b_{v}, \xi_{v, t \nu}} \pi_{v, t,-}^{0,1} \nabla_{\pi_{v,-} \Pi_{b_{v}, \xi_{v}, t \nu}^{-1} \tilde{\zeta}_{\varpi}^{\prime}}^{\prime} \Pi_{b_{v}, \xi_{v}, t \nu}^{-1}\left(t \nu-\bar{\partial} \tilde{u}_{v, t \nu}\right) \\
& =-\Pi_{b_{v}, \xi_{v, t \nu}}\left(D_{v, t}^{--}-t L_{v, \nu, t}^{--}\right) \pi_{v,-} \Pi_{b_{v}, \xi_{v, t \nu}}^{-1} \tilde{\zeta}_{\varpi}^{\prime}
\end{aligned}
$$

where

$$
\pi_{v, t,-}^{0,1}: L^{p}(v)=\Gamma_{+, t}^{0,1}(v) \oplus \Gamma_{-}^{0,1}(v) \longrightarrow \Gamma_{-}^{0,1}(v)
$$

is the projection map.

Corollary 3.26. For any compact subset $K$ of $\mathcal{M}_{\mathcal{T}}^{(0)}$, there exists $\delta>0$ such that for all $t \in(0, \delta)$, the orientation of $\left(\mathcal{M}_{\Sigma, t \nu, \lambda} \times \Sigma^{N}\right) \cap \tilde{\mathcal{M}}_{t \nu}(K, \delta)$ as the zero set of the section $\tilde{\psi}_{t \nu}$ agrees with its natural orientation.

Proof. Suppose $\tilde{b}_{t \nu}(v) \in\left(\mathcal{M}_{\Sigma, t \nu, \lambda} \times \Sigma^{N}\right) \cap \tilde{\mathcal{M}}_{t \nu}(K, \delta)$. Since we can use any connection in $\tilde{u}_{v, t \nu}^{*} T V$ to define the natural orientation on $T_{\tilde{u}_{v, t \nu}} \mathcal{M}_{\Sigma, t \nu, \lambda}$, we can write

$$
\left\{D_{\tilde{u}_{v, t \nu}}-t L_{\nu, \tilde{u}_{v, t \nu}}\right\} \xi=\Pi_{b_{v}, \xi_{v}, t \nu}\left\{D_{v}-t L_{\nu, v}\right\} \Pi_{b_{v}, \xi_{v, t \nu}}^{-1} \xi \quad \forall \xi \in \Gamma\left(\tilde{u}_{v, t}\right) .
$$

Thus, by Lemma $3.25, \pi_{v,-} \circ \Pi_{b_{v}, \xi_{v, t \nu}}^{-1} \oplus i d$ induces an orientation-preserving isomorphism

$$
T_{\tilde{b}_{t \nu}(v)} \mathcal{M}_{\Sigma, t \nu, \lambda} \oplus \bigoplus_{l \in[N]} T_{y_{l}(v)} \Sigma \longrightarrow \operatorname{ker}\left(D_{v, t}^{--}-L_{\nu, v, t}^{--}\right) \oplus \bigoplus_{l \in[N]} T_{y_{l}(v)} \Sigma,
$$

with the natural orientations on the two spaces. By the preceding paragraph, the same is true for the zero set of $\tilde{\psi}_{t \nu}$.

If $\mu$ is an $N$-tuple of constraints as above, let

$$
\begin{gathered}
\mathcal{M}(K, \delta ; \mu)=\left\{b(v) \in \mathcal{M}(K, \delta): \operatorname{ev}_{[N]}(b(v)) \in \mu\right\}, \\
\tilde{\mathcal{M}}_{t \nu}(K, \delta ; \mu)=\left\{\tilde{b}_{t \nu}(v) \in \tilde{\mathcal{M}}_{t \nu}(K, \delta): \operatorname{ev}_{[N]}\left(\tilde{b}_{t \nu}(v)\right) \in \mu\right\} .
\end{gathered}
$$

Then $\mathcal{M}(K, \delta ; \mu)$ and $\tilde{\mathcal{M}}_{t \nu}(K, \delta ; \mu)$ are smooth manifolds. In fact, the smoothness of $\mathcal{M}(K, \delta ; \mu)$ is immediate from the smoothness of $\left.F \mathcal{T}\right|_{\mathcal{M}_{\mathcal{T}}(\mu)}$, which is a consequence of $\mathcal{T}$-regularity of $\mu$. On the other hand, the smoothness of $\tilde{\mathcal{M}}_{t \nu}(K, \delta ; \mu)$ follows from Lemma 3.28 below. Furthermore, since $\mu$ is $\nu$-regular, the section $\tilde{\psi}_{t \nu}$ is transversal to zero in $\Pi \Gamma_{-}^{0,1}$ over $\tilde{\mathcal{M}}_{t \nu}(K, \delta ; \mu)$. By Corollary 3.26, the sign of $\tilde{b}_{t \nu}(v) \in \mathcal{M}_{\Sigma, t \nu, \lambda}(\mu)$ defined at the beginning of this subsection is its sign as an element of the zero set of the section $\tilde{\psi}_{t \nu}$ of $\Pi \Gamma_{-}^{0,1}$ over $\tilde{\mathcal{M}}_{t \nu}(K, \delta ; \mu)$.

$$
\begin{aligned}
& \text { If } b=(\Sigma,[N], I ; x,(j, y), u) \in \mathcal{M}_{\mathcal{T}}^{(0)}(\mu) \text {, let } \\
& \qquad \mathcal{K}_{b}^{\mu} \mathcal{T}=\left\{\left(\xi, w_{\hat{I} \sqcup[N]}\right) \in \mathcal{K}_{b} \mathcal{T}: \xi_{j_{l}}\left(y_{l}\right)+\left.d u_{j_{l}}\right|_{y_{l}} w_{l} \in T_{u_{j_{l}}\left(y_{l}\right)} \mu_{l} \forall l \in[N]\right\} .
\end{aligned}
$$


Denote by $\mathcal{N}_{b}^{\mu} \mathcal{T}$ the $\left(L^{2}, b\right)$-orthogonal complement of $\mathcal{K}_{b}^{\mu} \mathcal{T}$ in $\mathcal{K}_{b} \mathcal{T}$. Note that by (3) of Definition 3.24,

$$
\bigoplus_{l \in[N]} T_{u_{j_{l}}\left(y_{l}\right)} V=\left.\operatorname{dev}_{[N]}\right|_{b}\left(\mathcal{N}_{b}^{\mu} \mathcal{T}\right) \oplus \bigoplus_{l \in[N]} T_{u_{j_{l}}\left(y_{l}\right)} \mu_{l}
$$

We denote by $\tilde{\mathcal{N}}^{\mu} \mathcal{T}$ the bundle over $\mathcal{M}_{\mathcal{T}}^{(0)}(\mu)$ with fibers $\mathcal{N}_{b}^{\mu} \mathcal{T}$ and by $\mathcal{N}^{\mu} \mathcal{T} \longrightarrow \mathcal{M}_{\mathcal{T}}(\mu)$ its quotient by the natural $G_{\mathcal{T}}$-action.

Suppose $\mathcal{S} \subset \mathcal{M}_{\mathcal{T}}$ is a smooth oriented submanifold such that $\mu$ is $\mathcal{S}$ regular. Denote by $\tilde{\mathcal{S}} \subset \mathcal{M}_{\mathcal{T}}^{(0)}$ the preimage of $\mathcal{S}$ under the quotient projection map. Let

$$
\mathcal{N} S \longrightarrow \mathcal{S} \quad \text { and } \quad \mathcal{N} \tilde{\mathcal{S}} \longrightarrow \tilde{\mathcal{S}}
$$

be the normal bundles. Choose an $\left(\mathcal{A}(\mathcal{T}) \ltimes G_{\mathcal{T}}\right)$-equivariant orientationpreserving identification $\tilde{\phi}_{\mathcal{S}}: \mathcal{N} \tilde{\mathcal{S}}_{\delta} \longrightarrow \mathcal{M}_{\mathcal{T}}^{(0)}$ of neighborhoods of $\tilde{\mathcal{S}}$ in $\mathcal{N} \tilde{\mathcal{S}}$ and $\mathcal{M}_{\mathcal{T}}^{(0)}$. Let

$$
\tilde{\Phi}_{\mathcal{S}}: \pi_{\mathcal{N} \tilde{\mathcal{S}}}^{*} F^{(0)} \mathcal{T} \longrightarrow F^{(0)} \mathcal{T}
$$

be an $\left(\mathcal{A}(\mathcal{T}) \ltimes G_{\mathcal{T}}\right)$-equivariant vector-bundle isomorphism covering $\tilde{\phi}_{\mathcal{S}}$ such that $\tilde{\Phi}_{\mathcal{S}}$ is the identity on $\tilde{\mathcal{S}}$. Let

$$
\phi_{\mathcal{S}}: \mathcal{N} S_{\delta} \longrightarrow \mathcal{M}_{\mathcal{T}} \quad \text { and } \quad \Phi_{\mathcal{S}}: \pi_{\mathcal{N} S}^{*} F \mathcal{T} \longrightarrow F \mathcal{T}
$$

be the maps induced by $\tilde{\phi}_{\mathcal{S}}$ and $\tilde{\Phi}_{\mathcal{S}}$, respectively. Put

$$
\mathcal{S}(\mu)=\mathcal{S} \cap \mathcal{M}_{\mathcal{T}}(\mu), \quad \tilde{\mathcal{S}}(\mu)=\tilde{\mathcal{S}} \cap \mathcal{M}_{\mathcal{T}}^{(0)}(\mu) .
$$

Since $\mu$ is $\mathcal{S}$-regular, we can choose an $\left(\mathcal{A}(\mathcal{T}) \ltimes G_{\mathcal{T}}\right)$-equivariant orientationpreserving identification $\tilde{\phi}_{\mathcal{S}}^{\mu}:\left.\tilde{\mathcal{N}}^{\mu} \mathcal{T}_{\delta}\right|_{\tilde{\mathcal{S}}(\mu)} \longrightarrow \tilde{\mathcal{S}}$. Let

$$
\tilde{\Phi}_{\mathcal{S}}^{\mu}: \pi_{\tilde{\mathcal{N}}^{\mu} \mathcal{T}}^{*}\left(\mathcal{N} \tilde{\mathcal{S}} \oplus F^{(0)} \mathcal{T}\right) \longrightarrow \mathcal{N} \tilde{\mathcal{S}} \oplus F^{(0)} \mathcal{T}
$$

be a $\left(\mathcal{A}(\mathcal{T}) \ltimes G_{\mathcal{T}}\right)$-equivariant splitting-preserving vector-bundle isomorphism covering $\tilde{\phi}_{\mathcal{S}}^{\mu}$ such that $\tilde{\Phi}_{\mathcal{S}}^{\mu}$ is the identity on $\tilde{\mathcal{S}}(\mu)$. Denote by

$$
\phi_{\mathcal{S}}^{\mu}:\left.\mathcal{N}^{\mu} \mathcal{T}_{\delta}\right|_{\mathcal{S}(\mu)} \longrightarrow \tilde{\mathcal{S}} \text { and } \Phi_{\mathcal{S}}^{\mu}: \pi_{\mathcal{N}^{\mu} \mathcal{T}}(\mathcal{N} S \oplus F \mathcal{T}) \longrightarrow \mathcal{N} S \oplus F \mathcal{T}
$$

the maps induced by $\tilde{\phi}_{\mathcal{S}}^{\mu}$ and $\tilde{\Phi}_{\mathcal{S}}^{\mu}$, respectively.

Definition 3.27. With notation as above and in Subsection $3.4,\left(\Phi_{\mathcal{S}}, \Phi_{\mathcal{S}}^{\mu}\right)$ is a regularization of $\mathcal{S}(\mu)$ if for all $b \in \tilde{\mathcal{S}}(\mu), \vec{n} \in \mathcal{N}_{b} \tilde{\mathcal{S}}_{\delta(b)}$, and $\sigma \in \tilde{\mathcal{N}}_{b}^{\mu} \mathcal{T}_{\delta(b)}$, there exists $\varpi(\vec{n}, \sigma) \in \mathcal{K}_{\tilde{\phi}_{\mathcal{S}}(b, \vec{n})} \mathcal{T}$ such that

$$
\tilde{\Phi}_{\mathcal{S}} \tilde{\Phi}_{\mathcal{S}}^{\mu}(b, \sigma ; \vec{n}, v)=\left\{\tilde{\Phi}_{\mathcal{S}}(b, \vec{n} ; v)\right\}(\varpi(\vec{n}, \sigma)) \quad \forall v \in F_{b}^{(0)} \mathcal{T} .
$$

Note that if $\mu$ is $\mathcal{S}$-regular, $\mathcal{S}(\mu)$ admits a normalization. In fact, we can start with any choice of $\Phi_{\mathcal{S}}$ and $\left.\Phi_{\mathcal{S}}^{\mu}\right|_{\pi_{\mathcal{N}^{\mu} \mathcal{T}^{\mathcal{N} S}}}$ as in the preceding paragraph, and then choose $\left.\Phi_{\mathcal{S}}^{\mu}\right|_{\pi_{\mathcal{N}^{\mu} \mathcal{T}}^{*} F \mathcal{T}}$ so that the triple satisfies the requirements of 
the definition. In applications of Theorem 3.29 in $[\mathbf{Z 2}]$, the exact choice of $\Phi_{\mathcal{S}}^{\mu}$ does not matter, but that of $\Phi_{\mathcal{S}}$ does play a role.

For the purposes of Theorem 3.29, we assume that $\Phi_{\mathcal{S}}$ and $\Phi_{\mathcal{S}}^{\mu}$ also encode the lifts of $\phi_{\mathcal{S}}$ and $\phi_{\mathcal{S}}^{\mu}$ to the bundles $\pi_{\mathcal{N} S}^{*} \Gamma_{-}^{0,1} \longrightarrow \mathcal{S}$ and $\pi_{\mathcal{N}^{\mu} \mathcal{T}}^{*} \Gamma_{-}^{0,1} \longrightarrow \mathcal{S}(\mu)$, respectively. Put

$$
\begin{aligned}
F^{(0)} \mathcal{S} & =\mathcal{N} \tilde{\mathcal{S}} \oplus F^{(0)} \mathcal{T}, \quad F^{(\emptyset)} \mathcal{S}=\left\{(b, \vec{n}, v) \in F^{(0)} \mathcal{S}: v \in F_{b}^{(\emptyset)} \mathcal{T}\right\} ; \\
F \mathcal{S} & =\mathcal{N} S \oplus F \mathcal{T}, \quad F^{\emptyset} \mathcal{S}=\left\{[b, \vec{n}, v] \in F^{(0)} \mathcal{S}:[b, v] \in F_{b}^{\emptyset} \mathcal{T}\right\} .
\end{aligned}
$$

Lemma 3.28. For any $\left(\mathcal{A}(\mathcal{T}) \ltimes G_{\mathcal{T}}\right)$-invariant precompact open subset $K$ of $\tilde{\mathcal{S}}(\mu)$, there exist an open neighborhood $U_{K}$ of $K$ in $\mathcal{M}_{\mathcal{T}}^{(0)}$ and $\delta, C>0$ with the following property. If $t \in(0, \delta)$, there exists a smooth $\left(\mathcal{A}(\mathcal{T}) \ltimes G_{\mathcal{T}}\right)$ equivariant section

$$
\tilde{\varphi}_{\mathcal{S}, t \nu}^{\mu} \in \Gamma\left(\left.F^{(\emptyset)} \mathcal{S}_{\delta}\right|_{K} ; \pi_{F^{(0)}}^{*} \tilde{\mathcal{S}}^{\mu} \mathcal{T}\right)
$$

such that $\left\|\tilde{\varphi}_{\mathcal{S}, t \nu}^{\mu}(v)\right\|_{b_{v}, C^{0}} \leq C\left(t+|v|^{\frac{1}{p}}\right)$ for all $\left.v \in F^{(\emptyset)} \mathcal{S}_{\delta}\right|_{K}$ and

$$
\left.F^{\emptyset} \mathcal{S}_{\delta}\right|_{K} \longrightarrow \tilde{\mathcal{M}}_{t \nu}\left(U_{K}, \delta ; \mu\right), \quad[b, \vec{n}, v] \longrightarrow \tilde{\gamma}_{\mathcal{T}, t \nu}\left(\tilde{\Phi}_{\mathcal{S}}\left(\tilde{\Phi}_{\mathcal{S}}^{\mu} \tilde{\varphi}_{\mathcal{S}, t \nu}^{\mu}(b, \vec{n}, v)\right)\right),
$$

is an orientation-preserving diffeomorphism.

Proof. Since $\mu$ is a regular value of $\left.\mathrm{ev}_{[N]}\right|_{\mathcal{S}}$ and $K$ is precompact, there exists $\delta>0$ such that the map

$$
\begin{gathered}
\left\{\left.(b, \vec{n}, v, \sigma) \in F^{(0)} \mathcal{S} \oplus \tilde{\mathcal{N}}^{\mu} \mathcal{T}\right|_{K}:|\vec{n}|,\|\sigma\|_{b, C^{0}}<\delta\right\} \longrightarrow F^{(0)} \mathcal{T} \\
(b, \vec{n}, v, \sigma) \longrightarrow \tilde{\Phi}_{\mathcal{S}} \tilde{\Phi}_{\mathcal{S}}^{\mu}(b, \sigma ; \vec{n}, v),
\end{gathered}
$$

is an $\left(\mathcal{A}(\mathcal{T}) \ltimes G_{\mathcal{T}}\right)$-equivariant orientation-preserving diffeomorphism onto its image. Thus, if $\delta>0$ is sufficiently small, there exists $C>0$ such that, with notation as in Definition 3.27,

$$
\begin{aligned}
C^{-1}\left\|\sigma-\sigma^{\prime}\right\|_{b, C^{0}} \leq\left\|\varpi(\vec{n}, \sigma)-\varpi\left(\vec{n}, \sigma^{\prime}\right)\right\|_{b, C^{0}} \leq C\left\|\sigma-\sigma^{\prime}\right\|_{b, C^{0}} \\
\forall b \in \tilde{\mathcal{S}}(\mu), \vec{n} \in \mathcal{N}_{b} \tilde{\mathcal{S}}_{\delta}, \sigma, \sigma^{\prime} \in \tilde{\mathcal{N}}_{b}^{\mu} \mathcal{T} .
\end{aligned}
$$

Then by Corollary 4.11 and definition of $S_{\varpi}^{\prime}$ in Subsection 3.4,

$$
\begin{aligned}
& \mid d_{V}\left(\tilde{\phi}_{\mathcal{S}} \tilde{\Phi}_{\mathcal{S}}^{\mu}(b, \sigma ; \vec{n}), \tilde{\gamma}_{\mathcal{T}, t \nu}\left(\tilde{\Phi}_{\mathcal{S}} \tilde{\Phi}_{\mathcal{S}}^{\mu}(b, \sigma ; \vec{n}, v)\right)\right) \\
& -d_{V}\left(\tilde{\phi}_{\mathcal{S}} \tilde{\Phi}_{\mathcal{S}}^{\mu}\left(b, \sigma^{\prime} ; \vec{n}\right), \tilde{\gamma}_{\mathcal{T}, t \nu}\left(\tilde{\Phi}_{\mathcal{S}} \tilde{\Phi}_{\mathcal{S}}^{\mu}\left(b, \sigma^{\prime} ; \vec{n}, v\right)\right)\right) \mid \leq C\left(t+|v|^{\frac{1}{p}}\right)\left\|\sigma-\sigma^{\prime}\right\|_{b, C^{0}} \\
& \forall t \in(0, \delta), b \in \tilde{\mathcal{S}}(\mu), \vec{n} \in \mathcal{N}_{b} \tilde{\mathcal{S}}_{\delta}, \sigma, \sigma^{\prime} \in \tilde{\mathcal{N}}_{b}^{\mu} \mathcal{T}, v \in F_{b}^{(\emptyset)} \mathcal{T}_{\delta}
\end{aligned}
$$

On a neighborhood of $\operatorname{ev}_{[N]}(b) \in \mu$, we can identify the normal bundle of $\mu$ in $V^{N} g_{V}$-isometrically with the trivial hermitian bundle of the same rank. 
Let $\pi$ denote the projection onto the fiber. Since $\mu$ is $\mathcal{S}$-regular,

$$
\begin{aligned}
&\left\|\sigma-\sigma^{\prime}\right\|_{b, C^{0}} \leq C\left|\pi \operatorname{ev}_{[N]}\left(\tilde{\phi}_{\mathcal{S}} \tilde{\Phi}_{\mathcal{S}}^{\mu}(b, \sigma ; \vec{n})\right)-\pi \operatorname{ev}_{[N]}\left(\tilde{\phi}_{\mathcal{S}} \tilde{\Phi}_{\mathcal{S}}^{\mu}\left(b, \sigma^{\prime} ; \vec{n}\right)\right)\right| \\
& \forall b \in \tilde{\mathcal{S}}(\mu), \vec{n} \in \mathcal{N}_{b} \tilde{\mathcal{S}}_{\delta}, \sigma, \sigma^{\prime} \in \tilde{\mathcal{N}}_{b}^{\mu} \mathcal{T} .
\end{aligned}
$$

Thus, we can apply Corollary 3.23 to

$$
h=\pi \circ \operatorname{ev}_{[N]} \circ \tilde{\phi}_{\mathcal{S}} \circ \tilde{\Phi}_{\mathcal{S}}^{\mu} \quad \text { and } \quad \tilde{h}_{t}=\pi \circ \operatorname{ev}_{[N]} \circ \tilde{\gamma}_{\mathcal{T}, t \nu} \circ \tilde{\Phi}_{\mathcal{S}} \circ \tilde{\Phi}_{\mathcal{S}}^{\mu} .
$$

We obtain $\delta, \epsilon>0$ and for each $t \in(0, \delta)$ a section $\tilde{\varphi}_{\mathcal{S}, t \nu}^{\mu}$ with the claimed bound such that the map

$$
\begin{gathered}
\left.F^{(\emptyset)} \mathcal{S}_{\delta}\right|_{K} \longrightarrow\left\{(b, \vec{n}, v, \sigma):\|\sigma\|_{b, C^{0}}<\epsilon, \operatorname{ev}_{[N]} \tilde{\gamma}_{\mathcal{T}, t \nu}\left(\tilde{\Phi}_{\mathcal{S}}\left(\tilde{\Phi}_{\mathcal{S}}^{\mu}(b, \vec{n} ; \sigma, v)\right)\right) \in \mu\right\}, \\
(b, \vec{n}, v) \longrightarrow\left(b, \vec{n}, v, \tilde{\varphi}_{\mathcal{S}, t \nu}^{\mu}(b, \vec{n}, v)\right),
\end{gathered}
$$

is an orientation-preserving diffeomorphism. Since

$$
\begin{gathered}
\left\{[b, \vec{n}, v, \sigma]:[b, \vec{n}, v] \in F^{\emptyset} \mathcal{S}_{\delta},\|\sigma\|_{b, C^{0}}<\epsilon\right\} \longrightarrow \tilde{\mathcal{M}}_{t \nu}\left(U_{K}, \delta\right), \\
{[b, \vec{n}, \sigma, v] \longrightarrow \tilde{\gamma}_{\mathcal{T}, t \nu}\left(\tilde{\Phi}_{\mathcal{S}}\left(\tilde{\Phi}_{\mathcal{S}}^{\mu}(b, \vec{n} ; \sigma, v)\right)\right),}
\end{gathered}
$$

is orientation-preserving by the discussion above and our assumptions on $\tilde{\phi}_{\mathcal{S}}$, the claim follows. Above

$$
U_{K}=\tilde{\phi}_{\mathcal{S}}\left(\tilde{\Phi}_{\mathcal{S}}^{\mu}\left(\left\{\left.(b, \vec{n}, \sigma) \in \mathcal{N} \tilde{\mathcal{S}} \oplus \tilde{\mathcal{N}}^{\mu} \mathcal{T}\right|_{K}:\|\vec{n}\|_{b, C^{0}}<\epsilon,\|\sigma\|_{b, C^{0}}<\epsilon\right\}\right)\right) .
$$

Theorem 3.29. Suppose $\lambda \in H_{2}(V ; \mathbb{Z}), \mathcal{T}=(\Sigma,[N], I ; j, \underline{\lambda})$ is a $(V, J)$ semiregular bubble type, with $\sum_{i \in I} \lambda_{i}=\lambda$ and cokernel bundle $\Gamma_{-}^{0,1} \longrightarrow \mathcal{M}_{\mathcal{T}}$, and $\left(\tilde{\Gamma}_{-}, \Gamma_{-}^{0,1}, R\right)$ is an obstruction bundle setup. Let $\mathcal{S} \subset \mathcal{M}_{\mathcal{T}}$ be a smooth oriented submanifold,

$$
\nu \in \Gamma^{0,1}\left(\Sigma \times V ; \Lambda_{J, j}^{0,1} \pi_{\Sigma}^{*} T^{*} \Sigma \otimes \pi_{V}^{*} T V\right)
$$

a $\lambda$-regular section, $\mu$ a $\nu$-, $\mathcal{T}$-, and $\mathcal{S}$-regular $N$-tuple of submanifolds of $V$ of total codimension

$$
\operatorname{codim} \mu=2\left(\left\langle c_{1}(V, J), \lambda\right\rangle+\left(\operatorname{dim}_{\mathbb{C}} V\right)(1-g(S))+|N|\right),
$$

and $\left(\Phi_{\mathcal{S}}, \Phi_{\mathcal{S}}^{\mu}\right)$ is regularization of $\mathcal{S}(\mu)$. Then for every precompact open subset $K$ of $\mathcal{S}(\mu)$, there exist a neighborhood $U_{K}$ of $K$ in $\bar{C}_{(\lambda ; N)}^{\infty}(\Sigma ; \mu)$ and $\delta, \epsilon, C>0$ with the following property. For every $t \in(0, \epsilon)$, there exist a section

$$
\varphi_{\mathcal{S}, t \nu}^{\mu} \in \Gamma\left(\left.F^{\emptyset} \mathcal{S}_{\delta}\right|_{K} ; \pi_{F \mathcal{S}}^{*} \mathcal{N}^{\mu} \mathcal{T}\right), \quad \text { with } \quad\left\|\varphi_{\mathcal{S}, t \nu}^{\mu}(v)\right\|_{b_{v}, C^{0}} \leq C\left(t+|v|^{\frac{1}{p}}\right)
$$


and a sign-preserving bijection between $\mathcal{M}_{\Sigma, t \nu, \lambda}(\mu) \cap U_{K}$ and the zero set of the section $\psi_{\mathcal{S}, t \nu}^{\mu}$ defined by

$$
\begin{aligned}
& \psi_{\mathcal{S}, t \nu}^{\mu} \in \Gamma\left(\left.F^{\emptyset} \mathcal{S}_{\delta}\right|_{K} ; \pi_{F \mathcal{S}}^{*} \Gamma_{-}^{0,1}\right), \\
& \Phi_{\mathcal{S}}^{\mu}\left(\varphi_{\mathcal{S}, t \nu}^{\mu}(v) ; \psi_{\mathcal{S}, t \nu}^{\mu}(v)\right)=\psi_{\mathcal{S}, t \nu}\left(\Phi_{\mathcal{S}}^{\mu}\left(\varphi_{\mathcal{S}, t \nu}^{\mu}(v)\right)\right) ; \\
& \psi_{\mathcal{S}, t \nu} \in \Gamma\left(\left.F^{\emptyset} \mathcal{S}_{\delta}\right|_{\mathcal{S} \cap U_{K}} ; \pi_{F \mathcal{S}}^{*} \Gamma_{-}^{0,1}\right), \\
& \Phi_{\mathcal{S}}\left(v ; \psi_{\mathcal{S}, t \nu}(v)\right)=\psi_{\mathcal{T}, t \nu}\left(\Phi_{\mathcal{S}}(v)\right) ; \\
& \psi_{\mathcal{T}, t \nu} \in \Gamma\left(\left.F^{\emptyset} \mathcal{T}_{\delta}\right|_{\mathcal{M}_{\mathcal{T} \cap U_{K}}} ; \pi_{F \mathcal{T}}^{*} \Gamma_{-}^{0,1}\right), \\
& R_{v} \psi_{\mathcal{T}, t \nu}(v)=\pi_{v,-}^{0,1}\left(t \nu_{v, t}-\bar{\partial}\left(u_{b_{v}} \circ q_{v}\right)-D_{v} \xi_{v, t \nu}-\tilde{\eta}_{v, t \nu}\right) \text {, }
\end{aligned}
$$

for some $\nu_{v, t}, \tilde{\eta}_{v, t \nu} \in \Gamma^{0,1}\left(u_{v}\right)$ and $\xi_{v, t \nu} \in \tilde{\Gamma}_{+}(v)$, dependent smoothly on $v$, such that

$$
\begin{gathered}
\left\|\nu_{v, t}-\nu\right\|_{v, 2} \leq C\left(t+|v|^{\frac{1}{p}}\right), \quad\left\|\xi_{v, t \nu}\right\|_{v, p, 1} \leq C\left(t+|v|^{\frac{1}{p}}\right), \\
\text { and } \quad\left\|\tilde{\eta}_{v, t \nu}\right\|_{v, p} \leq C\left(t+|v|^{\frac{1}{p}}\right)^{2} .
\end{gathered}
$$

Furthermore, if $z \in \Sigma$ and $\left(B_{b_{v}}\left(u_{v}(z), C \delta\right), J, g_{V, b_{v}}\right)$ is isometric to a ball in $\mathbb{C}^{n}$, then $\tilde{\eta}_{v, t \nu}(z)=0$.

Remark. In specific applications, the main goal would be to express the number of zeros of $\psi_{\mathcal{S}, t \nu}^{\mu}$ in terms of the cohomology ring of a closure of $\mathcal{S}_{\mathcal{T}}(\mu)$. One of the significant intermediate steps is to extract the leadingorder terms from the section $\psi_{\mathcal{S}, t \nu}^{\mu}$. If $\lambda_{\hat{0}}=0$, the estimate on $\nu_{v, t}$ given above easily leads to a sufficiently good estimate on $\pi_{v,-}^{0,1} \nu_{v, t}$; see $[\mathbf{I}]$ and $[\mathbf{Z 2}]$. In such a case, one can also extract the first-order term from $\pi_{v,-}^{0,1} \bar{\partial} u_{v}$, which suffices for the computation in $[\mathbf{I}]$. A power-series expansion for $\pi_{v,-}^{0,1} \bar{\partial} u_{v}$ is given in $[\mathbf{Z 2}]$, where terms of up to third degree are used. With the choice of metrics in $[\mathbf{Z 2}]$, the term $\pi_{v,-}^{0,1} \tilde{\eta}_{v, t \nu}$ vanishes. The remaining term is shown to be secondary for a good choice of the obstruction bundle setup.

Proof of Theorem 3.29. Let $\delta, \epsilon>0$ be as in Lemma 3.28 and its proof. We take $\varphi_{\mathcal{S}, t \nu}^{\mu}$ to be the section descendant from the $G_{\mathcal{T}}$-equivariant section $\tilde{\varphi}_{\mathcal{S}, t \nu}^{\mu}$. Denote by $U_{K}^{\prime}$ the open set $U_{K}$ of Lemma 3.28. By Corollary 4.22, there exists a neighborhood $U_{K}$ of $K$ in $\bar{C}_{(\lambda ; N)}^{\infty}(\Sigma ; \mu)$ such that

$$
\mathcal{M}_{\Sigma, t \nu, \lambda}(\mu) \cap U_{K}
$$

is contained in $\tilde{\mathcal{M}}_{t \nu}\left(U_{K}^{\prime}, \delta ; \mu\right)$. The neighborhood $U_{K}$ can always be chosen to contain all the zeros of the section $\tilde{\psi}_{t \nu}$ of the bundle $\Pi \Gamma_{-}^{0,1}$ over $\mathcal{M}_{\Sigma, t \nu, \lambda}(\mu) \cap U_{K}$. By Corollary 3.26, $\mathcal{M}_{\Sigma, t \nu, \lambda}(\mu) \cap U_{K}$ is precisely the oriented zero set of the section $\tilde{\psi}_{t \nu}$. Since the map

$$
\left.F^{\emptyset} \mathcal{S}_{\delta}\right|_{K} \longrightarrow \tilde{\mathcal{M}}_{t \nu}\left(U_{K}^{\prime}, \delta ; \mu\right), \quad v \longrightarrow \tilde{\gamma}_{\mathcal{T}, t \nu}\left(\Phi_{\mathcal{S}} \Phi_{\mathcal{S}}^{\mu}\left(\varphi_{\mathcal{S}, t \nu}^{\mu}(v)\right)\right),
$$


is an orientation-preserving diffeomorphism by Lemma 3.28, it induces a sign-preserving bijection between the zero set of $\tilde{\psi}_{t \nu}$ on $\tilde{\mathcal{M}}_{t \nu}\left(U_{K}^{\prime}, \delta ; \mu\right)$, and the zero set of the section

$$
\left(\tilde{\gamma}_{\mathcal{T}, t \nu} \Phi_{\mathcal{S}} \Phi_{\mathcal{S}}^{\mu} \varphi_{\mathcal{S}, t \nu}^{\mu}\right)^{*} \tilde{\psi}_{t \nu} \in \Gamma\left(\left.F^{\emptyset} \mathcal{S}_{\delta}\right|_{K} ;\left(\tilde{\gamma}_{\mathcal{T}, t \nu} \Phi_{\mathcal{S}} \Phi_{\mathcal{S}}^{\mu} \varphi_{\mathcal{S}, t \nu}^{\mu}\right)^{*} \Pi \Gamma_{-}^{0,1}\right)
$$

By equation (3.17), under the canonical identification

$$
\left(\tilde{\gamma}_{\mathcal{T}, t \nu} \gamma_{\mathcal{T}}^{-1}\right)^{*} \Pi \Gamma_{-}^{0,1}=\Gamma_{-}^{0,1} \longrightarrow \mathcal{M}\left(U_{K}^{\prime}, \delta\right),
$$

the section $\left(\tilde{\gamma}_{\mathcal{T}, t \nu} \gamma_{\mathcal{T}}^{-1}\right)^{*} \tilde{\psi}_{t \nu}$ corresponds to the section $\psi_{t \nu}$ given by

$$
\begin{aligned}
\psi_{t \nu}(b(v)) & =\left.t \nu\right|_{u_{v}}-\bar{\partial} u_{v}-\tilde{\pi}_{v,+}^{0,1} \eta_{v, t \nu}-N_{v, t \nu} P_{v} \eta_{v, t \nu} \\
& =\pi_{v,-}^{0,1}\left(\left.t \nu\right|_{u_{v}}-\bar{\partial} u_{v}-\tilde{\pi}_{v,+}^{0,1} \eta_{v, t \nu}-N_{v, t \nu} P_{v} \eta_{v, t \nu}\right) \\
& =\pi_{v,-}^{0,1}\left(t \nu_{v, \xi_{v, t \nu}}-\bar{\partial} u_{v}-\tilde{\pi}_{v,+}^{0,1} \eta_{v, t \nu}-N_{v} P_{v} \eta_{v, t \nu}\right) .
\end{aligned}
$$

The second equality above is automatic, since $\psi_{t \nu}(b(v)) \in \Gamma_{-}^{0,1}(v)$; the third follows from the definition of $N_{v, t \nu}$ in Subsection 3.6. The bounds on the terms $\nu_{v, \xi_{v}, t \nu}, \eta_{v, t \nu}, N_{v} P_{v} \eta_{v, t \nu}$ also follow from Subsection 3.6. By definition of $\Gamma_{-}^{0,1}$ in Subsection 3.4 and equation (3.19), under the canonical identification

$$
\gamma_{\mathcal{T}}^{*} \Gamma_{-}^{0,1}=\left.\pi_{F \mathcal{T}}^{*} \Gamma_{-}^{0,1} \longrightarrow F^{\emptyset} \mathcal{T}_{\delta}\right|_{U_{K}^{\prime}},
$$

the section $\gamma_{\mathcal{T}}^{*} \psi_{t \nu}$ corresponds to the section $\psi_{\mathcal{T}, t \nu}$, described in the statement of the theorem.

The next proposition describes a special case of the above theorem. It is obtained by fixing a metric $g$ on $\Sigma$ and going through the construction analogous to that in Subsection 3.6 and then modification for constraints as above. The sign statement below follows from the fact that the $\left(L^{2}, g, g_{V}\right)$ projection $\operatorname{ker}\left(D_{V, u_{b}}-\tau t L_{\nu, u_{b}}\right) \longrightarrow \operatorname{ker} D_{V, u_{b}}$ is an isomorphism for all $\tau \in$ $[0,1], t$ sufficiently small, and $b \in \mathcal{M}_{\Sigma, t \nu, \lambda^{*}}(\mu)$ sufficiently close to $K$.

Proposition 3.30. Suppose $\lambda \in H_{2}(V ; \mathbb{Z}), \mathcal{T}=(\Sigma,[N],\{\hat{0}\} ; \hat{0}, \lambda)$ is a $(V, J)$ regular bubble type,

$$
\nu \in \Gamma^{0,1}\left(\Sigma \times V ; \Lambda_{J, j}^{0,1} \pi_{\Sigma}^{*} T^{*} \Sigma \otimes \pi_{V}^{*} T V\right)
$$

is any section, and $\mu$ is a $\mathcal{T}$-regular $N$-tuple of submanifolds of $V$ of total codimension

$$
\operatorname{codim} \mu=2\left(\left\langle c_{1}(V, J), \lambda\right\rangle+\left(\operatorname{dim}_{\mathbb{C}} V\right)(1-g(S))+|N|\right) .
$$

Then $\mathcal{M}_{\mathcal{T}}(\mu)$ is a discrete set and for every finite subset $K$ of $\mathcal{S}(\mu)$, there exist a neighborhood $U_{K}$ of $K$ in $\bar{C}_{(\lambda ; N)}^{\infty}(\Sigma ; \mu), \epsilon>0$, and for each $t \in(0, \epsilon)$ a sign-preserving bijection between $K$ and $\mathcal{M}_{\Sigma, t \nu, \lambda}(\mu) \cap U_{K}$. 
3.9. Gluing Maps for Spaces $\overline{\mathcal{U}}_{\mathcal{T}}^{(0)}(\mu)$ and Orientations. We now consider the case $\mathcal{T}=\left(S^{2}, M, I ; j, \underline{\lambda}\right)$ is a regular bubble type. However, most of the analysis in this subsection applies to any regular bubble type $\mathcal{T}$. Let $\mu$ be a generic $\tilde{M}$-tuple of submanifolds in $V$, as defined below. If $I=\bigsqcup_{k \in K} I_{k}$ is the decomposition of $I$ into rooted trees and $\left\{\mathcal{T}_{k}\right\}$ are the corresponding simple types derived from $\mathcal{T}$, the product gluing map,

$$
\left(\tilde{\gamma}_{\mathcal{T}_{k}}\right)_{k \in K}: \prod_{k \in K} F \mathcal{T}_{k, \delta_{k}} \longrightarrow \prod_{k \in K} \overline{\mathcal{M}}_{\left\langle\mathcal{T}_{k}\right\rangle},
$$

may not map the total space of the bundle over $\mathcal{U}_{\mathcal{T}}^{(0)}(\mu)$ into $\overline{\mathcal{U}}_{\langle\mathcal{T}\rangle}^{(0)}(\mu)$. In this subsection, we remedy this deficiency of the product gluing map. We also show that all the spaces $\overline{\mathcal{U}}_{\langle\mathcal{T}\rangle}^{(0)}(\mu)$ and $\overline{\mathcal{U}}_{\langle\mathcal{T}\rangle}(\mu)$ are naturally oriented topological orbifolds and the gluing maps defined below preserve orientations.

Definition 3.31. If $\mathcal{T}$ is a $(V, J)$-regular bubble type, $\tilde{M}$-tuple $\mu$ of oriented submanifolds of $V$ is $\mathcal{T}$-regular if the manifolds $\left\{\mu_{l}: l \in \tilde{M}-M\right\}$ intersect transversally in $V$ and

$$
\begin{aligned}
T_{\mathrm{ev}(b)} V \oplus \bigoplus_{l \in \tilde{M} \cap M} T_{u_{j_{l}}\left(y_{l}\right)} V=\left.\operatorname{Im} d \mathrm{ev}\right|_{b}+\left.\operatorname{Im} d \operatorname{ev}_{\tilde{M} \cap M}\right|_{b} & +T_{\operatorname{ev}(b)} \bigcap_{l \in \tilde{M}-M} \mu_{l} \\
& +\bigoplus_{l \in \tilde{M} \cap M} T_{u_{j_{l}}\left(y_{l}\right)} \mu_{l}
\end{aligned}
$$

for all $b=\left(S^{2}, M, I ; x,(j, y), u\right) \in \mathcal{B}_{\mathcal{T}}(\mu)$.

Let $\mathcal{T}, \mathcal{T}_{k}, K, \mu$, and $b$ be as above. Denote by

$$
b_{k}=\left(S^{2}, M_{k}, I_{k} ;\left.x\right|_{\hat{I}_{k}},\left.(j, y)\right|_{M_{k}},\left.u\right|_{I_{k}}\right)
$$

the corresponding $\mathcal{T}_{k}$-bubble map; see Subsection 2.5. Let $\mathcal{N}_{b}^{\mu} \mathcal{T}$ be the $\left(L^{2}, b\right)$-orthogonal complement of

$$
\begin{array}{r}
\tilde{\mathcal{K}}_{b}^{\mu} \mathcal{T}=\left\{\left(\xi, w_{\hat{I} \sqcup M}\right) \in \tilde{\mathcal{K}}_{b} \mathcal{T}: \xi_{j_{l}}\left(y_{l}\right)+\left.d u_{j_{l}}\right|_{y_{l}} w_{l} \in T_{u_{j_{l}}\left(y_{l}\right)} \mu_{l} \forall l \in \tilde{M} \cap M,\right. \\
\left.\xi_{i}(\infty) \in T_{\operatorname{ev}(b)} \bigcap_{l \in \tilde{M}-M} \mu_{l} \forall i \in I-\hat{I}\right\}
\end{array}
$$

in $\bigoplus_{k \in K} \mathcal{K}_{b_{k}} \mathcal{T}_{k}$. Denote by $\tilde{\mathcal{N}}^{\mu} \mathcal{T} \longrightarrow \mathcal{B}_{\mathcal{T}}(\mu)$ and $\mathcal{N}^{\mu} \mathcal{T} \longrightarrow \mathcal{U}_{\mathcal{T}}^{(0)}(\mu)$ the corresponding vector bundles. Let

$$
\begin{gathered}
\mathcal{N}^{\mu} \mathcal{B}_{\mathcal{T}}=\tilde{\mathcal{N}}^{\mu} \mathcal{T} \oplus(\mathbb{C} \oplus \mathbb{R})^{K} \longrightarrow \mathcal{B}_{\mathcal{T}}(\mu), \\
\mathcal{N}^{\mu} \mathcal{U}_{\mathcal{T}}^{(0)}=\mathcal{N}^{\mu} \mathcal{T} \oplus(\mathbb{C} \oplus \mathbb{R})^{K} \longrightarrow \mathcal{U}_{\mathcal{T}}^{(0)}(\mu) ; \\
F^{(0)} \mathcal{T}=\bigoplus_{k \in K} F^{(0)} \mathcal{T}_{k} \longrightarrow \mathcal{B}_{\mathcal{T}}, \quad F \mathcal{T}=\bigoplus_{k \in K} F \mathcal{T}_{k} \longrightarrow \mathcal{U}_{\mathcal{T}}^{(0)}
\end{gathered}
$$


The last two vector bundles carry norms induced from the norms on $F^{(0)} \mathcal{T}_{k}$, while we define norms on the first two by

$$
|(b, \sigma,(c, r))|=\|\sigma\|_{b, C^{0}}+|(c, r)|
$$

if $\sigma \in \mathcal{N}_{b}^{\mu} \mathcal{T} \subset \bigoplus_{k \in K} \tilde{\mathcal{K}}_{b_{k}} \mathcal{T}_{k}$ and $(c, r) \in(\mathbb{C} \oplus \mathbb{R})^{K}$. If $\delta$ is sufficiently small, define

$$
\begin{gathered}
\tilde{\phi}_{\mathcal{T}}^{\mu}: \mathcal{N}^{\mu} \mathcal{B}_{\mathcal{T}, \delta} \longrightarrow \prod_{k \in K} \mathcal{M}_{\mathcal{T}_{k}}^{(0)} \quad \text { by } \\
\tilde{\phi}_{\mathcal{T}}^{\mu}(\sigma,(c, r))=\left(\left(c_{k}, r_{k}\right) \cdot H_{\mathcal{T}_{k}}^{(0)}\left(\sigma_{k}\right)\right)_{k \in K} \in \prod_{k \in K} \mathcal{M}_{\mathcal{T}_{k}}^{(0)}
\end{gathered}
$$

where $H_{\mathcal{T}_{k}}^{(0)}$ is as at the end of Subsection 3.2 and $\left(c_{k}, r_{k}\right)$. denotes the action of a neighborhood of

$$
0 \in \mathbb{C} \times \mathbb{R}=\mathbb{C} \times \mathbb{R} \times\{0\} \subset \mathbb{C} \times \mathbb{R} \times \mathbb{R}
$$

described in Subsection 3.1. Since $\tilde{\phi}_{\mathcal{T}}^{\mu}$ is $\left(\mathcal{A}(\mathcal{T}) \ltimes \tilde{G}_{\mathcal{T}}\right)$-equivariant, it descends to a $G_{\mathcal{T}}^{*}$-equivariant map

$$
\phi_{\mathcal{T}}^{\mu}: \mathcal{N}^{\mu} \mathcal{U}_{\mathcal{T}, \delta}^{(0)} \longrightarrow \prod_{k \in K} \mathcal{M}_{\mathcal{T}_{k}}
$$

Let $\Phi_{\mathcal{T}}^{\mu}: \pi_{\mathcal{N}^{\mu} \mathcal{U}_{\mathcal{T}}}^{*} F \mathcal{T} \longrightarrow F \mathcal{T}$ be a $G_{\mathcal{T}^{-}}^{*}$ equivariant vector-bundle map covering the map $\phi_{\mathcal{T}}^{\mu}$ such that $\Phi_{\mathcal{T}}^{\mu}$ is the identity over $\mathcal{U}_{\mathcal{T}}^{(0)}(\mu)$. Denote by $\tilde{\Phi}_{\mathcal{T}}^{\mu}$ the lift of $\Phi_{\mathcal{T}}^{\mu}$ to $\mathcal{N}^{\mu} \mathcal{B}_{\mathcal{T}, \delta}$. Let $\Phi_{\mathcal{T}, k}^{\mu}$ and $\tilde{\Phi}_{\mathcal{T}, k}^{\mu}$ be $k$ th components of $\Phi_{\mathcal{T}}^{\mu}$ and $\tilde{\Phi}_{\mathcal{T}}^{\mu}$, respectively.

Lemma 3.32. With assumptions and notation as above, there exist $(\mathcal{A}(\mathcal{T}) \ltimes$ $\left.\tilde{G}_{\mathcal{T}}\right)$-invariant functions $\delta, C \in C^{\infty}\left(\mathcal{B}_{\mathcal{T}}(\mu) ; \mathbb{R}^{+}\right)$and an $\left(\mathcal{A}(\mathcal{T}) \ltimes \tilde{G}_{\mathcal{T}}\right)$ - equivariant section

$$
\tilde{\varphi}_{\mathcal{T}}^{\mu} \in \Gamma\left(F^{(0)} \mathcal{T}_{\delta} ; \pi_{F(0)}^{*} \mathcal{T}^{\mu} \mathcal{B}_{\mathcal{T}, \delta}\right),
$$

such that $\left|\tilde{\varphi}_{\mathcal{T}}^{\mu}(v)\right| \leq C\left(b_{v}\right)|v|^{\frac{1}{p}}$ and

$$
F \mathcal{T}_{\delta} \longrightarrow \overline{\mathcal{U}}_{\langle\mathcal{T}\rangle}^{(0)}(\mu), \quad v \longrightarrow\left(\tilde{\gamma}_{\mathcal{T}_{k}}\left(\Phi_{\mathcal{T}, k}^{\mu} \varphi_{\mathcal{T}}^{\mu}(v)\right)\right)_{k \in K},
$$

is a homeomorphism onto an open neighborhood of $\mathcal{U}_{\mathcal{T}}^{(0)}(\mu)$ in $\overline{\mathcal{U}}_{\langle\mathcal{T}\rangle}^{(0)}(\mu)$. Furthermore, the restriction of this map to $F^{\emptyset} \mathcal{T}_{\delta}$ is an orientation-preserving diffeomorphism onto an open subset of $\mathcal{U}_{\langle\mathcal{T}\rangle}^{(0)}(\mu)$.

Proof. Denote by $\mathcal{N}_{\mathcal{T}} \mu$ the normal bundle of

$$
\begin{aligned}
X_{\mathcal{T}}(\mu) & \equiv\left\{x_{K} \in V^{K}: x_{k_{1}}=x_{k_{2}} \in \mu_{l} \forall k_{1}, k_{2} \in K, l \in \tilde{M}-M\right\} \times \prod_{l \in \tilde{M} \cap M} \mu_{l} \\
& \subset V^{K} \times V^{\tilde{M} \cap M} .
\end{aligned}
$$


Let $\tilde{\mathcal{N}}_{\mathcal{T}} \mu=\mathcal{N} X_{\mathcal{T}} \mu \oplus(\mathbb{C} \oplus \mathbb{R})^{K}$. Since the $\tilde{G}_{\mathcal{T}}$-action does not change any evaluation maps and the constraints are in general position, the differential of the map

$$
\begin{gathered}
\Psi_{\mathcal{T}, \tilde{M}}: \prod_{k \in K} \mathcal{M}_{\mathcal{T}_{k}}^{(0)} \longrightarrow V^{K} \times V^{\tilde{M} \cap M} \times(\mathbb{C} \times \mathbb{R})^{K}, \\
\Psi_{\mathcal{T}, \tilde{M}}=\left(\left(\operatorname{ev}_{\mathcal{T}_{k}}\left(b_{k}\right)\right)_{k \in K} ;\left(\operatorname{ev}_{l}(b)\right)_{l \in M \cap \tilde{M}} ;\left(\Psi_{\mathcal{T}_{k}, \hat{0}_{I_{k}}}\left(b_{k}\right)\right)_{k \in K}\right),
\end{gathered}
$$

where $\Psi_{\mathcal{T}_{k}, \hat{0}_{I_{k}}}\left(b_{k}\right) \in \mathbb{C} \times \mathbb{R}$ is as in Subsection 3.2, induces an isomorphism between $\mathcal{N}_{b}^{\mu} \mathcal{B}_{\mathcal{T}}$ and $\tilde{\mathcal{N}}_{\mathcal{T}} \mu$. This isomorphism is orientation-preserving by definition of orientations. Thus,

$$
\tilde{\phi}_{\mathcal{T}}^{\mu}: \mathcal{N}^{\mu} \mathcal{B}_{\mathcal{T}, \delta} \longrightarrow \prod_{k \in K} \mathcal{M}_{\mathcal{T}_{k}}^{(0)}
$$

is an orientation-preserving diffeomorphism onto an open neighborhood of $\mathcal{B}_{\mathcal{T}}(\mu)$ in $\prod_{k \in K} \mathcal{M}_{\mathcal{T}_{k}}^{(0)}$, provided $\delta \in C^{\infty}\left(\mathcal{B}_{\mathcal{T}}(\mu) ; \mathbb{R}^{+}\right)$is sufficiently small. By the same argument as in Subsection 3.8, for any simple bubble type $\mathcal{T}^{\prime}$, the map

$$
\tilde{\gamma}_{\mathcal{T}^{\prime}}: F^{\emptyset} \mathcal{T}_{\delta}^{\prime} \longrightarrow \mathcal{M}_{\left\langle\mathcal{T}^{\prime}\right\rangle}=\mathcal{H}_{\left\langle\mathcal{T}^{\prime}\right\rangle}
$$

is an orientation-preserving diffeomorphism onto an open subset of $\mathcal{M}_{\left\langle\mathcal{T}^{\prime}\right\rangle}$ provided $\delta \in C^{\infty}\left(\mathcal{M}_{\mathcal{T}} ; \mathbb{R}^{+}\right)$is sufficiently small. Along with Corollary 4.23, this implies that the product map

$$
\prod_{k \in K} \tilde{\gamma}_{\mathcal{T}_{k}}: \prod_{k \in K} F \mathcal{T}_{k, \delta} \longrightarrow \prod_{k \in K} \overline{\mathcal{M}}_{\left\langle\mathcal{T}_{k}\right\rangle}
$$

is a homeomorphism onto an open neighborhood of $\prod_{k \in K} \mathcal{M}_{\mathcal{T}_{k}}$ in $\prod_{k \in K} \overline{\mathcal{M}}_{\left\langle\mathcal{T}_{k}\right\rangle}$ and its restriction to the preimage of $\prod_{k \in K} \mathcal{M}_{\left\langle\mathcal{I}_{k}\right\rangle}$ is an orientation-preserving diffeomorphism. The lemma now follows by applying an argument similar to the proof of Lemma 3.28 to the functions

$$
h(v)=\Psi_{\mathcal{T}, \tilde{M}}\left(\tilde{\phi}_{\mathcal{T}}^{\mu}(\sigma,(c, r))\right), \quad \tilde{h}(v)=\tilde{h}_{0}(v)=\Psi_{\mathcal{T}, \tilde{M}}\left(\left(\tilde{\gamma}_{k} \Phi_{\mathcal{T}, k}^{\mu}(v)\right)_{k \in K}\right),
$$

where we write $v=(\sigma,(c, r), v)$, with $(\sigma,(c, r)) \in \mathcal{N}^{\mu} \mathcal{B}_{\mathcal{T}}$ and $v \in F^{(0)} \mathcal{T}$. Since $\mathcal{B}_{\mathcal{T}}(\mu)$ is generally not precompact in $\prod_{k \in K} \mathcal{M}_{\mathcal{T}_{k}}^{(0)}$, we end up with

$$
\delta, C \in C^{\infty}\left(\mathcal{B}_{\mathcal{T}}(\mu) ; \mathbb{R}^{+}\right),
$$

instead of $\delta, C \in \mathbb{R}^{+}$. Another difference is that $\tilde{h}$ is not necessarily smooth with respect to the standard smooth structure on $\mathcal{N}^{\mu} \mathcal{B}_{\mathcal{T}} \oplus F^{(0)} \mathcal{T}$. However, 
we can put a smooth structure on the total space such that the composite maps

$$
\begin{gathered}
\mathcal{N}^{\mu} \mathcal{B}_{\mathcal{T}, \delta} \oplus F^{(0)} \mathcal{T} \longrightarrow F^{(0)} \mathcal{T} \longrightarrow \mathbb{R}, \quad v \longrightarrow \tilde{\Phi}_{\mathcal{T}}^{\mu}(v) \\
v_{h} \longrightarrow\left|v_{h}\right|^{\frac{1}{3 p}}, \quad h \in \hat{I}_{k}, \quad k \in K,
\end{gathered}
$$

are smooth, whenever $\delta \in C^{\infty}\left(\mathcal{B}_{\mathcal{T}}(\mu) ; \mathbb{R}^{+}\right)$is sufficiently small. Then by Corollary 4.5, $\tilde{h}$ is $C^{2}$, which is sufficient for the arguments of Subsection 3.7. Finally, in the given case $\tilde{h}$ is defined on all of $\left(\mathcal{N}^{\mu} \mathcal{B}_{\mathcal{T}} \oplus F^{(0)} \mathcal{T}\right)_{\delta}$ and thus the second condition on $\tilde{h}_{t}$ in Subsection 3.7 is redundant.

Suppose $\mathcal{T}$ is a bubble type and $\mu$ is an $\tilde{M}$-tuple of constraints in general position. By Lemma 3.32, there exist $G_{\mathcal{T}}^{*}$-invariant functions $\delta, C \in$ $C^{\infty}\left(\mathcal{U}_{\mathcal{T}}^{(0)}(\mu) ; \mathbb{R}^{+}\right)$and a $G_{\mathcal{T}}^{*}$-equivariant section

$$
\varphi_{\mathcal{T}}^{\mu} \in\left(F \mathcal{T}_{\mu} ; \pi_{F \mathcal{T}}^{*} \mathcal{N}^{\mu} \mathcal{U}_{\mathcal{T}, \delta}^{(0)}\right)
$$

such that $\left|\varphi_{\mathcal{T}}^{\mu}(v)\right| \leq C\left(b_{v}\right)|v|^{\frac{1}{p}}$ and

$$
\tilde{\gamma}_{\mathcal{T}}^{\mu}: F \mathcal{T}_{\delta} \longrightarrow \overline{\mathcal{U}}_{\langle\mathcal{T}\rangle}^{(0)}(\mu), \quad \tilde{\gamma}_{\mathcal{T}}^{\mu}(v)=\left(\tilde{\gamma}_{\mathcal{T}_{k}}\left(\Phi_{\mathcal{T}, k}^{\mu} \varphi_{\mathcal{T}}^{\mu}(v)\right)\right)_{k \in K},
$$

is a homeomorphism onto a neighborhood of $\mathcal{U}_{\mathcal{T}}^{(0)}(\mu)$ in $\overline{\mathcal{U}}_{\langle\mathcal{T}\rangle}^{(0)}(\mu)$, which is an orientation-preserving diffeomorphism on a dense open subset of the domain. If $\mathcal{T}^{\prime}$ is another regular bubble type such that $\langle\mathcal{T}\rangle=\left\langle\mathcal{T}^{\prime}\right\rangle$ and $\mu$ is $\mathcal{T}^{\prime}$-regular, it follows that

$$
\tilde{\gamma}_{\mathcal{T}}^{\mu-1} \tilde{\gamma}_{\mathcal{T}^{\prime}}^{\mu}: \tilde{\gamma}_{\mathcal{T}^{\prime}}^{\mu-1}\left(\tilde{\gamma}_{\mathcal{T}}^{\mu}\left(F \mathcal{T}_{\delta}\right)\right) \longrightarrow \tilde{\gamma}_{\mathcal{T}}^{\mu-1}\left(\tilde{\gamma}_{\mathcal{T}^{\prime}}^{\mu}\left(F \mathcal{T}_{\delta^{\prime}}^{\prime}\right)\right)
$$

is a homeomorphism provided $\delta^{\prime} \in C^{\infty}\left(\mathcal{U}_{\mathcal{T}^{\prime}}^{(0)}(\mu) ; \mathbb{R}^{+}\right)$is sufficiently small. Furthermore, by the above it is orientation-preserving on a dense open subset of its domain. It follows that $\tilde{\gamma}_{\mathcal{T}}^{\mu-1} \tilde{\gamma}_{\mathcal{T}}^{\mu}$ is an orientation-preserving homeomorphism everywhere. We thus obtain

Theorem 3.33. Let $\mathcal{T}^{*}=\left(S^{2}, M, I^{*} ; j, \underline{\lambda}^{*}\right)$ be a basic bubble type and $\mu$ an $\tilde{M}$-tuple of constraints such that $\mu$ is $\mathcal{T}$-regular for every bubble type $\mathcal{T} \leq \mathcal{T}^{*}$.

(1) The spaces $\overline{\mathcal{U}}_{\mathcal{T}^{*}}^{(0)}(\mu)$ and $\overline{\mathcal{U}}_{\mathcal{T}^{*}}(\mu)$ are oriented topological orbifolds.

(2) Suppose $\mathcal{T}<\mathcal{T}^{*}, \phi_{\mathcal{T}}^{\mu}: \mathcal{N}^{\mu} \mathcal{T}_{\tilde{\delta}} \longrightarrow \mathcal{U}_{\mathcal{T}}^{(0)}$ is a $G_{\mathcal{T}^{*} \text {-equivariant identi- }}$ fication of neighborhoods of $\mathcal{U}_{\mathcal{T}}^{(0)}(\mu)$ in $\mathcal{N}^{\mu} \mathcal{T}$ and in $\mathcal{U}_{\mathcal{T}}^{(0)}$, and $\Phi_{\mathcal{T}}^{\mu}$ : $\pi_{\mathcal{N}^{\mu} \mathcal{T}}^{*} F \mathcal{T} \longrightarrow F \mathcal{T}$ is a lift of $\phi_{\mathcal{T}}^{\mu}$ such that $\left.\Phi_{\mathcal{T}}^{\mu}\right|_{\mathcal{U}_{\mathcal{T}}^{(0)}(\mu)}=1$. Then there

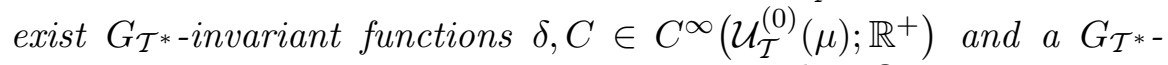
equivariant continuous orientation-preserving identification,

$$
\tilde{\gamma}_{\mathcal{T}}^{\mu}: F \mathcal{T}_{\delta} \longrightarrow \overline{\mathcal{U}}_{\mathcal{T}^{*}}^{(0)}(\mu)
$$


of neighborhoods of $\mathcal{U}_{\mathcal{T}}^{(0)}(\mu)$ in $F \mathcal{T}$ and in $\overline{\mathcal{U}}_{\mathcal{T}^{*}}^{(0)}(\mu)$, which is smooth on $F^{\emptyset} \mathcal{T}_{\delta} \longrightarrow \mathcal{U}_{\mathcal{T}^{*}}(\mu)$. Furthermore, for every $v \in F \mathcal{T}_{\delta}$, there exists $\sigma(v) \in \mathcal{N}^{\mu} \mathcal{T}$ such that

$$
\begin{gathered}
\|\sigma(v)\|_{b^{*}} \leq C\left(b^{*}\right)|v|^{\frac{1}{p}} \quad \text { and } \quad u_{\tilde{\gamma}_{\mathcal{T}}^{\mu}(v)}=\exp _{V, u_{b^{\prime}} \circ q_{\Phi_{\mathcal{T}}^{\mu}(\sigma(v))}} \xi_{v}, \\
\text { where } \quad \Phi_{\mathcal{T}}^{\mu}(\sigma(v)) \equiv\left[b^{\prime}, v^{\prime}\right] \\
\text { for some } \xi_{v} \in \Gamma\left(u_{b^{\prime}} \circ q_{\Phi_{\mathcal{T}}^{\mu}(\sigma(v))}\right) \text { with }\left\|\xi_{v}\right\|_{C^{0}} \leq C\left(b_{v}\right)|v|^{\frac{1}{p}} \text {. }
\end{gathered}
$$

Remark. The descriptive statement (2) of Theorem 3.33 is used in [Z2] for local excess-intersection type of computations on the spaces $\overline{\mathcal{U}}_{\mathcal{T} *}(\mu)$.

\section{Technical Issues}

4.1. Continuity of the Gluing Map. Let $\mathcal{T}=(S, M, I ; j, \underline{\lambda})$ be a simple regular bubble type and $H$ a nonempty subset of $\hat{I}$. Suppose $v_{k} \in F^{(\emptyset)} \mathcal{T}_{\delta}$ and the sequence $v_{k}$ converges to $v^{*} \in F^{(H)} \mathcal{T}_{\delta}$. In this subsection, we show that $\tilde{\gamma}_{\mathcal{T}}\left(v_{k}\right)$ converges to $\tilde{\gamma}_{\mathcal{T}}\left(v^{*}\right)$ in the Gromov topology. Our main interest is the case $S=S^{2}$.

It is sufficient to assume that $\pi_{h}\left(v_{k}\right)=\pi_{h}\left(v^{*}\right)$ if $h \notin H$. In particular, $b \equiv b_{v^{*}}=b_{v_{k}}$. Denote by $\tilde{\mathcal{T}}=\mathcal{T}(H)$ the bubble type of $b\left(v^{*}\right)$. For each $k$, define

$$
\tilde{v}_{k}=\left(\tilde{b}\left(v^{*}\right),\left(\tilde{v}_{k}\right)_{H}\right) \in F_{b\left(v^{*}\right)}^{(0)} \tilde{\mathcal{T}}
$$

as follows. If $h \in H$, put

$$
i_{H} h=\min \left\{i<h: \text { if } h^{\prime} \in I \& i<h^{\prime}<h, h^{\prime} \notin H\right\} .
$$

Since $I$ is a rooted tree, $i_{H} h$ is well-defined. Let

$$
\tilde{v}_{k, h}=\prod_{i_{H} h<h^{\prime} \leq h} v_{k, h^{\prime}}
$$

Since $v_{k} \longrightarrow v^{*}, \tilde{v}_{k, h} \longrightarrow 0$ for all $h \in H$. Furthermore, by construction $\Sigma_{v_{k}}=\Sigma_{\tilde{v}_{k}}$ and $q_{v_{k}}=q_{v^{*} \circ} q_{\tilde{v}_{k}}$. In particular, $u_{v_{k}}=u_{v^{*}} \circ q_{\tilde{v}_{k}}$.

For any $h \in H$ and $\delta>0$, let

$$
\begin{gathered}
A_{h, \delta, k}=q_{v_{k}}^{-1}\left(\left\{\left(\iota_{h}, z\right): r_{b, h}(z) \leq \delta\right\} \cup\left\{(h, z):\left|q_{S}^{-1}(z)\right| \leq \delta\right\}\right) \subset \Sigma_{v_{k}}, \\
A_{h, \delta}^{*}=q_{v^{*}}^{-1}\left(\left\{\left(\iota_{h}, z\right): r_{b, h}(z) \leq \delta\right\} \cup\left\{(h, z):\left|q_{S}^{-1}(z)\right| \leq \delta\right\}\right) \subset \Sigma_{v^{*}}, \\
\Sigma_{\delta}^{*}=\Sigma_{v^{*}}-\bigcup_{h \in H} A_{h, \delta}^{*} .
\end{gathered}
$$

It is convenient to make the following definitions. If $\eta_{k} \in L^{p}\left(v_{k}\right)$, the sequence $\left\{\eta_{k}\right\}$ converges to $\eta^{*} \in L^{p}\left(v^{*}\right)$ if $q_{\tilde{v}_{k}}^{-1 *} \eta_{k}$ converges to $\eta^{*}$ in the $L^{p}$-norm on all precompact open subsets of $\Sigma_{v^{*}}^{*}$ and

$$
\lim _{\delta \longrightarrow 0} \lim _{k \longrightarrow 0}\left\|\eta_{k}\right\|_{v_{k}, L^{p}\left(A_{h, \delta, k}\right)}=0 \quad \forall h \in H .
$$


If $\xi_{k} \in L_{1}^{p}\left(v_{k}\right)$, the sequence $\left\{\xi_{k}\right\}$ converges to $\xi^{*} \in L_{1}^{p}\left(v^{*}\right)$ if $\xi_{k} \circ q_{\tilde{v}_{k}}^{-1}$ converges to $\xi^{*}$ in the $L_{1}^{p}$-norm on all precompact open subsets of $\Sigma_{v^{*}}^{*}$ and

$$
\lim _{\delta \longrightarrow 0} \lim _{k \longrightarrow 0}\left\|\xi_{k}\right\|_{v_{k}, L_{1}^{p}\left(A_{h, \delta, k}\right)}=0 \quad \forall h \in H .
$$

In (4.1) and (4.2), we use the modified Sobolev norms.

Lemma 4.1. There exist $C, \delta \in C^{\infty}\left(\mathcal{M}_{\mathcal{T}}^{(0)} ; \mathbb{R}^{+}\right)$such that for any sequence $\left\{v_{k} \in F^{(\emptyset)} \mathcal{T}_{\delta}\right\}$ converging to $v^{*}$ as above and $\xi \in \Gamma_{+}\left(v^{*}\right)$

$$
\left\|\pi_{v_{k},-}\left(\xi \circ q_{\tilde{v}_{k}}\right)\right\|_{v_{k}, p, 1} \leq C(b)\left|v_{k}-v^{*}\right|\|\xi\|_{v^{*}, p, 1} .
$$

Proof. Note that $\Gamma_{-}\left(v_{k}\right)=\left\{\xi^{-} \circ q_{\tilde{v}_{k}}: \xi^{-} \in \Gamma\left(v^{*}\right)\right\}$. Thus, the difference $q_{\tilde{v}_{k}}^{*} \pi_{v^{*},-}-\pi_{v_{k},-} q_{\tilde{v}_{k}}^{*}$ arises entirely from the difference between the metrics $q_{\tilde{v}_{k}}^{*} g_{v^{*}}$ and $g_{v_{k}}$. By construction, the two metrics differ only on the annuli $A_{h, 2\left|v_{k, h}\right|^{\frac{1}{2}, k}}$ for $h \in H$. Thus, the claim follows from (2) of Lemma 3.5.

Lemma 4.2. If $\eta_{k}$ converges to $\eta^{*}$, then $P_{v_{k}} \eta_{k}$ converges to $P_{v^{*}} \eta^{*}$.

Proof. (1) Let $\left\{\epsilon_{k}\right\},\left\{\delta_{k}\right\} \subset \mathbb{R}^{+}$be sequences converging to zero such that

$$
\begin{gathered}
\left\|\eta^{*}\right\|_{v^{*}, L^{p}\left(A_{h, \delta_{k}}^{*}\right)} \leq \epsilon_{k} ; \quad\left\|P_{v^{*}} \eta^{*}\right\|_{v^{*}, L_{1}^{p}\left(A_{h, \delta_{k}}^{*}\right)} \leq \epsilon_{k}, \\
\lim _{k \longrightarrow \infty}\left\|\eta_{k}\right\|_{v_{k}, L^{p}\left(A_{h, \delta_{k^{*}, k}}\right)}<\epsilon_{k}
\end{gathered}
$$

for all $h \in H$. For every $k^{*}>0$, choose $N_{k^{*}}$ such that for all $k>N_{k^{*}}$

$$
\left\|q_{\tilde{v}_{k}}^{-1 *} \eta_{k}-\eta^{*}\right\|_{v^{*}, L^{p}\left(\Sigma_{k_{k^{*}}}^{*}\right)} \leq \epsilon_{k^{*}} \quad \text { and } \quad\left\|\eta_{k}\right\|_{v_{k}, L^{p}\left(A_{h, \delta_{k^{*}, k}}\right)} \leq \epsilon_{k^{*}}
$$

for all $h \in H$. It can be assumed that $2\left|v_{k}-v^{*}\right|^{\frac{1}{2}} \leq \delta_{k^{*}}, \epsilon_{k^{*}}$ whenever $k>N_{k^{*}}$. For any $k>N_{k^{*}}$, let $\tilde{\eta}_{k^{*}, k} \in L^{p}\left(v^{*}\right)$ be given by

$$
\tilde{\eta}_{k^{*}, k}= \begin{cases}q_{\tilde{v}_{k}}^{-1 *} \tilde{\eta}_{k}, & \text { on } \Sigma_{\delta_{k^{*}}^{*}}^{*} \\ 0, & \text { outside of } \Sigma_{\delta_{k^{*}}}^{*}\end{cases}
$$

Then $\left\|\tilde{\eta}_{k^{*}, k}\right\|_{v^{*}, p} \leq\left\|\eta_{k}\right\|_{v_{k}, p}$. Let

$$
\tilde{P}_{k^{*}, k} \eta_{k}=q_{\tilde{v}_{k}}^{*} P_{v^{*}} \tilde{\eta}_{k^{*}, k} \in L_{1}^{p}\left(v_{k}\right) .
$$

Then by Lemma 3.16 and the first assumptions of (4.3) and (4.4),

$$
\begin{aligned}
\left\|q_{\tilde{v}_{k}}^{-1 *} \tilde{P}_{k^{*}, k} \eta_{k}-P_{v^{*}} \eta^{*}\right\|_{v^{*}, L_{1}^{p}\left(\Sigma_{\delta_{k^{*}}}^{*}\right)} & \leq\left\|P_{v^{*}} \tilde{\eta}_{k^{*}, k}-P_{v^{*}} \eta^{*}\right\|_{v^{*}, p, 1} \\
& \leq C(b)\left\|\tilde{\eta}_{k^{*}, k}-\eta^{*}\right\|_{v^{*}, p} \leq 2 C(b) \epsilon_{k^{*}}
\end{aligned}
$$


Since $\left\|d q_{\tilde{v}_{k}}\right\|_{C^{0}} \leq C(b)$, by (4.3) and the first assumption of (4.4) for all $h \in H$,

$$
\begin{aligned}
& \left\|\tilde{P}_{k^{*}, k} \eta_{k}\right\|_{v_{k}, L_{1}^{p}\left(A_{\left.h, \delta_{k^{*}, k}\right)}\right.} \leq C(b)\left\|P_{v^{*}} \tilde{\eta}_{k^{*}, k}\right\|_{v^{*}, L_{1}^{p}\left(A_{h, \delta_{k^{*}}^{*}}^{*}\right)} \\
& \quad \leq C(b)\left(\left\|P_{v^{*}} \eta^{*}\right\|_{v^{*}, L_{1}^{p}\left(A_{h, \delta_{k^{*}}}^{*}\right)}+\left\|P_{v^{*}} \tilde{\eta}_{k^{*}, k}-P_{v^{*}} \eta^{*}\right\|_{v^{*}, p, 1}\right) \\
& \quad \leq C^{\prime}(b) \epsilon_{k^{*}} .
\end{aligned}
$$

(2) We now show that $\tilde{P}_{k^{*}, k} \eta_{k}$ is close to $P_{v_{k}} \eta_{k}$. By Lemmas 3.16 and 4.1,

$$
\begin{aligned}
\| \tilde{P}_{k^{*}, k} \eta_{k} & -P_{v_{k}} \eta_{k} \|_{v_{k}, p, 1} \\
& \leq C(b)\left(\left\|D_{v_{k}} \tilde{P}_{k^{*}, k} \eta_{k}-\eta_{k}\right\|_{v_{k}, p}+\left\|\pi_{v_{k},-} \tilde{P}_{k^{*}, k} \eta_{k}\right\|_{v_{k}, p, 1}\right) \\
& \leq C(b)\left(\left\|D_{v_{k}} \tilde{P}_{k^{*}, k} \eta_{k}-\eta_{k}\right\|_{v_{k}, p}+\left|v_{k}-v^{*}\right|\left\|\eta_{k}\right\|_{v_{k}, p}\right) .
\end{aligned}
$$

Since $D_{v^{*}} P_{v^{*}} \tilde{\eta}_{k^{*}, k}=\tilde{\eta}_{k^{*}, k}$ and $q_{\tilde{v}_{k}}$ is holomorphic outside of the annuli $A_{h, \delta_{k^{*}, k}}$

$$
D_{v_{k}} \tilde{P}_{k^{*}, k} \eta_{k}=\eta_{k} \quad \text { on } \Sigma_{v_{k}}-\bigcup_{h \in H} A_{h, \delta_{k^{*}, k}}
$$

By equation (4.6),

$$
\begin{aligned}
\left\|D_{v_{k}} \tilde{P}_{k^{*}, k} \eta_{k}\right\|_{v_{k}, L^{p}\left(A_{h, \delta_{k^{*}, k}}\right)} & \leq C(b)\left\|\tilde{P}_{k^{*}, k} \eta_{k}\right\|_{v_{k}, L_{1}^{p}\left(A_{h, \delta_{k^{*}, k}}\right)} \\
& \leq C^{\prime}(b) \epsilon_{k^{*}} .
\end{aligned}
$$

Thus, from equations (4.7)-(4.9) and the second assumption of (4.4), we conclude that for all $k>N_{k^{*}}$

$$
\left\|\tilde{P}_{k^{*}, k} \eta_{k}-P_{v_{k}} \eta_{k}\right\|_{v_{k}, p, 1} \leq C(b) \epsilon_{k^{*}}\left(1+\left\|\eta^{*}\right\|_{v^{*}, p}\right) .
$$

Since $\left\|d q_{\tilde{v}_{k}}^{-1}\right\|_{C^{0}} \leq C(b)$ on $\Sigma_{\delta_{k^{*}}^{*}}^{*}$, by equations (4.5), (4.6), and (4.10),

$$
\begin{gathered}
\left\|q_{\tilde{v}_{k}}^{-1 *} P_{v_{k}} \eta_{k}-P_{v^{*}} \eta^{*}\right\|_{v^{*}, L_{1}^{p}\left(\Sigma_{\delta_{k^{*}}^{*}}^{*}\right)} \leq C(b) \epsilon_{k^{*}}\left(1+\left\|\eta^{*}\right\|_{v^{*}, p}\right) \\
\left\|P_{v_{k}} \eta_{k}\right\|_{v_{k}, L_{1}^{p}\left(A_{\left.h, \delta_{k^{*}, k}\right)}\right.} \leq C(b) \epsilon_{k^{*}}\left(1+\left\|\eta^{*}\right\|_{v^{*}, p}\right) \quad \forall h \in H .
\end{gathered}
$$

By equations (4.11) and (4.12), $P_{v_{k}} \eta_{k}$ converges to $P_{v^{*}} \eta^{*}$.

Lemma 4.3. There exist $\tilde{C}, \delta \in C^{\infty}\left(\mathcal{M}_{\mathcal{T}}^{(0)} ; \mathbb{R}^{+}\right)$such that for all $v^{*} \in F^{(H)} \mathcal{T}_{\delta}$ and $h \in H$,

$$
\left\|P_{v^{*}} \bar{\partial} u_{v^{*}}\right\|_{g_{v^{*}}, C^{1}\left(A_{h, \delta\left(b_{v^{*}}\right)}^{*}\right)} \leq \tilde{C}\left(b_{v^{*}}\right)
$$

Proof. For each $h \in H$, this lemma is obtained by pasting

$$
\left.P_{v^{*}} \bar{\partial} u_{v^{*}}\right|_{A_{h, \delta\left(b_{v^{*}}^{*}\right)} \cap \Sigma_{v^{*}, \iota_{h}}} \quad \text { and }\left.\quad P_{v^{*}} \bar{\partial} u_{v^{*}}\right|_{A_{h, \delta\left(b_{v^{*}}\right)} \cap \Sigma_{v^{*}, h}}
$$


onto $\Sigma_{b_{v^{*}, \iota_{h}}}$ and $\Sigma_{b_{v^{*}, h}}$ via a cutoff function. We then use the usual elliptic estimates and Sobolev inequalities on $\Sigma_{b_{v^{*}, \iota_{h}}}$ and $\Sigma_{b_{v^{*}, h}}$ along with

$$
\left\|P_{v^{*}} \bar{\partial} u_{v^{*}}\right\|_{v^{*}, p, 1} \leq C\left(b_{v^{*}}\right)\left|v^{*}\right|^{\frac{1}{p}} .
$$

The bound obtained in this way is actually $C\left(b_{v^{*}}\right)\left|v^{*}\right|^{\frac{1}{p}}$.

Corollary 4.4. There exist $C, \delta \in C^{\infty}\left(\mathcal{M}_{\mathcal{T}}^{(0)} ; \mathbb{R}^{+}\right)$such that for any sequence $v_{k} \in F^{(\emptyset)} \mathcal{T}_{\delta}$ converging to $v^{*} \in F^{(H)} \mathcal{T}_{\delta}$ as above,

$$
\begin{aligned}
& \left\|q_{\tilde{v}_{k}}^{-1 *} \eta_{v_{k}}-\eta_{v^{*}}\right\|_{v^{*}, L^{p}\left(\Sigma_{2\left|v_{k}-v^{*}\right|^{1 / 2}}^{*}\right)} \leq C(b)\left|v_{k}-v^{*}\right|^{\frac{1}{p}} \\
& \left\|\eta_{v_{k}}\right\|_{v_{k}, L^{p}\left(A_{h, 2\left|v_{k}-v^{*}\right|^{1 / 2}, k}\right)} \leq C(b)\left|v_{k}-v^{*}\right|^{\frac{1}{p}} \quad \forall h \in H .
\end{aligned}
$$

Proof. We put

$$
\delta_{k}=2\left|v_{k}-v^{*}\right|^{\frac{1}{2}} \quad \text { and } \quad \epsilon_{k}=\left(2\left\|\beta^{\prime}\right\|_{C^{0}}+\tilde{C}(b)\right)\left|v_{k}-v^{*}\right|^{\frac{1}{p}},
$$

where $\tilde{C}$ is the function given by Lemma 4.3 . Let

$$
\begin{array}{lll}
\eta^{(0)}=-\bar{\partial} u_{v^{*}}, & \eta^{(m+1)}=-\bar{\partial} u_{v^{*}}-N_{v^{*}} P_{v^{*}} \eta^{(m)} & m \geq 0 ; \\
\eta_{k}^{(0)}=-\bar{\partial} u_{v_{k}}, & \eta_{k}^{(m+1)}=-\bar{\partial} u_{v_{k}}-N_{v_{k}} P_{v_{k}} \eta_{k}^{(m)} & m \geq 0 .
\end{array}
$$

By Lemma 4.3 and the explicit description of $\bar{\partial} q_{v_{k}}$ in Lemma $2.2, \epsilon_{k}, \delta_{k}$, $\eta^{(0)}$, and $\eta_{k}^{(0)}$ satisfy (4.3) and (4.4). Suppose $\epsilon_{k}^{(m)}$ is such that $\epsilon_{k}^{(m)}, \delta_{k}$, $\eta^{(m)}$, and $\eta_{k}^{(m)}$ satisfy (4.3) and (4.4). Since the map $q_{\tilde{v}_{k}}$ is holomorphic on $q_{\tilde{v}_{k}}^{-1}\left(\sum_{\delta_{k}}^{*}\right)$, by $(4.11),(4.12)$, the estimates in the proof of Lemma 3.18 and the derivation of equation (3.11) in $[\mathbf{Z 1}]$,

$$
\begin{gathered}
\left\|q_{v_{k}}^{-1 *} N_{v_{k}} P_{v_{k}} \eta_{k}^{(m)}-N_{v^{*}} P_{v^{*}} \eta^{(m)}\right\|_{v^{*}, L^{p}\left(\Sigma_{\delta_{k}}^{*}\right)} \\
=\left\|N_{v^{*}} q_{v_{k}}^{-1 *} P_{v_{k}} \eta_{k}^{(m)}-N_{v^{*}} P_{v^{*}} \eta^{(m)}\right\|_{v^{*}, L^{p}\left(\Sigma_{\delta_{k}}^{*}\right)} \\
\leq C(b)\left(\left\|q_{v_{k}}^{-1 *} P_{v_{k}} \eta_{k}^{(m)}\right\|_{v^{*}, L^{p}\left(\Sigma_{\delta_{k}}^{*}\right)}+\left\|P_{v_{k}} \eta_{k}^{(m)}\right\|_{v^{*}, L^{p}\left(\Sigma_{\delta_{k}}^{*}\right)}\right) \\
\quad \times\left\|q_{v_{k}}^{-1 *} P_{v_{k}} \eta_{k}^{(m)}-P_{v^{*}} \eta^{(m)}\right\|_{v^{*}, L^{p}\left(\Sigma_{\delta_{k}}^{*}\right)} \\
\leq C^{\prime}(b)\left(\epsilon_{k^{*}}^{(m)}+\left|v_{k}\right|^{\frac{1}{p}}\right) \epsilon_{k^{*}}^{(m)} ; \\
\left\|N_{v_{k}} P_{v_{k}} \eta_{k}^{(m)}\right\|_{v_{k}, L^{p}\left(A_{h, \delta_{k}, k}\right)} \leq C(b)\left|v_{k}\right|^{\frac{1}{p}}\left\|P_{v_{k}} \eta_{k}^{(m)}\right\|_{v_{k}, L_{1}^{p}\left(A_{h, \delta_{k}, k}\right)} \\
\leq C^{\prime}(b)\left|v_{k}\right|^{\frac{1}{p}} \epsilon_{k^{*}}^{(m)} .
\end{gathered}
$$

Thus, we can take

$$
\epsilon_{k}^{(m+1)}=\epsilon_{k}^{(m)}+C^{\prime}(b)\left(\epsilon_{k^{*}}^{(m)}+\left|v_{k}\right|^{\frac{1}{p}}\right) \epsilon_{k^{*}}^{(m)} .
$$


This sequence is bounded as long as $\left|v_{k}\right|^{\frac{1}{p}}$ is sufficiently small (depending only on $b$ ). Since $\eta_{v^{*}}$ is the limit in the $\left(v^{*}, p\right)$-norm of the sequence $\eta^{(m)}$ and $\eta_{v_{k}}$ is the limit in the $\left(v_{k}, p\right)$-norm of the sequence $\eta_{k}^{(m)}$, the claim follows.

Corollary 4.5. If $\mathcal{T}$ is a simple regular bubble type, there exist $\mathbb{R}^{+}$-valued smooth functions $\delta, C$ on $\mathcal{M}_{\mathcal{T}}$ such that for any sequence $\left\{v_{k} \in F^{\emptyset} \mathcal{T}_{\delta}\right\}$ converging to $v^{*} \in F^{H} \mathcal{T}_{\delta}, \tilde{\gamma}\left(v_{k}\right)$ converges to $\tilde{\gamma}\left(v^{*}\right)$ with respect to the Gromov topology. Furthermore,

$$
\begin{array}{ll}
d_{V}\left(\operatorname{ev}\left(\tilde{\gamma}\left(v^{*}\right)\right), \operatorname{ev}\left(\tilde{\gamma}\left(v_{k}\right)\right)\right) \leq C\left(b_{v^{*}}\right)\left|v_{k}-v^{*}\right|^{\frac{1}{p}} & \text { if } S=S^{2} ; \\
d_{V}\left(\operatorname{ev}_{l}\left(\tilde{\gamma}\left(v^{*}\right)\right), \operatorname{ev}_{l}\left(\tilde{\gamma}\left(v_{k}\right)\right)\right) \leq C\left(b_{v^{*}}\right)\left|v_{k}-v^{*}\right|^{\frac{1}{p}} & \forall l \in M ; \\
\left|\Psi_{\langle\mathcal{T}\rangle, \hat{0}}\left(\tilde{\gamma}\left(v_{k}\right)\right)-\Psi_{\mathcal{T}\left(v^{*}\right), \hat{0}}\left(\tilde{\gamma}\left(v^{*}\right)\right)\right| \leq C\left(b_{v^{*}}\right)\left|v_{k}-v^{*}\right|^{\frac{1}{p}} & \text { if } S=S^{2} .
\end{array}
$$

Proof. It is sufficient to consider the case $\pi_{h}\left(v_{k}\right)=\pi_{h}\left(v^{*}\right)$ for all $h \notin H$ if $v^{*} \in F^{(H)} \mathcal{T}_{\delta}$. In such a case, $q_{\tilde{v}_{k}}$ maps the marked points of $\Sigma_{v_{k}}$ to the marked points of $\Sigma_{v^{*}}$ and $u_{v_{k}}=u_{v^{*}} \circ q_{v_{k}}$. By construction,

$$
\tilde{u}_{v_{k}}=\exp _{b_{v^{*}, u_{v_{k}}}} P_{v_{k}} \eta_{v_{k}}, \quad \tilde{u}_{v_{k}}=\exp _{b_{v^{*}, u_{v^{*}}}} P_{v^{*}} \eta_{v^{*}} .
$$

By Corollary 4.5 and the proof of Lemma 4.2 ,

$$
\begin{gathered}
\left\|q_{\tilde{v}_{k}}^{-1 *} P_{v_{k}} \eta_{v_{k}}-P_{v^{*}} \eta_{v^{*}}\right\|_{v^{*}, L_{1}^{p}\left(\Sigma_{\left.2\left|v_{k}-v^{*}\right|\right|^{1 / 2}}^{*}\right)} \leq C\left(b_{v^{*}}\right)\left|v_{k}-v^{*}\right|^{\frac{1}{p}} \\
\left\|P_{v_{k}} \eta_{v_{k}}\right\|_{v_{k}, L_{1}^{p}\left(A_{h, 2\left|v_{k}-v^{*}\right|^{1 / 2}, k}\right)} \leq C\left(b_{v^{*}}\right)\left|v_{k}-v^{*}\right|^{\frac{1}{p}} \quad \forall h \in H .
\end{gathered}
$$

Let $\zeta_{k} \in \Gamma\left(\Sigma^{*} ; \tilde{u}_{v^{*}}\right)$ be given by

$$
\exp _{b_{v^{*}}, \tilde{u}_{v^{*}}} \zeta_{k}=\tilde{u}_{v_{k}} \circ q_{\tilde{v}_{k}}, \quad\left\|\zeta_{k}\right\|_{C^{0}}<\operatorname{inj} g_{V, b_{v^{*}}} .
$$

By equation (4.13) and the proof of (2) of Lemma 3.5,

$$
\begin{aligned}
\left\|\zeta_{k}\right\|_{C^{0}\left(\Sigma_{\left.2\left|v_{k}-v^{*}\right|\right|^{1 / 2}}^{*}\right)} & \leq C\left(b_{v^{*}}\right)\left\|\zeta_{k}\right\|_{v^{*}, L_{1}^{p}\left(\Sigma_{2\left|v_{k}-v^{*}\right|^{1 / 2}}\right)} \\
& \leq C^{\prime}\left(b_{v^{*}}\right)\left|v_{k}-v^{*}\right|^{\frac{1}{p}}
\end{aligned}
$$

On the other hand, by (4.14) and by the same argument as in (3) of the proof of Lemma 5.12, the variations of $P_{v^{*}} \eta_{v^{*}}$ on $A_{h, 2\left|v_{k}-v^{*}\right|^{1 / 2}}^{*}$ and $P_{v_{k}} \eta_{v_{k}}$ on $A_{h, 2\left|v_{k}-v^{*}\right|^{1 / 2}, k}^{*}$ are bounded $C\left(b_{v}\right)\left|v_{k}-v^{*}\right|^{\frac{1}{p}}$. This can be seen from equation (5.33); observe that an argument similar to the proof Lemma 4.3 shows that we can take $\delta$ to be any small number bigger than $2\left|v_{k}-v^{*}\right|^{\frac{1}{2}}$. Equation (4.15) and the small variation on the annuli imply that

$$
\sup _{z \in \Sigma_{v_{k}}} d_{V}\left(\tilde{u}_{v^{*}}\left(q_{\tilde{v}_{k}}(z)\right), \tilde{u}_{v_{k}}(z)\right) \leq C\left(b_{v^{*}}\right)\left|v_{k}-v^{*}\right|^{\frac{1}{p}} .
$$

It follows that $\tilde{\gamma}_{\mathcal{T}}\left(v_{k}\right)$ converges to $\tilde{\gamma}_{\mathcal{T}}\left(v^{*}\right)$ in the Gromov topology. The estimate on the evaluation maps is immediate from the above bound. The 
last estimate follows from equations (4.13) and (4.14), along with a Sobolev estimate on a neighborhood of $\infty \in \Sigma_{v^{*}, \hat{0}}$ which implies that the $C^{1}$-norm of $\zeta_{k}$ there is bounded by $C\left(b_{v^{*}}\right)\left|v_{k}-v^{*}\right|^{\frac{1}{p}}$.

4.2. Injectivity of the Gluing Map. The goal of this subsection is to prove that the gluing maps of (3.15) and (3.16) are injective, as long as $\delta \in C^{\infty}\left(\mathcal{M}_{\mathcal{T}}^{(0)} ; \mathbb{R}^{+}\right)$is sufficiently small. We start by showing local injectivity on the subspaces $F^{H} \mathcal{T}_{\delta}$ of $F \mathcal{T}_{\delta}$, where $H$ is a subset of $\hat{I}$.

If $\mathcal{T}$ is regular, we are only interested in the case $t=0$. If $\mathcal{T}$ is semiregular, we only consider the case $H=\emptyset$. We use the same notation as in Subsection 3.4. If $\|\varpi\|_{v}$ is sufficiently small, define $\tilde{\zeta}_{\varpi, t \nu} \in \Gamma\left(\tilde{u}_{v, t \nu}\right)$ by

$$
\exp _{b_{v}, \tilde{u}_{v, t \nu}} \tilde{\zeta}_{\varpi, t \nu}=u_{\varpi, t \nu}, \quad\left\|\tilde{\zeta}_{\varpi, t \nu}\right\|_{b_{v}, C^{0}}<\operatorname{inj} g_{V, b} .
$$

Lemma 4.6. There exist $\delta, C \in C^{\infty}\left(\mathcal{M}_{\mathcal{T}}^{(0)} ; \mathbb{R}^{+}\right)$such that for all $v \in F^{(H)} \mathcal{T}_{\delta}$, where $H=\emptyset$ if $\mathcal{T}$ is semiregular, and $\varpi \in \tilde{T}_{v} F^{H} \mathcal{T}_{\delta\left(b_{v}\right)}$,

(1) $\left\|S_{\varpi} N_{\varpi, t \nu} R_{\varpi} \xi-N_{v, t \nu} \xi\right\|_{v, p} \leq C\left(b_{v}\right)\|\varpi\|_{v}\|\xi\|_{v, p, 1}^{2}$ for all $\xi \in \Gamma\left(u_{v}\right)$ with $\|\xi\|_{v, p, 1} \leq \delta\left(b_{v}\right)$ and $t \in[0,1]$;

(2) $\left\|S_{\varpi} \tilde{\pi}_{\varpi, \pm} R_{\varpi} \xi-\tilde{\pi}_{v, \pm} \xi\right\|_{v, p, 1} \leq C\left(b_{v}\right)\|\varpi\|_{v}\|\xi\|_{v, p, 1}$ for all $\xi \in \Gamma\left(u_{v}\right)$;

(3) $\left\|S_{\varpi} P_{\varpi} R_{\varpi} \eta-P_{v} \eta\right\|_{v, p, 1} \leq C\left(b_{v}\right)\|\varpi\|_{v}\|\eta\|_{v, p}$ for all $\xi \in \Gamma^{0,1}\left(u_{v}\right)$.

Proof. Claim (1) follows from (2) of Lemma 3.6 and Riemannian geometry estimates such as in [Z1]. Claim (2) is a consequence of (5) of Lemma 3.6 and (b) of Definition 3.11. Finally, (3) is obtained from (1), (2), Definitions 3.11 and 3.13 , and Lemmas 3.6 and 3.16 as follows. Writing $\triangle_{\varpi} P$ for $S_{\varpi} P_{\varpi} R_{\varpi}-P_{v}$, etc.,

$$
\begin{aligned}
& \triangle_{\varpi} P=P_{v} \pi_{v,+}^{0,1} D_{v} \tilde{\pi}_{v,+} \triangle_{\varpi} P+\tilde{\pi}_{v,-} S_{\varpi} P_{\varpi} R_{\varpi} \\
& =P_{v} \pi_{v,+}^{0,1} D_{v} \triangle_{\varpi} P-\left(P_{v} \pi_{v,+}^{0,1} D_{v}-1\right) \triangle_{\varpi} \tilde{\pi}_{\cdot,+} S_{\varpi} P_{\varpi} R_{\varpi} \\
& =P_{v} \pi_{v,+}^{0,1} \triangle_{\varpi} \tilde{\pi}_{\cdot,+}^{0,1}-\left(P_{v} \pi_{v,+}^{0,1} \triangle_{\varpi} D+\left(P_{v} \pi_{v,+}^{0,1} D_{v}-1\right) \triangle_{\varpi} \tilde{\pi}_{\cdot,+}\right) S_{\varpi} P_{\varpi} R_{\varpi} \\
& =-P_{v} \triangle_{\varpi} \pi_{\cdot,+}^{0,1} S_{\varpi} \tilde{\pi}_{\varpi,+}^{0,1} R_{\varpi} \\
& \quad-\left(P_{v} \pi_{v,+}^{0,1} \triangle_{\varpi} D+\left(P_{v} \pi_{v,+}^{0,1} D_{v}-1\right) \triangle_{\varpi} \tilde{\pi}_{\varpi,+} S_{\varpi} P_{\varpi} R_{\varpi}\right) .
\end{aligned}
$$

Corollary 4.7. There exist $\delta, C \in C^{\infty}\left(\mathcal{M}_{\mathcal{T}}^{(0)} ; \mathbb{R}^{+}\right)$such that for all

$$
t \in\left[0 ; \delta\left(b_{v}\right)\right], \quad v \in F^{(H)} \mathcal{T}_{\delta},
$$

where $H=\emptyset$ if $\mathcal{T}$ is semiregular, and $\varpi \in \tilde{T}_{v} F^{H} \mathcal{T}_{\delta\left(b_{v}\right)}$,

$$
\begin{aligned}
C\left(b_{v}\right)^{-1}\|\varpi\|_{v} & \leq\left\|\tilde{\zeta}_{\varpi, t \nu}\right\|_{v, p, 1}+\sum_{h \in H}\left|w_{h}(\varpi)\right|_{g_{v}}+\sum_{l \in M}\left|w_{l}(\varpi)\right|_{g_{v}} \\
& \leq C\left(b_{v}\right)\|\varpi\|_{v} .
\end{aligned}
$$


Furthermore, $\left\|S_{\varpi} \xi_{\varpi, t \nu}-\xi_{v, t \nu}\right\|_{v, p, 1} \leq C\left(b_{v}\right)\left(t+|v|^{\frac{1}{p}}\right)\|\varpi\|_{v}$.

Proof. The first claim of the lemma is immediate from the second and (1) of Lemma 3.6. On the other hand, by construction in Subsection 3.6,

$$
\xi_{\varpi, t \nu}=t P_{\varpi} \nu-P_{\varpi} \bar{\partial} u_{\varpi}-P_{\varpi} N_{\varpi, t \nu} \xi_{\varpi, t \nu}
$$

Thus, if $t$ and $|v|$ are sufficiently small (depending on $b_{v}$ ), the second claim follows from Lemmas 3.6, 4.6, Corollary 3.19, and equation (3.11).

Corollary 4.8. If $\mathcal{T}$ is a simple bubble type and $K$ is an open subset of $\mathcal{M}_{\mathcal{T}}$ with compact closure, there exists $\delta>0$ such that for any $t \in[0, \delta]$, the map

$$
\tilde{\gamma}_{\mathcal{T}, t \nu}:\left.F^{\emptyset} \mathcal{T}_{\delta}\right|_{K} \longrightarrow C_{(\lambda ; M)}^{\infty}(S ; V), \quad \tilde{\gamma}_{\mathcal{T}, t \nu}(v)=\tilde{b}_{t \nu}(v),
$$

is a differentiable embedding.

Proof. We first deduce from Corollary 4.7 that $\tilde{\gamma}_{\mathcal{T}, t \nu}$ is injective if $\delta$ is sufficiently small. Suppose not, i.e., there exist sequences $v_{k},\left.v_{k^{\prime}} \in F^{\emptyset} \mathcal{T}_{\delta}\right|_{K}$ such that

$$
v_{k} \longrightarrow b \in \bar{K}, \quad v_{k}^{\prime} \longrightarrow b^{\prime} \in \bar{K} \text {, and } \tilde{b}_{t \nu}\left(v_{k}\right)=\tilde{b}_{t \nu}\left(v_{k}^{\prime}\right) .
$$

It follows that $b=b^{\prime}$, after possibly modifying the sequence $\left\{v_{k^{\prime}}\right\}$ the action of an element of $\left(\mathcal{A}(\mathcal{T}) \ltimes G_{\mathcal{T}}\right)$. If for some $k, v_{k}^{\prime}=v_{k}\left(\varpi_{k}\right)$ with $\left\|\varpi_{k}\right\|_{v_{k}}$ sufficiently small, then by Corollary $4.7, v_{k}^{\prime}=v_{k}$. Otherwise, the difference between $q_{v_{k}}$ and $q_{v_{k}^{\prime}}$ is uniformly bounded below outside of the preimage of the zeroth component and the necks $A_{v_{k}, h}$. Thus, the bubble maps $b\left(v_{k}\right)$ and $b\left(v_{k}^{\prime}\right)$ are far apart unless $b$ has an automorphism. In the latter case, $v_{k}^{\prime}$ can be replaced by an equivalent element of $F^{\emptyset} \mathcal{T}_{\delta}$. In the former case, $\tilde{u}_{v_{k}}$ and $\tilde{u}_{v_{k}^{\prime}}$ cannot be the same because

$$
\begin{gathered}
\left\|P_{v_{k}} \eta_{v_{k}, t \nu}\right\|_{C^{0}} \leq C\left(t+\left|v_{k}\right|^{\frac{1}{p}}\right) \leq C^{\prime} \delta^{\frac{1}{p}} \quad \text { and } \\
\left\|P_{v_{k}^{\prime}} \eta_{v_{k}^{\prime}, t \nu}\right\|_{C^{0}} \leq C\left(t+\left|v_{k}^{\prime}\right|^{\frac{1}{p}}\right) \delta^{\frac{1}{p}} .
\end{gathered}
$$

Thus, $\tilde{\gamma}_{\mathcal{T}, t \nu}$ is injective on $\left.F^{\emptyset} \mathcal{T}_{\delta}\right|_{K}$ provided $\delta$ is sufficiently small (depending on $K)$. The smoothness of $\tilde{\gamma}_{\mathcal{T}, t \nu}$ follows from the smooth dependence of solutions of equation (3.12) on the parameters. Finally, the differential of $\tilde{\gamma}_{\mathcal{T}, t \nu}$ is nondegenerate by Corollary 4.7 .

Corollary 4.9. If $\mathcal{T}$ is regular, there exists $\delta \in C^{\infty}\left(\mathcal{M}_{\mathcal{T}} ; \mathbb{R}^{+}\right)$such that for all $m$, the map

$$
\tilde{\gamma}_{\mathcal{T}}: \bigcup_{|H|=m} F^{H} \mathcal{T}_{\delta} \longrightarrow \bigcup_{|H|=m} \mathcal{M}_{\mathcal{T}(H)}, \quad \tilde{\gamma}_{\mathcal{T}}(v)=\tilde{b}(v)
$$

is injective. 
Proof. The same argument as in the proof of Corollary 4.8 shows that map

$$
\tilde{\gamma}_{\mathcal{T}}: F^{H} \mathcal{T}_{\delta} \longrightarrow \mathcal{H}_{\mathcal{T}(H)}
$$

is an embedding if $\delta$ is sufficiently small. It remains to see that $\tilde{\gamma}_{\mathcal{T}}^{(0)}(v) \neq$ $g \cdot \tilde{\gamma}_{\mathcal{T}}^{(0)}\left(v^{\prime}\right)$ for any $g \in G_{\mathcal{T}(H)}$ whenever $[v] \neq\left[v^{\prime}\right]$. For each $v \in F^{(H)} \mathcal{T}_{\delta}$ and $i \in H$, we construct $\left(c_{i}(v), r_{i}(v)\right) \in \mathbb{C} \times \mathbb{R}$ such that

$$
(c(v), r(v)) \cdot \tilde{\gamma}_{\mathcal{T}}^{(0)}(v) \in \mathcal{M}_{\mathcal{T}(H)}^{(0)} .
$$

We define $c_{i}(v) \in \mathbb{C}$ and $r_{i}(v) \in \mathbb{R}$ by

$$
\begin{gathered}
\tilde{\Psi}\left(\left(c_{i}(v), 0\right) \cdot \tilde{u}_{v, i}\right)+\sum_{\iota_{h}(v)=i} d_{h}(\mathcal{T}(H))\left(x_{h}(v)+c_{i}(v)\right) \\
+\sum_{j_{l}(v)=i}\left(y_{l}(v)+c_{i}(v)\right)=0 \\
\Psi^{(3)}\left(\left(c_{i}(v), r_{i}(v)\right) \cdot \tilde{u}_{v, i}\right)+\sum_{\iota_{h}(v)=i} d_{h}(\mathcal{T}(H)) \beta\left(\left(1+r_{i}(v)\right)\left|x_{h}(v)+c_{i}(v)\right|\right) \\
+\sum_{j_{l}(v)=i} \beta\left(\left(1+r_{i}(v)\right)\left|y_{l}(v)+c_{i}(v)\right|\right)=\frac{1}{2} .
\end{gathered}
$$

Since the metric $g_{v, i}$ for $i>0$ agrees with the standard metric on $S^{2}$ on a neighborhood of the south pole and $\Psi_{\mathcal{T}, i}\left(b_{v}\right)=0$, by Corollary 4.7 for any $\varpi \in T_{v} F^{H} \mathcal{T}$ with $\|\varpi\|_{v}$ sufficiently small,

$$
\begin{aligned}
& \left|c_{i}(\varpi)-c_{i}(v)\right| \leq C\left(b_{v}\right)|v|^{\frac{1}{p}}\|\varpi\|_{v}, \\
& \left|r_{i}(\varpi)-r_{i}(v)\right| \leq C\left(b_{v}\right)|v|^{\frac{1}{p}}\|\varpi\|_{v} .
\end{aligned}
$$

Let $\bar{b}(v)=(c(v), r(v)) \cdot \tilde{b}(v)$. Write

$$
\bar{b}(v)=\left(S, M, H \cup\{\hat{0}\} ; \bar{x}(v),(j(v), \bar{y}), \bar{u}_{v}\right) .
$$

If $\|\varpi\|_{v}$ is sufficiently small, define $\bar{\zeta}_{\varpi} \in \Gamma\left(\bar{u}_{v}\right)$ by

$$
\exp _{b_{v}, \bar{u}_{v}} \bar{\zeta}_{\varpi}=u_{\varpi}, \quad\left\|\bar{\zeta}_{\varpi}\right\|_{b_{v}, C^{0}}<\operatorname{inj} g_{V, b_{v}} .
$$

Similarly, for $h \in H$ and $l \in M$, define $\bar{w}_{h}(\varpi) \in T_{\bar{x}_{h}(v)} \Sigma_{v, \iota_{h}(v)}$ and $\bar{w}_{l}(\varpi) \in$ $T_{\bar{y}_{l}} \Sigma_{v, j_{l}(v)}$ by

$$
\begin{aligned}
\exp _{g_{v}, \bar{x}_{h}(v)} \bar{w}_{h}(\varpi)=\bar{x}_{h}(\varpi), & \left|\bar{w}_{h}(\varpi)\right| \equiv\left|\bar{w}_{h}(\varpi)\right|_{g_{v}}<\operatorname{inj}\left(g_{v}, x_{h}(v)\right) ; \\
\exp _{g_{v}, \bar{y}_{l}(v)} \bar{w}_{l}(\varpi)=\bar{y}_{l}(\varpi), & \left|\bar{w}_{l}(\varpi)\right| \equiv\left|\bar{w}_{l}(\varpi)\right|_{g_{v}}<\operatorname{inj}\left(g_{v}, y_{l}(v)\right) .
\end{aligned}
$$

Then by equation (4.16) and Corollary 4.7,

$$
\begin{aligned}
C^{\prime \prime}\left(b_{v}\right)^{-1}\|\varpi\|_{v} & \leq\left\|\bar{\zeta}_{\varpi}\right\|_{v, p, 1}+\sum_{h \in H}\left|\bar{w}_{h}(\varpi)\right|+\sum_{l \in M}\left|\bar{w}_{l}(\varpi)\right| \\
& \leq C\left(b_{v}\right)\|\varpi\|_{v} .
\end{aligned}
$$


It follows that the map

$$
F^{H} \mathcal{T}_{\delta} \longrightarrow \mathcal{M}_{\mathcal{T}(H)}^{(0)}, \quad v \longrightarrow \bar{b}(v),
$$

is a local embedding. By the same argument as in the proof of Lemma 4.8, we can conclude that this map is injective as long as $\delta \in C^{\infty}\left(\mathcal{M}_{\mathcal{T}}^{(0)} ; \mathbb{R}^{+}\right)$is sufficiently small. Since this map is $G_{\mathcal{T}(H)^{-}}$equivariant by construction, it follows that the induced map on the quotient, i.e., the map of Corollary 4.9, is injective.

Corollary 4.10. If $S=S^{2}$, there exists $\delta \in C^{\infty}\left(\mathcal{M}_{\mathcal{T}} ; \mathbb{R}^{+}\right)$such that the map

$$
\tilde{\gamma}_{\mathcal{T}}:\left.F \mathcal{T}_{\delta}\right|_{\mathcal{M}_{\mathcal{T}}} \longrightarrow \overline{\mathcal{M}}_{\langle\mathcal{T}\rangle}
$$

is injective. Furthermore, the restriction

$$
\tilde{\gamma}_{\mathcal{T}}:\left.F^{\emptyset} \mathcal{T}_{\delta}\right|_{\mathcal{M}_{\mathcal{T}}} \longrightarrow \mathcal{M}_{\langle\mathcal{T}\rangle}
$$

is a differentiable embedding.

In order to adjust the gluing procedure in the presence of constraints, below we state the analogue of Corollary 4.7 for $\varpi \in \mathcal{K}_{b_{v}} \mathcal{T} \subset T_{v} F^{\emptyset} \mathcal{T}$. It is obtained in the same way as Corollary 4.7, except the analogue of Lemma 4.6 would make use of Lemma 3.8, instead of Lemma 3.6, and of (b-ii), instead of (b-i), of Definitions 3.11 and 3.13. We also use (3) of Lemma 3.5.

Corollary 4.11. There exist $\delta, C \in C^{\infty}\left(\mathcal{M}_{\mathcal{T}}^{(0)} ; \mathbb{R}^{+}\right)$such that for all $t \in$ $\left[0 ; \delta\left(b_{v}\right)\right], v \in F^{(\emptyset)} \mathcal{T}_{\delta}$, and $\varpi \in \mathcal{K}_{b_{v}} \mathcal{T}_{\delta\left(b_{v}\right)}$,

$$
C\left(b_{v}\right)^{-1}\|\varpi\| \leq\left\|\tilde{\zeta}_{\varpi, t \nu}\right\|_{v, p, 1}+\sum_{l \in M}\left|w_{l}(\varpi)\right|_{g_{v}} \leq C\left(b_{v}\right)\|\varpi\| .
$$

Furthermore, $\left\|S_{\varpi}^{\prime} \xi_{\varpi, t \nu}-\xi_{v, t \nu}\right\|_{v, C^{0}} \leq C\left(b_{v}\right)\left(t+|v|^{\frac{1}{p}}\right)\|\varpi\|$.

4.3. The Basic Gluing Map and the Space of Balanced Maps. Our next goal is to show that the gluing map of Subsection 3.6 is surjective in the appropriate sense. More precisely, if $\mathcal{T}$ is a regular bubble type, we show that the image of $\tilde{\gamma}_{\mathcal{T}}$ contains a neighborhood of $\mathcal{M}_{\mathcal{T}}$ in $\overline{\mathcal{M}}_{\langle\mathcal{T}\rangle}$. If $\mathcal{T}$ is a semiregular, we show that all elements in $\mathcal{M}_{\Sigma, t \nu, \lambda}$ that are close to any given compact subset of $\mathcal{M}_{\mathcal{T}}$ are in the image of the gluing map $\tilde{\gamma}_{\mathcal{T}, t \nu}$ if $t$ is sufficiently small. The major difficulty in doing this is the following. If $v \in F \mathcal{T}$, a small change in the singular points of $b_{v}$ may lead to a very large change in the map $u_{v}$. This is precisely the reason we used the norm $\|\varpi\|_{v}$ on $T_{v} F^{H} \mathcal{T}$ instead of just $\|\varpi\|$ in Subsection 3.4. In order to deal with this issue, we need Corollary 4.13, which is proved in this subsection. We continue to assume that $\mathcal{T}$ is a simple bubble type.

Recall that $\mathcal{H}_{\mathcal{T}}$ is the set of tuples $b=(S, M, I ; x,(j, y), u)$ such that $u_{\iota_{h}}\left(x_{h}\right)=u_{h}(\infty)$ for all $h \in \hat{I}$ and $\bar{\partial} u_{i}=0$ for all $i \in I$. Furthermore, $\mathcal{M}_{\mathcal{T}}^{(0)}$ is 
the subset of $\mathcal{H}_{\mathcal{T}}$ consisting of the tuples $b$ such that $\Psi_{\mathcal{T}, h}(b)=0$ for all $h \in \hat{I}$. It is convenient to make the following definitions. If $H$ is a subset of $\hat{I}$ and $\epsilon \geq 0$, let

$$
\begin{aligned}
\mathcal{M}_{\mathcal{T}, \epsilon}^{(H)}=\{b=(S, M, I ; x,(j, y), u): & \bar{\partial} u_{i}=0 \forall i \in I ; \\
& d_{V}\left(u_{\iota_{h}}\left(x_{h}\right), u_{h}(\infty)\right) \leq \epsilon \forall h \in \hat{I} ; \\
& \left.\left|\Psi_{\mathcal{T}, h}(b)\right| \leq \epsilon \forall h \in \hat{I}-H\right\} .
\end{aligned}
$$

Lemma 4.12. There exist $\delta, C \in C^{\infty}\left(\mathcal{M}_{\mathcal{T}}^{(0)} ; \mathbb{R}^{+}\right)$with the following property. Suppose $b^{*} \in \mathcal{M}_{\mathcal{T}}^{(0)}, \epsilon<\delta\left(b^{*}\right), b \in \mathcal{M}_{\mathcal{T}, \epsilon}^{(H)}$ is such that $d\left(b^{*}, b\right) \leq \delta\left(b^{*}\right)$, and $v=\left(b, v_{\hat{I}}\right) \in F_{b}^{(H)} \mathcal{T}_{\delta\left(b^{*}\right)}$. Then there exist

$$
\tilde{b} \in \mathcal{M}_{\mathcal{T}, \epsilon^{2}}^{(H)} \quad \text { and } \quad \tilde{v}=\left(\tilde{b}, \tilde{v}_{\hat{I}}\right) \in F_{\tilde{b}}^{(H)} \mathcal{T}
$$

such that

(1) $d(b, \tilde{b}) \leq C\left(b^{*}\right) \epsilon$ and $\left|\tilde{v}_{h}-v_{h}\right|_{b} \leq C\left(b^{*}\right) \epsilon\left|v_{h}\right|_{b}$ for all $h \in \hat{I}$;

(2) if $q_{v}(z) \in \Sigma_{\mathcal{T}, i}, r_{b, h}\left(q_{v} z\right) \geq 2\left|v_{h}\right|^{\frac{1}{2}}$ for all $h \in \hat{I}-H$ such that $\iota_{h}=i$ and $\left|q_{S}^{-1}\left(q_{v} z\right)\right| \geq 2\left|v_{i}\right|^{\frac{1}{2}}$ if $i \in \hat{I}-H$, then $d_{b}\left(q_{v}(z), q_{\tilde{v}}(z)\right) \leq \delta\left(b^{*}\right) \epsilon$.

Proof. (1) Let $b=(S, M, I ; x,(j, y), u)$. If $\delta$ is sufficiently small, by Proposition 3.3 , we can choose $\xi_{i} \in \Gamma\left(u_{i}\right)$ such that $\left\|\xi_{i}\right\|_{g_{b, i}, C^{1}} \leq C\left(b^{*}\right) \epsilon$ and

$$
b^{\prime} \equiv\left(S, M, I ; x,(j, y), u^{\prime}\right) \in \mathcal{H}_{\mathcal{T}},
$$

where $u_{i}^{\prime}=\exp _{u_{i}} \xi_{i}$. The $C^{1}$-bound on $\xi_{i}$ and the assumption $b \in \mathcal{M}_{\mathcal{T}, \epsilon}^{(H)}$ imply that $\left|\Psi_{\mathcal{T}, h}\left(b^{\prime}\right)\right| \leq C^{\prime}\left(b^{*}\right) \epsilon$ for all $h \in \hat{I}-H$.

(2) We now define $\tilde{b}^{\prime} \equiv\left(S, M, I ; \tilde{x}^{\prime},\left(j, \tilde{y}^{\prime}\right), \tilde{u}^{\prime}\right) \in \mathcal{H}_{\mathcal{T}}$ and $\tilde{v}^{\prime}=\left(\tilde{b}^{\prime}, \tilde{v}_{\hat{I}}^{\prime}\right)$ as follows. Suppose $i^{*} \in I$ and for all $i \in \hat{I}$ with $i>i^{*}, h \in \hat{I}$ with $\iota_{h}=i$, and $l \in M$ with $j_{l}=i$, we have constructed

(i) $\left(c_{i}, r_{i}\right) \in \mathbb{C} \times \mathbb{R}$ such that $\left|\left(c_{i}, r_{i}\right)\right| \leq C\left(b^{*}\right) \epsilon$;

(ii) $\tilde{x}_{h}^{\prime}, \tilde{y}_{l}^{\prime} \in \Sigma_{\mathcal{T}, i}$ such that $\left|r_{b, h}\left(\tilde{x}_{h}^{\prime}\right)\right| \leq C\left(b^{*}\right) \epsilon$ and $\left|\phi_{y_{l}} \tilde{y}_{l}^{\prime}\right| \leq C\left(b^{*}\right) \epsilon$;

(iii) $\tilde{v}_{h}^{\prime} \in \mathbb{C}$ such that $\left|\tilde{v}_{h}^{\prime}-v_{h}\right| \leq C\left(b^{*}\right) \epsilon\left|v_{h}\right|$;

(iv) if $x_{i} \in S^{2}, \bar{x}_{i} \in S^{2}$, such that $\left|r_{b, i}\left(\bar{x}_{i}\right)\right| \leq C\left(b^{*}\right) \epsilon\left|v_{i}\right|$;

(v) if $x_{i} \in S^{2}, \bar{v}_{i} \in \mathbb{C}$ such that $\left|\bar{v}_{i}-v_{i}\right| \leq C\left(b^{*}\right) \epsilon\left|v_{i}\right|$, such that

(I1) if $i \notin H, \Psi_{\mathcal{T}, i}\left(\tilde{b}^{\prime}\right)=0$ where $\tilde{u}_{i}^{\prime}=\left(c_{i}, r_{i}\right) \cdot u_{i}^{\prime}$;

(I2) if $\Sigma_{\mathcal{T}, \iota_{i}}=S^{2}, z \in \Sigma_{\mathcal{T}, \iota_{i}}$, and $\left|\phi_{\bar{x}_{h}} q_{i,\left(x_{i}, v_{i}\right)}(z)\right| \leq \frac{2}{3}\left|\bar{v}_{h}\right|^{\frac{1}{2}}$ for some $h \in \hat{I}-H$, then

$$
\begin{gathered}
\left|\phi_{\tilde{x}_{h}^{\prime}} q_{i,\left(\bar{x}_{i}, \bar{v}_{i}\right)}(z)\right| \leq\left|\tilde{v}_{h}^{\prime}\right|^{\frac{1}{2}} \quad \text { and } \\
q_{h,\left(\tilde{x}_{h}^{\prime}, \tilde{v}_{h}^{\prime}\right)}\left(q_{i,\left(\bar{x}_{i}, \bar{v}_{i}\right)}(z)\right)=q_{h,\left(\bar{x}_{h}, \bar{v}_{h}\right)}\left(q_{i,\left(x_{i}, v_{i}\right)}(z)\right),
\end{gathered}
$$


where $q_{i,\left(x_{i}, v_{i}\right)}$, etc., are the maps defined in Subsection 2.2.

Note that while we have not defined $\tilde{b}^{\prime}$ completely yet, (I1) is still a welldefined statement. The function $\Psi_{\mathcal{T}, i}$ depends only the $i$ th bubble component of $\tilde{b}^{\prime}$, which has already been constructed by the induction assumptions.

If $i^{*} \in H$, we take $c_{i^{*}}=0$ and $r_{i^{*}}=0$. If $i^{*} \in \hat{I}-H$, let $\left(c_{i^{*}}, r_{i^{*}}\right) \in \mathbb{C} \times \mathbb{R}$ be given by

$$
\begin{gathered}
\tilde{\Psi}\left(\left(c_{i^{*}}, 0\right) u_{i^{*}}^{\prime}\right)+\sum_{\iota_{h}=i^{*}} d_{h}(\mathcal{T})\left(\bar{x}_{h}+c_{i^{*}}\right)+\sum_{j_{l}=i^{*}}\left(y_{l}+c_{i^{*}}\right)=0 \\
\Psi^{(3)}\left(\left(c_{i^{*}}, r_{i^{*}}\right) u_{i^{*}}^{\prime}\right)+\sum_{\iota_{h}=i^{*}} d_{h}(\mathcal{T}) \beta\left(\left(1+r_{i^{*}}\right)\left|\bar{x}_{h}+c_{i^{*}}\right|\right) \\
+\sum_{j_{l}=i^{*}} \beta\left(\left(1+r_{i^{*}}\right)\left|y_{l}+c_{i^{*}}\right|\right)=\frac{1}{2} .
\end{gathered}
$$

If $\epsilon$ is sufficiently small, by the proof of Lemma 3.3 such $\left(c_{i^{*}}, r_{i^{*}}\right) \in \mathbb{C} \times \mathbb{R}$ exists and satisfies $\left|c_{i^{*}}\right|,\left|r_{i^{*}}\right| \leq C\left(b^{*}\right) \epsilon$. For all $h \in \hat{I}$ with $\iota_{h}=i^{*}$ and $l \in M$ with $j_{l}=i^{*}$, put

$$
\begin{gathered}
\tilde{x}_{h}^{\prime}=\left(1+r_{i^{*}}\right)\left(\bar{x}_{h}+c_{i^{*}}\right), \quad \tilde{v}_{h}^{\prime}=\left(1+r_{i^{*}}\right) \bar{v}_{h}, \quad \tilde{y}_{l}^{\prime}=\left(1+r_{i^{*}}\right)\left(y_{l}+c_{i^{*}}\right) ; \\
\bar{x}_{i^{*}}=x_{i^{*}}-c_{i^{*}} v_{i}, \quad \bar{v}_{i^{*}}=\left(1+r_{i^{*}}\right)^{-1} v_{i} \quad \text { if } \quad x_{i^{*}} \in S^{2} .
\end{gathered}
$$

Continuing in this way, for all $i \in \hat{I}, h \in \hat{I}$ with $\iota_{h}=i$, and $l \in M$ with $j_{l}=i$, we obtain elements (i)-(v) satisfying (I1),(I2). Let $\tilde{u}_{\hat{0}}^{\prime}=u_{\hat{0}}^{\prime}$. If $l \in M$ and $j_{l}=\hat{0}$, take $\tilde{y}_{l}^{\prime}=y_{l}$.

(3) If $S=S^{2}$, let $\left(\tilde{x}_{h}^{\prime}, \tilde{v}_{h}^{\prime}\right)=\left(\bar{x}_{h}, \bar{v}_{h}\right)$ if $\iota_{h}=\hat{0}, \tilde{b}=\tilde{b}^{\prime}$, and $\tilde{v}=\tilde{v}^{\prime}$. By the inductive construction, $\tilde{b}$ and $\tilde{v}$ satisfy the requirements of the lemma. In fact, $\tilde{b} \in \mathcal{M}_{\mathcal{T}, 0}^{(H)}$. If $S=\Sigma$, we could extend the above construction to the principal component $\Sigma$ as we did for $S=S^{2}$ if $q_{\tilde{v}^{\prime}}$ were defined using the metric $g_{b, \hat{0}}$ on $\Sigma$, which may differ slightly from $g_{\tilde{b}^{\prime}, \hat{0}}$. This problem is fixed below.

(4) If $l \in M$ and $j_{l}=\hat{0}$, we take $\tilde{y}_{l}=y_{l}$ as before. For all $h \in \hat{I}$ with $\iota_{h}=\hat{0}$, let $\tilde{x}_{h} \in \Sigma, \tilde{v}_{h} \in T_{\tilde{x}_{h}} \Sigma$, and $\Theta_{h}: B_{2\left|v_{h}\right|_{b}^{-\frac{1}{2}}}(0 ; \mathbb{C}) \longrightarrow \mathbb{C}$ be such that

$\left(\Sigma_{\hat{0}} 1\right) d_{b}\left(x_{h}, \tilde{x}_{h}\right) \leq C\left(b^{*}\right) \epsilon\left|v_{h}\right|,\left.|| \tilde{v}_{h}\right|_{\tilde{b}}-\left.\left|v_{h}\right|_{b}\left|\leq C\left(b^{*}\right) \epsilon\right| v_{h}\right|_{b}$

$\left(\Sigma_{\hat{0}} 2\right)$ for all $z \in B_{b}\left(x_{h}, 2\left|v_{h}\right|_{b}^{\frac{1}{2}}\right)$,

$$
\frac{\phi_{\tilde{b}, h} z}{\tilde{v}_{h}}=\left(1+r_{h}\right)\left\{c_{h}+\frac{\phi_{b, h} z}{v_{h}}+\Theta_{h}\left(\frac{\phi_{b, h} z}{v_{h}}\right)\right\} ;
$$

$\left(\Sigma_{\hat{0}} 3\right) \Theta_{h}$ is holomorphic, $\Theta_{h}(0)=0, \Theta_{h}^{\prime}(0)=0$, and $\left\|\Theta_{h}^{\prime \prime}\right\|_{C^{0}} \leq C\left(b^{*}\right)|v|^{2} \epsilon$. 
Note that even though we have not defined $\tilde{b}$ completely yet, $\left(\Sigma_{\hat{0}} 1\right)$ and $\left(\Sigma_{\hat{0}} 2\right)$ are still well-defined statements, since the metric $g_{\tilde{b}, \hat{0}}$ on $\Sigma$ depends only on the singular points $\left\{\tilde{x}_{h}: \iota_{h}=\hat{0}\right\}$ on $\Sigma$. Existence of such $\tilde{x}_{h}, \tilde{v}_{h}$, and $\Theta_{h}$ follows from Corollary 5.5, provided $\delta$ is sufficiently small.

If $\iota_{i}=\hat{0}$ and $j_{l}=i$, let $\left(i, \tilde{y}_{l}\right)=q_{\tilde{v}, i} q_{v, i}^{-1}\left(i, y_{l}\right)$. The map $q_{\tilde{v}, i}$ is well-defined even though $\tilde{v}$ has not been defined completely yet. By $\left(\Sigma_{\hat{0}} 2\right)$,

$$
\tilde{y}_{l}=q_{\tilde{v}, i} q_{v, i}^{-1}\left(y_{l}\right)=\frac{\phi_{\tilde{b}, \tilde{x}_{i}} \phi_{b, x_{i}}^{-1}\left(y_{l} v_{i}\right)}{\tilde{v}_{i}}=\left(1+r_{i}\right)\left\{c_{i}+y_{l}+\Theta_{i}\left(y_{l}\right)\right\} .
$$

Since $\tilde{y}_{l}^{\prime}=\left(1+r_{i}\right)\left(y_{l}+c_{i}\right),\left|\tilde{y}_{l}-\tilde{y}_{l}^{\prime}\right| \leq C\left(b^{*}\right)|v|^{2} \epsilon$ by $\left(\Sigma_{\hat{0}} 3\right)$.

Suppose $h \in \hat{I}, \iota_{h} \in \hat{I}$, and for every $i \in \hat{I}$ with $i<h$ and $j \in M$ with $j_{l}=i$, we have defined

$$
\begin{aligned}
& \tilde{x}_{i} \in \Sigma_{\mathcal{T}, \iota_{i}}, \quad \tilde{y}_{l} \in \Sigma_{\mathcal{T}, i}, \quad \tilde{v}_{i} \in \begin{cases}T_{\tilde{x}_{i}} \Sigma, & \text { if } \iota_{i}=\hat{0} \\
\mathbb{C}, & \text { if } \iota_{i} \neq \hat{0}\end{cases} \\
& \tilde{c}_{i} \in \mathbb{C}, \quad \Theta_{i}: B_{2\left|v_{i}\right|_{b}^{-\frac{1}{2}}}(0 ; \mathbb{C}) \longrightarrow \mathbb{C}
\end{aligned}
$$

such that

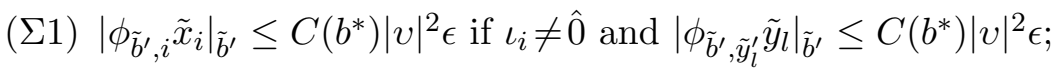

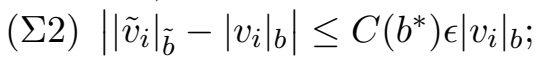

(इ3) $\left|\tilde{c}_{i}-c_{i}\right| \leq C\left(b^{*}\right)|v|^{2} \epsilon$;

$(\Sigma 4)$ for all $z \in \Sigma$ such that $r_{b, i} q_{v, \iota_{i}}(z) \leq 2\left|v_{i}\right|_{b}^{\frac{1}{2}}$,

$$
\frac{\phi_{\tilde{b}, i} q_{\tilde{v},,_{i}} z}{\tilde{v}_{i}}=\left(1+r_{i}\right)\left\{\tilde{c}_{i}+\frac{\phi_{b, i} q_{v, \iota_{i}} z}{v_{i}}+\Theta_{i}\left(\frac{\phi_{b, i} q_{v, \iota_{i}} z}{v_{i}}\right)\right\}
$$

( $\Sigma 5) \Theta_{i}$ is holomorphic, $\Theta_{i}(0)=0, \Theta_{i}^{\prime}(0)=0$, and $\left\|\Theta_{i}^{\prime \prime}\right\|_{C^{0}} \leq C\left(b^{*}\right)|v|^{2} \epsilon$.

If $h \in H$, we take $\tilde{x}_{h}=\tilde{x}_{h}^{\prime}, \tilde{v}_{h}=\tilde{v}_{h}^{\prime}=0, \tilde{y}_{l}=\tilde{y}_{l}^{\prime}$ if $j_{l}=h, \tilde{c}_{h}=c_{h}=0$, and $\Phi_{h}(z)=0$. If $h \notin H$, let

$$
\left(\iota_{h}, \tilde{x}_{h}\right)=q_{\tilde{v}, \iota_{h}} q_{v, \iota_{h}}^{-1}\left(h, \bar{x}_{h}\right) .
$$

By an argument similar to (4.18), from $(\Sigma 4)$ we obtain

$$
\tilde{x}_{h}=\left(1+r_{\iota_{h}}\right)\left\{\tilde{c}_{\iota_{h}}+\bar{x}_{h}+\Theta_{\iota_{h}}\left(\bar{x}_{h}\right)\right\} .
$$


Since $\tilde{x}_{h}^{\prime}=\left(1+r_{\iota_{h}}\right)\left(\bar{x}_{h}+c_{\iota_{h}}\right),(4.19),(\Sigma 3)$, and $(\Sigma 5)$ imply the first part of ( $\Sigma 1)$ with $i=h$. Furthermore, by assumption $(\Sigma 4)$,

$$
\begin{aligned}
& \phi_{\tilde{b}, h} q_{\tilde{v}, \iota_{h}}(z)=q_{\tilde{v}, \iota_{h}}(z)-\tilde{x}_{h} \\
& =\frac{\phi_{\tilde{b}, \iota_{h}} q_{\tilde{v}, \iota_{h}}(z)}{\tilde{v}_{\iota_{h}}}-\tilde{x}_{h} \\
& =\left(1+r_{\iota_{h}}\right)\left\{\left(\frac{\phi_{b, \iota_{h}} q_{v, \iota_{h}}(z)}{v_{\iota_{h}}}-\bar{x}_{h}\right)+\left(\Theta_{\iota_{h}}\left(\frac{\phi_{b, \iota_{h}} q_{v, \iota_{h}}(z)}{v_{\iota_{h}}}\right)-\Theta_{\iota_{h}}\left(\bar{x}_{h}\right)\right)\right\} .
\end{aligned}
$$

Since $\Theta_{\iota_{h}}$ is holomorphic, and

$$
\frac{\phi_{b, \iota_{h}} q_{v, \iota_{\iota_{h}}}(z)}{v_{\iota_{h}}}-\bar{x}_{h}=\phi_{b, h} q_{v, \iota_{h}}(z)+c_{h} v_{h},
$$

we can rewrite $(4.20)$ as

$$
\begin{aligned}
\phi_{\tilde{b}, h} q_{\tilde{v}, \iota_{h}}(z)=\left(1+r_{\iota_{h}}\right)\left(1+a_{h}\right) v_{h} & \\
& \cdot\left\{\tilde{c}_{h}+\frac{\phi_{b, h} q_{v, \iota_{h}}(z)}{v_{h}}+\Theta_{h}\left(\frac{\phi_{b, h} q_{v, \iota_{h}}(z)}{v_{h}}\right)\right\},
\end{aligned}
$$

where the complex numbers $a_{h}, \tilde{c}_{h} \in \mathbb{C}$ and the holomorphic function

$$
\Theta_{h}: B_{2\left|v_{h}\right|^{-\frac{1}{2}}}(0, \mathbb{C}) \longrightarrow \mathbb{C}
$$

are given by

$$
\begin{gathered}
a_{h}=\left.\frac{d}{d z} \Theta_{\iota_{h}}(z)\right|_{z=x_{h}}, \\
\left(1+a_{h}\right) \tilde{c}_{h}=c_{h}+\frac{\Theta_{\iota_{h}}\left(x_{h}\right)-\Theta_{\iota_{h}}\left(x_{h}-c_{h} v_{h}\right)}{v_{h}}, \\
\Theta_{h}(z)=\frac{\Theta_{\iota_{h}}\left(v_{h} z+x_{h}\right)-v_{h} z \Theta_{\iota_{h}}^{\prime}\left(x_{h}\right)-\Theta_{\iota_{h}}\left(x_{h}\right)}{\left(1+a_{h}\right) v_{h}} .
\end{gathered}
$$

By (4.23), $\Theta_{h}(0)=0$ and $\Theta_{h}^{\prime}(0)=0$. By our assumptions on $\Theta_{\iota_{h}},(4.22)$, and (4.23),

$$
\begin{gathered}
\left|a_{h}\right| \leq C\left(b^{*}\right)|v|^{2} \epsilon\left|x_{\iota_{h}}\right| \leq C^{\prime}\left(b^{*}\right)|v|^{2} \epsilon \\
\left|\tilde{c}_{h}-c_{h}\right| \leq C\left(b^{*}\right)\left(\epsilon\left|a_{h}\right|+\left|v_{h}\right|^{-1}|v|^{2} \epsilon\left|c_{h} v_{h}\right|\right) \leq C^{\prime}\left(b^{*}\right)|v|^{2} \epsilon \\
\left\|\Theta_{h}^{\prime \prime}\right\|_{C^{0}} \leq C\left(b^{*}\right)\left|v_{h}\right|^{-1}|v|^{2} \epsilon\left|v_{h}\right|^{2} \leq C^{\prime}\left(b^{*}\right)|v|^{2} \epsilon .
\end{gathered}
$$

We now take

$$
\tilde{v}_{h}=\left(1+a_{h}\right) \tilde{v}_{h}^{\prime}=\left(1+a_{h}\right)\left(1+r_{\iota_{h}}\right)\left(1+r_{h}\right)^{-1} v_{h} .
$$


It follows from (4.24)-(4.26) that the induction hypotheses $(\Sigma 2)-(\Sigma 5)$ with $i=h$ are satisfied. If $j_{l}=h$, let

$$
\left(h, \tilde{y}_{l}\right)=q_{\tilde{v}, h} q_{v, h}^{-1}\left(h, y_{l}\right) .
$$

By the same argument as in the case $\iota_{h}=\hat{0}$ above, $(\Sigma 3)-(\Sigma 5)$ of the $i=\iota_{h}$ case imply that the second part of $(\Sigma 1)$ with $i=h$ is satisfied. Continuing in this way, we obtain tuples

$$
\tilde{b}=\left(\Sigma, M, I ; \tilde{x},(j, \tilde{y}), \tilde{u}^{\prime}\right), \quad \tilde{c}=c_{\hat{I}}, \quad \tilde{v}=\left(\tilde{b}, \tilde{v}_{\hat{I}}\right),
$$

satisfying $(\Sigma 1)-(\Sigma 5)$. Since $\tilde{b}^{\prime} \in \mathcal{M}_{\mathcal{T}, 0}^{(H)}$, by $(\Sigma 1) \tilde{b} \in \mathcal{M}_{\mathcal{T}, \epsilon}^{(H)}$ if $\delta$ is sufficiently small. Finally, $(\Sigma 1)-(\Sigma 5)$ along with (I1) and (I2) show that $\tilde{b}$ and $\tilde{v}$ satisfy the two requirements of the lemma.

Corollary 4.13. If $\mathcal{T}$ is a simple bubble type, there exist $\mathbb{R}^{+}$-valued smooth functions $\delta, C$ on $\mathcal{M}_{\mathcal{T}}^{(0)}$ with the following property. Suppose $b^{*} \in \mathcal{M}_{\mathcal{T}}^{(0)}$, $\epsilon<\delta\left(b^{*}\right), b \in \mathcal{M}_{\mathcal{T}, \epsilon}^{(H)}$ is such that

$$
d\left(b^{*}, b\right) \leq \delta\left(b^{*}\right) \quad \text { and } \quad v=\left(b, v_{\hat{I}}\right) \in F_{b}^{(H)} \mathcal{T}_{\delta\left(b^{*}\right)} .
$$

Then there exist

$$
\tilde{b} \in \mathcal{M}_{\mathcal{T}, 0}^{(H)} \quad \text { and } \quad \tilde{v}=\left(\tilde{b}, \tilde{v}_{\hat{I}}\right) \in F_{\tilde{b}}^{(H)} \mathcal{T}
$$

such that

(1) $d(b, \tilde{b}) \leq C\left(b^{*}\right) \epsilon$ and $\left|\tilde{v}_{h}-v_{h}\right| \leq C\left(b^{*}\right) \epsilon\left|v_{h}\right|$ for all $h \in \hat{I}$;

(2) if $q_{v}(z) \in \Sigma_{\mathcal{T}, i}, r_{b, h}\left(q_{v} z\right) \geq 3\left|v_{h}\right|^{\frac{1}{2}}$ for all $h \in \hat{I}-H$ such that $\iota_{h}=i$ and $\left|q_{S}^{-1}\left(q_{v} z\right)\right| \geq 3\left|v_{i}\right|^{\frac{1}{2}}$ if $i \in \hat{I}-H$, then $d_{b}\left(q_{v}(z), q_{\tilde{v}}(z)\right) \leq \epsilon$.

Proof. If $S=S^{2}$, the tuples $\tilde{b}$ and $\tilde{v}$ constructed in the first half of the proof of Lemma 4.12 satisfy the requirements of the corollary. In fact, $d_{b}\left(q_{v}(z), q_{\tilde{v}}(z)\right)=0$ if $z$ is as in (2) above. If $S=\Sigma$, let

$$
\tilde{\epsilon}=\epsilon^{2} \prod_{h \in[I]-H}\left|v_{h}\right|_{b}^{2}>0 .
$$

If $C\left(b^{*}\right) \delta\left(b^{*}\right)$ is sufficiently small, by repeated applications of Lemma 4.12 , we can replace the tuples $b$ and $v$ by $b^{\prime} \in \mathcal{M}_{\mathcal{T}, \tilde{\epsilon}}^{(H)}$ and $v^{\prime}=\left(b^{\prime}, v_{\hat{I}}^{\prime}\right) \in F^{(H)} \mathcal{T}$ such that

(1) $d\left(b, b^{\prime}\right) \leq C^{\prime}\left(b^{*}\right) \epsilon$ and $\left|v_{h}^{\prime}-v_{h}\right| \leq C^{\prime}\left(b^{*}\right) \epsilon\left|v_{h}\right|_{b}$ for all $h \in \hat{I}$;

(2) if $q_{v}(z) \in \Sigma_{\mathcal{T}, i}, r_{b, h}\left(q_{v} z\right) \geq \frac{5}{2}\left|v_{h}\right|^{\frac{1}{2}}$ for all $h \in \hat{I}-H$ such that $\iota_{h}=i$ and $\left|q_{S}^{-1}\left(q_{v} z\right)\right| \geq \frac{5}{2}\left|v_{i}\right|^{\frac{1}{2}}$ if $i \in \hat{I}-H$, then $d_{b}\left(q_{v}(z), q_{\tilde{v}}(z)\right) \leq 2 \delta(b) \epsilon$.

Applying the construction of the first half of the proof of Lemma 4.12 to the tuples $b^{\prime}$ and $v^{\prime}$, we obtain tuples $\tilde{b} \in \mathcal{M}_{\mathcal{T}}^{(0)}$ and $\tilde{v}=\left(\tilde{b}, \tilde{v}_{\hat{I}}\right) \in F_{\tilde{b}}^{(H)} \mathcal{T}$ such that

$$
d\left(b^{\prime}, \tilde{b}\right) \leq C\left(b^{*}\right) \tilde{\epsilon} \quad \text { and } \quad\left|\tilde{v}_{h}-v_{h}^{\prime}\right| \leq C\left(b^{*}\right) \tilde{\epsilon}\left|v_{h}^{\prime}\right|_{b^{\prime}} \forall h \in \hat{I} .
$$


Then if $z$ is as in the requirement (2) of the corollary,

$$
d_{b^{\prime}}\left(q_{v^{\prime}}(z), q_{\tilde{v}}(z)\right) \leq C\left(b^{*}\right) \tilde{\epsilon}\left(\prod_{h \in \hat{I}-H}\left|v_{h}^{\prime}\right|\right)^{-1} \leq \epsilon^{2}
$$

if $\delta$ is sufficiently small. Thus, the tuples $\tilde{b}$ and $\tilde{v}$ satisfy both requirements of the corollary.

4.4. Gromov Convergence and the $L^{p}$-norm of the Differential. Let $b_{k}=\left(S, M, I ; x,\left(j, y_{k}\right), u_{k}\right)$ be a sequence of smooth maps converging to

$$
b^{*}=\left(S, M, I^{*} ; x^{*},\left(j^{*}, y^{*}\right), u^{*}\right) \in \mathcal{M}_{\mathcal{T}^{*}}^{(0)}
$$

with respect to the Gromov topology such that $\bar{\partial} u_{k, \hat{0}}=t_{k} \nu$ with $t_{k} \longrightarrow 0$ and $\bar{\partial} u_{k, h}=0$ if $h \in \hat{I}$. We assume that $\mathcal{T}^{*}$ is a simple bubble type. In the next subsection, it is proved that $b_{k}$ lies in the image of the gluing map $\tilde{\gamma}_{\mathcal{T}, t_{k} \nu}$ for some $k$. In this subsection, we show the differentials of $d u_{k, i}$ satisfy a certain condition which holds for all bubble maps in the image of $\tilde{\gamma}_{\mathcal{T}, t_{k} \nu}$.

By definition of convergence, for all $k$ sufficiently large, we can choose

(a) curves $\mathcal{C}_{k}=\left(S, M, I^{*} ; x_{k}^{\prime},\left(j^{*}, y^{*}\right)\right)$ with $\lim _{k \longrightarrow \infty} x_{k, h}^{\prime}=x_{h}^{*}$ for all $h \in \hat{I}^{*}$, and

(b) vectors $\left(v_{k}\right) \in F_{\mathcal{C}_{k}}^{(0)}$ with $16\left|v_{k}\right|_{g_{b}} \leq r_{\mathcal{C}_{k}} g_{b}$, such that $\lim _{k \longrightarrow \infty}\left|v_{k}\right|=0$, $\mathcal{C}\left(v_{k}\right)=\left(S, M, I ; x_{k},\left(j_{k}, y\left(v_{k}\right)\right)\right)$, and

$$
\begin{aligned}
& \lim _{k \longrightarrow \infty} \sup _{z \in \Sigma_{\mathcal{C}\left(v_{k}\right)}} d_{V}\left(u_{b^{*}}\left(q_{v_{k}}(z)\right), u_{b_{k}}(z)\right)=0, \\
& \lim _{k \longrightarrow \infty} q_{v_{k}}\left(j_{k, l}, y_{k, l}\right)=\left(j_{l}^{*}, y_{l}^{*}\right) \forall l \in M,
\end{aligned}
$$

where $v_{k}=\left(\mathcal{C}_{k},\left(v_{k}\right)_{\hat{I}^{*}}\right)$ and $g_{b}$ denotes the standard metric on $\Sigma_{\mathcal{C}_{k}}$ if $S=S^{2}$.

Let

$$
\phi_{k, h}=\left\{\begin{array}{ll}
\phi_{x_{k, h}^{\prime}}, & \text { if } x_{k, h}^{\prime} \in S^{2} ; \\
\phi_{g_{b, 0}, x_{k, h}^{\prime}}, & \text { if } x_{k, h}^{\prime} \in \Sigma ;
\end{array} \quad r_{k, h}= \begin{cases}r_{x_{k, h}^{\prime}}, & \text { if } x_{k, h}^{\prime} \in S^{2} \\
r_{g_{b, 0}, x_{k, h}^{\prime}}, & \text { if } x_{k, h}^{\prime} \in \Sigma .\end{cases}\right.
$$

Let $g_{v_{k}}$ be the metric on $\Sigma_{b_{k}}=\Sigma_{v_{k}}$ defined as in Subsection 3.3, using the metric $g_{b, \hat{0}}$ on $\Sigma$ if $S=\Sigma$.

For any element in the image $\tilde{\gamma}_{\mathcal{T}, t \nu}$ that lies near $b^{*}$, the modified $\left(L^{p}, g_{v_{k}}\right)$ norm of $d \tilde{u}_{v}$ is bounded above by a constant dependent only on $b^{*}$. Furthermore, as $v \longrightarrow 0$ and the size of the necks is reduced, the modified $\left(L^{p}, g_{v}\right)$-norm of $d \tilde{u}_{v}$ on such necks tends to zero. The modified $\left(L^{p}, g_{v}\right)$ norm is bounded above by the usual $\left(L^{2 p}, g_{v}\right)$-norm times some constant dependent only on $b^{*}$. In this subsection, we show that the $\left(L^{2 p}, g_{v_{k}}\right)$-norm of $d u_{b_{k}}$ is uniformly bounded and tends to zero on the "necks." Instead of 
using our usual cutoff function $\beta$, we will use the family of cutoff functions provided by the following lemma. The proof can be found in [MS, p166]. The statement below is somewhat sharper than in [MS], but the proof in [MS] suffices.

Lemma 4.14. For every $\epsilon>0$, there exists a smooth function $[0,1]$-valued function $\tilde{\beta}_{\epsilon}$ on $\mathbb{R}$ such that

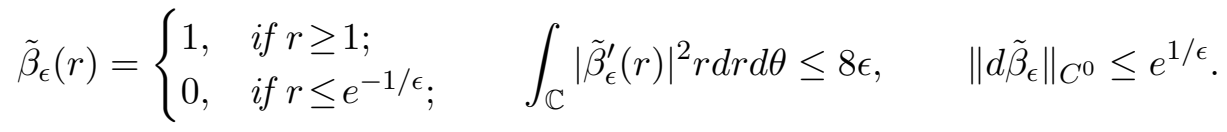

Given $r>0$, we denote by $\tilde{\beta}_{\epsilon, r}$ the cutoff function defined by $\tilde{\beta}_{\epsilon, r}(t)=$ $\tilde{\beta}_{\epsilon}\left(r^{-\frac{1}{2}} t\right)$.

We now define nearly holomorphic maps $f_{k, i} \in C^{\infty}\left(\Sigma_{\mathcal{C}_{k}, i} ; V\right)$. In order to simplify computations, we fix a finite family of $J$-invariant metrics on $V$ such that for some fixed $\varepsilon>0$ and for every $q \in V$ there exists a metric $g_{V, q}$ in this family such that $\left(B_{g_{V, q}}(q, \varepsilon), J, g_{V, q}\right)$ is isomorphic to a ball in $\mathbb{C}^{n}$. Since $V$ is compact and the family of metrics is finite, all estimates below that depend on a particular metric $g_{V, q}$ will involve bounds dependent only on $V$. We denote by $\exp _{q}$ the exponential map of (the Levi-Civita connection of) the metric $g_{V, q}$ and by $B_{q}(\epsilon)$ the $g_{V, q}$-geodesic ball about $q$ of radius $\epsilon$. If $\delta>0$ and $h \in I^{*}-I$, let

$$
\begin{aligned}
& B_{h, k}^{+}(\delta)=\left\{\left(\iota_{h}^{*}, z\right) \in \Sigma_{\mathcal{C}_{k}, \iota_{h}^{*}}: r_{k, h}\left(\iota_{h}^{*}, z\right) \leq \delta\right\}, \\
& B_{h, k}^{-}(\delta)=\left\{(h, z) \in \Sigma_{\mathcal{C}_{k}, h}:\left|q_{S}^{-1}(z)\right| \leq \delta\right\} .
\end{aligned}
$$

Choose a sequence $\epsilon_{k} \in \mathbb{R}^{+}$converging to zero. Let

$$
r_{k}=\left(2 \sum_{i \in I^{*}}\left\|d u_{i}^{*}\right\|_{b^{*}, C^{2}}\right)^{-1} \epsilon_{k} .
$$

By taking a subsequence if necessary, it can be assumed that

$$
\begin{aligned}
& \left|t_{k}\right| \leq \epsilon_{k}, \quad d_{V}\left(u_{b^{*}}\left(q_{v_{k}}(z)\right), u_{b_{k}}(z)\right) \leq \epsilon_{k} \forall z \in \Sigma_{b_{k}} \\
& r_{b^{*}, h}\left(\iota_{h}^{*}, x_{k, h}^{\prime}\right) \leq r_{k}, \quad e^{\frac{2 p}{\epsilon_{k}}}\left|v_{k, h}\right|_{b^{*}}^{\frac{1}{2}} \leq r_{k} .
\end{aligned}
$$

Let $q_{h}=u_{h}^{*}(\infty)$ and

$$
\tilde{A}_{h, k}^{ \pm}=B_{h, k}^{ \pm}\left(\left|v_{k, h}\right|_{b^{*}}^{\frac{1}{2}}\right)-B_{h, k}^{ \pm}\left(e^{-\frac{1}{\epsilon_{k}}}\left|v_{k, h}\right|_{b^{*}}^{\frac{1}{2}}\right) .
$$

By (4.28),

$$
u_{b_{k}}\left(q_{v_{k}}^{-1}\left(B_{h, k}^{ \pm}\left(e^{\frac{1}{\epsilon_{k}}}\left|v_{k, h}\right|_{b}^{\frac{1}{2}}\right)\right)\right) \subset B_{q_{h}}\left(C\left(b^{*}\right) \epsilon_{k}\right) .
$$


Thus, we can define $\xi_{k, h}^{ \pm} \in C^{\infty}\left(\tilde{A}_{h, k}^{ \pm} ; T_{q_{h}} V\right)$ by

$$
\begin{array}{lll}
\exp _{q_{h}, q_{h}} \xi_{k, h}^{+}(z)=u_{b_{k}}\left(q_{v_{k}, \iota_{h}^{*}}^{-1}\left(\iota_{h}^{*}, z\right)\right), & \left|\xi_{k, h}^{+}(z)\right|_{g_{V, q_{h}}}<\varepsilon ; \\
\exp _{q_{h}, q_{h}} \xi_{k, h}^{-}(z)=u_{b_{k}}\left(q_{v_{k}, \iota_{h}^{*}}^{-1}\left(\iota_{h}^{*}, \phi_{k, h}^{-1}\left(z v_{k, h}\right)\right)\right), & \left|\xi_{k, h}^{-}(z)\right|_{g_{V, q_{h}}}<\varepsilon,
\end{array}
$$

provided $k$ is sufficiently large (depending on $b^{*}$ ). Let $\bar{\xi}_{k, h}^{ \pm} \in T_{q_{h}} V$ be given by

$$
\bar{\xi}_{k, h}^{ \pm}=\frac{1}{\operatorname{Area}\left(\tilde{A}_{k, h}^{ \pm}\right)} \int_{\tilde{A}_{k, h}^{ \pm}} \xi_{k, h}^{ \pm},
$$

where the area and the integral are computed using the metric $g_{b^{*}, \iota_{h}^{*}}$ on $\Sigma_{b^{*}, \iota_{h}^{*}}$ and $g_{b^{*}, h}$ on $\Sigma_{b^{*}, h}$. For each $i \in I^{*}$, we define $f_{k, i} \in C^{\infty}\left(\Sigma_{b^{*}, i} ; V\right)$ as follows. If $h \in I^{*}-I$ is such that $\iota_{h}^{*}=i$ and $r_{k, h}(z) \leq\left|v_{k, h}\right|_{b^{*}}^{\frac{1}{2}}$, we put

$$
f_{k, i}(z)=\exp _{q_{h}, q_{h}}\left\{\bar{\xi}_{k, h}^{+}+\tilde{\beta}_{\epsilon_{k},\left|v_{k, h}\right|_{b^{*}}}\left(r_{k, h}(z)\right)\left(\xi_{k, h}^{+}(z)-\bar{\xi}_{k, h}^{+}\right)\right\} .
$$

If $i \in I^{*}-I$ and $\left|q_{S}^{-1}(z)\right| \leq\left|v_{k, i}\right|_{b^{*}}^{\frac{1}{2}}$, we put

$$
f_{k, i}(z)=\exp _{q_{i}, q_{i}}\left\{\bar{\xi}_{k, i}^{-}+\tilde{\beta}_{\epsilon_{k},\left|v_{k, i}\right|_{b^{*}}}\left(\left|q_{S}^{-1}(z)\right|\right)\left(\xi_{k, i}^{-}(z)-\bar{\xi}_{k, i}^{-}\right)\right\} .
$$

In all other cases, we set

$$
f_{k, i}(z)=u_{b_{k}}\left(q_{v_{k}}^{-1}(i, z)\right) \text {. }
$$

Let $\zeta_{k, i}^{\prime} \in \Gamma\left(u_{i}^{*}\right)$ be given by

$$
\exp _{b^{*}, u_{i}^{*}} \zeta_{k, i}^{\prime}=f_{k, i}, \quad\left\|\zeta_{k, i}^{\prime}\right\|_{b^{*}, C^{0}}<\operatorname{inj} g_{V, b^{*}}
$$

Lemma 4.15. There exists $C>0$ such that for all $k$ sufficiently large and $i \in I^{*}$,

$$
\left\|\zeta_{k, i}^{\prime}\right\|_{b^{*}, C^{0}} \leq C \epsilon_{k}, \quad\left\|\bar{\partial} f_{k, i}\right\|_{g_{b^{*}, i}, 2 p} \leq C \epsilon_{k}^{\frac{1}{2 p}}\left(\left\|d f_{k, i}\right\|_{g_{b^{*}, i}, 2 p}+1\right) .
$$

Proof. The first statement is clear from (4.28) and the construction of $f_{k, i}$ above. Suppose $z \in \Sigma_{b^{*}, i}$. If $z \notin B_{h, k}^{+}\left(\left|v_{k, h}\right|_{b^{*}}^{\frac{1}{2}}\right)$ for all $h \in I^{*}-I$ and $z \notin$ $B_{i, k}^{-}\left(\left|v_{k, i}\right|_{b^{*}}^{\frac{1}{2}}\right)$ if $i \in I^{*}-I$, then

$$
\left|\bar{\partial} f_{k, i}\right|_{g_{b^{*}, i}, z} \leq C_{\nu} t_{k} \leq C_{\nu} \epsilon_{k}
$$

Suppose $z \in \tilde{A}_{h, k}^{+}$with $h \in I^{*}-I$. Since the metric $g_{\mathbb{P}^{n}, q_{h}}$ is flat near $q_{h}$,

$$
\left.\bar{\partial} f_{k, i}\right|_{z}=d \exp _{q_{h}, q_{h}} \bar{\partial}\left\{\bar{\xi}_{k, h}^{+}+\tilde{\beta}_{\epsilon_{k},\left|v_{k, h}\right|_{b^{*}}}\left(r_{k, h}(\cdot)\right)\left(\xi_{k, h}^{+}-\bar{\xi}_{k, h}^{+}\right)\right\}_{z} .
$$

It follows from (4.32) that

$$
\left|\bar{\partial} f_{k, i}\right|_{g_{b^{*}, i}, z} \leq C\left(\left|v_{k, h}\right|_{b^{*}}^{-\frac{1}{2}}\left|d \tilde{\beta}_{\epsilon}\right|_{\left|v_{k, h}\right|_{b^{*}}^{-\frac{1}{2}} r_{k, h}(z)}\left|\xi_{k, h}^{+}-\bar{\xi}_{k, h}^{+}\right|_{z}+\left|\bar{\partial} \xi_{k, h}^{+}\right|_{z}\right) .
$$


By Lemma 4.14 and Poincare Lemma (see Lemma 2.6 in [Z1] applied with $r=\left|v_{h, k}\right|_{b^{*}}^{\frac{1}{2}}$ and $2 p$ instead of $p$ ), and the last assumption in (4.28),

$$
\begin{aligned}
& \|\left|v_{k, h}\right|_{b^{*}}^{-\frac{1}{2}}\left|d \tilde{\beta}_{\epsilon_{k}}\right|_{\left|v_{k, h}\right|_{b^{*}}^{-\frac{1}{2}}} r_{k, h}(\cdot) \\
& \leq\left|v_{k, h}\right|_{b^{*}}^{-\frac{p-1}{2 p}} e^{\frac{2 p-2}{2 p \cdot \epsilon}}\left\|\left|v_{k, h}^{+}\right|_{b^{*}}^{-\frac{1}{2}}\left|d \tilde{\beta}_{\epsilon_{k}}\right|_{\left|v_{k, h}\right|_{b^{*}}^{-\frac{1}{2}} r_{k, h}(\cdot)}\right\|_{g_{b^{*}, i}, L^{2}\left(\tilde{A}_{h, k}^{+}\right)}\|\|_{g_{b^{*}, i}, L^{2 p}\left(\tilde{A}_{h, k}^{+}\right)}\left\|\xi_{k, h}^{+}-\bar{\xi}_{k, h}^{+}\right\|_{b^{*}, C^{0}} \\
& \leq C\left|v_{k, h}\right|_{b^{*}}^{-\frac{p-1}{2 p}} e^{\frac{2 p-2}{2 p \cdot \epsilon}}\left|v_{k, h}\right|_{b^{*}}^{\frac{2 p-2}{2 p}}\left\|d \xi_{k, h}^{+}\right\|_{g_{b^{*}, i}, L^{2 p}\left(\tilde{A}_{h, k}^{+}\right)} \\
& \leq C^{\prime} \epsilon_{k}^{\frac{1}{2 p}}\left\|d \xi_{k, h}^{+}\right\|_{g_{b^{*}, i}, L^{2 p}\left(\tilde{A}_{h, k}^{+}\right)} \leq C^{\prime} \epsilon_{k}^{\frac{1}{2 p}}\left\|d f_{k, h}\right\|_{g_{b^{*}, i}, 2 p} .
\end{aligned}
$$

The last two equations give

$$
\left\|\bar{\partial} f_{k, i}\right\|_{g_{b^{*}, i}, L^{2 p}\left(\tilde{A}_{h, k}^{+}\right)} \leq C\left(\epsilon_{k}^{\frac{1}{2 p}}\left\|d f_{k, h}^{+}\right\|_{g_{b^{*}, i}, 2 p}+\epsilon_{k}\right) .
$$

The same estimate applies to $\left\|\bar{\partial} f_{k, i}\right\|_{g_{b^{*}, i}, L^{2 p}\left(\tilde{A}_{i, k}^{-}\right)}$if $i \in I^{*}-I$. Here the exponent of $\frac{2 p}{\epsilon_{k}}$ in (4.28) is crucial:

$$
\begin{aligned}
\|\left.\bar{\partial} \xi_{k, i}^{-}\right|_{g_{b^{*}, i}, L^{2 p}\left(\tilde{A}_{i, k}^{-}\right)} ^{2 p} & \leq \quad \int t_{k}^{2 p}\left|\nu \circ d q_{v_{k}, l_{i}^{*}}^{-1}\right|_{g_{b^{*}, l_{i}^{*}}}^{2 p}\left|v_{k, i}\right|_{b^{*}}^{2 p}\left(1+r^{2}\right)^{2 p-2} r d r d \theta \\
& \leq\left. v_{k, i}\right|_{b^{*}} ^{-\frac{1}{2}} \leq r \leq e^{\frac{1}{\epsilon_{k}}}\left|v_{i}\right|_{b^{*}}^{-\frac{1}{2}} \\
& \leq C t_{k}^{2 p}\left|v_{k, i}\right|_{b^{*}}^{2 p}\left(\left|v_{k, i}\right|^{-\frac{1}{2}} e^{\frac{1}{\epsilon_{k}}}\right)^{4 p-2} \leq C t_{k}^{2 p} .
\end{aligned}
$$

Since $f_{k, i}$ is constant on $B_{h, k}^{+}\left(e^{-\frac{1}{\epsilon_{k}}}\left|v_{k, h}\right|_{b^{*}}^{\frac{1}{2}}\right)$ for $h \in I^{*}-I$ with $\iota_{h}^{*}=i$ and on $B_{i, k}^{-}\left(e^{-\frac{1}{\epsilon_{k}}}\left|v_{k, i}\right|_{b^{*}}^{\frac{1}{2}}\right)$ if $i \in I^{*}-I$, the second claim is proved.

Corollary 4.16. There exists $C>0$ such that for all $k$ sufficiently large,

$$
\left\|d f_{k, i}\right\|_{g_{b^{*}, i}, 2 p} \leq C \quad \text { and } \quad\left\|\zeta_{k, i}^{\prime}\right\|_{g_{b^{*}, i}, 2 p, 1} \leq C \epsilon_{k}^{\frac{1}{2 p}} .
$$

Proof. By the quadratic expansion of $\bar{\partial}_{u_{i}^{*}} \zeta_{k, i}^{\prime}$ as in Subsection 3.6,

$$
D_{b^{*}, u_{i}^{*}} \zeta_{k, i}^{\prime}+N_{\bar{\partial}, u_{i}^{*}} \zeta_{k, i}^{\prime}=\bar{\partial}_{u_{i}^{*}} \zeta_{k, i}^{\prime}
$$

where

$$
\left\|\bar{\partial}_{u_{i}^{*}} \zeta_{k, i}^{\prime}\right\|_{g_{b^{*}, i}, 2 p} \leq C \epsilon_{k}^{\frac{1}{2 p}}\left(\left\|d f_{k, i}\right\|_{g_{b^{*}, i}, 2 p}+1\right)
$$

by Lemma 4.15 and

$$
\left\|N_{\bar{\partial}, u_{i}^{*}} \zeta_{k, i}^{\prime}\right\|_{g_{b^{*}, i}, 2 p} \leq C\left\|\zeta_{k, i}^{\prime}\right\|_{C^{0}}\left\|\zeta_{k, i}^{\prime}\right\|_{g_{b^{*}, i}, 2 p, 1} \leq C \epsilon_{k}\left\|\zeta_{k, i}^{\prime}\right\|_{g_{b^{*}, i}, 2 p, 1},
$$


by Proposition 2.11 in $[\mathbf{Z 1}]$ and Lemma 4.15 . Thus, by standard elliptic estimates for $u_{b^{*}}$ and (4.36)-(4.38),

$$
\begin{aligned}
\left\|\zeta_{k, i}^{\prime}\right\|_{g_{b^{*}, i}, 2 p, 1} & \leq C\left(\left\|D_{b^{*}, u_{i}} \zeta_{k, i}^{\prime}\right\|_{g_{b^{*}, i}, 2 p}+\left\|\zeta_{k, i}^{\prime}\right\|_{g_{b^{*}, i}, 2 p}\right) \\
& \leq C^{\prime} \epsilon_{k}^{\frac{1}{2 p}}\left(\left\|\zeta_{k, i}^{\prime}\right\|_{g_{b^{*}, i}, 2 p, 1}+\left\|d f_{k, i}\right\|_{g_{b^{*}, i}, 2 p}+1\right)
\end{aligned}
$$

On the other hand, since $f_{k, i}=\exp _{b^{*}, u_{i}^{*}} \zeta_{k, i}^{\prime}$,

$$
\left\|d f_{k, i}\right\|_{g_{b^{*}, i}, 2 p} \leq C\left(\left\|d u_{i}^{*}\right\|_{g_{b^{*}, i}, 2 p}+\left\|\zeta_{k, i}^{\prime}\right\|_{g_{b^{*}, i}, 2 p, 1}\right) .
$$

If $\epsilon_{k}$ is sufficiently small, the claim follows from equations (4.39) and (4.40).

Corollary 4.17. There exists $C>0$ such that for all $k$ sufficiently large, $h \in \hat{I}^{*}$, and $\delta>0$,

$$
\left\|d u_{b_{k}}\right\|_{g_{v_{k}}, L^{2 p}\left(q_{v_{k}}^{-1}\left(B_{h, k}^{ \pm}(\delta)\right)\right)} \leq C\left(\epsilon_{k}^{\frac{1}{2 p}}+\delta^{\frac{1}{p}}\right) .
$$

Proof. If $h \in \hat{I}$, the statement is immediate from Corollary 4.16; so we assume $h \in I^{*}-I$. The metric $g_{v}$ on $q_{v_{k}}^{-1}\left(B_{h, k}^{+}(\delta)\right)$ differs by a bounded factor from the metric $q_{v_{k}, \iota_{h}^{*}}^{*} g_{b^{*}, i}$. Thus,

$$
\begin{aligned}
\left\|d u_{b_{k}}\right\|_{g_{v_{k}}, L^{2 p}}\left(q_{v_{k}}^{-1}\left(B_{h, k}^{+}(\delta)\right)\right) & \leq C\left\|d\left(f_{k} \circ q_{v_{k}, \iota_{h}^{*}}^{-1}\right)\right\|_{g_{b^{*}, \iota_{h}^{*}, L^{2 p}}\left(B_{h, k}^{+}(\delta)-B_{h, k}^{+}\left(\left|v_{k, h}\right|_{b^{*}}^{\frac{1}{2}}\right)\right)} \\
& =C\left\|d f_{k, \iota_{h}^{*}}\right\|_{g_{b^{*}, \iota_{h}^{*}}, L^{2 p}}\left(B_{h, k}^{+}(\delta)-B_{h, k}^{+}\left(\left|v_{k, h}\right|_{b^{*}}^{\frac{1}{2}}\right)\right) \\
& \leq C\left\|d f_{k, \iota_{h}^{*}}\right\|_{g_{b^{*}, \iota_{h}^{*}}, L^{2 p}\left(B_{h, k}^{+}(\delta)\right)}
\end{aligned}
$$

Since $f_{k, \iota_{h}^{*}}=\exp _{b^{*}, u_{\iota}^{*}} \zeta_{k, \iota_{h}^{*}}^{\prime}$, by Corollary 4.16 ,

$$
\begin{aligned}
\left\|d f_{k, \iota_{h}^{*}}\right\|_{g_{b^{*}, \iota_{h}^{*}}, L^{2 p}\left(B_{h, k}^{+}(\delta)\right)} & \leq C\left(\left\|d u_{\iota_{h}^{*}}^{*}\right\|_{g_{b^{*}, \iota_{h}^{*}}, L^{2 p}\left(B_{h, k}^{+}(\delta)\right)}+\left\|\zeta_{k, \iota_{h}^{*}}^{\prime}\right\|_{\left.g_{b^{*}, \iota_{h}^{*}, 2 p, 1}\right)}\right) \\
& \leq C^{\prime}\left(\delta^{\frac{1}{p}}+\epsilon^{\frac{1}{2 p}}\right) .
\end{aligned}
$$

The claim for $B_{h, k}^{+}(\delta)$ follows from (4.41) and (4.42). The metric $g_{v}$ on $q_{v_{k}}^{-1}\left(B_{h, k}^{-}(\delta)\right)$ differs by a bounded factor from the metric which is the pullback of the metric $g_{b^{*}, h}$ by the map

$$
z \longrightarrow q_{N}\left(\frac{\phi_{k, h} q_{v_{k}, \iota_{h}^{*}}(z)}{v_{k, h}}\right)
$$

Thus, similarly to the above,

$$
\begin{aligned}
\left\|d u_{b_{k}}\right\|_{g_{v_{k}}, L^{2 p}\left(q_{v_{k}}^{-1}\left(B_{h, k}^{-}(\delta)\right)\right)} & \leq C\left\|d f_{k, h}\right\|_{g_{b^{*}, h}, L^{2 p}\left(B_{h, k}^{-}(\delta)\right)} \\
\left\|d f_{k, h}\right\|_{g_{b^{*}, h}, L^{2 p}\left(B_{h, k}^{-}(\delta)\right)} & \leq C\left(\delta^{\frac{1}{p}}+\epsilon^{\frac{1}{2 p}}\right) .
\end{aligned}
$$


The claim for $B_{h, k}^{-}(\delta)$ follows from (4.43) and (4.44).

4.5. Surjectivity of the Gluing Map. We continue with the notation of Subsection 4.4. In this subsection, for $k$ sufficiently large, we use Corollary 4.13 to construct

$$
\tilde{v}_{k}=\left(\tilde{b}_{k},\left(\tilde{v}_{k}\right)_{\hat{I}^{*}}\right) \in F^{(0)} \mathcal{T}_{\delta}
$$

and $\tilde{\zeta}_{k} \in \Gamma\left(u_{\tilde{v}_{k}}\right)$ such that $\tilde{b}_{k}$ is very close to $b$ in $\mathcal{M}_{\mathcal{T}}^{(0)},\left\|\tilde{\zeta}_{k}\right\|_{\tilde{v}_{k}, p, 1}$ is small, and $u_{b_{k}}=\exp _{\tilde{v}_{k}} \tilde{\zeta}_{k}$. We then look at the elements of $F^{(0)} \mathcal{T}_{\delta}$ near $\tilde{v}_{k}$ to find $\tilde{v}_{k}^{\prime}$ and $\tilde{\zeta}_{k} \in \tilde{\Gamma}_{+}\left(\tilde{v}_{k}^{\prime}\right)$ such that $u_{b_{k}}=\exp _{\tilde{v}_{k}^{\prime}} \tilde{\zeta}_{k}^{\prime}$. If $\mathcal{T}$ is semiregular, we consider only the case $\hat{I}=\emptyset$; if $\mathcal{T}$ is regular, we assume $t=0$.

Let $H=\hat{I} \subset \hat{I}^{*}$. If $\delta>0$ and $i \in I^{*}$, put

$$
\begin{gathered}
\Sigma_{i, \delta}=\left\{(i, z) \in \Sigma_{b^{*}, i}:\right. \\
r_{b^{*}, h}(i, z) \leq \delta \forall h \in \hat{I}^{*}-H \text { s.t. } \iota_{h}=i, \\
\left.\left|q_{S}^{-1}(z)\right| \geq \delta \text { if } i \in \hat{I}^{*}-H\right\} .
\end{gathered}
$$

In addition to (4.28), we can assume that our sequence satisfies

$$
\left\|\zeta_{k, i}\right\|_{g_{b^{*}, i}, C^{2}\left(\Sigma_{i, r_{k}}\right)} \leq \epsilon_{k}
$$

Let $b_{k}^{\prime}=\left(S, M, I^{*} ; x_{k}^{\prime},\left(j^{*}, y^{*}\right), u^{*}\right)$. By the second assumption in (4.28),

$$
d\left(b^{*}, b_{k}^{\prime}\right) \leq C \epsilon_{k} \Longrightarrow b_{k}^{\prime} \in \mathcal{M}_{\mathcal{T}^{*}, C \epsilon_{k}}^{(H)},
$$

since $b^{*} \in \mathcal{M}_{\mathcal{T}^{*}}^{(0)}$, where $C>0$ depends only on $b^{*}$. By the last assumption of (4.28), $\left|v_{k}\right|_{b_{k}^{\prime}} \leq C \epsilon_{k}$. Thus, if $\epsilon_{k}>0$ is sufficiently small, by Corollary 4.13, there exist

$$
\tilde{b}_{k} \in \mathcal{M}_{\mathcal{T}^{*}}^{(H)} \quad \text { and } \quad \tilde{v}_{k}=\left(\tilde{b}_{k},\left(\tilde{v}_{k}\right)_{\hat{I}^{*}}\right) \in F^{(H)} \mathcal{T}^{*}
$$

such that

(1) $d(b, \tilde{b}) \leq C^{\prime} \epsilon_{k}$ and $\left|\tilde{v}_{k, h}-v_{k, h}\right| \leq C^{\prime} \epsilon_{k}\left|v_{k, h}\right|_{b}$ for all $h \in \hat{I}^{*}$;

(2) if $q_{v_{k}}(z) \in \Sigma_{\mathcal{T}^{*}, i}, r_{b^{*}, h}\left(q_{v_{k}} z\right) \geq 3\left|v_{k, h}\right|^{\frac{1}{2}}$ for all $h \in \hat{I}^{*}-H$ such that $\iota_{h}^{*}=i$ and $\left|q_{S}^{-1} q_{v_{k}}(z)\right| \geq 3\left|v_{k, i}\right|^{\frac{1}{2}}$ if $i \in I^{*}-H$, then $d_{b}\left(q_{v_{k}}(z), q_{\tilde{v}_{k}}(z)\right) \leq \epsilon_{k}$.

It then follows from the second and third assumptions of (4.28) that there exist $\tilde{\zeta}_{k} \in \Gamma\left(u_{\tilde{v}_{k}}\right), \tilde{w}_{k, h} \in T_{x_{h}\left(\tilde{v}_{k}\right)} \Sigma_{\tilde{v}_{k}, \iota_{h}}$ for $h \in H$, and $\tilde{w}_{k, l} \in T_{y_{l}\left(\tilde{v}_{k}\right)} \Sigma_{\tilde{v}_{k}, j_{l}}$ for $l \in M$ such that

$$
\begin{gathered}
\exp _{\tilde{v}_{k}} \tilde{\zeta}_{k}=u_{b_{k}}, \quad \exp _{g_{\tilde{v}_{k}}, x_{k, h}\left(\tilde{v}_{k}\right)} \tilde{w}_{k, h}=x_{k, h}, \quad \exp _{g_{\tilde{v}_{k}}, y_{k, l}\left(\tilde{v}_{k}\right)} \tilde{w}_{k, l}=y_{k, l} \\
\left\|\tilde{\zeta}_{k}\right\|_{b^{*}, C^{0}},\left|\tilde{w}_{k, h}\right|_{g_{\tilde{v}_{k}}, x_{k, h}\left(\tilde{v}_{k}\right)}\left|\tilde{w}_{k, l}\right|_{g_{\tilde{v}_{k}}, y_{k, l}\left(\tilde{v}_{k}\right)} \leq C^{\prime} \epsilon_{k} .
\end{gathered}
$$

Lemma 4.18. There exists $C>0$ such that for all $k$,

$$
\left\|\tilde{\zeta}_{k}\right\|_{\tilde{v}_{k}, p, 1} \leq C \epsilon_{k}^{\frac{1}{2 p}} .
$$


Proof. By (4.45), (1), and (2), $\left\|\tilde{\zeta}_{k}\right\|_{g_{v}, C^{1}} \leq C \epsilon_{k}$ outside of the necks

$$
\tilde{A}_{k, h}=q_{v_{k}}^{-1}\left(B_{k, h}^{+}\left(r_{k}\right) \cup B_{k, h}^{-}\left(r_{k}\right)\right) .
$$

On the other hand, $\left\|d u_{\tilde{v}_{k}}\right\|_{\tilde{v}_{k}, C^{0}} \leq C$ by Lemma 3.5 and

$$
\left\|d u_{\tilde{v}_{k}}\right\|_{\tilde{v}_{k}, L^{p}\left(\tilde{A}_{k, h}\right)} \leq C\left(\epsilon_{k}^{\frac{1}{2 p}}+r_{k}^{\frac{1}{p}}\right) \leq C^{\prime} \epsilon_{k}^{\frac{1}{2 p}}
$$

by Corollary 4.17. The three estimates imply the claim.

Suppose $\hat{I}=\emptyset$ and thus $H=\emptyset$. If $k$ is sufficiently large and $\varpi \in T_{\tilde{v}_{k}} F^{\emptyset} \mathcal{T}^{*}$ is such that $2\|\varpi\|_{\tilde{v}_{k}}<\delta\left(b^{*}\right)$, where $\delta$ is as in Lemmas 3.6 and 4.6, let

$$
\tilde{b}_{k}(\varpi)=\tilde{b}_{t \nu}\left(\tilde{v}_{k}(\varpi)\right)=\left(S, M,\{\hat{0}\} ;,(\hat{0}, \bar{y}(\varpi)), \tilde{u}_{\varpi, t \nu}\right)
$$

be the tuple defined as in Subsections 3.4 and 3.6. Let $\tilde{\zeta}_{k}(\varpi) \in \Gamma\left(\tilde{u}_{\varpi, t \nu}\right)$ and $\tilde{w}_{k, l}(\varpi) \in T_{y_{l}(\varpi)} \Sigma_{\varpi, j_{l}}$ for $l \in M$ be given by

$$
\begin{gathered}
\exp _{\tilde{v}_{k}(\varpi)} \tilde{\zeta}_{k}(\varpi)=u_{b_{k}}, \quad \text { and } \quad \exp _{\tilde{v}_{\tilde{v}_{k}(\varpi)}, y_{k, l}(\varpi)} \tilde{w}_{k, l}(\varpi)=y_{k, l}, \\
\text { and } \quad\left\|\tilde{\zeta}_{k}(\varpi)\right\|_{b^{*}, C^{0}},\left|\tilde{w}_{k, l}(\varpi)\right|_{\tilde{v}_{k}, y_{k, l}(\varpi)} \leq 2 C^{\prime} \epsilon_{k} .
\end{gathered}
$$

We need to find $\varpi$ such that $\tilde{\pi}_{\varpi,-} \tilde{\zeta}_{k}(\varpi)=0$ and $y_{l}(\varpi)=y_{k, l}$, or equivalently

$$
S_{\varpi} \tilde{\pi}_{\varpi,-} \tilde{\zeta}_{k}(\varpi)=0 \quad \text { and } \quad S_{\varpi} \tilde{w}_{k, l}(\varpi)=0,
$$

where $S_{\varpi} \tilde{w}_{k, l}(\varpi)$ denotes the parallel transport of $\tilde{w}_{k, l}(\varpi)$ back to $y_{l}\left(\tilde{v}_{k}\right)$ along the $g_{v}$-geodesic

$$
s \longrightarrow \exp _{y_{l}\left(\tilde{v}_{k}\right)} s w_{l}(\varpi) .
$$

Lemma 4.19. There exists $C>0$ such that for all $k$ sufficiently large and $\varpi, \varpi^{\prime} \in T_{\tilde{v}_{k}} F^{(\emptyset)} \mathcal{T}^{*}$ with $2\|\varpi\|_{\tilde{v}_{k}}<\delta\left(b^{*}\right)$,

$$
\begin{aligned}
& S_{\varpi} \tilde{\pi}_{\varpi,-} \tilde{\zeta}_{k}(\varpi)=\tilde{\pi}_{v,-} \tilde{\zeta}_{k}+\tilde{N}^{(0)}\left(\tilde{\zeta}_{k}, \varpi\right)-\tilde{\pi}_{v,-} \zeta_{\varpi}+N^{(0)}(\varpi), \\
& S_{\varpi} \tilde{w}_{k, l}(\varpi)=\tilde{w}_{k, l}+\tilde{N}^{(l)}\left(\tilde{w}_{k, l}, \varpi\right)-w_{l}(\varpi)+N^{(l)}(\varpi) \quad \forall l \in M,
\end{aligned}
$$

where $\zeta_{\varpi}$ is as in Subsection 3.4 and $\tilde{N}^{(l)}$ and $N^{(l)}$ satisfy

$$
\begin{array}{r}
\left|\tilde{N}^{(l)}\left(\varpi, \tilde{w}_{k, l}\right)-\tilde{N}^{(l)}\left(\varpi^{\prime}, \tilde{w}_{k, l}\right)\right|_{g_{\tilde{v}_{k}}, y_{l}\left(\tilde{v}_{k}\right)} \leq C\left\|\tilde{w}_{k, l}\right\|_{g_{v_{k}}, y_{l}\left(\tilde{v}_{k}\right)}\left\|\varpi-\varpi^{\prime}\right\|_{\tilde{v}_{k}} ; \\
\left\|N^{(0)}(\varpi)-N^{(0)}\left(\varpi^{\prime}\right)\right\|_{\tilde{v}_{k}, 2} \leq C\left(\|\varpi\|_{\tilde{v}_{k}}+\left\|\varpi^{\prime}\right\|_{\tilde{v}_{k}}\right)\left\|\varpi-\varpi^{\prime}\right\|_{\tilde{v}_{k}} ; \\
\left|N^{(l)}(\varpi)-N^{(l)}\left(\varpi^{\prime}\right)\right|_{g_{\tilde{v}_{k}}, y_{l}\left(\tilde{v}_{k}\right)} \leq C\left(\|\varpi\|_{\tilde{v}_{k}}+\left\|\varpi^{\prime}\right\|_{\tilde{v}_{k}}\right)\left\|\varpi-\varpi^{\prime}\right\|_{\tilde{v}_{k}} .
\end{array}
$$

for all $l \in M$. 
Proof. This lemma follows from a pointwise Riemannian geometry estimate on $S_{\varpi} \tilde{\zeta}_{k}(\varpi)-\left(\tilde{\zeta}_{k}-\tilde{\zeta}_{\varpi}\right)$ and the fact that all statements in Lemmas 3.6 and 4.6 can be written in a form similar to (4.47), e.g., for all $\xi \in \Gamma\left(\tilde{v}_{k}\right)$

$$
\left\|S_{\varpi} \tilde{\pi}_{\varpi,-} R_{\varpi} \xi-S_{\varpi^{\prime}} \tilde{\pi}_{\varpi^{\prime},-} R_{\varpi^{\prime}} \xi\right\|_{\tilde{v}_{k}, 2} \leq C\left\|\varpi-\varpi^{\prime}\right\|_{\tilde{v}_{k}, 2}\|\xi\|_{\tilde{v}_{k}, 2} .
$$

The latter fact can be seen from the two lemmas and the definitions of $R_{\varpi}$ and $S_{\varpi}$ in Subsection 3.4.

Lemma 4.20. There exist $C, \delta \in C^{\infty}\left(\mathcal{M}_{\mathcal{T}^{*}}^{(0)}, \mathbb{R}^{+}\right)$such that for all $v \in F^{(H)} \mathcal{T}_{\delta}^{*}$ and $\varpi \in T_{v} F^{(H)} \mathcal{T}_{\delta}^{*}$ with $\|\varpi\|_{\tilde{v}_{k}} \leq \delta(b)$,

$$
\left\|\zeta_{\varpi}\right\|_{v, 2} \leq C(b)\left\|\tilde{\pi}_{v,-} \zeta_{\varpi}\right\|_{v, 2}
$$

Proof. It can be seen directly from the definitions that

$$
\left\|\zeta_{\varpi}\right\|_{v, 2} \leq\left(1+C\left(b_{v}\right)|v|\right)\left\|\pi_{v,-} \zeta_{\varpi}\right\|_{v, 2} .
$$

The claim then follows from the proof of $(2 \mathrm{~b})$ of Lemma 3.12.

Corollary 4.21. There exist a neighborhood $U$ of $b^{*}$ in $\mathcal{M}_{\mathcal{T}^{*}}^{(0)}$ and $\delta, \epsilon>0$ such that for all $\left.v \in F^{(\emptyset)} \mathcal{T}_{\delta}^{*}\right|_{U}, \xi \in \tilde{\Gamma}_{-}(v)$ with $\|\xi\|_{v, 2}<\delta$, and $w_{l} \in T_{y_{l}(v)} \Sigma_{v, j_{l}}$ for $l \in M$ with $\left|w_{l}\right|_{g_{v}, y_{l}(v)}<\delta$, the system of equations

$$
\tilde{\pi}_{v,-} \zeta_{\varpi}-N^{(0)}(\varpi)=\xi, \quad w_{l}(\varpi)-N^{(l)}(\varpi)=w_{l} \forall l \in M,
$$

has a (unique) solution $\varpi \in T_{v} F^{(\emptyset)} \mathcal{T}$ with $\|\varpi\|_{v}<\epsilon$.

Proof. By Lemmas 3.6 and 4.20,

$$
C^{-1}\|\varpi\|_{v} \leq\left\|\tilde{\pi}_{v,-} \xi\right\|_{v, 2}+\sum_{l \in M}\left|w_{l}(\varpi)\right|_{g_{v}, y_{l}(v)} \leq C\|\varpi\|_{v}
$$

whenever $b_{v}$ lies near $b^{*}$. Thus, the claim follows from (4.47) by the usual contraction-principle argument.

Corollary 4.22. Let $\mathcal{T}^{*}=\left(S, M, I^{*} ; j^{*}, \underline{\lambda}^{*}\right)$ be a simple bubble type. If $\mathcal{T}^{*}$ is regular, the map

$$
\tilde{\gamma}_{\mathcal{T}^{*}}: F \mathcal{T}_{\delta}^{*} \longrightarrow \overline{\mathcal{M}}_{\left\langle\mathcal{T}^{*}\right\rangle}
$$

contains a neighborhood of $\mathcal{M}_{\mathcal{T} *}$ in $\overline{\mathcal{M}}_{\left\langle\mathcal{T}^{*}\right\rangle}$. If $\mathcal{T}^{*}$ is semiregular, $H=\emptyset$, and $k$ is sufficiently large, there exists $\tilde{v}_{k} \in F^{(\emptyset)} \mathcal{T}_{\delta}^{*}$ such that $b_{k}=\tilde{\gamma}_{\mathcal{T}^{*}, t_{k} \nu}\left(\tilde{v}_{k}\right)$.

Proof. The second statement is immediate from Lemmas 4.18 and 4.19 and Corollary 4.21. If $\mathcal{T}^{*}$ is regular, what we have shown is that the image of $\tilde{\gamma}_{\mathcal{T}^{*}}$ contains a neighborhood of $\mathcal{M}_{\mathcal{T}^{*}}$ in $\mathcal{M}_{\left\langle\mathcal{T}^{*}\right\rangle} \cup \mathcal{M}_{\mathcal{T}^{*}}$. Furthermore, there exists a sequence of neighborhoods $U_{1} \supset U_{2} \supset$. . of $b^{*}$ in $\overline{\mathcal{M}}_{\left\langle\mathcal{T}^{*}\right\rangle}$ such that $\bigcap U_{k}=\left\{\left[b^{*}\right]\right\}$. If $\left[b_{k}\right] \in \mathcal{M}_{\mathcal{T}}$ is a sequence of bubble maps converging to $\left[b^{*}\right] \in \mathcal{M}_{\mathcal{T}^{*}}$, it can be assumed that $\left[b_{k}\right] \in U_{k}$. By the above statement applied to $\mathcal{T}$, we can choose sequences

$$
\left\{\left[b_{k r}\right]\right\} \subset \mathcal{M}_{\left\langle\mathcal{T}^{*}\right\rangle}=\mathcal{M}_{\langle\mathcal{T}\rangle}
$$


such that for each fixed $k$ the sequence $\left\{\left[b_{k r}\right]\right\}$ converges to $\left[b_{k}\right]$. Since $U_{k}$ is an open neighborhood of $\left[b_{k}\right]$, it can be assumed that $\left[b_{k r}\right] \in U_{k}$ for all $r$. By the above, the image of $\left.\tilde{\gamma}_{\mathcal{T}^{*}}\right|_{F \mathcal{T}_{\delta / 2}^{*}}$ contains $U_{k} \cap \mathcal{M}_{\left\langle\mathcal{T}^{*}\right\rangle}$ if $k$ is sufficiently large. Thus, for all $r$ there exists $v_{k r} \in F \mathcal{T}_{\delta / 2}^{*}$ such that $\tilde{\gamma}_{\mathcal{T}^{*}}\left(v_{k r}\right)=\left[b_{k r}\right]$. Let $\tilde{v}_{k} \in F \mathcal{T}_{\delta}^{*}$ be the limit of the sequence $v_{k r}$ with $k$ fixed. Then, by continuity of the map $\tilde{\gamma}_{\mathcal{T}^{*}}$, see Corollary 4.5 ,

$$
\tilde{\gamma}_{\mathcal{T}^{*}}\left(\tilde{v}_{k}\right)=\lim _{r \longrightarrow \infty} \tilde{\gamma}_{\mathcal{T}^{*}}\left(\tilde{v}_{k r}\right)=\lim _{r \longrightarrow \infty}\left[b_{k r}\right]=\left[b_{k}\right] .
$$

Thus, the image of $\tilde{\gamma}_{\mathcal{T}^{*}}$ contains a neighborhood of $\mathcal{M}_{\mathcal{T}^{*}}$ in $\overline{\mathcal{M}}_{\left\langle\mathcal{T}^{*}\right\rangle}$.

Corollary 4.23. If $\mathcal{T}^{*}=\left(S, M, I^{*} ; j^{*}, \underline{\lambda}^{*}\right)$ is a simple regular bubble type, the map

$$
\tilde{\gamma}_{\mathcal{T}^{*}}: F \mathcal{T}_{\delta}^{*} \longrightarrow \overline{\mathcal{M}}_{\left\langle\mathcal{T}^{*}\right\rangle}
$$

is a homeomorphism onto an open neighborhood of $\mathcal{M}_{\mathcal{T}^{*}}$ in $\overline{\mathcal{M}}_{\left\langle\mathcal{T}^{*}\right\rangle}$ provided $\delta \in C^{\infty}\left(\mathcal{M}_{\mathcal{T}^{*}} ; \mathbb{R}^{+}\right)$is sufficiently small.

Proof. By Corollaries 4.5, 4.10, 4.22, the map $\tilde{\gamma}_{\mathcal{T}^{*}}: F \mathcal{T}_{\delta}^{*} \longrightarrow \overline{\mathcal{M}}_{\left\langle\mathcal{T}^{*}\right\rangle}$ is a continuous bijection onto a neighborhood of $\mathcal{M}_{\mathcal{T}^{*}}$ in $\overline{\mathcal{M}}_{\left\langle\mathcal{T}^{*}\right\rangle}$. In addition, the proof of Corollary 4.22 shows that $\tilde{\gamma}_{\mathcal{T}^{*}}$ is an open map.

\section{Appendix}

5.1. Properties of Smooth Families of Metrics on $\Sigma$. Let $m$ be a positive integer and

$$
\aleph=\left\{x=x_{[m]}: x_{h} \in \Sigma, x_{h} \neq x_{l} \text { if } h \neq l\right\} .
$$

Suppose $\left\{g_{x}: x \in \aleph\right\}$ is a smooth family of metrics on $\Sigma$ such that for any $x=x_{[m]} \in \aleph$ the metric $g_{x}$ is flat on a neighborhood of $x_{h}$ in $\Sigma$ for all $h \in[m]$. If $x=x_{[m]} \in \aleph$ and $v \in T_{y} \Sigma$, let

$$
T_{x} \aleph=\bigoplus_{h \in[m]} T_{x_{h}} \Sigma,|v|_{x}=|v|_{g_{x}, y} .
$$

If $w=w_{[m]} \in T_{x} \aleph$, let $|w|$ denote $\sum_{h \in[m]}\left|w_{h}\right|_{x}$. Define $x(w) \in \Sigma^{m}$ by

$$
x(w)=\left(x_{1}(w), \ldots, x_{m}(w)\right)=\left(\exp _{g_{x}, x_{1}} w_{1}, \ldots, \exp _{g_{x}, x_{m}} w_{m}\right) .
$$

We denote by $\phi_{x, y}$ the map $\phi_{g_{x}, y}$ and by $B_{x}(y, \delta)$ the set $B_{g_{x}}(y, \delta)$ described in Subsection 1.3. If $\delta: \aleph \longrightarrow \mathbb{R}$, let

$$
T \aleph_{\delta}=\left\{(x, w): x \in \aleph ; w \in T_{x} \aleph,|w|_{x}<\delta(x)\right\} .
$$

Lemma 5.1. There exist $\delta \in C^{\infty}\left(\aleph ; \mathbb{R}^{+}\right)$and a smooth families of holomorphic maps

$$
\left\{\tilde{p}_{h,(x, w)}:\left\{z \in B_{x}\left(x_{h}, \delta(x)\right)\right\} \longrightarrow \Sigma \mid(x, w) \in T \aleph_{\delta}\right\},
$$


such that each map $\tilde{p}_{h,(x, w)}$ is a $\left(g_{x}, g_{x(w)}\right)$-isometry,

$$
\begin{gathered}
\left.d \phi_{x, x_{h}}\right|_{x_{h}(w)} \phi_{x(w), x_{h}(w)} \tilde{p}_{h,(x, w)}(z)=\phi_{x, x_{h}}(z), \\
\text { and } \quad d_{g_{x}}\left(z, \tilde{p}_{h,(x, w)}(z)\right) \leq 2|w|_{x} \quad \forall z \in B_{x}\left(x_{h}, \delta(x)\right) .
\end{gathered}
$$

In particular, both sides of (5.1) are defined.

Proof. We choose $\delta$ such that if $w \in T_{x} \aleph$ and $|w| \leq 4 \delta(x)$, then $x(w) \in \aleph$ and the metric $g_{x(w)}$ is flat on $B_{x}\left(x_{h}, 2 \delta(x)\right)$. This choice of $\delta$ insures that both sides of (5.1) are defined. Equation (5.1) is equivalent to

$$
\begin{aligned}
\phi_{x(w), x_{h}(w)} \tilde{p}_{h,(x, w)}(z) & =\left.d \exp _{g_{x}, x_{h}}\right|_{w_{h}} \phi_{x, x_{h}}(z) \\
& =\phi_{x, x_{h}(w)} z+\left.d \exp _{g_{x}, x_{h}}\right|_{w_{h}} w_{h},
\end{aligned}
$$

since the metric $g_{x}$ is flat on $B_{x}\left(x_{h}, 2 \delta(x)\right)$. This equation defines the required map $\tilde{p}_{h,(x, w)}$. Since the metrics $g_{x}$ and $g_{x(w)}$ are flat on $B_{x}\left(x_{h}, 2 \delta(x)\right)$, the maps $\phi_{x, x_{h}(w)} z$ and $\phi_{x(w), x_{h}(w)}$ are holomorphic, and thus $\tilde{p}_{h,(x, w)}$ is holomorphic. Taking the differential of (5.2), we obtain

$$
\left.\left.d \phi_{x(w), x_{h}(w)}\right|_{\tilde{p}_{h,(x, w)}(z)} \circ d \tilde{p}_{h,(x, w)}\right|_{z}=\left.d \phi_{x, x_{h}(w)}\right|_{z} .
$$

Since $\phi_{x(w), x_{h}(w)}$ and $\phi_{x, x_{h}(w)}$ are $\left(g_{x(w)}, g_{x(w)}\right)$ - and $\left(g_{x}, g_{x}\right)$-isometries, respectively, on $B_{x}\left(x_{h}, 2 \delta(x)\right)$, it follows that $\tilde{p}_{h,(x, w)}$ is a $\left(g_{x}, g_{x(w)}\right)$-isometry on $B_{x}\left(x_{h}, 2 \delta(x)\right)$. By $(5.2)$,

$$
\begin{aligned}
d_{g_{x}}\left(z, \tilde{p}_{h,(x, w)}(z)\right) & \leq\left|w_{h}\right|_{x}+\left|\left(\phi_{x(w), x_{h}(w)}-\phi_{x, x_{h}(w)}\right) \tilde{p}_{h,(x, w)}(z)\right|_{x} \\
& \leq\left|w_{h}\right|_{x}+C(x)|w| \delta(x),
\end{aligned}
$$

since the family of metrics is smooth. If $C(x) \delta(x)<1$, the remaining claim of the lemma follows from (5.4).

Lemma 5.2. There exist $\delta, C_{k} \in C^{\infty}\left(\aleph ; \mathbb{R}^{+}\right)$, where $k$ is a positive integer, $\alpha_{h} \in C^{\infty}\left(T \aleph_{\delta} ; \mathbb{C}\right)$, and smooth families of maps

$$
\left\{\Theta_{w, h}:\left\{v \in T_{x_{h}} \Sigma:|v|_{x}<\delta(x)\right\} \longrightarrow T_{x_{h}} \Sigma \mid(x, w) \in T \aleph_{\delta}\right\}
$$

such that every map $\Theta_{w, h}$ is holomorphic,

$$
\Theta_{w, h}(0)=0, \quad \Theta_{w, h}^{\prime}(0)=0, \quad\left\|\Theta_{w, h}^{\langle k\rangle}\right\|_{C^{0}} \leq C_{k}(x)|w|, \quad\left|\alpha_{h}(w)\right| \leq C_{0}(x)|w|
$$

and

$$
\begin{array}{r}
\left.\left.d \phi_{x, x_{h}}\right|_{x_{h}(w)} d \phi_{x(w), x_{h}(w)}\right|_{x_{h}}\left(\phi_{x(w), x_{h}} z\right) \\
=\left(1+\alpha_{h}(w)\right) \phi_{x, x_{h}} z+\Theta_{w, h}\left(\phi_{x, x_{h}} z\right) .
\end{array}
$$

for all $z \in B_{x}\left(x_{h}, \delta(x)\right)$ In particular, both sides of (5.5) are defined. 
Proof. We choose $\delta$ such that if $w \in T_{x} \aleph$ and $|w| \leq 4 \delta(x)$, then $x(w) \in \aleph$ and the metric $g_{x(w)}$ is flat on $B_{x}\left(x_{h}, 4 \delta(x)\right)$. This choice of $\delta$ insures that both side of (5.5) are defined. If $w$ and $z$ are as in the statement of the lemma, by the flatness of the metric $g_{x(w)}$ near $x_{h}$, C-linearity of the differential of the exponential map near zero, and the smoothness of the family of the metrics

$$
\left.\left.d \phi_{x, x_{h}}\right|_{x_{h}(w)} d \phi_{x(w), x_{h}(w)}\right|_{x_{h}}\left(\phi_{x(w), x_{h}} z\right)=\left(1+a_{h}(w)\right)\left(\phi_{x(w), x_{h}} z\right),
$$

for some $a_{h} \in C^{\infty}\left(T \aleph_{\delta} ; \mathbb{C}\right)$ such that $a_{h}(0)=0$. Note that if $g_{x(w)}=g_{x}$, $a_{h}(w)=0$, since the metric $g_{x}$ is flat on $B_{x}\left(x_{h},|w|\right)$. The map

$$
\left\{v \in T_{x_{h}} \Sigma:|v|_{x}<2 \delta(x)\right\} \longrightarrow T_{x_{h}} \Sigma, \quad v \longrightarrow \phi_{x(w), x_{h}} \phi_{x, x_{h}}^{-1} v-v,
$$

is holomorphic since $\phi_{x(w), x_{h}}$ and $\phi_{x, x_{h}}$ are, and vanishes at 0 . Thus,

$$
\phi_{x(w), x_{h}} \phi_{x, x_{h}}^{-1} v=\left(1+b_{h}(w)\right) v+\Theta_{w, h}(v),
$$

for some $b_{h}(w) \in \mathbb{C}$ and holomorphic function $\Theta_{w, h}$ such that

$$
\Theta_{w, h}(0), \Theta_{w, h}^{\prime}(0)=0 .
$$

Equation (5.5) follows from (5.6) and (5.7). Smoothness of $b_{h}$ and $\Theta_{\cdot, h}$ in $w$ follows from the smoothness of the family of the metrics. The bounds on $\alpha_{h}$ and the derivatives of $\Theta_{w, h}$ follow from their smoothness and compactness of the fibers of

$$
\left\{w \in T_{x} \aleph:|w| \leq \delta(x)\right\} \longrightarrow \aleph .
$$

Lemma 5.3. There exist $\delta, C \in C^{\infty}\left(\aleph ; \mathbb{R}^{+}\right)$and smooth families of maps

$$
N_{h}:\left\{(x, w): x \in \aleph ;(x, w) \in T \aleph_{\delta}\right\} \longrightarrow T \aleph
$$

such that $\left|N_{h}\left(w, w_{h}\right)\right|_{x} \leq C(x)|w|\left|w_{h}\right|$ and

$$
\left.d \phi_{x, x_{h}}\right|_{x_{h}(w)}\left(\phi_{x(w), x_{h}(w)} x_{h}\right)=-w_{h}+N_{h}\left(w, w_{h}\right) .
$$

In particular, the left-hand side of (5.8) is defined.

Proof. We take $\delta$ as in Lemma 5.2. Then, the left-hand side of (5.8) is defined and

$$
\begin{aligned}
\left.d \phi_{x, x_{h}}\right|_{x_{h}(w)}\left(\phi_{x(w), x_{h}(w)} x_{h}\right) & =\left.d \phi_{x, x_{h}}\right|_{x_{h}(w)}\left(\phi_{x, x_{h}(w)} x_{h}\right) \\
& +\left.d \phi_{x, x_{h}}\right|_{x_{h}(w)}\left\{\left(\phi_{x(w), x_{h}(w)} x_{h}\right)-\left(\phi_{x, x_{h}(w)} x_{h}\right)\right\} \\
& =-w_{h}+N_{h}\left(w, w_{h}\right),
\end{aligned}
$$

where $N(\cdot, \cdot)$ is some smooth function of both variables, that vanishes if either input is zero. Equation (5.8) is thus proved, while the bound on $N_{h}$ is obtained from its smoothness and compactness of the fibers as in the proof of Lemma 5.2. 
Lemma 5.4. There exists $\delta \in C^{\infty}\left(\aleph ; \mathbb{R}^{+}\right)$such that for all

$x \in \aleph, v \in T_{x} \aleph$ s.t. $|v|<\delta(x) \quad$ and $\quad c=c_{[m]} \in \mathbb{C}^{m}$ s..t $|c||v|<\delta(x)$, there exists $w \in T \aleph$ with $\left|w_{h}\right|_{x}<2\left|c_{h}\right|\left|v_{h}\right|_{x}$ such that for all $z \in$ $B_{x}\left(x_{h}, 4 \delta(x)^{\frac{1}{2}}\right)$,

$$
\left.d \phi_{x, x_{h}}\right|_{x_{h}(w)}\left(\phi_{x(w), x_{h}(w)} z\right)=\left(1+\alpha_{h}(w)\right)\left(c_{h} v_{h}+\phi_{x, x_{h}} z\right)+\Theta_{w, h}\left(\phi_{x, x_{h}} z\right)
$$

where $\alpha_{h}(w)$ and $\Theta_{w, h}$ are as in Lemma 5.2. In particular, both sides of (5.10) are defined.

Proof. We start by choosing $\delta$ so that $8 \delta^{\frac{1}{2}}$ is smaller that the function $\delta$ of Lemmas 5.2 and 5.3. By flatness of the metric $g_{x(w)}$ on $B\left(x_{h}, 8 \delta(x)^{\frac{1}{2}}\right)$ for $w \in T_{x} \aleph$ with $|w|<\delta(x)$

$$
\phi_{x(w), x_{h}(w)} z=\left.d \phi_{x(w), x_{h}(w)}\right|_{x_{h}} \phi_{x(w), x_{h}} z+\phi_{x(w), x_{h}(w)} x_{h}
$$

for any $z \in B\left(x_{h}, 4 \delta(x)^{\frac{1}{2}}\right)$. Taking $\left.d \phi_{x, x_{h}}\right|_{x_{h}(w)}$ of both sides of (5.11) and applying Lemmas 5.2 and 5.3, we obtain

$$
\begin{array}{r}
\left.d \phi_{x, x_{h}}\right|_{x_{h}(w)}\left(\phi_{x(w), x_{h}(w)} z\right)=\left(1+\alpha_{h}(w)\right) \phi_{x, x_{h}} z+\Theta_{w, h}\left(\phi_{x, x_{h}} z\right) \\
-w_{h}+N_{h}\left(w, w_{h}\right) .
\end{array}
$$

Thus, we need to solve the equations

$$
-w_{h}+N_{h}\left(w, w_{h}\right)=\left(1+\alpha_{h}(w)\right) c_{h} v_{h} .
$$

Let $\Psi_{h}(w)=N_{h}\left(w, w_{h}\right)-\left(1+\alpha_{h}(w)\right) c_{h} v_{h}$. If $|w| \leq 2\left|c_{h}\right|\left|v_{h}\right|$, then by Lemmas 5.2 and 5.3,

$$
\begin{aligned}
& |\Psi(w)| \leq C(x)|c||v|(2|c||v|+1) \leq 2|c||v| \\
& \left|\Psi(w)-\Psi\left(w^{\prime}\right)\right| \leq C(x)|c||v|\left|w-w^{\prime}\right| \leq \frac{1}{2}\left|w-w^{\prime}\right|,
\end{aligned}
$$

provided $4 C(x) \delta(x)<1$. In such a case, $\Psi$ is a contracting operator, and thus (5.13) has a unique solution $w \in T_{x} \aleph$ with $|w|<2|c||v|$. The estimate $\left|w_{h}\right|<2\left|c_{h} \| v_{h}\right|$ follows directly from (5.13) if $\delta(x)$ is sufficiently small.

Corollary 5.5. There exist $\delta, C_{k} \in C^{\infty}\left(\aleph ; \mathbb{R}^{+}\right)$, where $k$ is a positive integer, such that for any $x \in \aleph, v \in T_{x} \aleph$ with $|v|<\delta(x), c=c_{[m]} \in \mathbb{C}^{m}$ with $|c|<\delta(x)$, and $r=r_{[m]} \in \mathbb{R}^{m}$ with $|r|<\frac{1}{2}$, there exists $\tilde{x} \in \aleph$ and $\tilde{v} \in T_{\tilde{x}} \aleph$ such that

(1) $\tilde{x}_{h} \in B_{x}\left(x_{h}, 2\left|c_{h}\right|\left|v_{h}\right|\right),\left|\frac{g_{\tilde{x}}}{g_{x}}-1\right| \leq C_{1}(x)|c||v|$,

$$
\left.|| \tilde{v}_{h}\right|_{\tilde{x}}-\left|v_{h}\right|_{x}\left|\leq C_{1}(x)\left(|c||v|+\left|r_{h}\right|\right)\right| v_{h} \mid
$$

(2) for any $z \in B_{x}\left(x_{h}, 4 \delta(x)^{1 / 2}\right)$,

$$
\frac{\phi_{\tilde{x}, \tilde{x}_{h}} z}{\tilde{v}_{h}}=\left(1+r_{h}\right)\left\{c_{h}+\frac{\phi_{x, x_{h}} z}{v_{h}}+\Theta_{v, c, r, h}\left(\frac{\phi_{x, x_{h}} z}{v_{h}}\right)\right\},
$$


where $\Theta_{v, c, r, h}$ is a holomorphic function, varying smoothly with the parameters, such that

$$
\Theta_{v, c, r, h}(0)=0, \quad \Theta_{v, c, r, h}^{\prime}(0)=0, \quad\left\|\Theta_{v, c, r, h}^{\langle k\rangle}\right\|_{C^{0}} \leq C_{k}(x)|c|\left|v \| v_{h}\right|^{k-1} .
$$

Proof. Let $\delta$ be as in Lemma 5.4. Given $v$ and $c$ as in the statement of the lemma, let $w \in T_{x} \aleph$ be the element provided by Lemma 5.4. Take

$$
\tilde{x}_{h}=x_{h}(w), \quad \tilde{v}_{h}=\left(1+r_{h}\right)^{-1}\left(1+a_{h}(w)\right) d \phi_{x, x_{h}}^{-1} \mid w_{h} v_{h} .
$$

The estimates in (1) are immediate from Lemma 5.4, provided $\delta$ is sufficiently small. The inequalities in (2) arise from the smooth dependence of $w$ on $x, v$, and $c$ in Lemma 5.4, and the fact that $w$ is zero if either $v=0$ or $c=0$.

5.2. Sobolev Inequalities for the Metrics $g_{v}$. In this subsection, we prove (3) of Lemma 3.5. The reason this estimate holds is that $\left(\Sigma_{v}, g_{v}\right)$ can be written as a union of the surfaces $\left(\Sigma_{b_{v}, i}, g_{v}\right)$ with small disks missing and annuli $\left(\tilde{A}_{v, h}^{ \pm}, g_{v}\right)$ that are uniformly equivalent to annuli in $\mathbb{R}^{2}$ with the smaller radius less than half of the larger.

Suppose $\mathcal{T}=(S, M, I ; j, \underline{\lambda})$ is a bubble type and

$$
v=\left(b, v_{\hat{I}}\right)=\left((S, M, I ; x,(j, y), u), v_{\hat{I}}\right) \in F^{(0)} \mathcal{T}_{\delta} .
$$

For any $h \in \hat{I}$ and $i \in I$, put

$$
\begin{gathered}
\tilde{A}_{v, h}^{-}=q_{v, i_{h}}^{-1}\left(\left\{\left(\iota_{h}, z\right) \in \Sigma_{b_{v}, \iota_{h}}:\left(2 \delta_{\mathcal{T}}\left(b_{v}\right)\right)^{-1}\left|v_{h}\right|_{b} \leq r_{b, h}(z) \leq\left|v_{h}\right|_{b}^{\frac{1}{2}}\right\}\right) ; \\
\tilde{A}_{v, h}^{+}=q_{v, i_{h}}^{-1}\left(\left\{\left(\iota_{h}, z\right) \in \Sigma_{b_{v}, \iota_{h}}:\left|v_{h}\right|_{b}^{\frac{1}{2}} \leq r_{b, h}(z) \leq 2 \delta_{\mathcal{T}}\left(b_{v}\right)\right\}\right) ; \\
S_{v, i}=q_{v, i}^{-1}\left(\left\{(i, z) \in S_{b_{v}, i}: r_{b, h}(z) \geq \delta_{\mathcal{T}}\left(b_{v}\right) \text { if } \iota_{h}=i\right.\right. \\
\left.\left.\left|q_{S}^{-1}(z)\right| \geq \delta_{\mathcal{T}}\left(b_{v}\right) \text { if } i>0\right\}\right) .
\end{gathered}
$$

Let $\tilde{A}_{v, h}$ denote $\tilde{A}_{v, h}^{-} \cup \tilde{A}_{v, h}^{+}$.

Lemma 5.6. For any $p>2$, there exists $C_{p} \in C^{\infty}\left(\mathcal{M}_{\mathcal{T}}^{(0)} ; \mathbb{R}\right)$ such that for any $v \in F^{(0)} \mathcal{T}_{\delta}$ and $h \in \hat{I}$,

$$
\xi \in \tilde{\Gamma}_{c}\left(\tilde{A}_{v, h} ; u_{v}^{*} T V\right) \Longrightarrow\|\xi\|_{C^{0}} \leq C_{p}\left(b_{v}\right)\|\xi\|_{g_{v}, p, 1}
$$

Proof. By construction of the metric $g_{v},\left.g_{v}\right|_{\tilde{A}_{v, h}}$ is the pullback of the metric $g_{v, \iota_{h}}$ on $q_{v, \iota_{h}}\left(\tilde{A}_{v, h}\right)$ by the map $q_{v, \iota_{h}}$. Furthermore, the metric $g_{v, \iota_{h}}$ on $q_{v}\left(\tilde{A}_{v, h}^{ \pm}\right)$differs from the standard metric on the annulus $B_{2 \delta_{\mathcal{T}}\left(b_{v}\right),\left|v_{h}\right|^{\frac{1}{2}}} \subset \mathbb{R}^{2}$ by factors bounded by $C\left(b_{v}\right)$. Since $\left\|d u_{v}\right\|_{g_{v}, p} \leq C_{p}\left(b_{v}\right)$ by (1) of Lemma 3.5, the claim follows from Proposition 3.7 in $[\mathbf{Z 1}]$. 
Proposition 5.7. For any $p>2$, there exists $C_{p} \in C^{\infty}\left(\mathcal{M}_{\mathcal{T}}^{(0)} ; \mathbb{R}\right)$ such that for all $v \in F^{(0)} \mathcal{T}_{\delta}$,

$$
\|\xi\|_{C^{0}} \leq C_{p}\left(b_{v}\right)\|\xi\|_{g_{v}, p, 1} \quad \text { for all } \xi \in \Gamma\left(u_{v}\right) .
$$

Proof. (1) Note that $\left.g_{v}\right|_{S_{v, i}}$ is the pull-back of the metric $g_{b_{v}, i}$ on $q_{v, i}\left(S_{v, i}\right)$ by the map $q_{v, i}$. Thus, by Proposition 3.7 in $[\mathbf{Z 1}]$, if $\xi \in \Gamma_{c}\left(S_{v, i} ; u_{v}^{*} T V\right)$,

$$
\begin{aligned}
\|\xi\|_{C^{0}}=\left\|\xi \circ q_{v, i}\right\|_{C^{0}} & \leq C_{p}\left(\left\|d u_{v} \circ q_{v, i}\right\|_{g_{b_{v}, i}, p}\right)\left\|\xi \circ q_{v, i}\right\|_{g_{b_{v}, i}, p, 1} \\
& =C_{p}\left(b_{v}\right)\|\xi\|_{g_{v}, p, 1},
\end{aligned}
$$

since $\xi$ vanishes outside of $S_{v, i}$.

(2) We now define a partition of unity subordinate to $\left\{S_{v, i}, \tilde{A}_{v, h}: i \in I, h \in\right.$ $\hat{I}\}$. Put

$$
\begin{gathered}
\eta_{v, h}^{+}(z)= \begin{cases}1-\beta_{\delta_{\mathcal{T}}^{2}\left(b_{v}\right)}\left(r_{b_{v}, h}\left(q_{v, \iota_{h}}(z)\right)\right), & \text { if } q_{v, \iota_{h}}(z) \in \Sigma_{b_{v}, \iota_{h}} \\
1, & \text { otherwise; }\end{cases} \\
\eta_{v, h}^{-}(z)= \begin{cases}1-\beta_{\delta_{\mathcal{T}}^{2}\left(b_{v}\right)}\left(\left|q_{S}^{-1} q_{v}(z)\right|\right), & \text { if } q_{v, h}(z) \in \Sigma_{b_{v}, h} \\
1, & \text { otherwise; }\end{cases} \\
\tilde{\eta}_{v}(z)=1-\prod_{h \in \hat{I}} \eta_{v, h}^{-}(z) \eta_{v, h}^{+}(z) .
\end{gathered}
$$

Note that $d \eta_{v, h}^{ \pm}$is supported in $\tilde{A}_{v, h}^{ \pm}$. It follows from the definition of $g_{v}$ that

$$
\left\|d \eta_{v, h}^{ \pm}\right\|_{g_{v}, C^{1}}=\left\|d\left(\eta_{v, h}^{ \pm} \circ q_{v, i_{h}}^{-1}\right)\right\|_{g_{v, i_{h}}, C^{1}} \leq C\left(b_{v}\right) .
$$

Thus, if $\xi \in \Gamma\left(u_{v}\right)$ by (1) and Lemma 5.6,

$$
\begin{aligned}
\|\xi\|_{C^{0}} & \leq \sum_{i \in I}\|\tilde{\eta} \xi\|_{C^{0}\left(S_{v, i}\right)}+\sum_{h \in \hat{I}}\left\|\eta_{v, h}^{-} \eta_{v, h}^{+} \xi\right\|_{C^{0}} \\
& \leq C_{p}\left(b_{v}\right)\left(\|\tilde{\eta} \xi\|_{g_{v}, p, 1}+\sum_{h \in \hat{I}}\left\|\eta_{v, h}^{-} \eta_{v, h}^{+} \xi\right\|_{g_{v}, p, 1}\right) \\
& \leq C_{p}\left(b_{v}\right)\left(|I|\|\xi\|_{g_{v}, p, 1}+2 \sum_{h \in \hat{I}}\left\|\eta_{v, h}^{-} \eta_{v, h}^{+}\right\|_{g_{v}, C^{1}}\|\xi\|_{g_{v}, p}\right) \\
& \leq C_{p}^{\prime}\left(b_{v}\right)\|\xi\|_{g_{v}, p, 1} .
\end{aligned}
$$

5.3. Elliptic Estimates for the Metrics $g_{v}$. This subsection contains the proof of (4) of Lemma 3.5, the main elliptic estimate for the operators $D_{v}$ and the modified Sobolev norms. This estimate does not hold for the standard Sobolev norms. The argument is essentially the same as in $[\mathbf{L T}]$, but we do include all of the details, based on $[\mathbf{Z 1}]$, and state a sharper estimate. 
Let $\mathcal{T}, v, \tilde{A}_{v, h}=\tilde{A}_{v, h}^{-} \cup \tilde{A}_{v, h}^{+}$, and $S_{v, i}$ be as in Subsection 5.2. If $\iota_{h}=\hat{0}$, the metric $g_{b_{v}, \hat{0}}$ is flat on $B_{b_{v}, h}\left(\delta_{\mathcal{T}}\left(b_{v}\right)^{\frac{1}{2}}\right)$. Thus, for any $h \in \hat{I}$, we can choose conformal polar coordinates $(r, \theta)$ on $\tilde{A}_{v, h}$ such that $r(z)=r_{b, v}\left(q_{v, \iota_{h}}(z)\right)$. Since $\left.g_{v}\right|_{\tilde{A}_{v, h}}$ is the pullback of the metric $g_{v, \iota_{h}}$ on $q_{v, \iota_{h}}\left(\tilde{A}_{v, h}\right)$ by the map $q_{v, \iota_{h}}$,

$$
g_{v}=\left\{\left(1-\beta_{\left|v_{h}\right|}(2 r)\right) \frac{2 C\left(b_{v}\right)}{\left|v_{h}\right|+\left|v_{h}\right|^{-1} r^{2}}+\beta_{\left|v_{h}\right|}(r)\right\}\left(d r^{2}+r^{2} d \theta^{2}\right)
$$

on $\tilde{A}_{v, h}$. Similarly, since $\rho_{v}=\rho_{v, \iota_{h}} \circ q_{v, \iota_{h}}$,

$$
\rho_{v}=r^{2}+\frac{\left|v_{h}\right|^{2}}{r^{2}} \quad \text { on } \tilde{A}_{v, h} .
$$

Lemma 5.8. For all $p>1$, there exists $C_{p} \in C^{\infty}\left(\mathcal{M}_{\mathcal{T}}^{(0)} ; \mathbb{R}\right)$ such that for all $v \in F^{(0)} \mathcal{T}_{\delta}, h \in \hat{I}$, and $\xi \in \Gamma_{c}\left(\tilde{A}_{v, h} ; u_{v}^{*} T V\right)$

$$
\left(\int_{\tilde{A}_{v, h}} \rho_{v}^{-\frac{p-2}{p}}\left|\nabla^{b_{v}} \xi\right|^{2}\right)^{\frac{1}{2}} \leq C_{p}\left(b_{v}\right)\left(\left\|D_{v} \xi\right\|_{v, p}+\|\xi\|_{v, p}\right) .
$$

Proof. (1) Let $\epsilon_{1}$ and $\epsilon_{2}$ denote $\left(2 \delta_{\mathcal{T}}\left(b_{v}\right)\right)^{-1}\left|v_{h}\right|$ and $2 \delta_{\mathcal{T}}\left(b_{v}\right)$, respectively. Note that the integral on the left-hand side in the statement of the lemma is conformally invariant. With respect to the metric $d r^{2}+r^{2} d \theta^{2}$,

$$
\left|D_{v} \xi\right|_{(r, \theta)}=\left|\frac{D}{d r} \xi+J r^{-1} \frac{D}{d \theta} \xi\right|_{(r, \theta)},
$$

where $\frac{D}{d r}$ and $\frac{D}{d \theta}$ denote covariant differentiation with respect to the connection $\nabla^{b_{v}}$ and the norms are taken with respect to the metric $g_{V, b}$ on $V$. Thus,

$$
\begin{aligned}
a_{h}^{2} & \equiv \int_{\tilde{A}_{v, h}} \rho_{v}^{-\frac{p-2}{p}}\left|\nabla^{b_{v}} \xi\right|^{2} \\
& \leq\left\|D_{v} \xi\right\|_{v, p}^{2}-2 \int_{0}^{2 \pi} \int_{\epsilon_{1}}^{\epsilon_{2}} \rho_{v}^{-\frac{p-2}{p}}\left\langle\frac{D}{d r} \xi, J \frac{D}{d \theta} \xi\right\rangle d r d \theta .
\end{aligned}
$$

Since $\nabla^{b_{v}} J=0$, using integration by parts twice, we obtain

$$
\begin{aligned}
& \int_{0}^{2 \pi} \int_{\epsilon_{1}}^{\epsilon_{2}} \rho_{v}^{-\frac{p-2}{p}}\left\langle\frac{D}{d r} \xi, J \frac{D}{d \theta} \xi\right\rangle d r d \theta \\
& =-\int_{0}^{2 \pi} \int_{\epsilon_{1}}^{\epsilon_{2}} \rho_{v}^{-\frac{p-2}{p}}\left\langle\frac{D}{d \theta} \frac{D}{d r} \xi, J \xi\right\rangle d r d \theta \\
& =-\int_{0}^{2 \pi} \int_{\epsilon_{1}}^{\epsilon_{2}} \rho_{v}^{-\frac{p-2}{p}}\left(\left\langle\frac{D}{d r} \frac{D}{d \theta} \xi, J \xi\right\rangle-\left\langle\mathcal{R}\left(u_{r}, u_{\theta}\right) \xi, J \xi\right\rangle\right) d r d \theta \\
& =\int_{0}^{2 \pi} \int_{\epsilon_{1}}^{\epsilon_{2}} \rho_{v}^{-\frac{p-2}{p}}\left(\left\langle\frac{D}{d \theta} \xi, J \frac{D}{d r} \xi\right\rangle-\frac{(p-2)}{p} \frac{\rho_{v}^{\prime}(r)}{\rho_{v}(r)}\left\langle\frac{D}{d \theta} \xi, J \xi\right\rangle\right.
\end{aligned}
$$




$$
\left.+\left\langle\mathcal{R}_{b_{v}}\left(u_{r}, u_{\theta}\right) \xi, J \xi\right\rangle\right) d r d \theta
$$

where $u_{r}$ and $u_{\theta}$ denote $\frac{d}{d r} u_{v}$ and $\frac{d}{d \theta} u_{v}$, respectively, and $\mathcal{R}_{b_{v}}$ is the curvature tensor of the connection $\nabla^{b_{v}}$. Since

$$
\left\langle\frac{D}{d \theta} \xi, J \frac{D}{d r} \xi\right\rangle=-\left\langle\frac{D}{d r} \xi, J \frac{D}{d \theta} \xi\right\rangle,
$$

by (5.19) and (1) of Lemma 3.5,

$$
\begin{aligned}
& \left|\int_{0}^{2 \pi} \int_{\epsilon_{1}}^{\epsilon_{2}} \rho_{v}^{-\frac{p-2}{p}}\left\langle\frac{D}{d r} \xi, J \frac{D}{d \theta} \xi\right\rangle d r \theta\right| \\
& \leq \frac{|p-2|}{2 p}\left|\int_{\epsilon_{1}}^{\epsilon_{2}} \rho_{v}^{-\frac{p-2}{p}} \frac{\rho_{v}^{\prime}(r)}{\rho_{v}(r)} \int_{0}^{2 \pi}\left\langle\frac{D}{d \theta} \xi, J \xi\right\rangle d \theta d r\right|+C\left(b_{v}\right)\|\xi\|_{v, p}^{2} .
\end{aligned}
$$

(2) By Poincare Lemma, see Proposition 2.5 in $[\mathbf{Z 1}]$, for every circle with $r$ fixed,

$$
\begin{array}{r}
\left|\int_{0}^{2 \pi}\left\langle\frac{D}{d \theta} \xi, J \xi\right\rangle d \theta\right| \leq \int_{0}^{2 \pi}\left|\frac{D}{d \theta} \xi\right|^{2} d \theta+C\left(g_{b_{v}}\right)\left\{\left(\int_{0}^{2 \pi}\left|u_{\theta}\right|^{2} d \theta\right)\left(\int_{0}^{2 \pi}|\xi|^{2} d \theta\right)\right. \\
\left.+\left(\int_{0}^{2 \pi}\left|u_{\theta}\right| d \theta\right)\left(\int_{0}^{2 \pi}|\xi|^{2} d \theta\right)^{\frac{1}{2}}\left(\int_{0}^{2 \pi}\left|\frac{D}{d \theta} \xi\right|^{2} d \theta\right)^{\frac{1}{2}}\right\} .
\end{array}
$$

Since $\left|\frac{\rho_{v}^{\prime}(r)}{\rho_{v}(r)}\right| \leq 2 r^{-1}$ on $\tilde{A}_{v, h}$, by Holder's inequality and the first part of Lemma 3.5,

$$
\begin{aligned}
& \frac{1}{2} \int_{\epsilon_{1}}^{\epsilon_{2}} \rho_{v}^{-\frac{p-2}{p}}\left|\frac{r \rho_{v}^{\prime}(r)}{\rho_{v}(r)}\right|\left(\int_{0}^{2 \pi} r^{-1}\left|u_{\theta}\right| d \theta\right)\left(\int_{0}^{2 \pi}|\xi|^{2} d \theta\right)^{\frac{1}{2}} \\
& \cdot\left(\int_{0}^{2 \pi} r^{-2}\left|\frac{D}{d \theta} \xi\right|^{2} d \theta\right)^{\frac{1}{2}} r d r \\
& \leq C\|\xi\|_{v, p}\left(\int_{\tilde{A}_{v, h}} \rho_{v}^{-\frac{p-2}{p}} r^{-2}\left|\frac{D}{d \theta} \xi\right|^{2}\right)^{\frac{1}{2}} .
\end{aligned}
$$

Similarly,

$$
\begin{aligned}
& \frac{1}{2} \int_{\epsilon_{1}}^{\epsilon_{2}} \rho_{v}^{-\frac{p-2}{p}}\left|\frac{r \rho_{v}^{\prime}(r)}{\rho_{v}(r)}\right|\left(\int_{0}^{2 \pi} r^{-2}\left|u_{\theta}\right|^{2} d \theta\right)\left(\int_{0}^{2 \pi}|\xi|^{2} d \theta\right) r d r d \theta \\
& \leq C\left(b_{v}\right)\|\xi\|_{v, p}^{2} .
\end{aligned}
$$


Combining equations (5.21)-(5.23), we obtain

$$
\begin{aligned}
& \frac{1}{2}\left|\int_{\epsilon_{1}}^{\epsilon_{2}} \rho_{v}^{-\frac{p-2}{p}} \frac{\rho_{v}^{\prime}(r)}{\rho_{v}(r)} \int_{0}^{2 \pi}\left\langle\frac{D}{d \theta} \xi, J \xi\right\rangle d \theta d r\right| \\
& \leq \int_{0}^{2 \pi} \int_{\epsilon_{1}}^{\epsilon_{2}} \rho_{v}^{-\frac{p-2}{p}} r^{-2}\left|\frac{D}{d \theta} \xi\right|^{2} r d r d \theta+C\left(b_{v}\right)\left(\|\xi\|_{v, p}^{2}+\|\xi\|_{p} a_{h}\right) .
\end{aligned}
$$

Note that

$$
\begin{aligned}
& \int_{0}^{2 \pi} \int_{\epsilon_{1}}^{\epsilon_{2}} \rho_{v}^{-\frac{p-2}{p}} r^{-2}\left|\frac{D}{d \theta} \xi\right|^{2} r d r d \theta \\
& =\frac{1}{2} \int_{\tilde{A}_{v, h}} \rho_{v}^{-\frac{p-2}{p}}\left(r^{-2}\left|\frac{D}{d \theta} \xi\right|^{2}+\left|\left(\frac{D}{d r} \xi+J r^{-1} \frac{D}{d \theta} \xi\right)-\frac{D}{d r} \xi\right|^{2}\right) \\
& \leq \frac{1+\epsilon}{2} a_{h}^{2}+C_{\epsilon}\left\|D_{v} \xi\right\|_{v, p}^{2}
\end{aligned}
$$

for any $\epsilon>0$. Combining equations (5.18), (5.20), (5.24) and (5.25), we obtain

$$
a_{h}^{2} \leq \frac{|p-2|}{p}(1+\epsilon) a_{h}^{2}+\left(C\left(b_{v}\right)+C_{\epsilon}\right)\left(\left\|D_{v} \xi\right\|_{v, p}^{2}+\|\xi\|_{v, p}^{2}+\|\xi\|_{v, p} a_{h}\right) .
$$

Since $\frac{|p-2|}{p}<1$, the claim follows by choosing $\epsilon$ sufficiently small.

Lemma 5.9. For all $p \geq 1$, there exists $C_{p} \in C^{\infty}\left(\mathcal{M}_{\mathcal{T}}^{(0)} ; \mathbb{R}\right)$ such that for all $v \in F^{(0)} \mathcal{T}_{\delta}, h \in \hat{I}$, and $\xi \in \Gamma_{c}\left(\tilde{A}_{v, h} \mid u_{v}^{*} T V\right)$

$$
\left\|\nabla^{b_{v}} \xi\right\|_{g_{v}, p} \leq C_{p}\left(b_{v}\right)\left(\left\|D_{v} \xi\right\|_{v, p}+\|\xi\|_{v, p}+\left(\int_{\tilde{A}_{v, h}} \rho_{v}^{-\frac{p-2}{2 p}}|\nabla \xi|^{2}\right)^{\frac{1}{2}}\right) .
$$

Proof. Choose a sequence

$$
\delta_{0}>\ldots>\delta_{N+1}>0 \quad \text { such that } \delta_{0}=\epsilon_{2}, \delta_{N+1}=\epsilon_{1}, \frac{1}{3} \leq \frac{\delta_{l+1}}{\delta_{l}} \leq \frac{1}{2} .
$$

For each $l=1, \ldots, N-1$, let $g_{l}$ denote the metric

$$
g_{l}=\left(\delta_{l}^{2}+\frac{\left|v_{h}\right|^{2}}{\delta_{l}^{2}}\right)^{-1} g_{v} \text { on } \tilde{A}_{l}=\left\{(r, \theta) \in \tilde{A}_{v, h}: \delta_{l+2} \leq r \leq \delta_{l-1}\right\} .
$$

Let $\rho_{l}=\delta_{l}^{2}+\left|v_{h}\right|^{2} \delta_{l}^{-2}$ and denote by $A_{l}$ the annulus

$$
\left\{(r, \theta) \in \tilde{A}_{v, h}: \delta_{l+1} \leq r \leq \delta_{l}\right\} .
$$

The pullback of the metric $g_{l}$ on $\tilde{A}_{l}$ to the annulus

$$
\left(\frac{\delta_{l+2}}{\delta_{l}}, \frac{\delta_{l-1}}{\delta_{l}}\right) \times S^{1} \subset \mathbb{R}^{2}
$$


by the map $(r, \theta) \longrightarrow\left(\delta_{l} r, \theta\right)$ differs from the Eucledian metric by a conformal factor bounded by $C\left(b_{v}\right)$, since

$$
\begin{aligned}
\frac{1}{100} & \leq\left\{\left(1-\beta_{\left|v_{h}\right|}\left(\delta_{l} r\right)\right) \frac{2}{\left|v_{h}\right|+\left|v_{h}\right|^{-1} \delta_{l}^{2} r^{2}}+\beta_{\left|v_{h}\right|}\left(\delta_{l} r\right)\right\}\left(\delta_{l}^{2}+\frac{\left|v_{h}\right|^{2}}{\delta_{l}^{2}}\right)^{-\frac{1}{2}} \delta_{l} \\
& \leq 200
\end{aligned}
$$

whenever $r \in\left(\frac{1}{9}, 3\right)$ and $\delta_{l} \in\left(\left|v_{h}\right|, 1\right)$. Thus, by Proposition 3.10 in $[\mathbf{Z 1}]$,

$$
\begin{aligned}
\left\|\nabla^{b_{v}} \xi\right\|_{g_{l}, L^{p}\left(A_{l}\right)} \leq C\left(\left\|D_{v} \xi\right\|_{g_{l}, L^{p}\left(\tilde{A}_{l}\right)}+\right. & \left\|\nabla^{b_{v}} \xi\right\|_{g_{l}, L^{p}\left(\tilde{A}_{l}\right)} \\
& \left.+\|\xi d u\|_{g_{l}, L^{p}\left(\tilde{A}_{l}\right)}\right),
\end{aligned}
$$

or equivalently

$$
\begin{array}{r}
\left\|\nabla^{b_{v}} \xi\right\|_{g_{v}, L^{p}\left(A_{l}\right)} \leq C\left(\left\|D_{v} \xi\right\|_{g_{v}, L^{p}\left(\tilde{A}_{l}\right)}+\left\|\rho_{l}^{-\frac{p-2}{2 p}} \nabla^{b_{v}} \xi\right\|_{L^{2}\left(\tilde{A}_{l}\right)}\right. \\
\left.+\|\xi d u\|_{g_{v}, L^{p}\left(\tilde{A}_{l}\right)}\right) .
\end{array}
$$

Since $\frac{\rho_{v}(r)}{\rho_{l}} \in\left[\frac{1}{81}, 81\right]$ when $r \in\left[\delta_{l+2}, \delta_{l-1}\right],(5.27)$ is equivalent to

$$
\begin{array}{r}
\left(\int_{A_{l}}\left|\nabla^{b_{v}} \xi\right|^{p}\right)^{\frac{1}{p}} \leq C_{p}\left(b_{v}\right)\left(\left(\int_{\tilde{A}_{l}}\left|D_{v} \xi\right|^{p}\right)^{\frac{1}{p}}+\left(\int_{\tilde{A}_{l}} \rho_{v}^{-\frac{p-2}{p}}\left|\nabla^{b_{v}} \xi\right|^{2}\right)^{\frac{1}{2}}\right. \\
\left.+\left\|\xi d u_{v}\right\|_{g_{v}, L^{p}\left(\tilde{A}_{l}\right)}\right) .
\end{array}
$$

The claim follows by summing up the last inequality over all $l$ and using (1) of Lemma 3.5.

Remark. The above proof does not quite apply to the two outermost annuli $A_{1}$ and $A_{N}$. However, since $\xi$ is compactly supported in $\tilde{A}_{v, h}$, the proof of Proposition 3.10 in $[\mathbf{Z 1}]$ can be applied to $A_{1}$ with $A_{1} \cup A_{2}$ replacing $\tilde{A}_{1}$ to (5.26), and similarly for $A_{N}$. Alternatively, for the purposes of proving Proposition 5.11 below, it is sufficient to prove Lemma 5.9 and Corollary 5.10 for $\xi$ that vanish on $A_{1}$ and $A_{N}$.

Corollary 5.10. For all $p>1$, there exists $C_{p} \in C^{\infty}\left(\mathcal{M}_{\mathcal{T}}^{(0)} ; \mathbb{R}\right)$ such that for all $v \in F^{(0)} \mathcal{T}_{\delta}, h \in \hat{I}$, and $\xi \in \Gamma_{c}\left(\tilde{A}_{v, h} ; u^{*} T V\right)$

$$
\|\xi\|_{v, p, 1} \leq C_{p}\left(b_{v}\right)\left(\left\|D_{v} \xi\right\|_{v, p}+\|\xi\|_{v, p}\right) .
$$

Proof. This corollary follows immediately from Lemmas 5.10 and 5.10.

Proposition 5.11. For all $p>1$, there exists $C_{p} \in C^{\infty}\left(\mathcal{M}_{\mathcal{T}}^{(0)} ; \mathbb{R}\right)$ such that for all $v \in F^{(0)} \mathcal{T}_{\delta}$,

$$
\|\xi\|_{v, p, 1} \leq C_{p}\left(b_{v}\right)\left(\left\|D_{v} \xi\right\|_{v, p}+\|\xi\|_{v, p}\right) \quad \forall \xi \in \Gamma\left(u_{v}\right) .
$$


Proof. This proposition follows from Corollary 5.10 and Proposition 3.12 in $[\mathbf{Z 1}]$ by taking a partition of unity as in the proof of Proposition 5.7.

\subsection{Fiber-Uniform Inverse for the Operator $D_{v}$.}

Lemma 5.12. Let $\left\{v_{k}\right\}$ be a sequence in $F^{(0)} \mathcal{T}_{\delta}$ that converges to $b^{*} \in \mathcal{M}_{\mathcal{T}}^{(0)}$. Suppose $\xi_{k} \in \Gamma\left(u_{v_{k}}\right)$ is such that $\left\|\xi_{k}\right\|_{v_{k}, p, 1} \leq 1$ for all $k$, while $\left\|D_{v_{k}} \xi_{k}\right\|_{v_{k}, p}$ tends to 0 as $k \longrightarrow \infty$ for some $p>2$. Then a subsequence of $\left\{\xi_{k}\right\} C^{0}$ converges $\xi^{*} \in \Gamma_{-}\left(b^{*}\right)$. Furthermore, $\left\|\xi_{k}\right\|_{v_{k}, C^{0}}$ converges to $\left\|\xi^{*}\right\|_{b^{*}, C^{0}}$.

Proof. (1) Write $b^{*}=\left(S, M, I ; x^{*},\left(j, y^{*}\right), u\right)$ and

$$
v_{k}=\left(b_{k}, v_{k}\right)=\left(\left(S, M, I ; x_{k},\left(j, y_{k}\right), u_{k}\right),\left(v_{k}\right)_{\hat{I}}\right) .
$$

For each $i \in I$ and $\delta>0$, put

$$
S_{i, \delta}^{*}=\left\{z \in \Sigma_{b, i}: r_{b^{*}, h}(z) \geq \delta \forall h \in \hat{I} ;\left|q_{S}^{-1}(z)\right| \geq \delta \text { if } i>0\right\} .
$$

For $i \in I$ and all $k$ sufficiently large (depending on $\delta$ ), define $\zeta_{k, i}, \xi_{k, i}^{\prime}$ in $\Gamma\left(\left.u_{i}^{*}\right|_{S_{i, \delta}^{*}}\right)$ by

$$
\begin{gathered}
\exp _{b^{*}, u_{i}^{*}(z)} \zeta_{k, i}(z)=u_{v_{k}}\left(q_{v_{k}}^{-1}(z)\right),\left\|\zeta_{k, i}\right\|_{b^{*}, C^{0}}<\operatorname{inj} g_{V, b^{*}} \\
\Pi_{b^{*}, \zeta_{k, i}(z)} \xi_{k, i}^{\prime}(z)=\xi_{k}\left(q_{v_{k}}^{-1}(z)\right) .
\end{gathered}
$$

Since $\left\|\nabla^{b^{*}} \zeta_{k, i}\right\|_{b^{*}, C^{0}} \leq C$ for $k$ sufficiently large, (1) of Lemma 3.5 and by Corollary 2.3 in $[\mathbf{Z} 1]$,

$$
\begin{aligned}
& \left\|\xi_{k, i}^{\prime}\right\|_{b^{*}, p, 1} \leq\left(1+\epsilon_{k}\right)\left\|\xi_{k}\right\|_{v_{k}, p, 1}+\epsilon_{k}\left\|\xi_{k}\right\|_{v_{k}, C^{0}}, \\
& \left\|D_{b, u_{i}} \xi_{k, i}^{\prime}\right\|_{b^{*}, p} \leq\left(1+\epsilon_{k}\right)\left\|D_{v_{k}} \xi_{k}\right\|_{v_{k}, p}+\epsilon_{k}\left\|\xi_{k}\right\|_{v_{k}, C^{0}},
\end{aligned}
$$

where $\epsilon_{k} \longrightarrow 0$ as $k \longrightarrow \infty$. Since $\left\|\xi_{k}\right\|_{v_{k}, p, 1} \leq 1$, (2) of Lemma 3.5 applied to $(5.28)$,

$$
\begin{aligned}
& \left\|\xi_{k, i}^{\prime}\right\|_{b^{*}, p, 1} \leq\left(1+\tilde{\epsilon}_{k}\right)\left\|\xi_{k}\right\|_{v_{k}, p, 1}+\tilde{\epsilon}_{k}, \\
& \left\|D_{b, u_{i}} \xi_{k, i}^{\prime}\right\|_{b^{*}, p} \leq\left(1+\tilde{\epsilon}_{k}\right)\left\|D_{v_{k}} \xi_{k}\right\|_{v_{k}, p}+\tilde{\epsilon}_{k},
\end{aligned}
$$

where $\tilde{\epsilon}_{k} \longrightarrow 0$ as $k \longrightarrow \infty$. Sobolev's embedding theorem then implies that $\xi_{k, i}^{\prime}$ converges to a vector field $\xi_{i}^{*} \in \Gamma\left(\left.u_{i}\right|_{\Sigma_{b^{*}, i}^{*}}\right)$ in the $C^{0}$-norm on the compact subsets of $\Sigma_{b^{*}, i}^{*}$. Furthermore, $\left\|\xi_{i}^{*}\right\|_{b^{*}, C^{0}}<\infty$, since

$$
\left\|\xi_{k, i}^{\prime}\right\|_{b^{*}, C^{0}} \leq\left(1+\epsilon_{k}\right)\left\|\xi_{k}\right\|_{v_{k}, C^{0}} \leq C .
$$

(2) We will now show that $D_{b^{*}, u_{i}^{*}} \xi_{i}^{*}=0$ weakly, i.e., $\left\langle\left\langle\xi_{i}^{*}, D_{b^{*}, u_{i}^{*}}^{*} \eta\right\rangle\right\rangle_{b^{*}}=0$ for any $\eta \in \Gamma^{0,1}\left(u_{i}^{*}\right)$. We have

$$
\begin{aligned}
\left\langle\left\langle\xi_{i}^{*}, D_{b^{*}, u_{i}^{*}}^{*} \eta\right\rangle\right\rangle_{b^{*}} & =\lim _{\delta \longrightarrow 0} \int_{S_{i, \delta}^{*}}\left\langle\xi_{i}^{*}, D_{b^{*}, u_{i}^{*}}^{*} \eta\right\rangle_{b^{*}} \\
& =\lim _{\delta \longrightarrow 0} \lim _{k \longrightarrow \infty} \int_{S_{i, \delta}^{*}}\left\langle\xi_{k, i}^{\prime}, D_{b^{*}, u_{i}^{*}}^{*} \eta\right\rangle_{b^{*}},
\end{aligned}
$$


since $\xi_{k, i}^{\prime} \longrightarrow \xi_{i}^{*}$ in the $C^{0}$-norm on $S_{i, \delta}$. By integration by parts,

$$
\begin{aligned}
\left|\int_{S_{i, \delta}^{*}}\left\langle\xi_{k, i}^{\prime}, D_{b^{*}, u_{i}^{*}}^{*} \eta\right\rangle_{b^{*}}-\int_{S_{i, \delta}^{*}}\left\langle D_{b^{*}, u_{i}^{*}} \xi_{k, i}^{\prime}, \eta\right\rangle_{b^{*}}\right| & \leq C \int_{\partial S_{i, \delta}^{*}}\left|\xi_{k, i}^{\prime} \| \eta\right| \\
& \leq C^{\prime}\left\|\xi_{k, i}^{\prime}\right\|_{b^{*}, C^{0}}\|\eta\|_{b^{*}, C^{0}} \delta
\end{aligned}
$$

Since $\left\|D_{b^{*}, u_{i} *} \xi_{k, i}^{\prime}\right\|_{b^{*}, p} \longrightarrow 0$ as $k \longrightarrow \infty$ on $S_{i, \delta}^{*}$ and $\left\|\xi_{k, i}^{\prime}\right\|_{b^{*}, C^{0}} \leq C$, by (5.30) and (5.31),

$$
\left\langle\left\langle\xi_{i}^{*}, D_{b^{*}, u_{i}^{*}}^{*} \eta\right\rangle\right\rangle_{b^{*}}=0 \quad \forall \eta \in \Gamma^{0,1}\left(u_{i}^{*}\right) .
$$

(3) Since $D_{b^{*}, u_{i}^{*}} \xi_{i}^{*}=0$ weakly on $S_{i}$ and $D_{b^{*}, u_{i}^{*}}$ is an elliptic operator, it follows that $\xi_{i}^{*}$ is smooth and $D_{b^{*}, u_{i}^{*}} \xi_{i}^{*}=0$. It will now be shown that $\xi_{\iota_{h}}^{*}\left(x_{h}^{*}\right)=\xi_{h}^{*}(\infty)$ for all $h \in \hat{I}$, i.e., $\xi^{*} \equiv \xi_{I}^{*} \in \Gamma\left(b^{*}\right)$. For each $h \in \hat{I}$, let $A_{h, \delta, k} \subset S$ denote the small cylinder connecting $q_{v_{k}}^{-1}\left(S_{h, \delta}^{*}\right)$ and $q_{v_{k}}^{-1}\left(S_{\iota_{h}, \delta}^{*}\right)$. Let $\epsilon>0$ be any small number. Choose small $\delta>0$ such that $u_{h}\left(B_{b^{*}, h}(\infty, 2 \delta)\right)$ and $u_{\iota_{h}}^{*}\left(B_{b^{*}, \iota_{h}^{*}}\left(x_{h}^{*}, 2 \delta\right)\right)$ lie in $B_{b^{*}}\left(u_{h}^{*}(\infty), \epsilon\right)$. Then we can write

$$
u_{b^{*}}^{*}(z)=\exp _{b^{*}, u_{b^{*}}^{*}\left(x_{h}^{*}\right)} \bar{u}_{b^{*}}(z),\left|\bar{u}_{b^{*}}(z)\right|<\operatorname{inj} g_{V, b^{*}} ; \quad \bar{\xi}_{k}^{\prime}(z) \equiv \Pi_{b^{*}, \bar{u}_{b}(z)}^{-1} \xi_{k}^{\prime}(z)
$$

for $z \in B_{b^{*}, h}(\infty, \delta) \cup B_{b^{*}, \iota_{h}^{*}}\left(x_{h}^{*}, \delta\right)$. Similarly, put

$$
\bar{\xi}_{h}^{*}(z)=\Pi_{b^{*}, \bar{u}_{b^{*}}(z)}^{-1} \xi_{h}^{*}(z) \text { and } \bar{\xi}_{\iota_{h}}^{*}(z)=\Pi_{b^{*}, \bar{u}_{b^{*}}(z)}^{-1} \xi_{\iota_{h}}^{*}(z)
$$

for $z$ in $B_{b^{*}, h}(\infty, \delta)$ and in $B_{b^{*}, i_{h}}\left(x_{h}^{*}, \delta\right)$, respectively. We can also assume that $\delta$ is so small that $\left|\bar{\xi}_{h}^{*}-\xi_{h}^{*}(\infty)\right|_{b^{*}}$ and $\left|\bar{\xi}_{\iota_{h}}^{*}-\xi_{i_{h}}^{*}\left(x_{h}^{*}\right)\right|_{b^{*}}$ do not exceed $\epsilon$ on $B_{b^{*}, h}(\infty, \delta)$ and on $B_{b^{*}, \iota_{h}}\left(x_{h}^{*}, \delta\right)$, respectively. Choose large $k^{*}$ such that all $k>k^{*}$

$$
\left\|\xi^{*}-\xi_{k}^{\prime}\right\|_{C^{0}\left(S_{h, \delta}^{*} \cup S_{\iota_{h}, \delta}^{*}\right)} \leq \epsilon .
$$

It can be assumed that $u_{k}\left(A_{h, 2 \delta, k}\right)$ lies in $B_{b^{*}}\left(u^{*}\left(x_{h}^{*}\right) ; 2 \epsilon\right)$ for $k>k^{*}$. Thus, we can write

$$
u_{k}(z)=\exp _{b^{*}, u\left(x_{h}^{*}\right)} \bar{u}_{k}(z),\left|\bar{u}_{k}(z)\right|_{b^{*}}<\operatorname{inj} g_{V, b^{*}} ; \quad \bar{\xi}_{k}(z) \equiv \Pi_{b^{*}, \bar{u}_{k}(z)}^{-1} \xi_{k}(z)
$$

if $z \in A_{h, \delta, k}$. Pick points $z_{1}$ and $z_{2}$, one on each component of the boundary of $A_{h, \delta, k}$. Then

$$
\begin{aligned}
\left|\xi_{h}^{*}(\infty)-\xi_{\iota_{h}}^{*}\left(x_{h}^{*}\right)\right|_{b^{*}} \leq & 2\left(\epsilon+\left|\bar{\xi}_{h}^{*}\left(q_{v_{k}}\left(z_{1}\right)\right)-\bar{\xi}_{\iota_{h}}^{*}\left(q_{v_{k}}\left(z_{2}\right)\right)\right|_{b^{*}}\right) \\
\leq & 4\left(\epsilon+\left|\bar{\xi}_{k, h}^{\prime}\left(q_{v_{k}}\left(z_{1}\right)\right)-\bar{\xi}_{k, i_{h}}^{\prime}\left(q_{v_{k}}\left(z_{2}\right)\right)\right|_{b^{*}}\right) \\
\leq & C\left(\epsilon+\left|\bar{\xi}_{k}\left(z_{1}\right)-\bar{\xi}_{k}\left(z_{2}\right)\right|_{b^{*}}\right. \\
& \left.+\left\|\zeta_{k}\right\|_{b^{*}, C^{0}\left(S_{h, \delta}^{*} \cup S_{i_{h}, \delta}^{*}\right)}\left\|\bar{\xi}_{k}\right\|_{b^{*}, C^{0}\left(A_{h, \delta, k}\right)}\right) .
\end{aligned}
$$

Since $A_{h, \delta, k}$ is uniformly equivalent to the union of two annuli with the larger radius bounded above by $\delta$ and the smaller radius less than half of the larger, 
by Lemma 3.1 in $[\mathbf{Z 1}]$ and Holder's inequality,

$$
\begin{aligned}
\left|\bar{\xi}_{k}\left(z_{1}\right)-\bar{\xi}_{k}\left(z_{2}\right)\right|_{b^{*}} & \leq C\left|\bar{\xi}_{k}\left(z_{1}\right)-\bar{\xi}_{k}\left(z_{2}\right)\right|_{b_{k}} \\
& \leq C^{\prime} \delta^{\frac{2(p-2)}{p}}\left\|d \bar{\xi}_{k}\right\|_{v_{k}, L^{p}\left(A_{h, \delta, k}\right)} .
\end{aligned}
$$

By Corollary 2.3 in $[\mathbf{Z 1}]$ and Proposition 5.11,

$$
\left\|d \bar{\xi}_{k}\right\|_{v_{k}, L^{p}\left(A_{h, \delta, k}\right)} \leq\left\|\xi_{k}\right\|_{v_{k}, p, 1}+\left\|d u_{v_{k}}\right\|_{v_{k}, p}\left\|\xi_{k}\right\|_{v_{k}, C^{0}} \leq C .
$$

Combining equations (5.32)-(5.34), we obtain

$$
\left|\xi_{h}^{*}(\infty)-\xi_{\iota_{h}}^{*}\left(x_{h}^{*}\right)\right|_{b^{*}} \leq C\left(\epsilon+\delta^{\frac{2(p-2)}{p}}+\left\|\zeta_{k}\right\|_{b^{*}, C^{0}\left(S_{h, \delta}^{*} \cup S_{\iota_{h}, \delta}^{*}\right)}\right) .
$$

Since the last term in (5.35) tends to zero as $k \longrightarrow \infty$ and $\epsilon$ and $\delta$ can be chosen to be arbitrarily small, it follows $\xi_{h}^{*}(\infty)=\xi_{\iota_{h}}^{*}\left(x_{h}^{*}\right)$.

Proposition 5.13. For any simple bubble type $\mathcal{T}$, there exist $\mathbb{R}^{+}$-valued smooth functions $C, \delta$ on $\mathcal{M}_{\mathcal{T}}^{(0)}$ such that for all $v \in F^{(0)} \mathcal{T}_{\delta}$ if $\mathcal{T}$ is regular and $v \in F^{(\emptyset)} \mathcal{T}_{\delta}$ if $\mathcal{T}$ is semiregular,

$$
\|\xi\|_{v, p, 1} \leq C_{p}\left(b_{v}\right)\left\|D_{v} \xi\right\|_{v, p} \quad \forall \xi \in \Gamma_{+}(v) \quad \text { and } \quad \forall \xi \in \tilde{\Gamma}_{+}(v) .
$$

Proof. If not, we can choose a sequence $v_{k} \in F^{(0)} \mathcal{T}_{\delta}$, converging to some $b \in \mathcal{M}_{\mathcal{T}}^{(0)}$ and vector fields $\xi_{k} \in \Gamma_{+}\left(v_{k}\right)$ (or $\left.\xi_{k} \in \tilde{\Gamma}_{+}\left(v_{k}\right)\right)$ such that

$$
\left\|\xi_{k}\right\|_{v_{k}, p, 1}=1 \quad \text { and } \quad \lim _{k \longrightarrow \infty}\left\|D_{v_{k}} \xi\right\|_{v_{k}, p}=0 .
$$

If $\xi_{k} \in \Gamma_{+}\left(v_{k}\right)$, note that $\left\{\Gamma_{-}\left(v_{k}\right)\right\} C^{0}$-converges to $V \equiv \Gamma_{-}(b)$. If $\xi_{k} \in \tilde{\Gamma}_{+}\left(v_{k}\right)$, by Definition 3.11, a subsequence of $\left\{\tilde{\Gamma}_{-}\left(v_{k}\right)\right\} C^{0}$-converges to a subspace $V \subset L_{1}^{p}(b)$ such that $\pi_{b,-}: V \longrightarrow \Gamma_{-}(b)$ is an isomorphism. In either case, by the first statement of Lemma 5.12, a subsequence of $\left\{\xi_{k}\right\} C^{0}$-converges to a vector field $\xi^{*} \in \Gamma_{-}(b)$. By the second statement of Lemma 5.12, $\xi^{*}$ must be orthogonal $V$, since $\xi_{k} \in \Gamma_{+}\left(v_{k}\right)$ (or $\xi_{k} \in \tilde{\Gamma}_{+}\left(v_{k}\right)$ ). Thus, $\xi^{*}=0$. On the other hand, by Proposition 5.11, there exists $\epsilon>0$ such that $\left\|\xi_{k}\right\|_{v_{k}, p} \geq \epsilon$ for all $k$ sufficiently large. However, by Lemma $5.12,\left\|\xi_{k}\right\|_{v_{k}, C^{0}} \longrightarrow 0$, which is a contradiction.

\section{References}

[FO] K. Fukaya and K. Ono, Arnold Conjecture and Gromov-Witten Invariants, Topology 38(5) (1999), 933-1048.

[GH] P. Griffiths and J. Harris, Principles of Algebraic Geometry, 1994.

[I] E. Ionel, Genus-One Enumerative Invariants in $\mathbb{P}^{n}$ with Fixed $j$-Invariant, Duke Math. J. 94(2) (1998), 279-324.

[LM] H. Lawson and M. Michelsohn, Spin Geometry, Princeton University Press, 1989. 
[LT] J. Li and G. Tian, Virtual moduli cycles and Gromov-Witten invariants of general symplectic manifolds, Topics in symplectic 4-manifolds, 47-83, First Int. Press Lect. Ser., I, Internat. Press, 1998.

[MS] D. McDuff and D. Salamon, J-holomorphic Curves and Quantum Cohomology, American Mathematical Society, 1994.

[RT] Y. Ruan and G. Tian, A Mathematical Theory of Quantum Cohomology, J. Differential Geom. 42(2) (1995), 259-367.

[T] C. Taubes, Self-dual Connections on 4-Manifolds with Indefinite Intersection Matrix, J. Differential Geom. 19 (1984), 517-560.

[Z1] A. Zinger, Basic Estimates of Riemannian Geometry Used in Gluing Pseudoholomorphic Maps, notes.

[Z2] _ Enumeration of Genus-Two Curves with a Fixed Complex Structure in $\mathbb{P}^{2}$ and $\mathbb{P}^{3}$, J. Differential Geom. 65(3) (2003), 341-467.

[Z3] , Counting Rational Curves of Arbitrary Shape in Projective Spaces, math. AG/0210146.

[Z4] On a Compactification of the Moduli Space of Holomorphic Maps from Smooth Genus-One Riemann Surfaces, math.SG/0406103.

Received 01/18/2002, accepted 12/23/2004. Partially supported by NSF Graduate Research Fellowship and NSF grant DMS-9803166.

Department of Mathematics

MIt, 77 Massachusetts Ave.

Cambridge, MA 02139

Current address:

Department of Mathematics

Stanford University

Stanford, CA 94305-2125

E-mail address: azinger@math.stanford.edu 